\title{
Reversible Multicomponent AND Gate Triggered by Stoichiometric Chemical Pulses Commands the Self-Assembly and Actuation of Catalytic Machinery
}

\author{
Pronay Kumar Biswas, Suchismita Saha, Sudhakar Gaikwad and Michael Schmittel* \\ Center of Micro and Nanochemistry and Engineering, Organische Chemie I, \\ Universität Siegen, Adolf-Reichwein-Str. 2, D-57068 Siegen, Germany \\ E-mail: schmittel@chemie.uni-siegen.de
}

Table of Contents:

\begin{tabular}{|l|l|}
\hline 1. Synthesis & $\mathrm{S} 2$ \\
\hline 1.1 General information & $\mathrm{S} 2$ \\
\hline 1.2 Synthesis and characterization of ligands and complexes & $\mathrm{S} 3-\mathrm{S} 28$ \\
\hline 2. NMR Spectra: ${ }^{1} \mathrm{H},{ }^{13} \mathrm{C},{ }^{1} \mathrm{H}-{ }^{-1} \mathrm{H}$ COSY & $\mathrm{S} 29-\mathrm{S} 52$ \\
\hline 3. DOSY NMR Spectra & $\mathrm{S} 53$ \\
\hline 4. VT ${ }^{1} \mathrm{H}-\mathrm{NMR}$ Spectra & $\mathrm{S} 53$ \\
\hline 5. NMR Simulation Analysis to Determine the Rotational Frequency & $\mathrm{S} 54$ \\
\hline 6. Catalytic Experiments & $\mathrm{S} 55-\mathrm{S} 64$ \\
\hline 7. ESI-MS Spectra & $\mathrm{S} 65-\mathrm{S} 78$ \\
\hline 8. Speciation Distribution Analysis & $\mathrm{S} 79$ \\
\hline 9. References & $\mathrm{S} 80$ \\
\hline
\end{tabular}




\section{Synthesis}

\subsection{General information}

All reagents were obtained from commercial suppliers and used without further purification. Technical grade solvents were distilled prior to use. Tetrahydrofuran (THF) was predried over basic alumina and then distilled over potassium. Dimethylformamide (DMF) and triethylamine $\left(\mathrm{Et}_{3} \mathrm{~N}\right)$ were distilled over calcium hydride. Diethyl ether $\left(\mathrm{Et}_{2} \mathrm{O}\right)$ was predried over calcium hydride and then distilled from sodium. Melting points of compounds were measured using a Büchi SMP-11 instrument. ${ }^{1} \mathrm{H},{ }^{13} \mathrm{C}$, and ${ }^{1} \mathrm{H}^{-1} \mathrm{H}$ COSY NMR spectra were recorded on a Bruker Avance 400 and Jeol ECZ 500 at 298 K. DOSY NMR was recorded on a Varian VNMR-S600 MHz. Chemical shifts refer to the residual protiated fraction of the NMR solvent $\left(\mathrm{CDCl}_{3}: \delta_{\mathrm{H}}=\right.$ $\left.7.26 \mathrm{ppm}, \delta_{\mathrm{C}}=77.0 \mathrm{ppm} ; \mathrm{CD}_{2} \mathrm{Cl}_{2}: \delta_{\mathrm{H}}=5.32 \mathrm{ppm}, \delta_{\mathrm{C}}=53.8 \mathrm{ppm}\right)$. Abbreviations in ${ }^{1} \mathrm{H} \mathrm{NMR}$ assignments are used to describe splitting patterns ( $\mathrm{s}$ : singlet, $\mathrm{d}$ : doublet, $\mathrm{t}$ : triplet, dd: doublet of doublets, ddd: doublet of doublets of doublets, bs: broad singlet, td: triplet of doublets, m: multiplet), the value of coupling constant(s) is reported in $\mathrm{Hertz}(\mathrm{Hz})$ and the number of protons are implied. Numbering of the carbon atoms is not in accordance with IUPAC nomenclature. UV-vis spectra were measured on Cary Win 50. Electrospray ionization-mass spectra (ESIMS) were recorded on a Thermo-Quest LCQ Deca instrument. Infrared spectra were recorded using a Perkin Elmer Spectrum-Two FT-IR spectrometer. Column chromatography was performed on silica gel 60 (60-230 mesh) or on neutral alumina (0.05-0.15 mm, Brockmann Activity 1). Thin layer chromatography (TLC) was performed using Merck silica gel (60 F254) or on neutral $\mathrm{Al}_{2} \mathrm{O}_{3}(150 \mathrm{~F} 254)$ sheets. Size exclusion chromatography was performed on BioRad Biobeads-SX3 using toluene or THF as an eluent. 


\subsection{Synthesis and characterization of ligands and complexes}

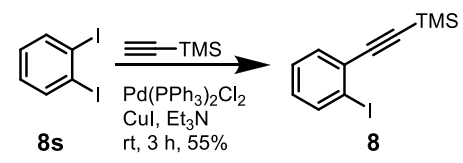

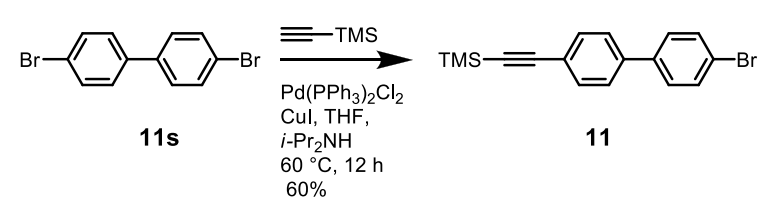

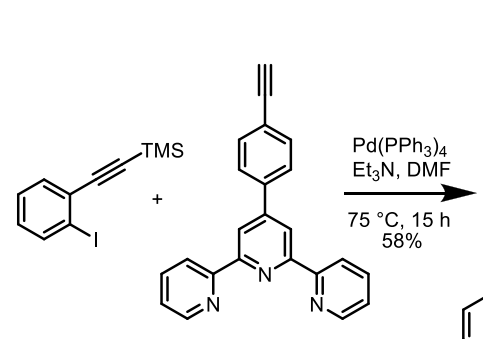

8

9s<smiles></smiles>

9
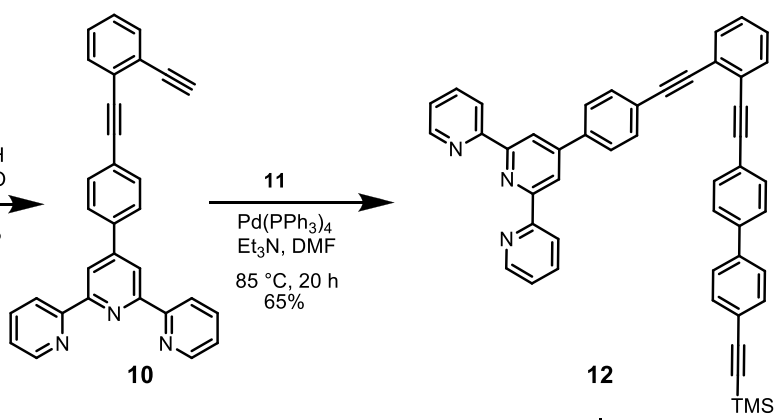

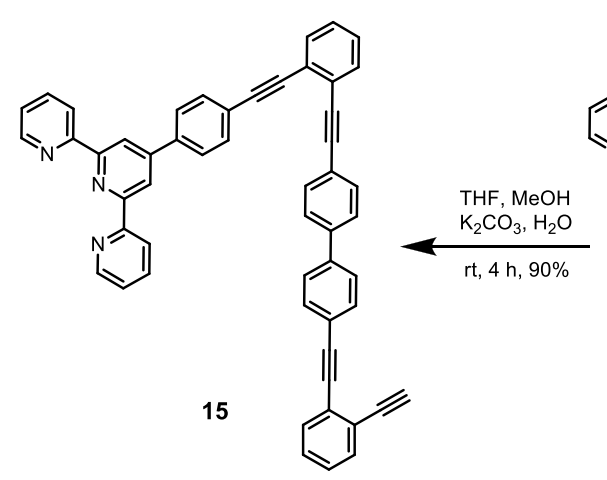

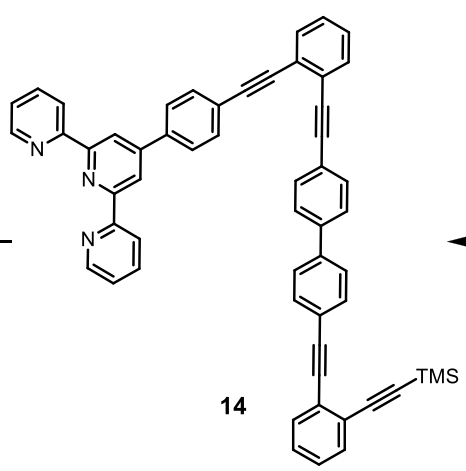

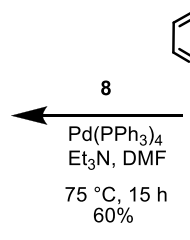
$\mathrm{rt}, 4 \mathrm{~h}, 90 \%\left[\begin{array}{l}\mathrm{THF}, \mathrm{MeOH} \\ \mathrm{K}_{2} \mathrm{CO}_{3}, \mathrm{H}_{2} \mathrm{O}\end{array}\right.$ ${ }_{60 \%}^{\circ} \mathrm{C}, 15 \mathrm{~h}$<smiles>C=C(C)c1ccc(-c2ccc(C=Cc3ccccc3C=Cc3ccc(-c4cc(-c5ccc[nH]5)nc(-c5ccc[nH]5)c4)cc3)cc2)cc1</smiles><smiles>C#Cc1cc2ccc3ccc(-c4c(OC)cc(OC)cc4OC)nc3nc-2c1C(C)=C(C)C</smiles>

$16 s$<smiles>CCCCOc1cc(I)c(OCCCC)cc1I</smiles>

$16 s^{\prime}$<smiles>CCOc1cc(C#Cc2cc3ccc4ccc(-c5c(OC)cc(OC)cc5OC)nc4c3nc2-c2c(C)c(C)c(Br)c(C)c2C)c(O[14CH2])cc1I</smiles>

16

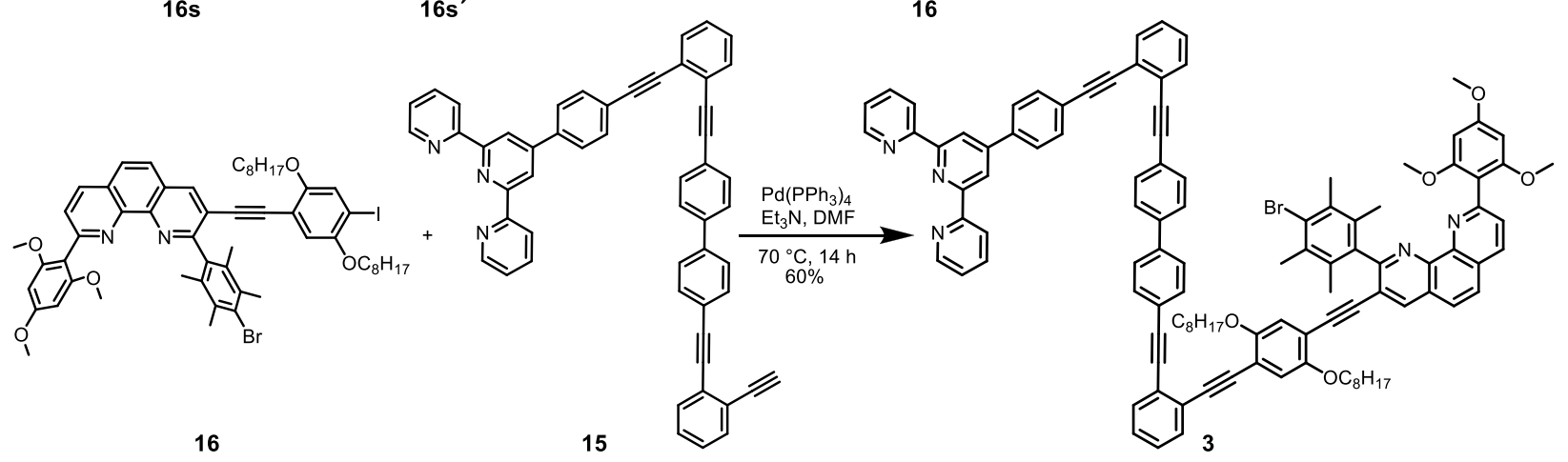

Scheme S1: Synthesis of nanoswitch 3. 

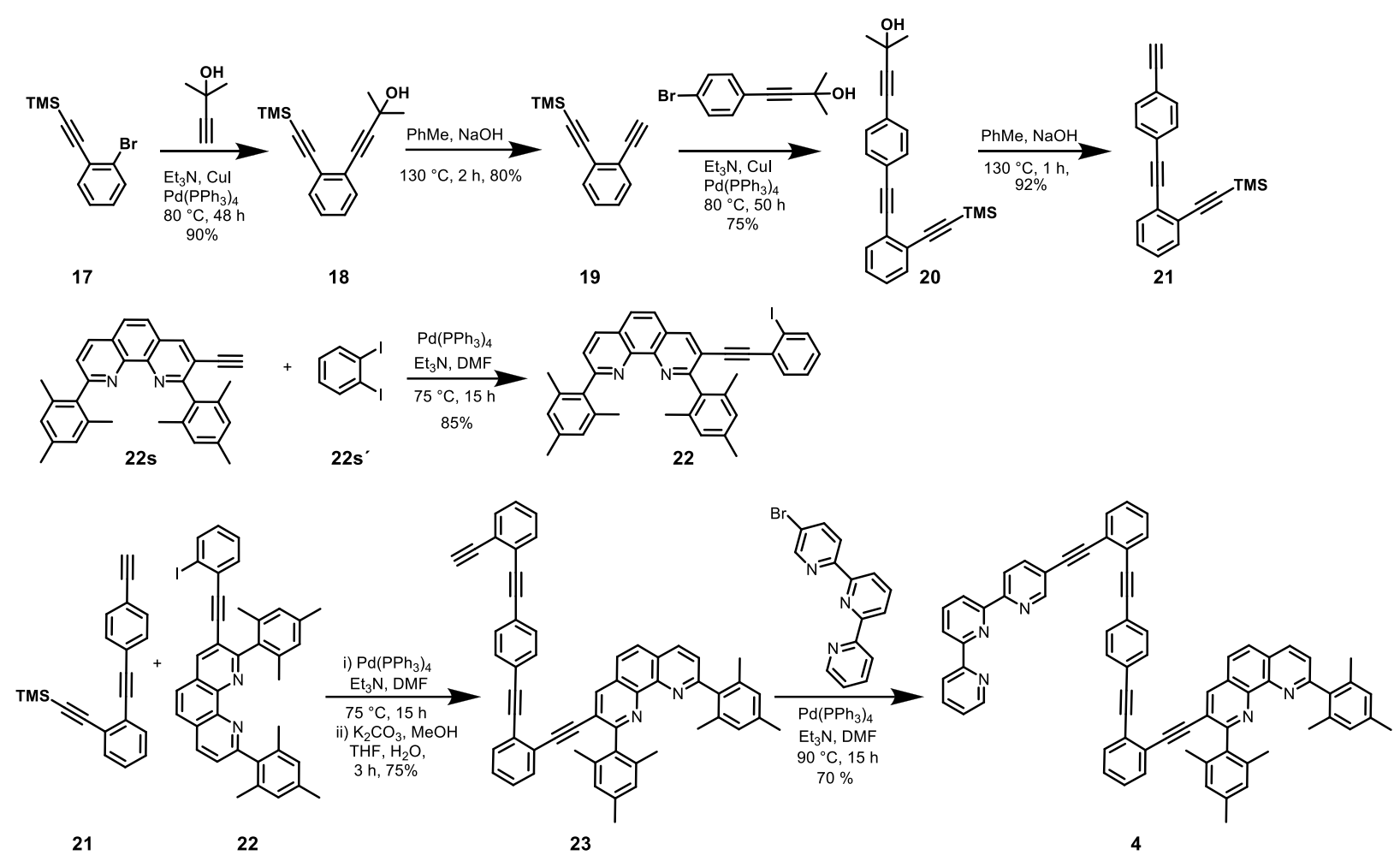

Scheme S2: Synthesis of nanoswitch 4.

\section{Synthesis of rotator 2}

Zinc(II) 5-(4-((2,6-dimethylpyridin-4-yl)ethynyl)phenyl)-10,15,20-trimesitylporphyrin (2)

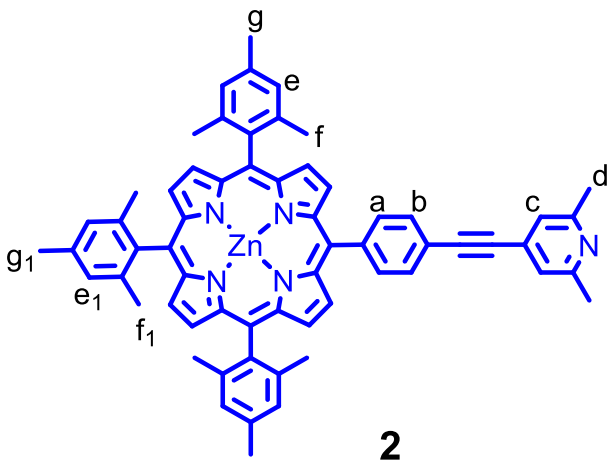

Zinc (II) 5-(4-iodophenyl)-10,15,20-trimesitylporphyrin (100 mg, $107 \mu \mathrm{mol})$ and 4-ethynyl-2,6dimethylpyridine $(70.5 \mathrm{~g}, 537 \mu \mathrm{mol})$ were dissolved in $15 \mathrm{~mL}$ of DMF and $15 \mathrm{~mL}$ of $\mathrm{Et}_{3} \mathrm{~N}$ in a sealed tube. The reaction mixture was degassed by the freeze-pump-thaw method three times. Then, $\mathrm{Pd}\left(\mathrm{PPh}_{3}\right)_{4}(24.7 \mathrm{mg}, 21.4 \mu \mathrm{mol})$ was added, a further freeze-pump-thaw cycle applied and 
the mixture allowed to stir at $75^{\circ} \mathrm{C}$ for $12 \mathrm{~h}$. All the solvents were then evaporated under vacuum. After adding dichloromethane and ice cold water, the organic part was dried over anhydrous $\mathrm{Na}_{2} \mathrm{SO}_{4}$. Firstly, the crude product was separated by column chromatography using $40 \%$ dichloromethane/hexane $\left(R_{\mathrm{f}}=0.40, \mathrm{SiO}_{2}, 40 \%\right.$ dichloromethane /hexane $)$ and secondly purified by size exclusion chromatography using SX-3 biobead to afford the pure compound 2 in $65 \%$ (65 mg, $70.0 \mu \mathrm{mol})$ yield. IR (KBr): $\tilde{v}=559,722,762,799,831,852,998,1062,1204,1335,1382$, 1438, 1478, 1523, 1551, 1600, 2211, 2732, 2855, 2917, $2948 \mathrm{~cm}^{-1}$. Mp. > $250{ }^{\circ} \mathrm{C}$. ${ }^{1} \mathbf{H}$ NMR $\left(\mathrm{CDCl}_{3}, 400 \mathrm{MHz}\right): \delta=1.85(\mathrm{~s}, 12 \mathrm{H}, \mathrm{f}-\mathrm{H}), 1.86\left(\mathrm{~s}, 6 \mathrm{H}, \mathrm{f}_{1}-\mathrm{H}\right), 2.58(\mathrm{~s}, 6 \mathrm{H}, \mathrm{d}-\mathrm{H}), 2.64(\mathrm{~s}, 9 \mathrm{H}$, $\left.\left[\mathrm{g}+\mathrm{g}_{1}\right]-\mathrm{H}\right), 7.24(\mathrm{~s}, 2 \mathrm{H}, \mathrm{c}-\mathrm{H}), 7.28\left(\mathrm{~s}, 6 \mathrm{H},\left[\mathrm{e}+\mathrm{e}_{1}\right]-\mathrm{H}\right), 7.92\left(\mathrm{~d},{ }^{3} \mathrm{~J}=8.0 \mathrm{~Hz}, 2 \mathrm{H}, \mathrm{b}-\mathrm{H}\right), 8.24\left(\mathrm{~d},{ }^{3} J=\right.$ $8.0 \mathrm{~Hz}, 2 \mathrm{H}, \mathrm{a}-\mathrm{H}), 8.72(\mathrm{~s}, 4 \mathrm{H}, \beta-\mathrm{H}), 8.78\left(\mathrm{~d},{ }^{3} \mathrm{~J}=5.6 \mathrm{~Hz}, 2 \mathrm{H}, \beta-\mathrm{H}\right), 8.86\left(\mathrm{~d},{ }^{3} J=5.6 \mathrm{~Hz}, 2 \mathrm{H}, \beta-\mathrm{H}\right)$ ppm. ${ }^{13} \mathbf{C}$ NMR (CDCl3, 100 MHz): $\delta=21.5,21.7$ (2C), 21.8, 24.3, 88.1, 93.1, 118.8, 118.9, 121.3, 122.2, 127.6 (2C), 130.0, 130.8, 131.1, 131.2, 131.7, 131.9, 134.5 (2C), 137.4 (2C), 138.9, 139.0, 139.3 (2C), 144.0, 149.5, 149.7, 149.9, 150.0, 157.9 ppm. Elemental analysis: Anal. Calcd for $\mathrm{C}_{62} \mathrm{H}_{53} \mathrm{~N}_{5} \mathrm{Zn}$ : C, 79.77; H, 5.72; N, 7.50. Found: C, 79.71; H, 5.63; N, 7.23. ESI-MS: m/z (\%) $933.0(100)[\mathrm{M}+\mathrm{H}]^{+}$.

\section{Synthesis of nanoswitch 3}

((2-Iodophenyl)ethynyl)trimethylsilane $(\mathbf{8})^{1}$

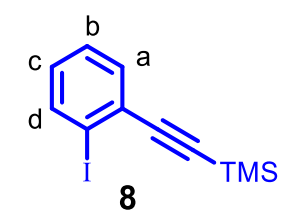

In a sealed tube, 1,2-diiodobenzene $(10.0 \mathrm{~g}, 30.3 \mathrm{mmol})$ was dissolved in $200 \mathrm{~mL}$ of Et $3 \mathrm{~N}$. The solution was deaerated for 30 min with a continuous flow of nitrogen gas. To this solution, $\mathrm{Pd}\left(\mathrm{PPh}_{3}\right)_{2} \mathrm{Cl}_{2}(213 \mathrm{mg}, 303 \mu \mathrm{mol})$ and $\mathrm{CuI}(57.7 \mathrm{mg}, 303 \mu \mathrm{mol})$ were added. Finally trimethylsilylacetylene $(6.50 \mathrm{~mL}, 45.4 \mathrm{mmol})$ was added and the mixture stirred for $3 \mathrm{~h}$ at room temperature. After evaporation of the solvent in vacuum, the product was separated $(5.00 \mathrm{~g}, 16.7$ mmol, $55 \%)$ by column chromatography on silica gel using hexane as eluent $\left(R_{\mathrm{f}}=0.40, \mathrm{SiO}_{2}, n\right.$ hexane). ${ }^{1} \mathbf{H}$ NMR (CDCl3, $\left.400 \mathrm{MHz}\right): \delta=0.28$ (s, 9H, TMS-H), 6.99 (td, ${ }^{3} J=8.0 \mathrm{~Hz},{ }^{4} \mathrm{~J}=1.6$ 
$\mathrm{Hz}, 1 \mathrm{H}, \mathrm{b}-\mathrm{H}), 7.28\left(\mathrm{td},{ }^{3} \mathrm{~J}=8.0 \mathrm{~Hz},{ }^{4} \mathrm{~J}=1.6 \mathrm{~Hz}, 1 \mathrm{H}, \mathrm{c}-\mathrm{H}\right), 7.47\left(\mathrm{dd},{ }^{3} \mathrm{~J}=8.0 \mathrm{~Hz},{ }^{4} \mathrm{~J}=1.6 \mathrm{~Hz}, 1 \mathrm{H}\right.$, $\mathrm{d}-\mathrm{H}), 7.83\left(\mathrm{dd},{ }^{3} J=8.0 \mathrm{~Hz},{ }^{4} J=1.6 \mathrm{~Hz}, 1 \mathrm{H}, \mathrm{a}-\mathrm{H}\right) \mathrm{ppm}$.

4'-(4-((2-((Trimethylsilyl)ethynyl)phenyl)ethynyl)phenyl)-2,2':6',2"-terpyridine (9)

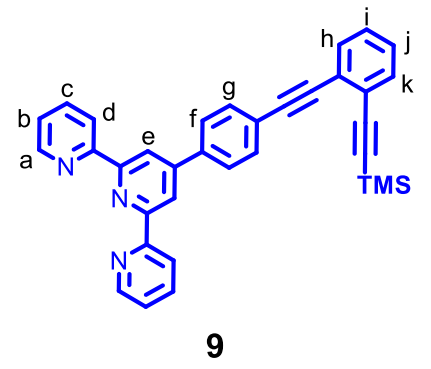

Compounds 4'-(4-ethynylphenyl)-2,2':6',2"-terpyridine (800 mg, $2.40 \mathrm{mmol})$ and 8 (1.64 g, 7.20 mmol) were placed in a sealed tube that was evacuated and filled with $\mathrm{N}_{2}$. Then freshly distilled $\mathrm{Et}_{3} \mathrm{~N}(20 \mathrm{~mL})$ and DMF $(15 \mathrm{~mL})$ were added. The solution was degassed twice by using the freezepump-thaw method. Finally, $\mathrm{Pd}\left(\mathrm{PPh}_{3}\right)_{4}(277 \mathrm{mg}, 240 \mu \mathrm{mol})$ was added to this mixture that was allowed to stir at $75{ }^{\circ} \mathrm{C}$ for $15 \mathrm{~h}$. After cooling to room temperature the solvents were removed under reduced pressure. The residue was extracted in DCM $(75 \mathrm{~mL})$ and washed with ice-cold water $(75 \mathrm{~mL})$ and brine $(30 \mathrm{~mL})$. The organic layer was dried over anhydrous $\mathrm{Na}_{2} \mathrm{SO}_{4}$ and evaporated. The column chromatographic purification of the crude product on silica gel using $40 \%$ ethyl acetate in hexane $\left(R_{\mathrm{f}}=0.30, \mathrm{SiO}_{2}, 40 \%\right.$ ethyl acetate/hexane) afforded compound 9 as a colorless solid in 58\% yield (705 mg, $1.39 \mathrm{mmol})$. IR (KBr): $\tilde{v}=621,670,758,791,843,1038$, 1093, 1208, 1248, 1386, 1411, 1442, 1467, 1514, 1566, 1584, 1603, 2158, 2214, 2852, 2920, 2954, $3057 \mathrm{~cm}^{-1}$. Mp. $>250{ }^{\circ} \mathrm{C} .{ }^{\mathbf{1}} \mathbf{H}$ NMR $\left(\mathbf{C D C l}_{3}, 400 \mathrm{MHz}\right): \delta=0.30$ (s, 9H, TMS-H), 7.27-7.34 (m, $2 \mathrm{H},[\mathrm{i}+\mathrm{j}]-\mathrm{H}), 7.37\left(\mathrm{ddd},{ }^{3} \mathrm{~J}=7.6 \mathrm{~Hz},{ }^{3} \mathrm{~J}=4.8 \mathrm{~Hz},{ }^{4} \mathrm{~J}=1.0 \mathrm{~Hz}, 2 \mathrm{H}, \mathrm{b}-\mathrm{H}\right), 7.52-7.58$ (m, 2H, [h+k]H), $7.71\left(\mathrm{~d},{ }^{3} J=8.4 \mathrm{~Hz}, 2 \mathrm{H}, \mathrm{g}-\mathrm{H}\right), 7.89\left(\mathrm{td},{ }^{3} J=7.6 \mathrm{~Hz},{ }^{4} J=1.8 \mathrm{~Hz}, 2 \mathrm{H}, \mathrm{c}-\mathrm{H}\right), 7.93\left(\mathrm{~d},{ }^{3} J=8.4\right.$ $\mathrm{Hz}, 2 \mathrm{H}, \mathrm{f}-\mathrm{H}), 8.69$ (ddd, $\left.{ }^{3} J=7.6 \mathrm{~Hz},{ }^{4} J=1.0 \mathrm{~Hz},{ }^{5} J=1.0 \mathrm{~Hz}, 2 \mathrm{H}, \mathrm{d}-\mathrm{H}\right), 8.75\left(\mathrm{ddd},{ }^{3} J=4.8 \mathrm{~Hz},{ }^{4} J\right.$ $\left.=1.8 \mathrm{~Hz},{ }^{5} \mathrm{~J}=1.0 \mathrm{~Hz}, 2 \mathrm{H}, \mathrm{a}-\mathrm{H}\right), 8.76(\mathrm{~s}, 2 \mathrm{H}, \mathrm{e}-\mathrm{H}) \mathrm{ppm} .{ }^{13} \mathbf{C} \mathbf{N M R}\left(\mathbf{C D C l}_{3}, 100 \mathrm{MHz}\right): \delta=0.07$, 89.6, 93.1, 98.8, 103.4, 118.6, 121.3, 123.9, 124.1, 125.7, 125.9, 127.2, 128.0, 128.2, 131.7, 132.2, 132.3, 136.9, 138.2, 149.1, 149.4, 156.0, 156.1 ppm. Elemental analysis: Anal. Calcd. for $\mathrm{C}_{34} \mathrm{H}_{27} \mathrm{~N}_{3} \mathrm{Si}$ : C, 80.75; H, 5.38; N, 8.31. Found: C, 81.01; H, 5.55; N, 8.10. ESI-MS: m/z (\%) 506.7 (100) $[\mathrm{M}+\mathrm{H}]^{+}$. 


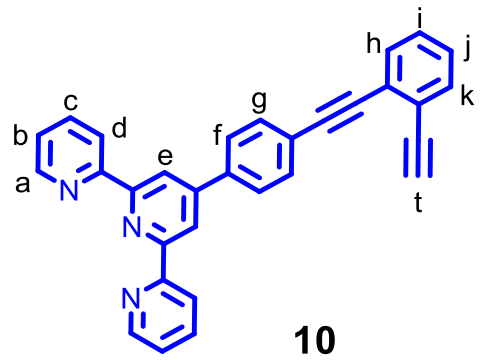

Compound 9 (600 mg, $1.19 \mathrm{mmol})$ was dissolved in $30 \mathrm{~mL}$ of THF. To this solution, $\mathrm{K}_{2} \mathrm{CO}_{3}(820$ $\mathrm{mg}, 5.93 \mathrm{mmol}$ ) dissolved in $20 \mathrm{~mL}$ of water and $30 \mathrm{~mL}$ of methanol was added. The reaction mixture was stirred at $\mathrm{rt}$ for $2 \mathrm{~h}$. The organic solvents were evaporated under vacuum. The crude was then extracted with dichloromethane. It was passed through a small pad of $\mathrm{SiO}_{2}$ using $40 \%$ ethyl acetate/hexane furnishing the colorless solid compound $\mathbf{1 0}$ in $92 \%$ yield (475 mg, 1.09 mmol). IR (KBr): $\tilde{v}=615,788,843,1038,1085,1209,1250,1385,1410,1445,1467,1515$, 1567, 1585, 1605, 2160, 2215, 2850, 2920, 2952, $3055 \mathrm{~cm}^{-1}$. Mp. > $250{ }^{\circ} \mathrm{C} .{ }^{1} \mathbf{H}$ NMR $\left(\mathbf{C D C l}_{3}\right.$, $400 \mathrm{MHz}): \delta=3.43(\mathrm{~s}, 1 \mathrm{H}, \mathrm{t}-\mathrm{H}), 7.30\left(\mathrm{td},{ }^{3} J=7.6 \mathrm{~Hz},{ }^{4} J=1.6 \mathrm{~Hz}, 1 \mathrm{H},[\mathrm{i} / \mathrm{j}]-\mathrm{H}\right), 7.32-7.37(\mathrm{~m}, 3 \mathrm{H}$, $[\mathrm{b}+\mathrm{j} / \mathrm{i}]-\mathrm{H}), 7.54-7.58(\mathrm{~m}, 2 \mathrm{H},[\mathrm{h}+\mathrm{k}]-\mathrm{H}), 7.70\left(\mathrm{~d},{ }^{3} \mathrm{~J}=8.4 \mathrm{~Hz}, 2 \mathrm{H}, \mathrm{g}-\mathrm{H}\right), 7.86\left(\mathrm{td},{ }^{3} \mathrm{~J}=8.0 \mathrm{~Hz},{ }^{4} J=\right.$ $1.8 \mathrm{~Hz}, 2 \mathrm{H}, \mathrm{c}-\mathrm{H}), 7.90\left(\mathrm{~d},{ }^{3} \mathrm{~J}=8.4 \mathrm{~Hz}, 2 \mathrm{H}, \mathrm{f}-\mathrm{H}\right), 8.66\left(\mathrm{ddd},{ }^{3} \mathrm{~J}=8.0 \mathrm{~Hz},{ }^{4} \mathrm{~J}=1.0 \mathrm{~Hz},{ }^{5} \mathrm{~J}=1.0 \mathrm{~Hz}\right.$, $2 \mathrm{H}, \mathrm{d}-\mathrm{H}), 8.73\left(\mathrm{ddd},{ }^{3} \mathrm{~J}=4.8 \mathrm{~Hz},{ }^{4} \mathrm{~J}=1.8 \mathrm{~Hz},{ }^{5} \mathrm{~J}=1.0 \mathrm{~Hz}, 2 \mathrm{H}, \mathrm{a}-\mathrm{H}\right), 8.74(\mathrm{~s}, 2 \mathrm{H}, \mathrm{e}-\mathrm{H}) \mathrm{ppm} .{ }^{13} \mathrm{C}$ NMR (CDCl3, 100 MHz): $\delta=81.3,82.1,89.3,93.2,118.6,121.3,123.8,124.6,126.1,127.2$, 128.0, 128.5, 131.8, 132.3, 132.6, 136.8, 138.3, 149.2 (2C), 149.3, 155.9, 156.0 ppm. Elemental analysis: Anal. Calcd. for $\mathrm{C}_{31} \mathrm{H}_{19} \mathrm{~N}_{3} \bullet 0.5 \mathrm{H}_{2} \mathrm{O}: \mathrm{C}, 84.14 ; \mathrm{H}, 4.56 ; \mathrm{N}, 9.49$. Found: $\mathrm{C}, 84.41 ; \mathrm{H}, 4.95$; N, 9.10. ESI-MS: $m / z(\%) 434.5(100)[\mathrm{M}+\mathrm{H}]^{+}$.

((4'-Bromo-[1,1'-biphenyl]-4-yl)ethynyl)trimethylsilane $(\mathbf{1 1})^{2}$

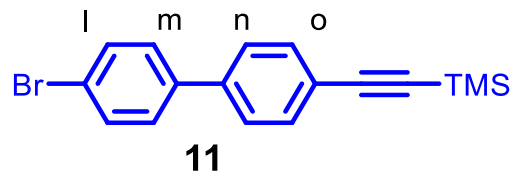

In a sealed tube, 4,4'-dibromobiphenyl (3.56 g, $11.4 \mathrm{mmol})$ was dissolved in $30 \mathrm{~mL}$ of THF and $50 \mathrm{~mL}$ of diisopropylamine. It was deaerated for 20 min using $\mathrm{N}_{2}$ gas flow. To this solution, 
$\mathrm{Pd}\left(\mathrm{Ph}_{3}\right)_{2} \mathrm{Cl}_{2}(800 \mathrm{mg}, 1.14 \mathrm{mmol})$ and $\mathrm{CuI}(217 \mathrm{mg}, 1.14 \mathrm{mmol})$ were added and finally trimethylsilylacetylene $(1.80 \mathrm{~mL}, 12.6 \mathrm{mmol})$. Then it was stirred at $60{ }^{\circ} \mathrm{C}$ for $12 \mathrm{~h}$. All the solvents were evaporated under vacuum. The crude mixture was worked up using dichloromethane and water. The organic solution was evaporated and the product was separated by column chromatography $\left(R_{\mathrm{f}}=0.60, \mathrm{SiO}_{2}\right.$, hexane $)$ using hexane as eluent with $60 \%$ yield $(2.25 \mathrm{~g}, 6.84$ mmol). ${ }^{1} \mathbf{H}$ NMR (CDCl3, 400 MHz): $\delta=0.28$ (s, 9H, TMS-H), 7.44 (d, $\left.{ }^{3} J=8.8 \mathrm{~Hz}, 2 \mathrm{H}, 1-\mathrm{H}\right)$, $7.49\left(\mathrm{~d},{ }^{3} J=8.8 \mathrm{~Hz}, 2 \mathrm{H}, \mathrm{m}-\mathrm{H}\right), 7.53\left(\mathrm{~d},{ }^{3} J=8.8 \mathrm{~Hz}, 2 \mathrm{H}, \mathrm{n}-\mathrm{H}\right), 7.56\left(\mathrm{~d},{ }^{3} J=8.8 \mathrm{~Hz}, 2 \mathrm{H}, \mathrm{o}-\mathrm{H}\right) \mathrm{ppm}$. ${ }^{13} \mathrm{C}$ NMR (CDCl3, $\left.100 \mathrm{MHz}\right): \delta=0.04,95.2,104.7,121.9,122.4,126.6,128.6,131.9,132.5$, 139.2, 139.9 ppm.

4'-(4-((2-((4'-((Trimethylsilyl)ethynyl)-[1,1'-biphenyl]-4-yl)ethynyl)phenyl)ethynyl)phenyl)2,2':6',2"-terpyridine (12)

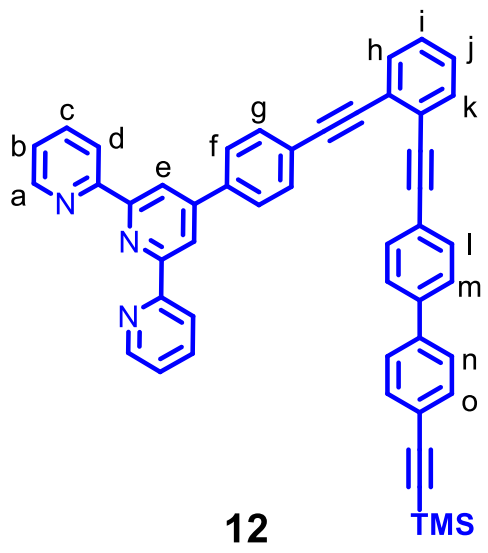

Compounds 10 (800 mg, $1.84 \mathrm{mmol})$ and 11 (3.03 g, $9.22 \mathrm{mmol})$ were dissolved in $15 \mathrm{~mL}$ of DMF and $15 \mathrm{~mL}$ of $\mathrm{Et}_{3} \mathrm{~N}$ in a sealed tube. The reaction mixture was degassed by freeze-pump-thaw method three times. Then, $\mathrm{Pd}\left(\mathrm{PPh}_{3}\right)_{4}(212 \mathrm{mg}, 184 \mathrm{nmol})$ was added. After a further freeze-pumpthaw treatment, the mixture was allowed to stir at $85^{\circ} \mathrm{C}$ for $20 \mathrm{~h}$. All the solvents were then evaporated under vacuum. The crude product was worked up with dichloromethane and ice cold water. The organic part was dried over anhydrous $\mathrm{Na}_{2} \mathrm{SO}_{4}$. Finally, the product was separated by column chromatography using $30 \%$ ethyl acetate/hexane $\left(R_{\mathrm{f}}=0.30, \mathrm{SiO}_{2}, 30 \%\right.$ ethyl acetate /hexane) furnishing the colorless solid in $65 \%(815 \mathrm{mg}, 1.20 \mathrm{mmol})$ yield. IR (KBr): $\tilde{v}=660$, $822,840,758,792,863,1035,1249,1384,1468,1493,1514,1567,1585,2027,2157,2214,2310$, 
2853, 2924, $2958 \mathrm{~cm}^{-1}$. Mp. $>250{ }^{\circ} \mathrm{C} .{ }^{1} \mathbf{H}$ NMR (CDCl, $\left.400 \mathrm{MHz}\right): \delta=0.26$ (s, 9H, TMS-H), 7.34-7.39 (m, 4H, [i+j+b]-H), $7.53\left(\mathrm{~d},{ }^{3} J=8.4 \mathrm{~Hz}, 2 \mathrm{H}, \mathrm{o}-\mathrm{H}\right), 7.55-7.62(\mathrm{~m}, 6 \mathrm{H},[\mathrm{h}+\mathrm{k}+\mathrm{n}+\mathrm{o}]-\mathrm{H})$, $7.63\left(\mathrm{~d},{ }^{3} J=8.4 \mathrm{~Hz}, 2 \mathrm{H}, \mathrm{m}-\mathrm{H}\right), 7.67\left(\mathrm{~d},{ }^{3} J=8.4 \mathrm{~Hz}, 2 \mathrm{H}, 1-\mathrm{H}\right), 7.73\left(\mathrm{~d},{ }^{3} J=8.4 \mathrm{~Hz}, 2 \mathrm{H}, \mathrm{g}-\mathrm{H}\right), 7.90$ $\left(\mathrm{td},{ }^{3} \mathrm{~J}=8.0 \mathrm{~Hz},{ }^{4} \mathrm{~J}=2.0 \mathrm{~Hz}, 2 \mathrm{H}, \mathrm{c}-\mathrm{H}\right), 7.93\left(\mathrm{~d},{ }^{3} \mathrm{~J}=8.4 \mathrm{~Hz}, 2 \mathrm{H}, \mathrm{f}-\mathrm{H}\right), 8.69$ (ddd, ${ }^{3} \mathrm{~J}=8.0 \mathrm{~Hz},{ }^{4} \mathrm{~J}=$ $\left.1.0 \mathrm{~Hz},{ }^{5} \mathrm{~J}=0.8 \mathrm{~Hz}, 2 \mathrm{H}, \mathrm{d}-\mathrm{H}\right), 8.74\left(\mathrm{ddd},{ }^{3} \mathrm{~J}=4.8 \mathrm{~Hz},{ }^{4} \mathrm{~J}=2.0 \mathrm{~Hz},{ }^{5} \mathrm{~J}=0.8 \mathrm{~Hz}, 2 \mathrm{H}, \mathrm{a}-\mathrm{H}\right), 8.76$ (s,

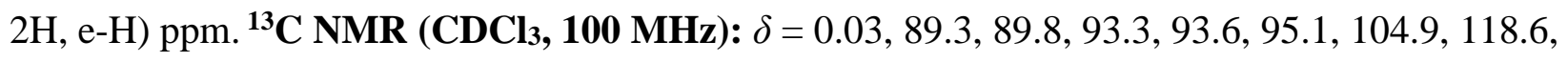
$121.4,122.4,122.6,123.9,124.1,125.7,125.9,126.8,127.0,127.3,128.1,128.2,131.8(2 \mathrm{C})$, $132.1,132.2,132.5$ (2C), 136.9, 138.3, 140.2, 149.1, 149.3, 156.0, 156.1 ppm. Elemental analysis: Anal. Calcd for $\mathrm{C}_{48} \mathrm{H}_{35} \mathrm{~N}_{3} \mathrm{Si} \bullet 0.3 \mathrm{CH}_{2} \mathrm{Cl}_{2}: \mathrm{C}, 82.01 ; \mathrm{H}, 5.07 ; \mathrm{N}, 5.94$. Found: $\mathrm{C}, 82.18 ; \mathrm{H}$, 4.70; N, 5.69. ESI-MS: $m / z(\%) 682.7(100)[\mathrm{M}+\mathrm{H}]^{+}$.

4'-(4-((2-((4'-Ethynyl-[1,1'-biphenyl]-4-yl)ethynyl)phenyl)ethynyl)phenyl)-2,2':6',2"terpyridine(13)

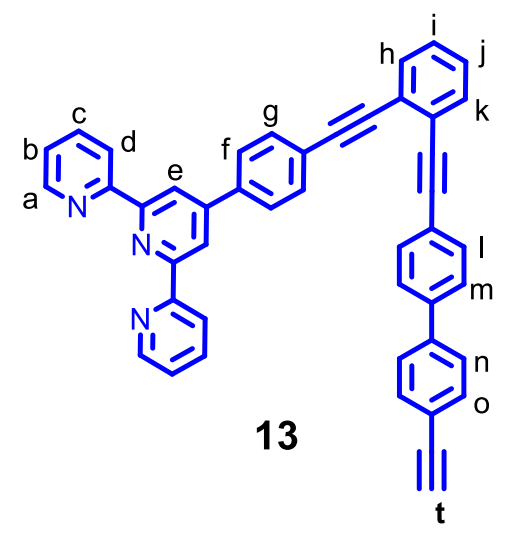

Compound 12 ( $400 \mathrm{mg}, 587 \mu \mathrm{mol}$ ) was dissolved in $30 \mathrm{~mL}$ of THF. To this solution $\mathrm{K}_{2} \mathrm{CO}_{3}$ (405 $\mathrm{mg}, 2.93 \mathrm{mmol}$ ) dissolved in $20 \mathrm{~mL}$ of water and $30 \mathrm{~mL}$ of methanol was added. The reaction mixture was stirred at $\mathrm{rt}$ for $4 \mathrm{~h}$. The organic solvents were evaporated under vacuum. The crude was then extracted with dichloromethane. It was passed through a small pad of $\mathrm{SiO}_{2}$ using $40 \%$ ethyl acetate/hexane giving a colorless solid compound $\mathbf{1 3}$ in 90\% yield (322 mg, $528 \mu \mathrm{mol}$ ). IR $(\mathrm{KBr}): \tilde{v}=543,625,758,792,823,1004,1038,1116,1164,1267,1384,1410,1567,1584,1635$, 1710, 2027, 2341, 2364, 2852, 2926, $2965 \mathrm{~cm}^{-1}$. Mp. > $250{ }^{\circ} \mathrm{C} .{ }^{1} \mathbf{H}$ NMR (CDCl, $\left.400 \mathrm{MHz}\right): \delta$ $=3.14(\mathrm{~s}, 1 \mathrm{H}, \mathrm{t}-\mathrm{H}), 7.33-7.38(\mathrm{~m}, 4 \mathrm{H},[\mathrm{i}+\mathrm{j}+\mathrm{b}]-\mathrm{H}), 7.58-7.61(\mathrm{~m}, 6 \mathrm{H},[\mathrm{h}+\mathrm{k}+\mathrm{n}+\mathrm{o}]-\mathrm{H}), 7.62\left(\mathrm{~d},{ }^{3} \mathrm{~J}=\right.$ $8.4 \mathrm{~Hz}, 2 \mathrm{H}, \mathrm{m}-\mathrm{H}), 7.67\left(\mathrm{~d},{ }^{3} \mathrm{~J}=8.4 \mathrm{~Hz}, 2 \mathrm{H}, 1-\mathrm{H}\right), 7.73\left(\mathrm{~d},{ }^{3} \mathrm{~J}=8.4 \mathrm{~Hz}, 2 \mathrm{H}, \mathrm{g}-\mathrm{H}\right), 7.87\left(\mathrm{td},{ }^{3} \mathrm{~J}=8.0\right.$ $\left.\mathrm{Hz},{ }^{4} J=2.0 \mathrm{~Hz}, 2 \mathrm{H}, \mathrm{c}-\mathrm{H}\right), 7.92\left(\mathrm{~d},{ }^{3} J=8.4 \mathrm{~Hz}, 2 \mathrm{H}, \mathrm{f}-\mathrm{H}\right), 8.68\left(\mathrm{ddd},{ }^{3} J=8.0 \mathrm{~Hz},{ }^{4} J=1.0 \mathrm{~Hz},{ }^{5} J=\right.$ 
$0.8 \mathrm{~Hz}, 2 \mathrm{H}, \mathrm{d}-\mathrm{H}), 8.73\left(\mathrm{ddd},{ }^{3} J=4.8 \mathrm{~Hz},{ }^{4} \mathrm{~J}=2.0 \mathrm{~Hz},{ }^{5} \mathrm{~J}=0.8 \mathrm{~Hz}, 2 \mathrm{H}, \mathrm{a}-\mathrm{H}\right), 8.76(\mathrm{~s}, 2 \mathrm{H}, \mathrm{e}-\mathrm{H})$ ppm. ${ }^{13} \mathbf{C}$ NMR (CDCl3, 100 MHz): $\delta=78.0,83.5,89.3,89.8,93.3,93.5,118.6,121.2,121.3$, $122.6,123.9,124.0,125.7,125.8,126.8,126.9,127.0,127.3,128.1,128.2,131.8,132.1,132.2$, 132.6, 136.9, 138.2, 140.0, 140.5, 149.1, 149.2, 156.0, 156.1 ppm. Elemental analysis: Anal. Calcd for $\mathrm{C}_{45} \mathrm{H}_{27} \mathrm{~N}_{3} \bullet 0.3 \mathrm{CH}_{2} \mathrm{Cl}_{2}$ : C, 85.66; H, 4.38; N, 6.62. Found: C, 85.99; H, 3.97; N, 6.41. ESI-MS: $m / z(\%) 610.8(100)[\mathrm{M}+\mathrm{H}]^{+}$.

4'-(4-((2-((4'-((2-((Trimethylsilyl)ethynyl)phenyl)ethynyl)-[1,1'-biphenyl]-4yl)ethynyl)phenyl)ethynyl)phenyl)-2,2':6',2"-terpyridine(14)

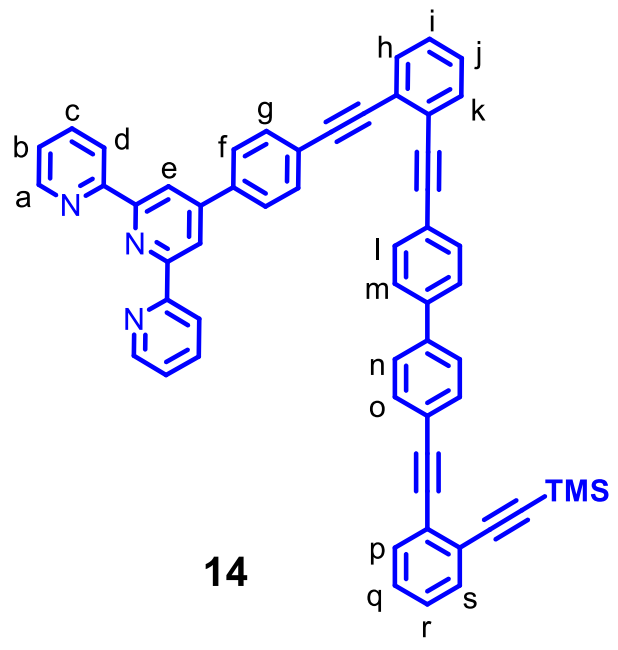

Compounds $13(250 \mathrm{mg}, 410 \mu \mathrm{mol})$ and $\mathbf{8}(246 \mathrm{mg}, 820 \mu \mathrm{mol})$ were placed in a sealed tube. It was evacuated and filled with $\mathrm{N}_{2}$, then freshly distilled $\mathrm{Et}_{3} \mathrm{~N}(20 \mathrm{~mL})$ and DMF (15 mL) were added. The solution was degassed twice by using the freeze-pump-thaw method. To this mixture $\mathrm{Pd}\left(\mathrm{PPh}_{3}\right)_{4}(47.4 \mathrm{mg}, 41.0 \mathrm{nmol})$ was added and it was allowed to stir at $75^{\circ} \mathrm{C}$ for $15 \mathrm{~h}$. The mixture was cooled to $\mathrm{rt}$ and solvents were removed under reduced pressure. The residue was extracted into DCM $(75 \mathrm{~mL})$ and washed with ice-cold water $(75 \mathrm{~mL})$ and brine $(30 \mathrm{~mL})$. The organic layer was dried over anhydrous $\mathrm{Na}_{2} \mathrm{SO}_{4}$ and evaporated. The column chromatographic purification on silica gel using $40 \%$ ethyl acetate in hexane $\left(R_{\mathrm{f}}=0.40, \mathrm{SiO}_{2}, 40 \%\right.$ ethyl acetate/hexane $)$ afforded compound 14 as colorless solid in 60\% yield (192 mg, $246 \mu \mathrm{mol})$. IR (KBr): $\tilde{v}=543,692,721$, 757, 842, 1096, 1120, 1158, 1249, 1384, 1412, 1437, 1499, 1500, 1567, 1586, 1607, 1636, 1713, 2026, 2147, 2213, 2852, 2926, $2955 \mathrm{~cm}^{-1}$. Mp. $>250{ }^{\circ} \mathrm{C} .{ }^{\mathbf{1}} \mathbf{H} \mathbf{N M R}\left(\mathbf{C D C l}_{3}, 400 \mathrm{MHz}\right): \delta=0.28$, (s, 9H, TMS-H), 7.27-7.31 (m, 2H, [q+r]-H), 7.34-7.38 (m, 4H, [i+j+b]-H), 7.50-7.54 (m, 2H, 
[s+p]-H), 7.60-7.62 (m, 2H, [h+k]-H), $7.63\left(\mathrm{~d},{ }^{3} J=8.0 \mathrm{~Hz}, 2 \mathrm{H}, \mathrm{o}-\mathrm{H}\right), 7.64\left(\mathrm{~d},{ }^{3} J=8.0 \mathrm{~Hz}, 2 \mathrm{H}, \mathrm{n}-\right.$ H), $7.69\left(\mathrm{~d},{ }^{3} J=8.0 \mathrm{~Hz}, 2 \mathrm{H}, \mathrm{m}-\mathrm{H}\right), 7.70\left(\mathrm{~d},{ }^{3} J=8.0 \mathrm{~Hz}, 2 \mathrm{H}, 1-\mathrm{H}\right), 7.74\left(\mathrm{~d},{ }^{3} J=8.4 \mathrm{~Hz}, 2 \mathrm{H}, \mathrm{g}-\mathrm{H}\right)$, $7.89\left(\mathrm{td},{ }^{3} J=8.0 \mathrm{~Hz},{ }^{3} J=1.8 \mathrm{~Hz}, 2 \mathrm{H}, \mathrm{c}-\mathrm{H}\right), 7.94\left(\mathrm{~d},{ }^{3} J=8.4 \mathrm{~Hz}, 2 \mathrm{H}, \mathrm{f}-\mathrm{H}\right), 8.68\left(\mathrm{ddd},{ }^{3} J=8.0 \mathrm{~Hz}\right.$, $\left.{ }^{4} J=1.0 \mathrm{~Hz},{ }^{5} \mathrm{~J}=0.8 \mathrm{~Hz}, 2 \mathrm{H}, \mathrm{d}-\mathrm{H}\right), 8.74\left(\mathrm{ddd},{ }^{3} J=4.8 \mathrm{~Hz},{ }^{4} J=1.8 \mathrm{~Hz},{ }^{5} \mathrm{~J}=0.8 \mathrm{~Hz}, 2 \mathrm{H}, \mathrm{a}-\mathrm{H}\right), 8.76$ (s, 2H, e-H) ppm. ${ }^{13} \mathbf{C}$ NMR (CDCl $\left.\mathbf{3}, \mathbf{1 0 0} \mathbf{M H z}\right): \delta=0.04,89.1,89.3,89.8,93.3,93.4,93.6,98.6$, 103.4, 118.6, 121.4, 122.5, 122.6, 123.9, 124.1, 125.6, 125.7, 125.9, 126.0, 126.9, 127.0, 127.3, 127.9, 128.1, 128.2 (2C), 131.7, 131.8, 131.9, 132.1, 132.2 (2C), 132.3, 136.9, 138.3, 140.1, 140.2, 149.1, 149.3, 156.0, 156.1 ppm. Elemental analysis: Anal. Calcd for $\mathrm{C}_{56} \mathrm{H}_{39} \mathrm{~N}_{3} \mathrm{Si}$ : C, 86.01; H, 5.03; N, 5.37. Found: C, 85.99; H, 5.37; N, 5.50. ESI-MS: $m / z(\%) 783.0(100)[\mathrm{M}+\mathrm{H}]^{+}$.

4'-(4-((2-((4'-((2-Ethynylphenyl)ethynyl)-[1,1'-biphenyl]-4-yl)ethynyl)phenyl)ethynyl)phenyl)$2,2^{\prime}: 6^{\prime}, 2^{\prime \prime}$-terpyridine $(\mathbf{1 5})$

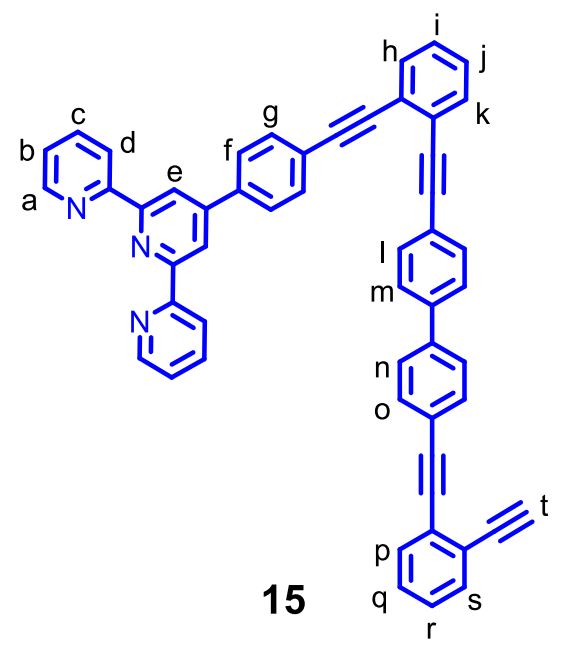

Compound 14 (170 mg, $217 \mu \mathrm{mol})$ was dissolved in $30 \mathrm{~mL}$ of THF. To this solution $\mathrm{K}_{2} \mathrm{CO}_{3}$ (405 $\mathrm{mg}, 2.93 \mathrm{mmol}$ ) dissolved in $20 \mathrm{~mL}$ of water and $30 \mathrm{~mL}$ of methanol was added. The reaction mixture was stirred at $\mathrm{rt}$ for $2 \mathrm{~h}$. The organic solvents were evaporated under vacuum. The crude product was then extracted with dichloromethane. The solution was passed through a small pad of $\mathrm{SiO}_{2}$ using $40 \%$ ethyl acetate/hexane $\left(R_{\mathrm{f}}=0.40, \mathrm{SiO}_{2}, 40 \%\right.$ ethyl acetate/hexane $)$ giving the colorless solid compound $\mathbf{1 5}$ in 90\% yield (139 mg, $195 \mu \mathrm{mol})$. IR $(\mathbf{K B r}): \tilde{v}=519,620,660$, 718, 757, 821, 868, 998, 1039, 1158, 1248, 1309, 1386, 1411, 1435, 1467, 1481, 1514, 1556, 1584, 1603, 1638, 2152, 2216, 2350, 2956, $3052 \mathrm{~cm}^{-1}$. Mp. > $250{ }^{\circ} \mathrm{C} .{ }^{1} \mathbf{H}$ NMR (CDCl, $\left.400 \mathrm{MHz}\right): \delta$ $=3.38(\mathrm{~s}, 1 \mathrm{H}, \mathrm{t}-\mathrm{H}), 7.32-7.39(\mathrm{~m}, 6 \mathrm{H},[\mathrm{q}+\mathrm{i}+\mathrm{r}+\mathrm{j}+\mathrm{b}]-\mathrm{H}), 7.53-7.57(\mathrm{~m}, 2 \mathrm{H},[\mathrm{p}+\mathrm{s}]-\mathrm{H}), 7.60-7.63(\mathrm{~m}$, 
$2 \mathrm{H},[\mathrm{h}+\mathrm{k}]-\mathrm{H}), 7.64-7.70(\mathrm{~m}, 8 \mathrm{H},[\mathrm{l}+\mathrm{m}+\mathrm{n}+\mathrm{o}]-\mathrm{H}), 7.74\left(\mathrm{~d},{ }^{3} J=8.0 \mathrm{~Hz}, 2 \mathrm{H}, \mathrm{g}-\mathrm{H}\right), 7.89\left(\mathrm{td},{ }^{3} J=8.0\right.$ $\left.\mathrm{Hz},{ }^{4} J=1.8 \mathrm{~Hz}, 2 \mathrm{H}, \mathrm{c}-\mathrm{H}\right), 7.94\left(\mathrm{~d},{ }^{3} J=8.0 \mathrm{~Hz}, 2 \mathrm{H}, \mathrm{f}-\mathrm{H}\right), 8.69\left(\mathrm{ddd},{ }^{3} J=8.0 \mathrm{~Hz},{ }^{4} J=1.2 \mathrm{~Hz},{ }^{5} \mathrm{~J}=\right.$ $1.2 \mathrm{~Hz}, 2 \mathrm{H}, \mathrm{d}-\mathrm{H}$ ), 8.74 (ddd, ${ }^{3} J=4.8 \mathrm{~Hz},{ }^{4} J=1.8 \mathrm{~Hz},{ }^{5} J=1.2 \mathrm{~Hz}, 2 \mathrm{H}, \mathrm{a}-\mathrm{H}$ ), 8.76 (s, $2 \mathrm{H}, \mathrm{e}-\mathrm{H}$ ) ppm. ${ }^{13} \mathbf{C}$ NMR (CDCl3, 100 MHz): $\delta=81.2,82.2,88.8,89.3,89.8,93.3,93.4,93.6,118.6,121.3$, 122.3, 122.5, 123.9, 124.6, 125.6, 125.9, 126.2, 126.9, 127.0, 127.3, 127.9, 128.1, 128.2, 128.4, $128.5,131.7,131.8,131.9,132.0,132.1$ (2C), 132.2, 132.6, 136.9, 138.2, 140.2, 149.1, 149.2, 156.0, $156.1 \mathrm{ppm}$. Elemental analysis: Anal. Calcd for $\mathrm{C}_{53} \mathrm{H}_{31} \mathrm{~N}_{3} \bullet 0.9 \mathrm{CH}_{2} \mathrm{Cl}_{2}: \mathrm{C}, 82.34 ; \mathrm{H}, 4.20$; N, 5.34. Found: C, 82.18; H, 4.40; N, 5.39. ESI-MS: $m / z(\%) 710.9(100)[\mathrm{M}+\mathrm{H}]^{+}$.

2-(4-Bromo-2,3,5,6-tetramethylphenyl)-3-((4-ethynyl-2,5-bis(octyloxy)phenyl)ethynyl)-9-(2,4,6trimethoxyphenyl)-1,10-phenanthroline(16)

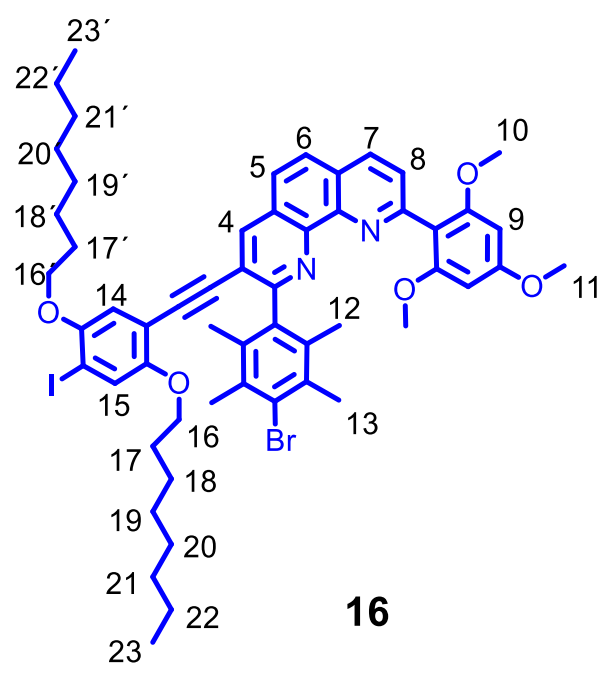

Compounds 2-(4-bromo-2,3,5,6-tetramethylphenyl)-3-ethynyl-9-(2,4,6-trimethoxyphenyl)-1,10phenanthroline ( $250 \mathrm{mg}, 430 \mu \mathrm{mol}$ ) and 1,4-diiodo-2,5-bis(octyloxy)benzene (1.26 g, $2.15 \mathrm{mmol})$ were placed in a sealed tube. It was evacuated and filled with $\mathrm{N}_{2}$, then freshly distilled $\mathrm{Et}_{3} \mathrm{~N}$ (20 $\mathrm{mL})$ and DMF $(25 \mathrm{~mL})$ were added. The solution was degassed twice by using the freeze-pumpthaw method. To this mixture, $\mathrm{Pd}\left(\mathrm{PPh}_{3}\right)_{4}(49.6 \mathrm{mg}, 43.0 \mu \mathrm{mol})$ was added and it was allowed to stir at $70{ }^{\circ} \mathrm{C}$ for $15 \mathrm{~h}$. The mixture was cooled to room temperature and solvents were removed under reduced pressure. The residue was extracted in DCM $(75 \mathrm{~mL})$ and washed with ice-cold water $(75 \mathrm{~mL})$ and brine $(30 \mathrm{~mL})$. The organic layer was dried over anhydrous $\mathrm{Na}_{2} \mathrm{SO}_{4}$ and evaporated. The column chromatographic purification of the crude product on silica gel using $25 \%$ ethyl acetate in hexane $\left(R_{\mathrm{f}}=0.30, \mathrm{SiO}_{2}, 25 \%\right.$ ethyl acetate/hexane) afforded compound $\mathbf{1 6}$ as 
colorless solid in 70\% yield (313 mg, $300 \mu \mathrm{mol})$. IR (KBr): $\tilde{v}=540,563,809,847,990,1032$, $1129,1156,1205,1222,1335,1384,1413,1457,1500,1587,1609,2023,2208,2368,2860,2828$, $2951 \mathrm{~cm}^{-1}$. Mp. $>250{ }^{\circ} \mathrm{C} .{ }^{1} \mathbf{H}$ NMR $\left(\mathbf{C D C l}_{3}, \mathbf{4 0 0} \mathbf{M H z}\right): \delta=0.87\left(\mathrm{t},{ }^{3} \mathrm{~J}=7.2 \mathrm{~Hz}, 3 \mathrm{H},\left[23 / 23^{\prime}\right]-\right.$ $\mathrm{H}), 0.90,\left(\mathrm{t},{ }^{3} J=7.2 \mathrm{~Hz}, 3 \mathrm{H},\left[23^{\prime} / 23\right]-\mathrm{H}\right), 1.27-1.34\left(\mathrm{~m}, 8 \mathrm{H},\left[21+22+21^{\prime}+22^{\prime}\right]-\mathrm{H}\right), 1.35-1.42(\mathrm{~m}$, $\left.8 \mathrm{H},\left[19+20+19^{\prime}+20^{\prime}\right]-\mathrm{H}\right), 1.48-1.58\left(\mathrm{~m}, 4 \mathrm{H},\left[18+18^{\prime}\right]-\mathrm{H}\right), 1.76-1.86\left(\mathrm{~m}, 4 \mathrm{H},\left[17+17^{\prime}\right]-\mathrm{H}\right), 2.01$ (s, 6H, 13-H), 2.43 (s, 6H, 12-H), 3.73 (s, 6H, 10-H), 3.80 (t, $\left.{ }^{3} J=6.4 \mathrm{~Hz}, 2 \mathrm{H}, 16^{\prime}-\mathrm{H}\right), 3.84$ (s, 3H, $11-\mathrm{H}), 3.93\left(\mathrm{t},{ }^{3} \mathrm{~J}=6.4 \mathrm{~Hz}, 2 \mathrm{H}, 16-\mathrm{H}\right), 6.08(\mathrm{~s}, 1 \mathrm{H}, 15-\mathrm{H}), 6.23(\mathrm{~s}, 2 \mathrm{H}, 9-\mathrm{H}), 7.25(\mathrm{~s}, 1 \mathrm{H}, 14-\mathrm{H})$, $7.66\left(\mathrm{~d},{ }^{3} J=8.4 \mathrm{~Hz}, 1 \mathrm{H}, 8-\mathrm{H}\right), 7.78\left(\mathrm{~d},{ }^{3} J=8.4 \mathrm{~Hz}, 1 \mathrm{H},[5 / 6]-\mathrm{H}\right), 7.85\left(\mathrm{~d},{ }^{3} J=8.4 \mathrm{~Hz}, 1 \mathrm{H},[6 / 5]-\right.$ H), $8.23\left(\mathrm{~d},{ }^{3} \mathrm{~J}=8.4 \mathrm{~Hz}, 1 \mathrm{H}, 7-\mathrm{H}\right), 8.41(\mathrm{~s}, 1 \mathrm{H}, 4-\mathrm{H}) \mathrm{ppm} .{ }^{13} \mathbf{C} \mathbf{~ N M R}\left(\mathbf{C D C l}_{3}, \mathbf{1 0 0} \mathbf{~ M H z}\right): \delta=14.1$ (2C), 18.6 (2C), 21.0 (2C), 22.7, 26.0, 26.1, 29.2, 29.3, 29.3, 29.4, 29.5, 31.8, 31.9, 55.4, 56.4, 69.1, 69.6, 90.1, 91.3, 91.4, 91.6, 113.6, 114.0, 116.5, 117.3, 119.9, 125.3, 126.8, 127.0, 127.3, 127.9, 128.8, 133.3, 133.8, 135.2, 138.1, 139.4, 144.9, 145.9, 152.8, 154.2, 155.4, 159.3, 161.5, 162.1 ppm. Elemental analysis: Anal. Calcd for $\mathrm{C}_{55} \mathrm{H}_{64} \mathrm{BrIN}_{2} \mathrm{O}_{5}: \mathrm{C}, 63.52 ; \mathrm{H}, 6.20 ; \mathrm{N}, 2.69$. Found: C, 63.46; H, 6.09; N, 2.36. ESI-MS: $m / z$ (\%) $1040.9(100)[\mathrm{M}+\mathrm{H}]^{+}$.

Synthesis of switch $\mathbf{3}$

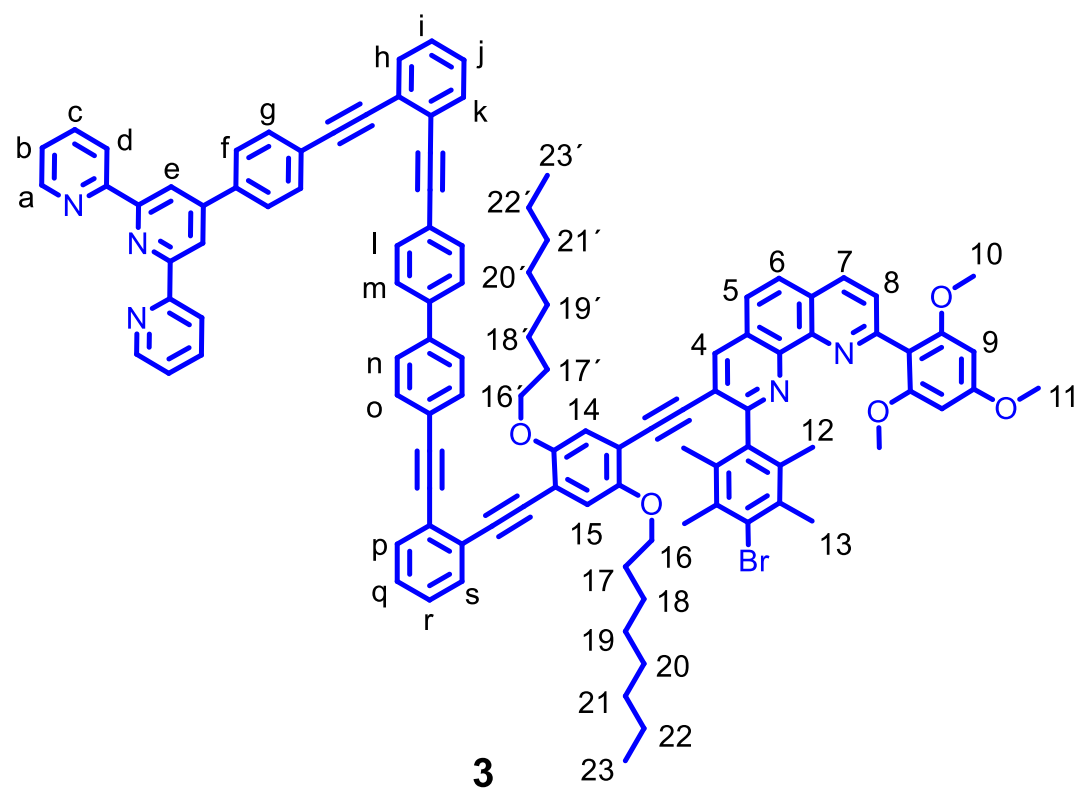

In a dry sealed tube compounds $\mathbf{1 5}(75.0 \mathrm{mg}, 106 \mu \mathrm{mol})$ and $\mathbf{1 6}(121 \mathrm{mg}, 116 \mu \mathrm{mol})$ were taken, dissolved in $15 \mathrm{~mL}$ DMF and $10 \mathrm{~mL}$ of $\mathrm{Et}_{3} \mathrm{~N}$. The solution was degassed twice by the freezepump-thaw method. To this mixture, $\mathrm{Pd}\left(\mathrm{PPh}_{3}\right)_{4}(12.3 \mathrm{mg}, 10.6 \mu \mathrm{mol})$ was added and again the 
freeze-pump-thaw method was followed once. Then the solution was allowed to stir for $14 \mathrm{~h}$ at 70 ${ }^{\circ} \mathrm{C}$. All the solvents were evaporated under vacuum. The residue was dissolved in $\mathrm{CH}_{2} \mathrm{Cl}_{2}$ and worked up with ice cold water to remove DMF. The organic part was dried over anhydrous $\mathrm{Na}_{2} \mathrm{SO}_{4}$. Purification was performed by column chromatography on $\mathrm{SiO}_{2}\left(R_{\mathrm{f}}=0.3, \mathrm{SiO}_{2}, 40 \%\right.$ ethyl acetate in hexane) using $\mathrm{CH}_{2} \mathrm{Cl}_{2}$ as eluent. It was eventually purified by size exclusion chromatography with SX-3 biobead using distilled THF as the eluent to furnishing a yellow solid in 60\% yield $(103 \mathrm{mg}, 63.6 \mu \mathrm{mol})$. IR $(\mathbf{K B r}) \tilde{v}=533,622,660,757,792,822,830,898,919$, 946, 994, 1004, 1035, 1127, 1156, 1205, 1225, 1273, 1384, 1334, 1410, 1466, 1500, 1566, 1573, 1585, 1605, 1635, 2026, 2204, 2250, 2334, 2854, 2926, $2954 \mathrm{~cm}^{-1}$. Mp. > $250{ }^{\circ} \mathrm{C}$. ${ }^{1} \mathbf{H}$ NMR $\left(\mathrm{CD}_{2} \mathbf{C l}_{2}, 400 \mathrm{MHz}\right): \delta=0.80\left(\mathrm{t},{ }^{3} \mathrm{~J}=6.8 \mathrm{~Hz}, 3 \mathrm{H},\left[23 / 23^{\prime}\right]-\mathrm{H}\right), 0.85\left(\mathrm{t},{ }^{3} \mathrm{~J}=6.8 \mathrm{~Hz}, 3 \mathrm{H},\left[23^{\prime} / 23\right]-\right.$ $\mathrm{H}), 1.18-1.34\left(\mathrm{~m}, 16 \mathrm{H},\left[22+22^{\prime}\right]-\mathrm{H}+\left[21+21^{\prime}\right]-\mathrm{H}+\left[20+20^{\prime}\right]-\mathrm{H}+\left[19+19^{\prime}\right]-\mathrm{H}\right), 1.41\left(\mathrm{t},{ }^{3} \mathrm{~J}=6.8\right.$ $\left.\mathrm{Hz}, 2 \mathrm{H}, 18^{\prime}-\mathrm{H}\right), 1.49\left(\mathrm{t},{ }^{3} \mathrm{~J}=6.8 \mathrm{~Hz}, 2 \mathrm{H}, 18-\mathrm{H}\right), 1.70\left(\mathrm{t},{ }^{3} \mathrm{~J}=6.8 \mathrm{~Hz}, 2 \mathrm{H}, 17^{\prime}-\mathrm{H}\right), 1.78\left(\mathrm{t},{ }^{3} J=6.8\right.$ $\mathrm{Hz}, 2 \mathrm{H}, 17-\mathrm{H}), 1.98(\mathrm{~s}, 6 \mathrm{H}, 13-\mathrm{H}), 2.46(\mathrm{~s}, 6 \mathrm{H}, 12-\mathrm{H}), 3.67$ (s, 6H, 10-H), $3.80\left(\mathrm{t},{ }^{3} \mathrm{~J}=6.8 \mathrm{~Hz}, 2 \mathrm{H}\right.$, $\left.16^{\prime}-\mathrm{H}\right), 3.85\left(\mathrm{t},{ }^{3} \mathrm{~J}=6.8 \mathrm{~Hz}, 2 \mathrm{H}, 16-\mathrm{H}\right), 3.86(\mathrm{~s}, 3 \mathrm{H}, 11-\mathrm{H}), 6.24$ (s, 2H, 9-H), 6.25 (s, 1H, 14-H), $6.98(\mathrm{~s}, 1 \mathrm{H}, 15-\mathrm{H}), 7.32-7.36(\mathrm{~m}, 4 \mathrm{H},[\mathrm{i}+\mathrm{j}+\mathrm{b}]-\mathrm{H}), 7.36-7.41(\mathrm{~m}, 3 \mathrm{H},[8+\mathrm{q}+\mathrm{r}]-\mathrm{H}), 7.55-7.60(\mathrm{~m}$, $2 \mathrm{H},[\mathrm{h}+\mathrm{k}]-\mathrm{H}), 7.62-7.65(\mathrm{~m}, 2 \mathrm{H},[\mathrm{s}+\mathrm{p}]-\mathrm{H}), 7.66\left(\mathrm{~d},{ }^{3} \mathrm{~J}=8.0 \mathrm{~Hz}, 2 \mathrm{H},[\mathrm{m} / \mathrm{n}]-\mathrm{H}\right), 7.67\left(\mathrm{~d},{ }^{3} J=8.0\right.$ $\mathrm{Hz}, 2 \mathrm{H},[\mathrm{n} / \mathrm{m}]-\mathrm{H}), 7.70\left(\mathrm{~d},{ }^{3} J=8.0 \mathrm{~Hz}, 1 \mathrm{H},[5 / 6]-\mathrm{H}\right), 7.71\left(\mathrm{~d},{ }^{3} \mathrm{~J}=8.0 \mathrm{~Hz}, 1 \mathrm{H},[6 / 5]-\mathrm{H}\right), 7.76-7.79$ (m, 4H, [l+o]-H), $7.84\left(\mathrm{~d},{ }^{3} J=8.0 \mathrm{~Hz}, 2 \mathrm{H}, \mathrm{g}-\mathrm{H}\right), 7.85\left(\mathrm{td},{ }^{3} J=8.0 \mathrm{~Hz},{ }^{4} J=1.8 \mathrm{~Hz}, 2 \mathrm{H}, \mathrm{c}-\mathrm{H}\right), 7.96$ $\left(\mathrm{d},{ }^{3} J=8.0 \mathrm{~Hz}, 2 \mathrm{H}, \mathrm{f}-\mathrm{H}\right), 8.25\left(\mathrm{~d},{ }^{3} J=8.0 \mathrm{~Hz}, 1 \mathrm{H}, 7-\mathrm{H}\right), 8.39$ (s, $\left.1 \mathrm{H}, 4-\mathrm{H}\right), 8.66$ (ddd, ${ }^{3} J=8.0 \mathrm{~Hz}$, $\left.{ }^{4} J=1.0 \mathrm{~Hz},{ }^{5} \mathrm{~J}=0.8 \mathrm{~Hz}, 2 \mathrm{H}, \mathrm{d}-\mathrm{H}\right), 8.70\left(\mathrm{ddd},{ }^{3} \mathrm{~J}=4.8 \mathrm{~Hz},{ }^{4} \mathrm{~J}=1.8 \mathrm{~Hz},{ }^{5} \mathrm{~J}=0.8 \mathrm{~Hz}, 2 \mathrm{H}, \mathrm{a}-\mathrm{H}\right.$ ), 8.80 (s, 2H, e-H) ppm. ${ }^{13} \mathbf{C}$ NMR ( $\left.\mathbf{C D C l}_{3}, \mathbf{1 0 0} \mathbf{~ M H z}\right): \delta=14.1$ (2C), 18.6 (2C), 21.0 (2C), 22.7, 26.0, 26.1, 29.2, 29.3, 29.3, 29.4, 29.5, 31.8, 31.8, 55.4, 56.3, 69.4, 69.4, 89.3, 89.4, 89.8, 90.3, 91.7, 92.0, 92.2, 93.3, 93.4, 93.6, 93.9, 113.6, 114.4, 116.9, 117.0, 118.6, 119.9, 121.3, 122.7, 123.9, $124.1,125.4,125.7,125.9,126.1,126.7,126.8,126.8,126.9,126.9,127.0,127.1,127.2,127.3$, $127.3,127.9,128.0,128.1,128.2,128.8,131.6,131.8,131.9,132.1,132.2,132.2,132.2,133.3$, 133.8, 135.3, 136.9, 138.2, 138.3, 139.4, 140.1, 144.8, 145.9, 149.1, 149.1, 149.3, 153.0, 153.6, 155.4, 156.0, 156.1, 159.2, 161.6, 162.1 ppm. Elemental analysis: Anal. Calcd. for $\mathrm{C}_{108} \mathrm{H}_{94} \mathrm{BrN}_{5} \mathrm{O}_{5} \bullet \mathrm{CH}_{2} \mathrm{Cl}_{2}: \mathrm{C}, 76.70 ; \mathrm{H}, 5.67 ; \mathrm{N}, 4.10$. Found: C, 76.50; H, 5.48; N, 4.11. ESI-MS: $\mathrm{m} / \mathrm{z}(\%) 1622.9(100)[\mathrm{M}+\mathrm{H}]^{+}$. 


\section{Synthesis of nanoswitch 4}

((2-Bromophenyl)ethynyl)trimethylsilane $(\mathbf{1 7})^{3}$

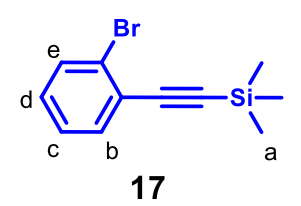

A mixture of 1-bromo-2-iodobenzene (5.00 g, $17.7 \mathrm{mmol}), \mathrm{PdCl}_{2}\left(\mathrm{PPh}_{3}\right)_{2}(124 \mathrm{mg}, 180 \mu \mathrm{mol})$ and $\mathrm{CuI}(67.0 \mathrm{mg}, 350 \mu \mathrm{mol})$ were placed in a sealed tube. The tube was evacuated and filled with $\mathrm{N}_{2}$ (3×). Pre-degassed anhydrous $\mathrm{Et}_{3} \mathrm{~N}(55 \mathrm{~mL})$ and trimethylsilylacetylene (TMSA, $1.93 \mathrm{~g}, 2.80 \mathrm{~mL}$, $19.5 \mathrm{mmol}$ ) were added under $\mathrm{N}_{2}$ atmosphere, and the reaction mixture was stirred at room temperature for $3 \mathrm{~h}$ (TLC). The mixture was filtered, washed with $n$-hexane $(30 \mathrm{~mL} \times 2)$ and the solvent was evaporated. The residue was purified by column chromatography on silica gel using $n$-hexane $\left(R_{\mathrm{f}}=0.65, \mathrm{SiO}_{2}, n\right.$-hexane) to furnish 17 as a tan oil in $90 \%$ yield $(4.03 \mathrm{~g}, 15.9 \mathrm{mmol})$. IR (neat): $\tilde{v}=3064,2960,2900,2163,1951,1584,1465,1427,1251,1119,1028,863,756,704$, 671, $549 \mathrm{~cm}^{-1} .{ }^{1} \mathbf{H}$ NMR (CDCl, $\left.400 \mathrm{MHz}\right): \delta=0.28(\mathrm{~s}, 9 \mathrm{H}, \mathrm{a}-\mathrm{H}), 7.15\left(\mathrm{td},{ }^{3} \mathrm{~J}=7.6 \mathrm{~Hz},{ }^{4} \mathrm{~J}=1.2\right.$ $\mathrm{Hz}, 1 \mathrm{H}, \mathrm{c}-\mathrm{H}), 7.24\left(\mathrm{ddd},{ }^{3} J=8.0 \mathrm{~Hz},{ }^{3} J=7.6 \mathrm{~Hz},{ }^{4} J=1.6 \mathrm{~Hz}, 1 \mathrm{H}, \mathrm{d}-\mathrm{H}\right), 7.49\left(\mathrm{dd},{ }^{3} J=7.6 \mathrm{~Hz},{ }^{4} J\right.$ $=1.6 \mathrm{~Hz}, 1 \mathrm{H}, \mathrm{b}-\mathrm{H}), 7.57\left(\mathrm{dd},{ }^{3} J=8.0 \mathrm{~Hz},{ }^{4} J=1.2 \mathrm{~Hz}, 1 \mathrm{H}, \mathrm{e}-\mathrm{H}\right) \mathrm{ppm} .{ }^{13} \mathbf{C} \mathbf{N M R}(\mathbf{C D C l}, 100$ MHz): $\delta=-0.2,99.6,103.0,125.2,125.7,126.9,129.5,132.3,133.6$ ppm.

2-Methyl-4-(2-((trimethylsilyl)ethynyl)phenyl)but-3-yn-2-ol (18 $)^{4}$

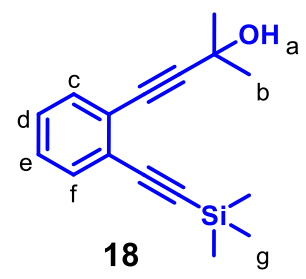

2-Methylbut-3-yn-2-ol (16.7 g, $19.2 \mathrm{~mL}, 19.8 \mathrm{mmol})$ was added to a mixture of 17 (5.00 g, 19.8 $\mathrm{mmol})$ and $\mathrm{CuI}(188 \mathrm{mg}, 987 \mu \mathrm{mol})$ in anhydrous $\mathrm{Et}_{3} \mathrm{~N}(150 \mathrm{~mL})$. The resultant mixture was purged with $\mathrm{N}_{2}$ for $45 \mathrm{~min} . \mathrm{Pd}\left(\mathrm{PPh}_{3}\right)_{4}(1.14 \mathrm{~g}, 987 \mu \mathrm{mol})$ was added and the reaction mixture was heated to $80^{\circ} \mathrm{C}$ for $48 \mathrm{~h}$ (TLC). The solvent was removed by rotary evaporation. The residue was dissolved in DCM $(150 \mathrm{~mL})$, successively washed with deionized water $(150 \mathrm{~mL} \times 3)$ and saturated brine solution $(150 \mathrm{~mL})$. The organic layer was removed, dried over anhydrous $\mathrm{Na}_{2} \mathrm{SO}_{4}$ 
and evaporated in vacuo. The dark brown residue was purified by column chromatography on silica gel using $10 \%$ EtOAc in $n$-hexane $\left(R_{\mathrm{f}}=0.44, \mathrm{SiO}_{2}, 30 \%\right.$ EtOAc in $n$-hexane) to furnish 18 as a viscous brown oil in 90\% yield (4.57 g, $17.8 \mathrm{mmol}$ ). IR (neat): $\tilde{v}=502,561,642,697,757$, 869, 958, 1032, 1152, 1248, 1368, 1505, 1597, 1644, 1682, 1835, 1935, 2149, 2220, 2977, 3056, 3446, $3579 \mathrm{~cm}^{-1} .{ }^{1} \mathbf{H}$ NMR (CDCl3, $\left.400 \mathrm{MHz}\right): \delta=0.27$ (s, 9H, g-H), 1.64 (s, 6H, b-H), 2.16 (bs, $1 \mathrm{H}, \mathrm{a}-\mathrm{H}), 7.22-7.24(\mathrm{~m}, 2 \mathrm{H},[\mathrm{d}+\mathrm{e}]-\mathrm{H}), 7.40\left(\mathrm{ddd},{ }^{3} J=7.2 \mathrm{~Hz},{ }^{4} J=2.0 \mathrm{~Hz},{ }^{5} J=0.4 \mathrm{~Hz}, 1 \mathrm{H}, \mathrm{f}-\mathrm{H}\right)$, $7.46\left(\mathrm{ddd},{ }^{3} J=7.2 \mathrm{~Hz},{ }^{4} J=2.0 \mathrm{~Hz},{ }^{5} J=0.4 \mathrm{~Hz}, 1 \mathrm{H}, \mathrm{c}-\mathrm{H}\right) \mathrm{ppm} .{ }^{13} \mathbf{C} \mathbf{~ N M R}\left(\mathbf{C D C l}_{3}, \mathbf{1 0 0} \mathbf{M H z}\right): \delta$ $=0.01,31.5,65.7,80.8,97.7,98.1,103.3,125.3,125.6,127.9,128.1,131.8,132.3 \mathrm{ppm}$.

((2-Ethynylphenyl)ethynyl)trimethylsilane (19) ${ }^{4}$

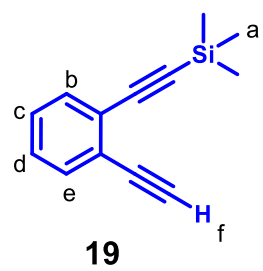

A solution of $18(1.82 \mathrm{~g}, 7.10 \mathrm{mmol})$ in anhydrous PhMe $(125 \mathrm{~mL})$ was heated in an oil bath (130 ${ }^{\circ} \mathrm{C}$ ). The reaction mixture was allowed to reflux for $\sim 10 \mathrm{~min}$, then granular $\mathrm{NaOH}(315 \mathrm{mg}, 7.81$ mmol) was added in one portion under inert atmosphere. The resulting mixture was further heated to reflux for $2 \mathrm{~h}$ (TLC). The reaction mixture was allowed to cool to room temperature, diluted with deionized water and extracted with PhMe $(50 \mathrm{~mL})$. The organic layer was washed with brine $(50 \mathrm{~mL})$ and dried over anhydrous $\mathrm{Na}_{2} \mathrm{SO}_{4}$. The solvent was removed under reduced pressure and the residue was purified by flash column chromatography $(\phi=3.5 \mathrm{~cm}, l=45 \mathrm{~cm})$ on silica gel using $n$-hexane ( $R_{\mathrm{f}}=0.60, \mathrm{SiO}_{2}, n$-hexane) to afford compound $\mathbf{1 9}$ as viscous yellow oil with $80 \%$ yield (1.13 g, $5.68 \mathrm{mmol})$. IR (neat): $\tilde{v}=509,642,759,863,1036,1096,1474,1591,1713,1957$, 2160, 2484, 2899, 2960, 3063, $3296 \mathrm{~cm}^{-1} .{ }^{1} \mathbf{H}$ NMR (CDCl $\left.3,400 \mathbf{M H z}\right): \delta=0.27$ (s, 9H, a-H) $3.30(\mathrm{~s}, 1 \mathrm{H}, \mathrm{f}-\mathrm{H}), 7.24-7.30(\mathrm{~m}, 2 \mathrm{H},[\mathrm{c}+\mathrm{d}]-\mathrm{H}), 7.46-7.51(\mathrm{~m}, 2 \mathrm{H},[\mathrm{b}+\mathrm{e}]-\mathrm{H})$ ppm. ${ }^{13} \mathbf{C}$ NMR $\left(\mathbf{C D C l}_{3}, \mathbf{1 0 0} \mathrm{MHz}\right): \delta=80.0,81.1,99.0,103.1,125.0,126.1,128.1,128.4,132.0,132.4 \mathrm{ppm}$. 


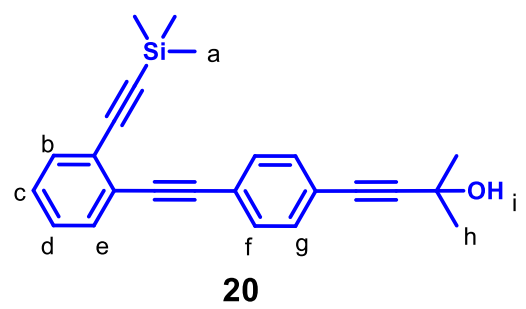

A $250 \mathrm{~mL}$ flask was charged with 19 (1.50 g, $7.56 \mathrm{mmol})$, 4-(4-bromophenyl)-2-methylbut-3-yn2-ol (1.44 g, $6.05 \mathrm{mmol})$, and CuI (72.0 mg, $378 \mu \mathrm{mol})$. Freshly distilled anhydrous $\mathrm{Et}_{3} \mathrm{~N}(65 \mathrm{~mL})$ was added and the solution was purged with $\mathrm{N}_{2}$ for $40 \mathrm{~min}$. After addition of $\mathrm{Pd}\left(\mathrm{PPh}_{3}\right)_{4}(436 \mathrm{mg}$, $377 \mu \mathrm{mol}$ ), the reaction mixture was heated to $80^{\circ} \mathrm{C}$ for $50 \mathrm{~h}$ (TLC). The reaction was allowed to cool to room temperature and the solvent was removed by rotary evaporation. The residue was dissolved in DCM $(50 \mathrm{~mL})$ and successively washed with deionized water $(50 \mathrm{~mL} \times 3)$ and saturated brine $(50 \mathrm{~mL})$. The organic layer was removed, dried over anhydrous $\mathrm{Na}_{2} \mathrm{SO}_{4}$ and evaporated. The chromatographic purification of the crude product on silica gel $(\phi=3.5 \mathrm{~cm}, l=$ $15 \mathrm{~cm})$ using $10 \%$ EtOAc in $n$-hexane $\left(R_{\mathrm{f}}=0.50, \mathrm{SiO}_{2}\right.$, EtOAc in $n$-hexane) furnished 20 as a yellow solid (2.10 g, 75\%). Mp: 60-62 ${ }^{\circ} \mathrm{C}$. IR (KBr): $\tilde{v}=504,561,642,759,842,958,1009$, 1155, 1247, 1368, 1439, 1505, 1935, 2148, 2971, 3056, $3451 \mathrm{~cm}^{-1} .{ }^{1} \mathbf{H}$ NMR (CDCl, 400 MHz): $\delta=0.24(\mathrm{~s}, 9 \mathrm{H}, \mathrm{a}-\mathrm{H}), 1.61(\mathrm{~s}, 6 \mathrm{H}, \mathrm{h}-\mathrm{H}), 2.01$ (bs, 1H, i-H), 7.24-7.28 (m, 2H, [c+d]-H), $7.38(\mathrm{~d}$, $\left.{ }^{3} J=8.8 \mathrm{~Hz}, 2 \mathrm{H}, \mathrm{g}-\mathrm{H}\right), 7.47-7.50(\mathrm{~m}, 4 \mathrm{H},[\mathrm{b}+\mathrm{e}+\mathrm{f}]-\mathrm{H}) \mathrm{ppm} .{ }^{13} \mathbf{C} \mathbf{~ N M R}\left(\mathbf{C D C l}_{3}, \mathbf{1 0 0} \mathbf{~ M H z}\right): \delta=0.0$, 31.4, 65.6, 81.8, 89.9, 92.9, 95.6, 98.7, 103.3, 122.7, 123.2, 125.7 (2C), 128.1, 128.2, 131.5 (2C), 131.7, 132.3 ppm. Elemental analysis: Calcd. for $\mathrm{C}_{24} \mathrm{H}_{24} \mathrm{OSi}$ : C, 80.85; H, 6.78; Found: C, 80.84; $\mathrm{H}, 6.88$.

((2-((4-Ethynylphenyl)ethynyl)phenyl)ethynyl)trimethylsilane (21)4

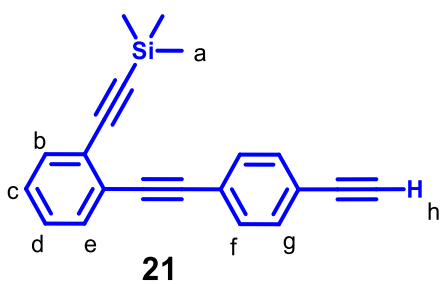

A solution of compound $20(1.10 \mathrm{~g}, 3.09 \mathrm{mmol})$ in anhydrous $\mathrm{PhMe}(40 \mathrm{~mL})$ was heated in an oil bath $\left(130^{\circ} \mathrm{C}\right)$. The reaction mixture was refluxed, then granular $\mathrm{NaOH}(135 \mathrm{mg}, 3.39 \mathrm{mmol})$ was 
added in one portion. The resultant mixture was stirred at $130^{\circ} \mathrm{C}$ for $1 \mathrm{~h}$. After completion (TLC), the reaction mixture was allowed to cool to room temperature. Then it was diluted with deionized water and extracted in PhMe $(25 \mathrm{~mL})$. The organic phase was washed with a saturated brine solution $(75 \mathrm{~mL})$ and dried over anhydrous $\mathrm{Na}_{2} \mathrm{SO}_{4}$. The solvent was removed in vacuo furnishing a brown oil that was purified by column chromatography on silica gel using $n$-hexane $\left(R_{\mathrm{f}}=0.15\right.$, $\mathrm{SiO}_{2}, n$-hexane). Purification yielded 21 as a bright yellow solid in 92\% yield (850 mg, 2.84 mmol). Mp: 55-56 ${ }^{\circ} \mathrm{C}$. IR (KBr): $\tilde{v}=463,503,603,660,752,846,948,1033,1092,1244,1318$, 1404, 1499, 1597, 1797, 1913, 2152, 2958, 3056, $3291 \mathrm{~cm}^{-1}$. ${ }^{\mathbf{1}} \mathbf{H}$ NMR (CDCl3, 400 MHz): $\delta=$ $0.27(\mathrm{~s}, 9 \mathrm{H}, \mathrm{a}-\mathrm{H}), 3.18(\mathrm{~s}, 1 \mathrm{H}, \mathrm{h}-\mathrm{H}), 7.27-7.30(\mathrm{~m}, 2 \mathrm{H},[\mathrm{c}+\mathrm{d}]-\mathrm{H}), 7.47-7.52(\mathrm{~m}, 6 \mathrm{H},[\mathrm{b}+\mathrm{e}+\mathrm{f}+\mathrm{g}]-$ H) ppm. ${ }^{13} \mathbf{C}$ NMR (CDCl3, $\left.100 \mathrm{MHz}\right): \delta=0.0,78.9,83.3,90.2,92.8,98.8,103.3,122.0,123.8$, 125.7 (2C), 128.1, 128.2, 131.5, 131.7, 132.0, 132.3 ppm. Elemental analysis: Calcd. for $\mathrm{C}_{21} \mathrm{H}_{18} \mathrm{Si}: \mathrm{C}, 84.51$; H, 6.08; Found: C, 84.89; H, 6.08.

3-((2-Iodophenyl)ethynyl)-2,9-dimesityl-1,10-phenanthroline (22)

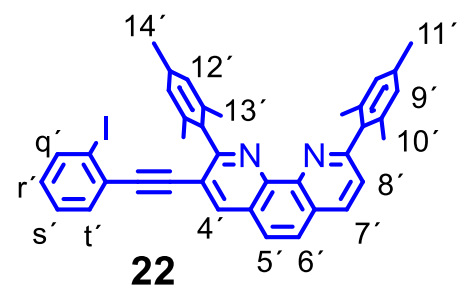

3-Ethynyl-2,9-dimesityl-1,10-phenanthroline (100 mg, $0.227 \mathrm{mmol})$ and 1,2-diiodobenzene (373 $\mathrm{mg}, 1.13 \mathrm{mmol}$ ) were placed in a sealed tube. It was evacuated and filled with $\mathrm{N}_{2}$, then freshly distilled $\mathrm{Et}_{3} \mathrm{~N}(20 \mathrm{~mL})$ and $\mathrm{DMF}(15 \mathrm{~mL})$ were added. The solution was degassed twice by using the freeze-pump-thaw method. $\mathrm{Pd}\left(\mathrm{PPh}_{3}\right)_{4}(26.3 \mathrm{mg}, 22.7 \mathrm{nmol})$ was added to this mixture that was stirred at $75{ }^{\circ} \mathrm{C}$ for $15 \mathrm{~h}$. After cooling to room temperature the solvents were removed under reduced pressure. The residue was extracted in DCM $(75 \mathrm{~mL})$ and washed with ice-cold water $(75$ $\mathrm{mL})$ and brine $(30 \mathrm{~mL})$. The organic layer was dried over anhydrous $\mathrm{Na}_{2} \mathrm{SO}_{4}$ and evaporated. The column chromatographic purification of the crude product on silica gel using $10 \%$ ethyl acetate in hexane $\left(R_{\mathrm{f}}=0.30, \mathrm{SiO}_{2}, 10 \%\right.$ ethyl acetate/hexane $)$ afforded compound $\mathbf{2 2}$ as colorless solid in 85\% yield (124 mg, $0.193 \mathrm{mmol})$. IR (KBr): $\tilde{v}=510,519,613,641,691,750,848,888,942$, 993, 1016, 1094, 1236, 1384, 1433, 1465, 1480, 1507, 1584, 1614, 2023, 2160, 2214, 2345, 2854, 
2915, 2951, 3002, $3050 \mathrm{~cm}^{-1}$. Mp. > $250{ }^{\circ} \mathrm{C} .{ }^{\mathbf{1}} \mathbf{H}$ NMR $\left(\mathbf{C D C l}_{3}, 400 \mathbf{M H z}\right): \delta=2.09$ (s, 6H, 10'-

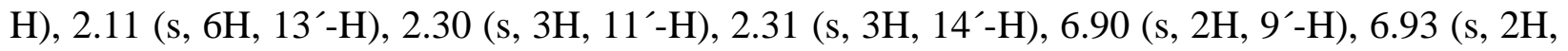
$\left.12^{\prime}-\mathrm{H}\right), 6.97\left(\mathrm{td},{ }^{3} J=8.0 \mathrm{~Hz},{ }^{4} J=1.8 \mathrm{~Hz}, \mathrm{~s}^{\prime}-\mathrm{H}\right), 7.13\left(\mathrm{dd},{ }^{3} J=8.0 \mathrm{~Hz},{ }^{4} J=1.8 \mathrm{~Hz}, \mathrm{q}^{\prime}-\mathrm{H}\right), 7.24(\mathrm{td}$, $\left.{ }^{3} J=8.0 \mathrm{~Hz},{ }^{4} J=1.8 \mathrm{~Hz}, \mathrm{r}^{\prime}-\mathrm{H}\right), 7.58\left(\mathrm{~d},{ }^{3} J=8.2 \mathrm{~Hz}, 1 \mathrm{H}, 8^{\prime}-\mathrm{H}\right), 7.79\left(\mathrm{dd},{ }^{3} J=8.0 \mathrm{~Hz},{ }^{4} J=1.8 \mathrm{~Hz}\right.$, $\left.\mathrm{t}^{\prime}-\mathrm{H}\right), 7.85\left(\mathrm{~d},{ }^{3} \mathrm{~J}=8.2 \mathrm{~Hz}, 1 \mathrm{H}, 6^{\prime}-\mathrm{H}\right), 7.88\left(\mathrm{~d},{ }^{3} \mathrm{~J}=8.2 \mathrm{~Hz}, 1 \mathrm{H}, 5^{\prime}-\mathrm{H}\right), 8.29$ (d, ${ }^{3} J=8.2 \mathrm{~Hz}, 1 \mathrm{H}, 7^{\prime}-$ $\mathrm{H}), 8.55$ (s, 1H, 4'-H) ppm. ${ }^{13} \mathbf{C}$ NMR (CDCl, $\left.100 \mathbf{M H z}\right): \delta=20.1,20.5,21.1,21.2,90.8,93.4$, 98.6, 120.1, 125.0, 125.1, 125.6, 125.7, 127.9, 128.0, 128.2, 128.5, 128.6, 128.7, 132.1, 132.3, 135.8, 136.1, 136.3, 136.8, 137.3, 137.5, 137.8, 138.9, 144.9, 145.9, 160.5, 161.9 ppm. Elemental analysis: Anal. Calcd for $\mathrm{C}_{38} \mathrm{H}_{31} \mathrm{IN}_{2}$ : C, 71.03; H, 4.86; N, 4.36. Found: C, 71.28; H, 4.57; N, 4.13. ESI-MS: $m / z(\%) 643.5(100)[\mathrm{M}+\mathrm{H}]^{+}$.

2,9-Dimethylsilyl)ethynyl)phenyl)ethynyl)phenyl)ethynyl)phenyl)ethynyl)-1,10-phenanthroline (23)

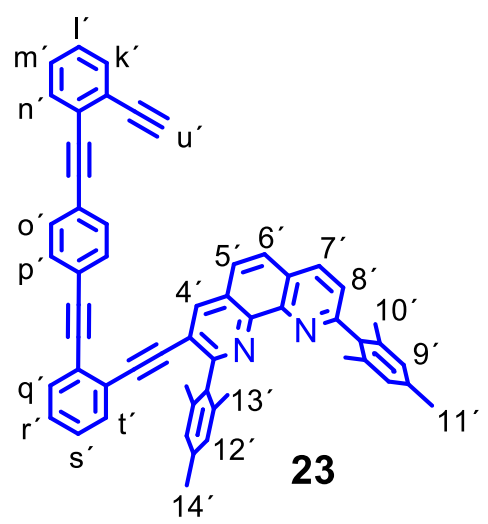

Compounds 21 (76.5 mg, $257 \mu \mathrm{mol})$ and $22(100 \mathrm{mg}, 171 \mu \mathrm{mol})$ were placed in a sealed tube. It was evacuated and filled with $\mathrm{N}_{2}$, then freshly distilled $\mathrm{Et}_{3} \mathrm{~N}(20 \mathrm{~mL})$ and DMF $(15 \mathrm{~mL})$ were added. The solution was degassed twice by using the freeze-pump-thaw method. To this mixture $\mathrm{Pd}\left(\mathrm{PPh}_{3}\right)_{4}(19.7 \mathrm{mg}, 25.7 \mu \mathrm{mol})$ was added and it was allowed to stir at $75^{\circ} \mathrm{C}$ for $15 \mathrm{~h}$. The mixture was cooled to room temperature and solvents were removed under reduced pressure. The residue was extracted in DCM $(75 \mathrm{~mL})$ and washed with ice-cold water $(75 \mathrm{~mL})$ and brine $(30 \mathrm{~mL})$. The organic layer was dried over anhydrous $\mathrm{Na}_{2} \mathrm{SO}_{4}$ and evaporated. The column chromatographic purification of the crude product on silica gel using $15 \%$ ethyl acetate in hexane $\left(R_{\mathrm{f}}=0.30, \mathrm{SiO}_{2}\right.$, $15 \%$ ethyl acetate/hexane) afforded a colorless solid compound. This compound was dissolved in 
$30 \mathrm{~mL}$ of THF and $30 \mathrm{~mL}$ of methanol. Aqueous solution $(30 \mathrm{~mL})$ of $\mathrm{KOH}(355 \mathrm{mg}, 2.58 \mathrm{mmol})$ was added to this mixture and stirred for $3 \mathrm{~h}$ at room temperature. The organic residue was extracted using dichloromethane and this solution was dried over anhydrous $\mathrm{Na}_{2} \mathrm{SO}_{4}$ and evaporated under vacuum. Finally the product was separated after passing through a small pad of neutral alumina using dichloromethane $\left(R_{\mathrm{f}}=0.40, \mathrm{SiO}_{2}, 100 \%\right.$ dichloromethane $)$ as eluent furnishing the pale yellow product in $75 \%$ yield $(95.0 \mathrm{mg}, 128 \mu \mathrm{mol})$. IR $(\mathbf{K B r}): \tilde{v}=508,543$, 559, 644, 757, 949, 1017, 1094, 1161, 1249, 1261, 1310, 1384, 1404, 1441, 1472, 1556, 1591, 1694, 1916, 2157, 2215, 2256, 2312, 2898, 2957, $3058 \mathrm{~cm}^{-1}$. Mp. $>250{ }^{\circ} \mathrm{C} .{ }^{1} \mathbf{H}$ NMR $\left(\mathbf{C D C l}_{3}\right.$,

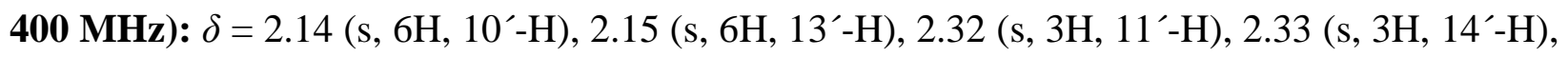
$3.41\left(\mathrm{~s}, 1 \mathrm{H}, \mathrm{u}^{\prime}-\mathrm{H}\right), 6.91\left(\mathrm{~s}, 2 \mathrm{H}, 9^{\prime}-\mathrm{H}\right), 6.93\left(\mathrm{~s}, 2 \mathrm{H}, 12^{\prime}-\mathrm{H}\right), 7.02\left(\mathrm{dd},{ }^{3} \mathrm{~J}=8.0 \mathrm{~Hz},{ }^{4} J=1.2 \mathrm{~Hz}, 1 \mathrm{H}\right.$, $\left.\mathrm{k}^{\prime}-\mathrm{H}\right), 7.23\left(\mathrm{td},{ }^{3} J=8.0 \mathrm{~Hz},{ }^{4} J=1.2 \mathrm{~Hz}, \mathrm{~m}^{\prime}-\mathrm{H}\right), 7.28\left(\mathrm{td},{ }^{3} J=8.0 \mathrm{~Hz},{ }^{4} J=1.6 \mathrm{~Hz}, 1^{\prime}-\mathrm{H}\right), 7.33(\mathrm{dd}$, $\left.{ }^{3} J=8.0 \mathrm{~Hz},{ }^{4} J=1.6 \mathrm{~Hz}, \mathrm{n}^{\prime}-\mathrm{H}\right), 7.36\left(\mathrm{td},{ }^{3} J=8.0 \mathrm{~Hz},{ }^{4} J=1.4 \mathrm{~Hz}, \mathrm{~s}^{\prime}-\mathrm{H}\right), 7.52\left(\mathrm{dd},{ }^{3} J=8.0 \mathrm{~Hz},{ }^{4} J\right.$ $\left.=1.6 \mathrm{~Hz}, \mathrm{q}^{\prime}-\mathrm{H}\right), 7.56-7.59\left(\mathrm{~m}, 3 \mathrm{H},\left[8^{\prime}+\mathrm{r}^{\prime}+\mathrm{t}^{\prime}\right]-\mathrm{H}\right), 7.62\left(\mathrm{bs}, 4 \mathrm{H},\left[\mathrm{o}^{\prime}+\mathrm{p}^{\prime}\right]-\mathrm{H}\right), 7.75\left(\mathrm{~d},{ }^{3} J=8.4 \mathrm{~Hz}\right.$, $\left.1 \mathrm{H}, 6^{\prime}-\mathrm{H}\right), 7.87\left(\mathrm{~d},{ }^{3} J=8.4 \mathrm{~Hz}, 1 \mathrm{H}, 5^{\prime}-\mathrm{H}\right), 8.27\left(\mathrm{~d},{ }^{3} J=8.4 \mathrm{~Hz}, 1 \mathrm{H}, 7^{\prime}-\mathrm{H}\right), 8.47$ (s, $\left.1 \mathrm{H}, 4^{\prime}-\mathrm{H}\right) \mathrm{ppm}$. ${ }^{13} \mathbf{C ~ N M R}\left(\mathbf{C D C l}_{3}, \mathbf{1 0 0} \mathbf{M H z}\right): \delta=20.2,20.6,21.1,21.2,71.3,82.1,89.9,90.2,91.3,93.1,93.2$, 93.4, 119.9, 123.3, 123.4, 124.7, 125.0, 125.2, 125.5, 125.6, 126.0, 126.8, 127.0, 127.5, 127.9, 128.2, 128.3, 128.5, 128.6, 131.6 (2C), 131.7 (2C), 131.8, 132.4, 132.7, 135.7, 136.2, 136.3, 136.9, 137.2, 137.5, 138.0, 139.0, 145.1, 146.0, 160.4, 161.7 ppm. Elemental analysis: Anal. Calcd for $\mathrm{C}_{56} \mathrm{H}_{40} \mathrm{~N}_{2} \bullet \mathrm{H}_{2} \mathrm{O}: \mathrm{C}, 88.62 ; \mathrm{H}, 5.58 ; \mathrm{N}, 3.69$. Found: C, 88.54; H, 5.62; N, 3.49. ESI-MS: $m / z(\%)$ $741.3(100)[\mathrm{M}+\mathrm{H}]^{+}$. 
Synthesis of nanoswitch 4

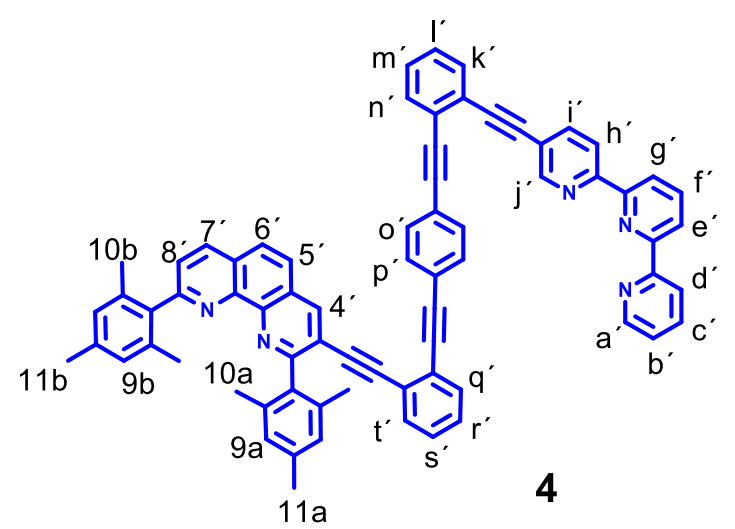

Compounds 23 (70.0 mg, $94.5 \mu \mathrm{mol}$ ) and 5-bromo-2,2':6',2"-terpyridine ( $88.5 \mathrm{mg}, 312 \mu \mathrm{mol})$ were placed in a sealed tube. It was evacuated and filled with $\mathrm{N}_{2}$, then freshly distilled $\mathrm{Et}_{3} \mathrm{~N}(20 \mathrm{~mL})$ and DMF $(15 \mathrm{~mL})$ were added. The solution was degassed twice by using the freeze-pump-thaw method. To this mixture $\mathrm{Pd}\left(\mathrm{PPh}_{3}\right)_{4}(10.9 \mathrm{mg}, 9.45 \mathrm{nmol})$ was added and it was allowed to stir at $90{ }^{\circ} \mathrm{C}$ for $15 \mathrm{~h}$. The mixture was cooled to room temperature, then the solvents were removed under reduced pressure. The residue was extracted in DCM $(75 \mathrm{~mL})$ and washed with ice-cold water $(75 \mathrm{~mL})$ and brine $(30 \mathrm{~mL})$. The organic layer was dried over anhydrous $\mathrm{Na}_{2} \mathrm{SO}_{4}$ and evaporated. The column chromatographic purification of the crude product on silica gel using $30 \%$ ethyl acetate in hexane ( $R_{\mathrm{f}}=0.30, \mathrm{SiO}_{2}, 30 \%$ ethyl acetate/hexane) afforded nanoswitch $\mathbf{4}$ as pale yellow solid in 70\% yield $(64.5 \mathrm{mg}, 66.2 \mu \mathrm{mol}) .{ }^{1} \mathbf{H}$ NMR $\left(\mathbf{C D}_{2} \mathbf{C l}_{2}, \mathbf{4 0 0} \mathbf{M H z}\right): \delta=2.01(\mathrm{~s}, 6 \mathrm{H}$, 10b-H), 2.05 (s, 6H, 10a-H), 2.32 (s, 3H, 11b-H), 2.35 (s, 3H, 11a-H), 6.93 (s, 2H, 9b-H), 6.95 (s, $2 \mathrm{H}, 9 \mathrm{a}-\mathrm{H}), 7.00\left(\mathrm{dd},{ }^{3} J=7.8 \mathrm{~Hz},{ }^{4} J=1.0 \mathrm{~Hz}, 1 \mathrm{H}, \mathrm{t}^{\prime}-\mathrm{H}\right), 7.23-7.34\left(\mathrm{~m}, 3 \mathrm{H},\left[\mathrm{b}^{\prime}+\mathrm{r}^{\prime}+\mathrm{s}^{\prime}\right]-\mathrm{H}\right), 7.39$ $7.46\left(\mathrm{~m}, 2 \mathrm{H},\left[\mathrm{m}^{\prime}+\mathrm{k}^{\prime}\right]-\mathrm{H}\right), 7.49\left(\mathrm{~d},{ }^{3} J=8.0 \mathrm{~Hz}, 1 \mathrm{H}, 8^{\prime}-\mathrm{H}\right), 7.54\left(\mathrm{dd},{ }^{3} J=7.8 \mathrm{~Hz},{ }^{4} J=1.0 \mathrm{~Hz}, 1 \mathrm{H}\right.$, $\left.\mathrm{q}^{\prime}-\mathrm{H}\right), 7.63-7.70\left(\mathrm{~m}, 6 \mathrm{H},\left[\mathrm{o}^{\prime}+\mathrm{p}^{\prime}+\mathrm{n}^{\prime}+\mathrm{l}^{\prime}\right]-\mathrm{H}\right), 7.74-7.79\left(\mathrm{~m}, 3 \mathrm{H},\left[\mathrm{c}^{\prime}+5^{\prime}+6^{\prime}\right]-\mathrm{H}\right), 7.89\left(\mathrm{t},{ }^{3} \mathrm{~J}=7.8 \mathrm{~Hz}\right.$, $\left.1 \mathrm{H}, \mathrm{f}^{\prime}-\mathrm{H}\right), 8.06\left(\mathrm{dd},{ }^{3} J=8.2 \mathrm{~Hz},{ }^{4} J=2.0 \mathrm{~Hz}, 1 \mathrm{H}, \mathrm{i}^{\prime}-\mathrm{H}\right), 8.19\left(\mathrm{~d},{ }^{3} J=8.0 \mathrm{~Hz}, 1 \mathrm{H}, 7^{\prime}-\mathrm{H}\right), 8.41-8.45$ (m, 2H, [g'+e']-H), $8.51\left(\mathrm{~s}, 1 \mathrm{H}, 4^{\prime}-\mathrm{H}\right), 8.53\left(\mathrm{ddd},{ }^{3} J=8.0 \mathrm{~Hz},{ }^{4} J=0.8 \mathrm{~Hz},{ }^{5} J=0.8 \mathrm{~Hz} 1 \mathrm{H}, \mathrm{d}^{\prime}-\mathrm{H}\right)$, $8.64\left(\mathrm{ddd},{ }^{3} J=4.8 \mathrm{~Hz},{ }^{4} J=1.8 \mathrm{~Hz},{ }^{5} J=0.8 \mathrm{~Hz}, 1 \mathrm{H}, \mathrm{a}^{\prime}-\mathrm{H}\right), 8.68\left(\mathrm{dd},{ }^{3} J=8.2 \mathrm{~Hz},{ }^{4} J=0.8 \mathrm{~Hz}, 1 \mathrm{H}\right.$, $\left.\mathrm{h}^{\prime}-\mathrm{H}\right), 8.91\left(\mathrm{dd},{ }^{4} J=2.0 \mathrm{~Hz},{ }^{5} \mathrm{~J}=0.8 \mathrm{~Hz}, 1 \mathrm{H}, \mathrm{j}^{\prime}-\mathrm{H}\right) \mathrm{ppm} .{ }^{13} \mathbf{C} \mathbf{N M R}\left(\mathbf{C D C l}_{3}, \mathbf{1 0 0} \mathbf{M H z}\right): \delta=20.1$, 20.5, 21.1, 21.2, 90.1, 90.3, 90.7, 91.2, 92.3, 93.1, 93.4, 93.5, 119.9, 120.3, 120.5, 121.1, 121.2, 121.3, 123.2, 123.5, 123.8, 125.0, 125.1, 125.2, 125.5, 125.6, 125.7, 126.8, 127.0, 127.5, 128.0, 128.2, 128.3, 128.4, 128.5, 128.6, 131.6, 131.7, 131.8, 132.0, 132.1, 132.5, 135.8, 136.1, 136.2, 
136.8, 137.3, 137.5, 137.8, 137.9, 138.9, 139.2, 144.9, 145.8, 149.1, 151.6, 154.5, 155.2, 155.5, 155.9, 160.4, 161.7 (2C) ppm. ESI-MS: $m / z(\%) 972.8(100)[\mathrm{M}+\mathrm{H}]^{+}$.

\section{Synthesis of other compounds}

4-(1-Benzyl-1H-1,2,3-triazol-4-yl)- $N, N$-dimethylaniline (7) ${ }^{5}$

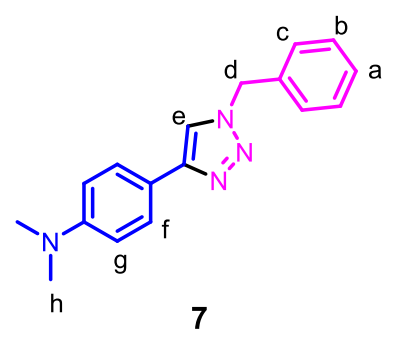

In a $100 \mathrm{~mL}$ flask 4-ethynyl- $N, N$-dimethylaniline $(60.0 \mathrm{mg}, 413 \mu \mathrm{mol})$ and benzyl azide $(55.0 \mathrm{mg}$, $413 \mu \mathrm{mol}$ ) were dissolved in $100 \mathrm{~mL}$ of $\mathrm{CH}_{2} \mathrm{Cl}_{2}: \mathrm{CHCl}_{3}$ (v/v 1:4). Tetrakis(acetonitrile)copper(I) tetrafluoroborate was added to this solution that was refluxed for $3 \mathrm{~h}$. After work-up, the organic phase was collected, dried over anhydrous $\mathrm{Na}_{2} \mathrm{SO}_{4}$ and filtered. The product was separated (91.8 $\mathrm{mg}, 330 \mu \mathrm{mol}, 80 \%$ ) by column chromatography over $\mathrm{SiO}_{2}$ using $1 \%$ ethyl acetate/dichloromethane as eluent $\left(R_{\mathrm{f}}=0.30, \mathrm{SiO}_{2}\right.$, in $1 \%$ ethyl acetate/dichloromethane). ${ }^{1} \mathbf{H} \mathbf{~ N M R}\left(\mathbf{C D C l}_{3}\right.$, $500 \mathrm{MHz}): \delta=2.97(\mathrm{~s}, 6 \mathrm{H}, \mathrm{h}-\mathrm{H}), 5.55(\mathrm{~s}, 2 \mathrm{H}, \mathrm{d}-\mathrm{H}), 6.74\left(\mathrm{~d},{ }^{3} J=8.0 \mathrm{~Hz}, 2 \mathrm{H}, \mathrm{g}-\mathrm{H}\right), 7.27-7.30(\mathrm{~m}$, 2H, b-H), 7.35-7.38 (m, 3H, [a+c]-H), $7.52(\mathrm{~s}, 1 \mathrm{H}, \mathrm{e}-\mathrm{H}), 7.66\left(\mathrm{~d},{ }^{3} \mathrm{~J}=8.0 \mathrm{~Hz}, 2 \mathrm{H}, \mathrm{f}-\mathrm{H}\right) \mathrm{ppm} .{ }^{13} \mathrm{C}$ NMR (CDCl 3,125 MHz): $\delta=40.4,54.1,112.4,118.0,118.8,126.6,128.0,128.6,129.1,135.0$, 148.7, 150.4 ppm. ESI-MS: $\mathrm{m} / z(\%) 279.4(100)[\mathrm{M}+\mathrm{H}]^{+}$. 


\subsection{Synthesis and characterization of metal complexes}

Pre-rotor complex ROT-1

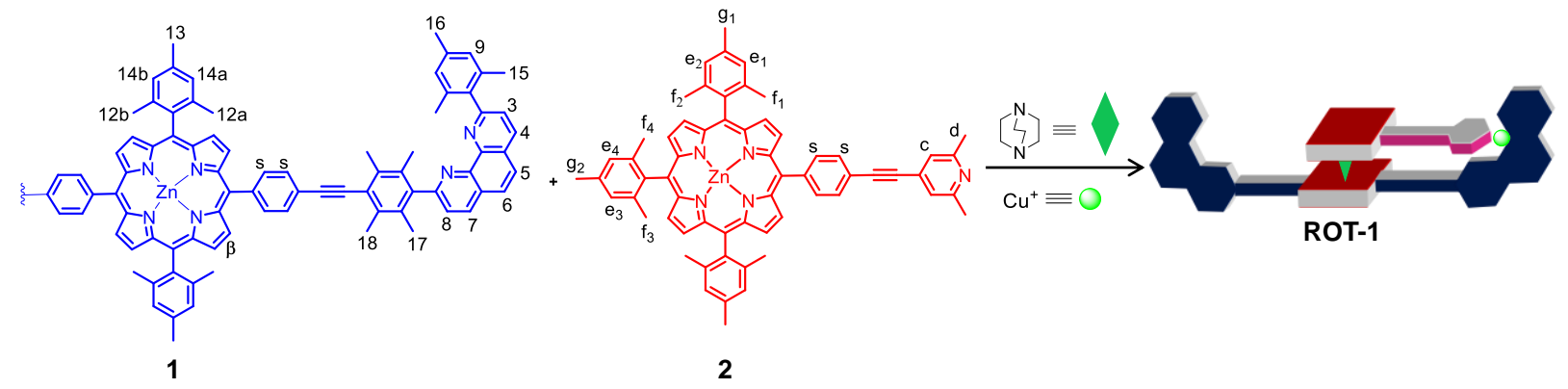

Protons at the complexed (c) vs. uncomplexed (u) phenanthroline site appear as a rule at different shifts.

In an NMR tube, stator $\mathbf{1}^{6}(2.09 \mathrm{mg}, 1.25 \mu \mathrm{mol})$ was mixed with 1 equiv of $\left[\mathrm{Cu}\left(\mathrm{CH}_{3} \mathrm{CN}_{4}\right] \mathrm{PF}_{6}\right.$ $(466 \mu \mathrm{g}, 1.25 \mu \mathrm{mol})$ and dissolved in $100 \mu \mathrm{L}$ of $\mathrm{CD}_{2} \mathrm{Cl}_{2}$, followed by addition of rotator 2 (1.17 $\mathrm{mg}, 1.25 \mu \mathrm{mol})$, DABCO $(145 \mu \mathrm{g}, 1.25 \mu \mathrm{mol})$ and additional $400 \mu \mathrm{L}$ of $\mathrm{CD}_{2} \mathrm{Cl}_{2}$. After subsequent sonication for 2 min the complex was obtained in quantitative yield. Mp: $>250{ }^{\circ} \mathrm{C}$. IR (KBr): $\tilde{v}$ $=558,721,797,845,995,1063,1203,1338,1380,1458,1492,1585,1609,1680,2222,2852$, 2920, $2951 \mathrm{~cm}^{-1} .{ }^{1} \mathbf{H}$ NMR (CD2 Cl2, $\left.400 \mathrm{MHz}\right): \delta=-[4.54-4.49]$ (m, 6H, $\mathrm{CH}_{2}$, DABCO), -[4.464.41] (m, 6H, $\left.\mathrm{CH}_{2}, \mathrm{DABCO}\right), 1.10$ (s, 6H, [f $\left.\left.\mathrm{f}_{1} / \mathrm{f}_{2}\right]-\mathrm{H}\right), 1.15$ (s, 6H, [f $\left.\left.\mathrm{f}_{2} / \mathrm{f}_{1}\right]-\mathrm{H}\right), 1.72$ (s, 6H, [12a/12b]H), 1.77 (s, 6H, [12b/12a]-H), 1.91 (s, 6H, [f $\left.\left.\mathrm{f}_{3}+\mathrm{f}_{4}\right]-\mathrm{H}\right), 2.06$ (s, 6H, 17u-H), 2.08 (s, 6H, d-H), 2.10 (s, 6H, 17c-H), 2.13 (s, 6H, 15u-H), 2.23 (s, 6H, 15c-H), 2.23 (s, 3H, 16u-H), 2.39 (s, 3H, 16c-H), 2.50 (s, 6H, 18u-H), 2.58-2.59 (s, 12H, [13+g $]-\mathrm{H}), 2.73$ (s, 3H, g2-H), 2.75 (s, 6H, 18c-H), 6.92 (s, 2H, 9u-H), 6.96 (bs, 2H, s-H), 7.00 (s, 2H, c-H), 7.02 (s, 2H, 9c-H), 7.08 (bs, 4H, [14a+14b]$\mathrm{H}), 7.19$ (s, 2H, [e $\left.\left.\mathrm{e}_{1} / \mathrm{e}_{2}\right]-\mathrm{H}\right), 7.26-7.29$ (m, 4H, [s+e $\left.\left.2 / \mathrm{e}_{1}\right]-\mathrm{H}\right), 7.33$ (bs, 2H, [e $\left.\left.3+\mathrm{e}_{4}\right]-\mathrm{H}\right), 7.47$ (bs, 2H, $\mathrm{s}-\mathrm{H}), 7.60\left(\mathrm{~d},{ }^{3} J=8.0 \mathrm{~Hz}, 1 \mathrm{H},[8 / 3] \mathrm{u}-\mathrm{H}\right), 7.63\left(\mathrm{~d},{ }^{3} J=8.0 \mathrm{~Hz}, 1 \mathrm{H},[3 / 8] \mathrm{u}-\mathrm{H}\right), 7.90\left(\mathrm{~d},{ }^{3} J=8.0 \mathrm{~Hz}\right.$, 4H, s-H), 7.94-7.97 (m, 5H, (5u+6u+[3/8]c+s)-H), $8.05\left(\mathrm{~d},{ }^{3} J=8.0 \mathrm{~Hz}, 1 \mathrm{H},[8 / 3] \mathrm{c}-\mathrm{H}\right), 8.25(\mathrm{~s}, 4 \mathrm{H}$, $\beta(\mathbf{1})-\mathrm{H}), 8.27$ (d, $\left.{ }^{3} J=4.4 \mathrm{~Hz}, 2 \mathrm{H}, \beta(\mathbf{1})-\mathrm{H}\right), 8.33$ (d, $\left.{ }^{3} J=4.4 \mathrm{~Hz}, 2 \mathrm{H}, \beta(\mathbf{1})-\mathrm{H}\right), 8.34$ (s, 2H, [5c+6c]H), $8.38\left(\mathrm{~d},{ }^{3} J=4.4 \mathrm{~Hz}, 2 \mathrm{H}, \beta(2)-\mathrm{H}\right), 8.39\left(\mathrm{~d},{ }^{3} J=8.0 \mathrm{~Hz}, 1 \mathrm{H},[7 / 4] \mathrm{u}-\mathrm{H}\right), 8.42\left(\mathrm{~d},{ }^{3} J=8.0 \mathrm{~Hz}\right.$, $1 \mathrm{H},[4 / 7] \mathrm{u}-\mathrm{H}), 8.52\left(\mathrm{~d},{ }^{3} J=4.4 \mathrm{~Hz}, 2 \mathrm{H}, \beta(2)-\mathrm{H}\right), 8.53\left(\mathrm{~d},{ }^{3} J=4.4 \mathrm{~Hz}, 2 \mathrm{H}, \beta(2)-\mathrm{H}\right), 8.57\left(\mathrm{~d},{ }^{3} J=4.6\right.$ $\mathrm{Hz}, 2 \mathrm{H}, \beta(2)-\mathrm{H}), 8.78\left(\mathrm{~d},{ }^{3} J=8.0 \mathrm{~Hz}, 1 \mathrm{H},[7 / 4] \mathrm{c}-\mathrm{H}\right), 8.79\left(\mathrm{~d},{ }^{3} J=8.0 \mathrm{~Hz}, 1 \mathrm{H},[4 / 7] \mathrm{c}-\mathrm{H}\right) \mathrm{ppm}$.

Elemental analysis: Anal. Calcd for $\mathrm{C}_{182} \mathrm{H}_{159} \mathrm{CuN}_{15} \mathrm{Zn}_{2} \cdot 2 \mathrm{CH}_{2} \mathrm{Cl}_{2}$ : C, 75.29; H, 5.54; N, 7.15. Found: C, 75.04; H, 5.60; N, 6.80. ESI-MS: $m / z(\%)=2662.6(100)[\mathrm{Cu}(\mathbf{1})(2)]^{+}$. 
Nanorotor ROT-2

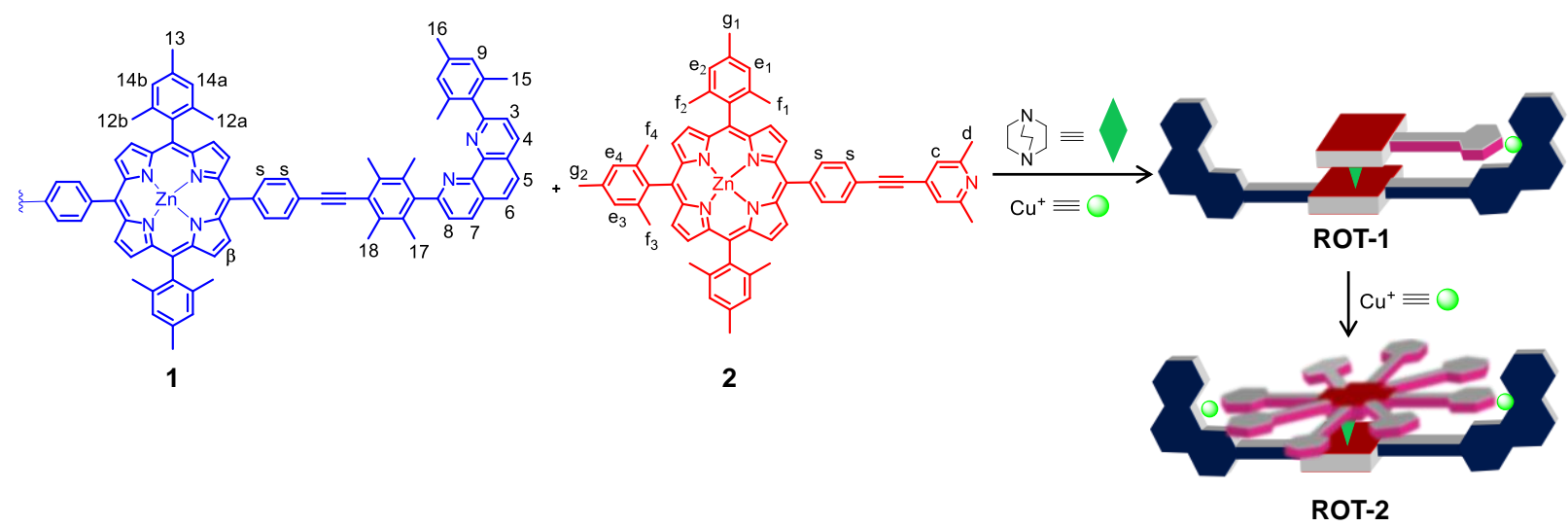

One equiv of $\left[\mathrm{Cu}\left(\mathrm{CH}_{3} \mathrm{CN}\right)_{4}\right] \mathrm{PF}_{6}(466 \mu \mathrm{g}, 1.25 \mu \mathrm{mol})$ was added to complex ROT-1 (3.87 mg, $1.25 \mu \mathrm{mol})$. Sonication for $2 \mathrm{~min}$ afforded ROT-2 quantitatively. Mp:. $>250^{\circ} \mathrm{C}$. IR (KBr): $\tilde{v}=$ 558, 726, 798, 847, 996, 1063, 1203, 1336, 1379, 1438, 1457, 1493, 1589, 1611, 2222, 2852, 2922 , $2954 \mathrm{~cm}^{-1} .{ }^{1} \mathbf{H}$ NMR (CD2 $\left.\mathbf{C l}_{2}, \mathbf{4 0 0} \mathbf{M H z}\right): \delta=-[4.51-4.47]$ (m, 6H, CH, $\left.\mathrm{DABCO}\right),-[4.44-4.40]$ (m, 6H, CH, DABCO), 1.09 (s, 6H, [f $\left.\left.\mathrm{f}_{1} / \mathrm{f}_{2}\right]-\mathrm{H}\right), 1.13$ (s, 6H, [f $\left.\left.\mathrm{f}_{2} / \mathrm{f}_{1}\right]-\mathrm{H}\right), 1.45\left(\mathrm{~s}, 3 \mathrm{H},\left[\mathrm{f}_{3} / \mathrm{f}_{4}\right]-\mathrm{H}\right), 1.55$ (s, 3H, [f $\left.\left.\mathrm{f}_{4} / \mathrm{f}_{3}\right]-\mathrm{H}\right), 1.72(\mathrm{~s}, 6 \mathrm{H},[12 \mathrm{a} / 12 \mathrm{~b}]-\mathrm{H}), 1.91$ (s, 6H, [12b/12a]-H), 2.05 (s, 6H, d-H), 2.11 (bs, 12H, 15-H), 2.22 (s, 12H, 17-H), 2.37 (s, 6H, 16-H), 2.49 (s, 6H, 13-H), 2.58 (s, 12H, 18-H), 2.71 (s, 3H, g $2-\mathrm{H}), 2.75$ (s, 6H, g $1-\mathrm{H}), 6.93(\mathrm{~s}, 2 \mathrm{H}, \mathrm{s}-\mathrm{H}), 7.04-7.10$ (m, 6H, [14a+14b+e $\left.\left.1 / \mathrm{e}_{2}\right]-\mathrm{H}\right), 7.23$ (s, 2H, [e $\left.\left.2 / \mathrm{e}_{1}\right]-\mathrm{H}\right), 7.24$ (s, 2H, s-H), 7.27 (s, 4H, 9-H), 7.31-7.35 (m, 4H, [c+e $\left.\left.\mathrm{e}_{3}+\mathrm{e}_{4}\right]-\mathrm{H}\right), 7.44$ (bs, 2H, s-H), 7.87-7.96 (m, 6H, [s+3/8]-H), $8.01\left(\mathrm{~d},{ }^{3} J=8.0 \mathrm{~Hz}, 2 \mathrm{H},[8 / 3]-\mathrm{H}\right), 8.18-8.23$ (s, 6H, $[5+6+\mathrm{s}]-\mathrm{H}), 8.28\left(\mathrm{~d}, 4 \mathrm{H},{ }^{3} J=4.6 \mathrm{~Hz}, \beta(\mathbf{1})-\mathrm{H}\right), 8.31\left(\mathrm{~d},{ }^{3} \mathrm{~J}=4.6 \mathrm{~Hz}, 2 \mathrm{H}, \beta(\mathbf{1})-\mathrm{H}\right), 8.34-8.38(\mathrm{~m}$, $6 \mathrm{H}, \beta(\mathbf{1})+\beta(\mathbf{2})-\mathrm{H}), 8.50$ (bs, $2 \mathrm{H}, \beta(\mathbf{2})-\mathrm{H}), 8.54\left(\mathrm{~d},{ }^{3} J=4.6 \mathrm{~Hz}, 2 \mathrm{H}, \beta(\mathbf{2})-\mathrm{H}\right), 8.77\left(\mathrm{~d},{ }^{3} J=8.0 \mathrm{~Hz}\right.$, $2 \mathrm{H},[4 / 7]-\mathrm{H}), 8.78\left(\mathrm{~d},{ }^{3} \mathrm{~J}=8.0 \mathrm{~Hz}, 4 \mathrm{H},[7 / 4]-\mathrm{H}\right) \mathrm{ppm}$. ESI-MS: $m / z(\%)=1363.2(100)$ $\left[\mathrm{Cu}_{2}(\mathbf{1})(\mathbf{2})\right]^{2+}, 1829.5(15)\left[\mathrm{Cu}_{2}(\mathbf{1})(\mathbf{2})_{2}\right]^{2+}$. 
Synthesis of $[\mathrm{Cu}(\mathbf{3})]^{+}$

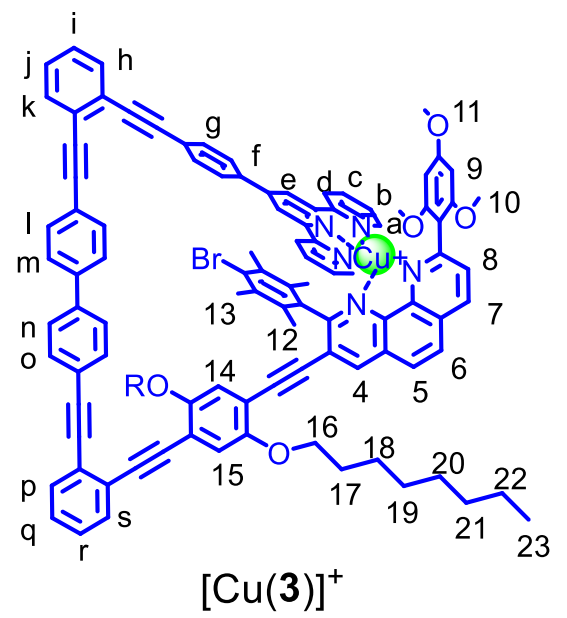

In an NMR tube, compound $3(725 \mu \mathrm{g}, 447 \mathrm{nmol})$ was dissolved in $500 \mathrm{~mL}$ of $\mathrm{CD}_{2} \mathrm{Cl}_{2}$, then $\left[\mathrm{Cu}\left(\mathrm{CH}_{3} \mathrm{CN}\right)_{4}\right] \mathrm{PF}_{6}(167 \mu \mathrm{g}, 447 \mathrm{nmol})$ was added. Immediately the solution assumed a reddishbrown color indicating the formation of the metal complex. The complex was unambiguously characterized by ${ }^{1} \mathrm{H}$ NMR, ${ }^{1} \mathrm{H}-{ }^{1} \mathrm{H}$ COSY, ESI-MS and elemental analysis. IR (KBr): $\tilde{v}=557$, 690, 757, 792, 844, 1004, 1021, 1127, 1157, 1206, 1222, 1280, 1338, 1384, 1427, 1467, 1499 , 1587, 1610, 2027, 2142, 2204, 2347, 2856, 2927, $2954 \mathrm{~cm}^{-1}$. Mp. $>250{ }^{\circ} \mathrm{C} .{ }^{1} \mathbf{H}$ NMR $\left(\mathbf{C D}_{2} \mathbf{C l}_{2}\right.$, $400 \mathrm{MHz}): \delta=0.76\left(\mathrm{t},{ }^{3} J=7.2 \mathrm{~Hz}, 3 \mathrm{H},\left[23 / 23^{\prime}\right]-\mathrm{H}\right), 0.82\left(\mathrm{t},{ }^{3} J=7.2 \mathrm{~Hz}, 3 \mathrm{H},\left[23^{\prime} / 23\right]-\mathrm{H}\right), 1.05-$ $1.08\left(\mathrm{~m}, 4 \mathrm{H},\left[22+22^{\prime}\right]-\mathrm{H}\right), 1.20-1.29\left(\mathrm{~m}, 16 \mathrm{H},\left[18+19+20+21+18^{\prime}+19^{\prime}+20^{\prime}+21^{\prime}\right]-\mathrm{H}\right), 1.40(\mathrm{~s}, 6 \mathrm{H}$, 13-H), $1.47\left(\mathrm{t},{ }^{3} J=7.2 \mathrm{~Hz}, 2 \mathrm{H},\left[17 / 17^{\prime}\right]-\mathrm{H}\right), 1.60\left(\mathrm{t},{ }^{3} J=7.2 \mathrm{~Hz}, 2 \mathrm{H},\left[17^{\prime} / 17\right]-\mathrm{H}\right), 1.89$ (s, 6H, $12-$ H), $3.27(\mathrm{~s}, 12 \mathrm{H}, 10-\mathrm{H}), 3.58(\mathrm{~s}, 6 \mathrm{H}, 11-\mathrm{H}), 3.66\left(\mathrm{t},{ }^{3} J=7.2 \mathrm{~Hz}, 2 \mathrm{H},\left[16 / 16^{\prime}\right]-\mathrm{H}\right), 3.82\left(\mathrm{t},{ }^{3} J=7.2\right.$ $\left.\mathrm{Hz}, 2 \mathrm{H},\left[16^{\prime} / 16\right]-\mathrm{H}\right), 5.72$ (s, 2H, 9-H), 6.11 (s, 1H, 14-H), 6.97 (s, 1H, 15-H), 7.15 (ddd, ${ }^{3} J=7.8$ $\left.\mathrm{Hz},{ }^{3} J=4.8 \mathrm{~Hz},{ }^{4} J=1.2 \mathrm{~Hz}, 1 \mathrm{H}, \mathrm{b}-\mathrm{H}\right), 7.32-7.38(\mathrm{~m}, 2 \mathrm{H},[\mathrm{i}+\mathrm{j}]-\mathrm{H}), 7.39\left(\mathrm{td},{ }^{3} J=7.8 \mathrm{~Hz},{ }^{4} J=1.2\right.$ $\mathrm{Hz}, 2 \mathrm{H}, \mathrm{c}-\mathrm{H}), 7.41-7.44(\mathrm{~m}, 2 \mathrm{H},[\mathrm{q}+\mathrm{r}]-\mathrm{H}), 7.54\left(\mathrm{dd},{ }^{3} J=8.0 \mathrm{~Hz},{ }^{4} J=1.6 \mathrm{~Hz}, 1 \mathrm{H}, \mathrm{k}-\mathrm{H}\right), 7.58(\mathrm{dd}$, $\left.{ }^{3} J=8.0 \mathrm{~Hz},{ }^{4} J=1.6 \mathrm{~Hz}, 1 \mathrm{H}, \mathrm{h}-\mathrm{H}\right), 7.59-7.61(\mathrm{~m}, 4 \mathrm{H},[\mathrm{n}+\mathrm{o}]-\mathrm{H}), 7.62-7.64(\mathrm{~m}, 3 \mathrm{H},[\mathrm{m}+\mathrm{p}]-\mathrm{H})$, 7.66-7.69 (m, 3H, [l+s]-H), $7.92\left(\mathrm{~d},{ }^{3} J=8.0 \mathrm{~Hz}, 2 \mathrm{H}, \mathrm{g}-\mathrm{H}\right), 7.95\left(\mathrm{~d},{ }^{3} J=8.0 \mathrm{~Hz}, 1 \mathrm{H}, 8-\mathrm{H}\right), 8.04(\mathrm{~d}$, $\left.{ }^{3} J=8.0 \mathrm{~Hz}, 2 \mathrm{H}, \mathrm{f}-\mathrm{H}\right), 8.06\left(\mathrm{~d},{ }^{3} \mathrm{~J}=8.0 \mathrm{~Hz}, 1 \mathrm{H},[5 / 6]-\mathrm{H}\right), 8.12\left(\mathrm{ddd},{ }^{3} \mathrm{~J}=4.8 \mathrm{~Hz},{ }^{4} J=1.2 \mathrm{~Hz},{ }^{5} \mathrm{~J}=\right.$ $0.8 \mathrm{~Hz}, 2 \mathrm{H}, \mathrm{a}-\mathrm{H}), 8.20\left(\mathrm{~d},{ }^{3} \mathrm{~J}=8.0 \mathrm{~Hz}, 1 \mathrm{H},[6 / 5]-\mathrm{H}\right), 8.25\left(\mathrm{ddd},{ }^{3} J=7.8 \mathrm{~Hz},{ }^{4} J=1.2 \mathrm{~Hz},{ }^{5} \mathrm{~J}=0.8\right.$ $\mathrm{Hz}, 2 \mathrm{H}, \mathrm{d}-\mathrm{H}), 8.39$ (s, 2H, e-H), $8.58(\mathrm{~s}, 1 \mathrm{H}, 4-\mathrm{H}), 8.66\left(\mathrm{~d},{ }^{3} J=8.0 \mathrm{~Hz}, 1 \mathrm{H}, 7-\mathrm{H}\right) \mathrm{ppm}$. Elemental analysis: Anal. Calcd for $\mathrm{C}_{108} \mathrm{H}_{94} \mathrm{BrCuF}_{6} \mathrm{~N}_{5} \mathrm{O}_{5 \mathrm{P}} 4 \mathrm{CH}_{2} \mathrm{Cl}_{2} \bullet \mathrm{H}_{2} \mathrm{O}: \mathrm{C}, 66.05 ; \mathrm{H}, 4.94 ; \mathrm{N}, 3.50$. Found: C, 65.88; H, 4.97; N, 3.25. ESI-MS: $m / z$ (\%) $1684.5(100)[\mathrm{Cu}(3)]^{+}$. 
Synthesis of $[\mathbf{Z n}(3)]^{2+}$

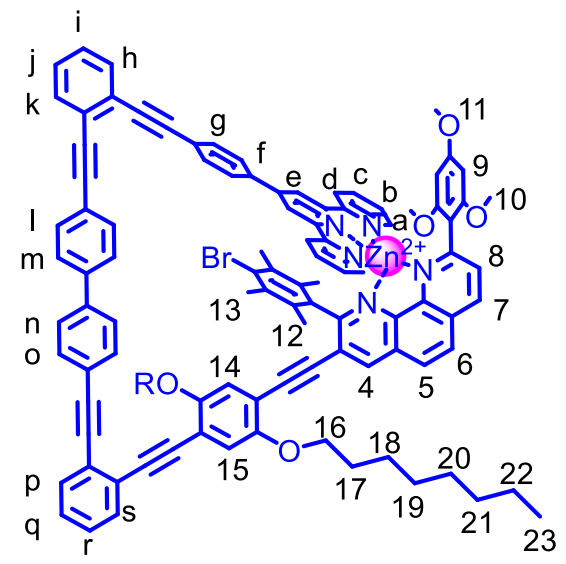

In an NMR tube, compound $3(754 \mu \mathrm{g}, 464 \mathrm{nmol})$ was dissolved in $500 \mathrm{~mL}$ of $\mathrm{CD}_{2} \mathrm{Cl}_{2}$. A solution of $\mathrm{Zn}(\mathrm{OTf})_{2}(169 \mu \mathrm{g}, 447 \mathrm{nmol})$ in $\mathrm{CD}_{3} \mathrm{CN}(7 \mu \mathrm{L})$ was added to it. The complex was then unambiguously characterized by ${ }^{1} \mathrm{H}$ NMR, ${ }^{1} \mathrm{H}-{ }^{1} \mathrm{H}$ COSY, ESI-MS and elemental analysis. IR $(\mathrm{KBr}): \tilde{v}=639,761,1031,1163,1227,1256,1384,1476,1604,1715,2060,2150,2224,2320$, 2864, 2929, $2940 \mathrm{~cm}^{-1}$. Mp. > $250{ }^{\circ} \mathrm{C} .{ }^{1} \mathbf{H}$ NMR $\left(\mathbf{C D}_{2} \mathbf{C l}_{2}, 400 \mathrm{MHz}\right): \delta=0.75\left(\mathrm{t},{ }^{3} \mathrm{~J}=7.2 \mathrm{~Hz}\right.$, $\left.3 \mathrm{H},\left[23 / 23^{\prime}\right]-\mathrm{H}\right), 0.82$ (t, $\left.{ }^{3} J=7.2 \mathrm{~Hz}, 3 \mathrm{H},\left[23^{\prime} / 23\right]-\mathrm{H}\right), 0.96$ (s, 6H, 13-H), 1.05-1.11 (m, 8H, $\left.\left[21+21^{\prime}+22+22^{\prime}\right]-\mathrm{H}\right), 1.17-1.25\left(\mathrm{~m}, 12 \mathrm{H},\left[18+19+20+18^{\prime}+19^{\prime}+20^{\prime}\right]-\mathrm{H}\right), 1.45\left(\mathrm{t},{ }^{3} \mathrm{~J}=7.2 \mathrm{~Hz}, 2 \mathrm{H}\right.$, [17/17']-H), 1.54 (merged with $\mathrm{H}_{2} \mathrm{O}$ signal, 2H, [17'/17]-H), 1.89 (s, 6H, 12-H), 3.27 (s, 6H, 10H), 3.58 (s, 3H, 11-H), 3.66 (t, $\left.{ }^{3} J=7.2 \mathrm{~Hz}, 2 \mathrm{H},\left[16 / 16^{\prime}\right]-\mathrm{H}\right), 3.82\left(\mathrm{t},{ }^{3} J=7.2 \mathrm{~Hz}, 2 \mathrm{H},\left[16^{\prime} / 16\right]-\mathrm{H}\right)$, $5.72(\mathrm{~s}, 2 \mathrm{H}, 9-\mathrm{H}), 6.11(\mathrm{~s}, 1 \mathrm{H}, 14-\mathrm{H}), 6.97(\mathrm{~s}, 1 \mathrm{H}, 15-\mathrm{H}), 7.32-7.35(\mathrm{~m}, 2 \mathrm{H},[\mathrm{i}+\mathrm{j}]-\mathrm{H}), 7.42-7.45$ $(\mathrm{m}, 2 \mathrm{H},[\mathrm{q}+\mathrm{r}]-\mathrm{H}), 7.53\left(\mathrm{dd},{ }^{3} J=8.0 \mathrm{~Hz},{ }^{4} J=1.6 \mathrm{~Hz}, 1 \mathrm{H}, \mathrm{k}-\mathrm{H}\right), 7.59-7.61(\mathrm{~m}, 7 \mathrm{H},[\mathrm{b}+\mathrm{n}+\mathrm{o}+\mathrm{h}]-\mathrm{H})$, 7.62-7.64 (m, 3H, [m+p]-H), 7.66-7.69 (m, 3H, [1+s]-H), $7.76\left(\mathrm{ddd},{ }^{3} J=4.8 \mathrm{~Hz},{ }^{4} J=1.2 \mathrm{~Hz},{ }^{5} J\right.$ $=0.8 \mathrm{~Hz}, 2 \mathrm{H}, \mathrm{a}-\mathrm{H}), 7.99\left(\mathrm{~d},{ }^{3} \mathrm{~J}=8.4 \mathrm{~Hz}, 2 \mathrm{H}, \mathrm{g}-\mathrm{H}\right), 8.13\left({ }^{3} \mathrm{~J}=8.4 \mathrm{~Hz}, 1 \mathrm{H}, 8-\mathrm{H}\right), 8.25\left(\mathrm{~d},{ }^{3} \mathrm{~J}=8.4\right.$ $\mathrm{Hz}, 2 \mathrm{H}, \mathrm{f}-\mathrm{H}), 8.35\left(\mathrm{td},{ }^{3} \mathrm{~J}=8.0 \mathrm{~Hz},{ }^{4} \mathrm{~J}=1.2 \mathrm{~Hz}, \mathrm{c}-\mathrm{H}\right), 8.39\left(\mathrm{~d},{ }^{3} \mathrm{~J}=8.0 \mathrm{~Hz},[5 / 6]-\mathrm{H}\right), 8.52\left(\mathrm{~d},{ }^{3} \mathrm{~J}=\right.$ $8.0 \mathrm{~Hz},[6 / 5]-\mathrm{H}), 8.72(\mathrm{~s}, 2 \mathrm{H}, \mathrm{e}-\mathrm{H}), 8.78\left(\mathrm{ddd},{ }^{3} J=8.0 \mathrm{~Hz},{ }^{4} J=0.8 \mathrm{~Hz},{ }^{5} J=0.8 \mathrm{~Hz}, 2 \mathrm{H}, \mathrm{d}-\mathrm{H}\right), 8.93$ (s, $1 \mathrm{H}, 4-\mathrm{H}), 9.02\left(\mathrm{~d},{ }^{3} \mathrm{~J}=8.4 \mathrm{~Hz}, 2 \mathrm{H}, 7-\mathrm{H}\right) \mathrm{ppm}$. Elemental analysis: Anal. Calcd for $\mathrm{C}_{110} \mathrm{H}_{94} \mathrm{BrF}_{6} \mathrm{~N}_{5} \mathrm{O}_{11} \mathrm{~S}_{2} \mathrm{Zn}_{2} \cdot 1.5 \mathrm{CH}_{2} \mathrm{Cl}_{2}: \mathrm{C}, 63.39 ; \mathrm{H}, 4.63 ; \mathrm{N}, 3.31$. Found: C, 63.36; H, 4.74; N, 2.91. ESI-MS: $m / z(\%) 843.1(100)[\mathrm{Zn}(3)]^{2+}$. 
Synthesis of $[\mathrm{Cu}(\mathbf{4})]^{+}$

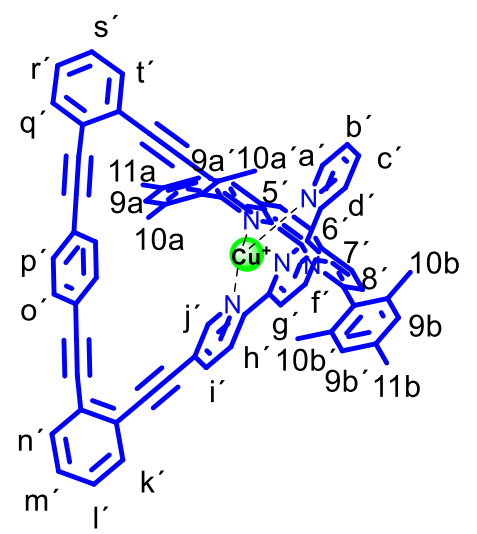

In an NMR tube, compound $4(423 \mu \mathrm{g}, 435 \mathrm{nmol})$ was dissolved in $500 \mathrm{~mL}$ of $\mathrm{CD}_{2} \mathrm{Cl}_{2}$, then $\left[\mathrm{Cu}\left(\mathrm{CH}_{3} \mathrm{CN}\right)_{4}\right] \mathrm{PF}_{6}(162 \mu \mathrm{g}, 435 \mathrm{nmol})$ was added. Immediately, the solution assumed a reddishbrown color, indicating the formation of the metal complex. The complex was then unambiguously characterized by ${ }^{1} \mathrm{H}$ NMR, ${ }^{1} \mathrm{H}-{ }^{1} \mathrm{H}$ COSY, ESI-Ms and elemental analysis. Mp. $>250{ }^{\circ} \mathrm{C}$. IR (KBr): $\tilde{v}=557,587,610,633,666.1,695,722,755,781,843,868,897,923,953,986,1015$, 1039, 1042, 1073, 1200, 1240, 1263, 1299, 1329, 1384, 1451, 1510.7, 1603, 1636, 1725, 2223, 2224, 2230, 2235, 2856, 2922, $2952 \mathrm{~cm}^{-1} .{ }^{1} \mathbf{H}$ NMR ( $\left.\mathbf{C D}_{2} \mathbf{C l}_{2}, 400 \mathbf{M H z}\right): \delta=1.32(\mathrm{~s}, 3 \mathrm{H}$, [10b/10b']-H), 1.49 (s, 3H, [10b'/10b]-H), 1.78 (s, 3H, [10a/10a']-H), 1.79 (s, 3H, [10a'/10a]-H), 1.92 (s, 3H, 11b-H), 1.99 (s, 3H, 11a-H), 6.12 (s, 1H, [9b/9b']-H), 6.23 (s, 1H, [9b'/9b]-H), 6.34

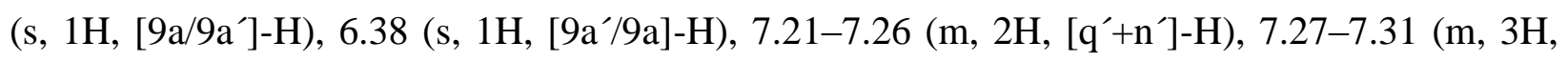
$\left.\left[\mathrm{r}^{\prime}+\mathrm{m}+\mathrm{c}^{\prime}\right]-\mathrm{H}\right), 7.32-7.38\left(\mathrm{~m}, 3 \mathrm{H},\left[\mathrm{g}^{\prime}+\mathrm{h}^{\prime}+\mathrm{b}^{\prime}\right]-\mathrm{H}\right), 7.39-7.43\left(\mathrm{~m}, 4 \mathrm{H},\left[\mathrm{o}^{\prime}+\mathrm{p}^{\prime}\right]-\mathrm{H}\right), 7.44-7.49(\mathrm{~m}, 2 \mathrm{H}$, $\left.\left[\mathrm{l}^{\prime}+\mathrm{s}^{\prime}\right]-\mathrm{H}\right), 7.56-7.61\left(\mathrm{~m}, 2 \mathrm{H},\left[\mathrm{t}^{\prime}+\mathrm{k}^{\prime}\right]-\mathrm{H}\right), 7.69\left(\mathrm{ddd},{ }^{3} J=8.0 \mathrm{~Hz},{ }^{3} J=1.8 \mathrm{~Hz},{ }^{5} J=0.8 \mathrm{~Hz}, 1 \mathrm{H}, \mathrm{d}^{\prime}-\right.$ H), $7.76\left(\mathrm{~d},{ }^{3} J=8.2 \mathrm{~Hz}, 1 \mathrm{H}, 8^{\prime}-\mathrm{H}\right), 7.78\left(\mathrm{~d},{ }^{3} J=8.2 \mathrm{~Hz}, 1 \mathrm{H}, 6^{\prime}-\mathrm{H}\right), 7.88-7.94\left(\mathrm{~m}, 3 \mathrm{H},\left[\mathrm{e}^{\prime}+\mathrm{j}^{\prime}+\mathrm{f}^{\prime}\right]-\right.$ H), $8.02-8.08\left(\mathrm{~m}, 2 \mathrm{H},\left[\mathrm{a}^{\prime}+\mathrm{i}^{\prime}\right]-\mathrm{H}\right), 8.12\left(\mathrm{~d},{ }^{3} J=8.2 \mathrm{~Hz}, 1 \mathrm{H}, 5^{\prime}-\mathrm{H}\right), 8.51\left(\mathrm{~s}, 1 \mathrm{H}, 4^{\prime}-\mathrm{H}\right), 8.56\left(\mathrm{~d},{ }^{3} J=\right.$ $\left.8.2 \mathrm{~Hz}, 1 \mathrm{H}, 7^{\prime}-\mathrm{H}\right) \mathrm{ppm}$. ESI-MS: $m / z(\%) 1034.9(100)[\mathrm{Cu}(\mathbf{4})]^{+}$. 
Synthesis of $[\operatorname{Hg}(\mathbf{4})]^{2+}$

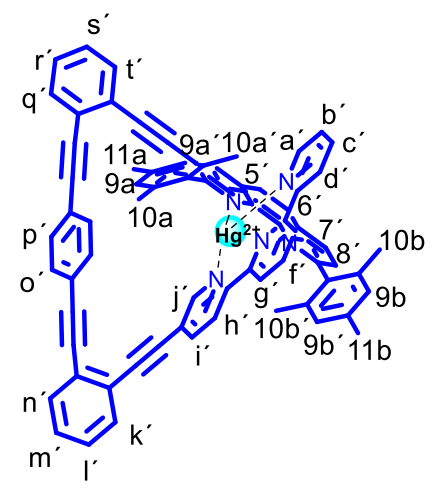

In an NMR tube, compound $4(423 \mu \mathrm{g}, 435 \mathrm{nmol})$ was dissolved in $500 \mathrm{~mL}$ of $\mathrm{CD}_{2} \mathrm{Cl}_{2}$, then a solution of $\mathrm{HgClO}_{4}(197 \mu \mathrm{g}, 435 \mathrm{nmol})$ in $\mathrm{CD}_{3} \mathrm{CN}$ was added. The complex was then unambiguously characterized by ${ }^{1} \mathrm{H}$ NMR, ${ }^{1} \mathrm{H}^{-1} \mathrm{H}$ COSY, ESI-MS and elemental analysis. Mp. $>250{ }^{\circ} \mathrm{C}$. IR (KBr): $\tilde{v}=501,523,560,573,610.0,639,668,702,761,804,824,847,867,1033.0,1065$, 1108, 1161, 1230, 1259, 1385, 1451, 1484, 1510, 1563, 1632, 2220, 2215, 2230, 2235, 2853, 2922 , $2959 \mathrm{~cm}^{-1} .{ }^{1} \mathbf{H}$ NMR ( $\mathbf{C D}_{2} \mathbf{C l}_{2}, 400$ MHz): $\delta=1.17$ (s, 3H, [10b/10b']-H), 1.47 (s, 3H, [10b'/10b]H), 1.52 (s, 3H, [10a/10a']-H), 1.69 (s, 3H, [10a/10a'+11b)-H), 1.78 (s, 3H, 11a-H), 6.06 (s, 1H, [9b/9b']-H), 6.12 (s, 1H, [9b'/9b]-H), 6.20 (s, 1H, [9a/9a']-H), 6.28 (s, 1H, [9a'/9a]-H), 7.20 (d, $\left.{ }^{3} J=8.4 \mathrm{~Hz}, 2 \mathrm{H},\left[\mathrm{o}^{\prime} / \mathrm{p}^{\prime}\right]-\mathrm{H}\right), 7.26\left(\mathrm{~d},{ }^{3} J=8.4 \mathrm{~Hz}, 2 \mathrm{H},\left[\mathrm{p}^{\prime} / \mathrm{o}^{\prime}\right]-\mathrm{H}\right), 7.31\left(\mathrm{td},{ }^{3} \mathrm{~J}=8.0 \mathrm{~Hz},{ }^{4} J=1.6 \mathrm{~Hz}\right.$, $\left.1^{\prime}-\mathrm{H}\right), 7.37\left(\mathrm{td},{ }^{3} J=8.0 \mathrm{~Hz},{ }^{4} J=1.6 \mathrm{~Hz}, \mathrm{~s}^{\prime}-\mathrm{H}\right), 7.38-7.46\left(\mathrm{~m}, 5 \mathrm{H},\left[\mathrm{a}^{\prime}+\mathrm{q}^{\prime}+\mathrm{m}^{\prime}+\mathrm{n}^{\prime}+\mathrm{r}^{\prime}\right]-\mathrm{H}\right), 7.53(\mathrm{ddd}$, $\left.{ }^{3} J=8.0 \mathrm{~Hz},{ }^{4} J=1.6 \mathrm{~Hz},{ }^{5} \mathrm{~J}=0.8 \mathrm{~Hz}, 1 \mathrm{H}, \mathrm{k}^{\prime}-\mathrm{H}\right), 7.62\left(\mathrm{ddd},{ }^{3} J=8.0 \mathrm{~Hz},{ }^{4} J=1.6 \mathrm{~Hz},{ }^{5} \mathrm{~J}=0.8 \mathrm{~Hz}\right.$, $\left.1 \mathrm{H}, \mathrm{t}^{\prime}-\mathrm{H}\right), 7.74\left(\mathrm{ddd},{ }^{3} J=8.0 \mathrm{~Hz},{ }^{3} J=4.8 \mathrm{~Hz},{ }^{4} J=1.8 \mathrm{~Hz}, 1 \mathrm{H}, \mathrm{b}^{\prime}-\mathrm{H}\right), 7.94\left(\mathrm{~d},{ }^{3} J=2.0 \mathrm{~Hz}, 1 \mathrm{H}, \mathrm{j}^{\prime}-\right.$ H), $8.14\left(\mathrm{~d},{ }^{3} J=8.2 \mathrm{~Hz}, 1 \mathrm{H}, 8^{\prime}-\mathrm{H}\right), 8.25\left(\mathrm{t},{ }^{3} J=8.0 \mathrm{~Hz}, 1 \mathrm{H}, \mathrm{f}^{\prime}-\mathrm{H}\right), 8.27\left(\mathrm{td},{ }^{3} J=8.0 \mathrm{~Hz},{ }^{4} J=1.8\right.$ $\left.\mathrm{Hz}, 1 \mathrm{H}, \mathrm{c}^{\prime}-\mathrm{H}\right), 8.38\left(\mathrm{~d},{ }^{3} J=8.2 \mathrm{~Hz}, 1 \mathrm{H}, 6^{\prime}-\mathrm{H}\right), 8.40\left(\mathrm{dd},{ }^{3} \mathrm{~J}=8.0 \mathrm{~Hz},{ }^{4} \mathrm{~J}=2.0 \mathrm{~Hz}, 1 \mathrm{H}, \mathrm{i}^{\prime}-\mathrm{H}\right), 8.46$ $\left(\mathrm{dd},{ }^{3} J=8.2 \mathrm{~Hz},{ }^{4} J=1.2 \mathrm{~Hz}, 1 \mathrm{H}, 5^{\prime}-\mathrm{H}\right), 8.48\left(\mathrm{dd},{ }^{3} J=8.0 \mathrm{~Hz},{ }^{4} J=1.2 \mathrm{~Hz}, 1 \mathrm{H}, \mathrm{g}^{\prime}-\mathrm{H}\right), 8.58\left(\mathrm{dd},{ }^{3} J\right.$ $\left.=8.0 \mathrm{~Hz},{ }^{4} J=1.2 \mathrm{~Hz}, 1 \mathrm{H}, \mathrm{e}^{\prime}-\mathrm{H}\right), 8.63\left(\mathrm{~d},{ }^{3} J=8.0 \mathrm{~Hz}, 1 \mathrm{H}, \mathrm{h}^{\prime}-\mathrm{H}\right), 8.70\left(\mathrm{ddd},{ }^{3} J=8.0 \mathrm{~Hz},{ }^{4} J=1.8\right.$ $\left.\mathrm{Hz},{ }^{5} J=0.8 \mathrm{~Hz}, 1 \mathrm{H}, \mathrm{d}^{\prime}-\mathrm{H}\right), 9.00\left(\mathrm{~d},{ }^{3} J=8.2 \mathrm{~Hz}, 1 \mathrm{H}, 7^{\prime}-\mathrm{H}\right), 9.14\left(\mathrm{~s}, 1 \mathrm{H}, 4^{\prime}-\mathrm{H}\right) \mathrm{ppm}$. Elemental analysis: Anal. Calcd for $\mathrm{C}_{71} \mathrm{H}_{49} \mathrm{HgN}_{5} \cdot 2 \mathrm{ClO}_{4}$ : $\mathrm{C}, 62.17$; H, 3.60; N, 5.11, Found: $\mathrm{C}, 62.34 ; \mathrm{H}$, 3.68; N, 5.43. ESI-MS: $m / z(\%) 586.9(100)[\mathrm{Hg}(\mathbf{4})]^{2+}$. 


\section{NMR Spectra: ${ }^{1} \mathrm{H},{ }^{13} \mathrm{C},{ }^{1} \mathrm{H}-{ }^{1} \mathrm{H}$ COSY}

For the numbering of protons, check the individual experimental procedure.
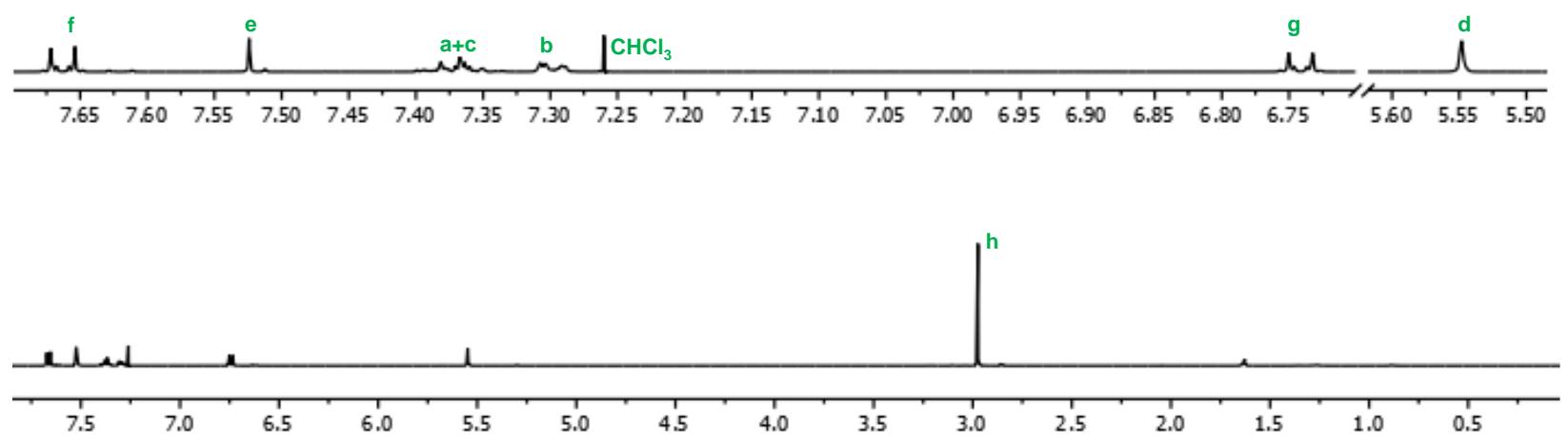

Figure S1: ${ }^{1} \mathrm{H}$ NMR spectrum $\left(\mathrm{CDCl}_{3}, 500 \mathrm{MHz}, 298 \mathrm{~K}\right)$ of product 7.
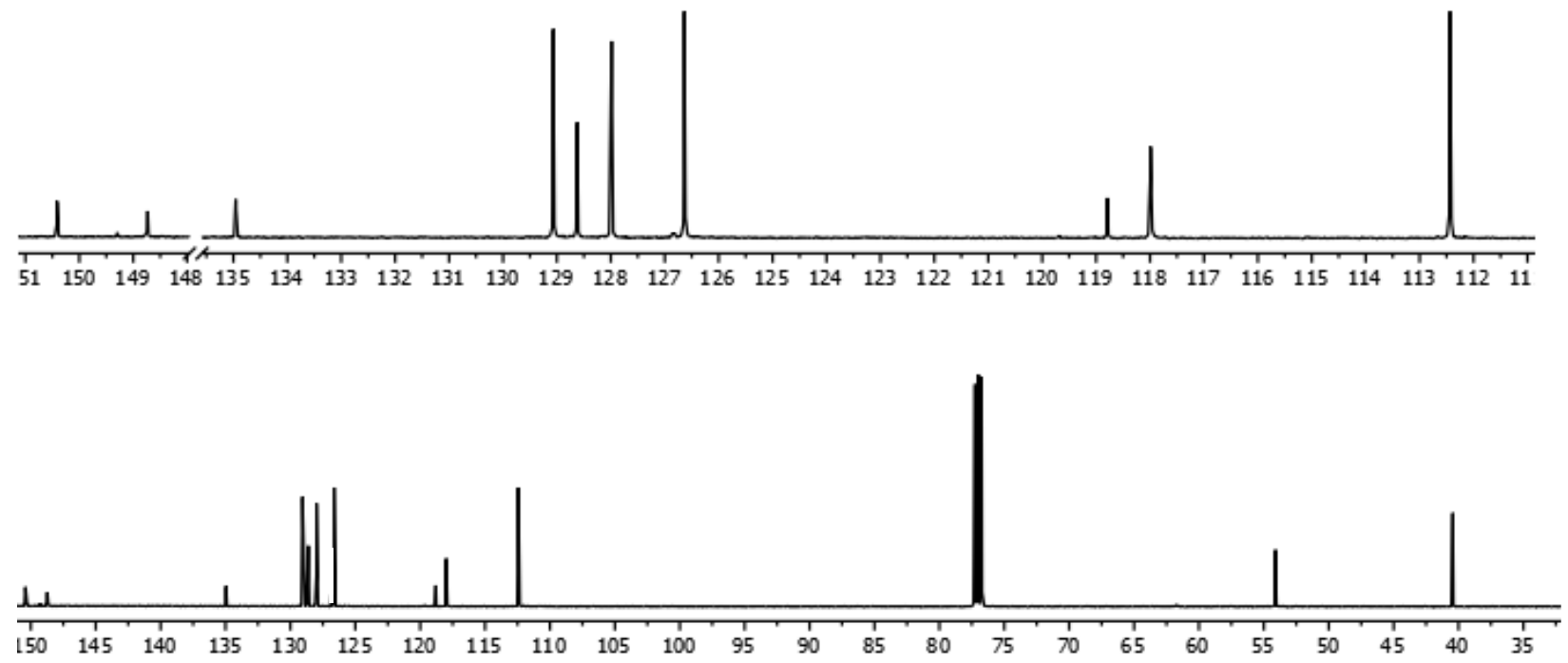

Figure S2: ${ }^{13} \mathrm{C}$ NMR spectrum $\left(\mathrm{CDCl}_{3}, 125 \mathrm{MHz}, 298 \mathrm{~K}\right)$ of product 7.

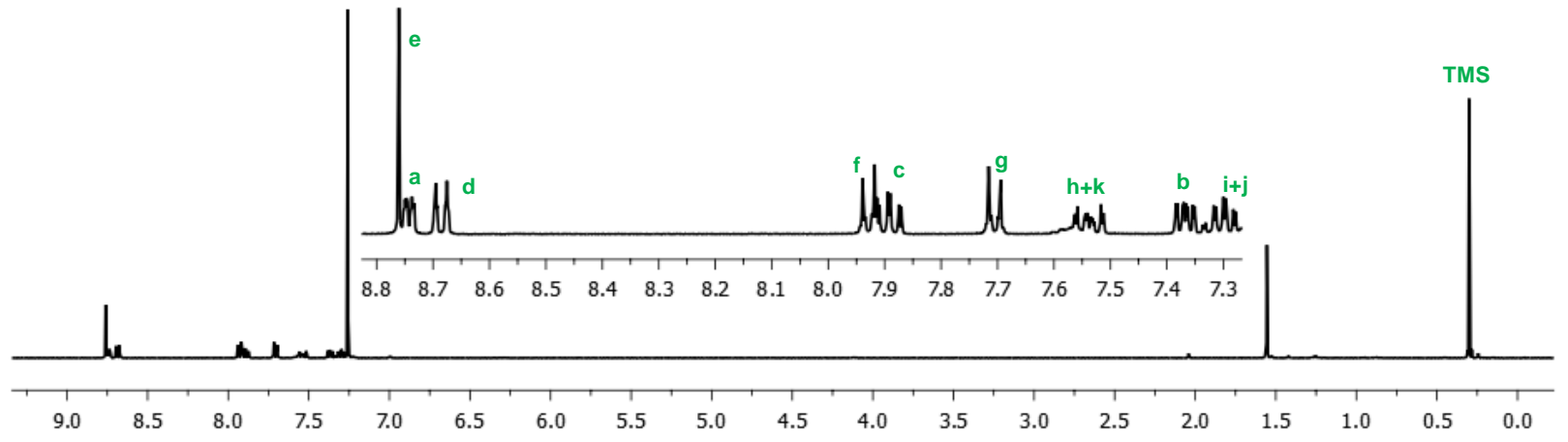

Figure S3: ${ }^{1} \mathrm{H} \mathrm{NMR}$ spectrum $\left(\mathrm{CDCl}_{3}, 400 \mathrm{MHz}, 298 \mathrm{~K}\right)$ of ligand 9. 

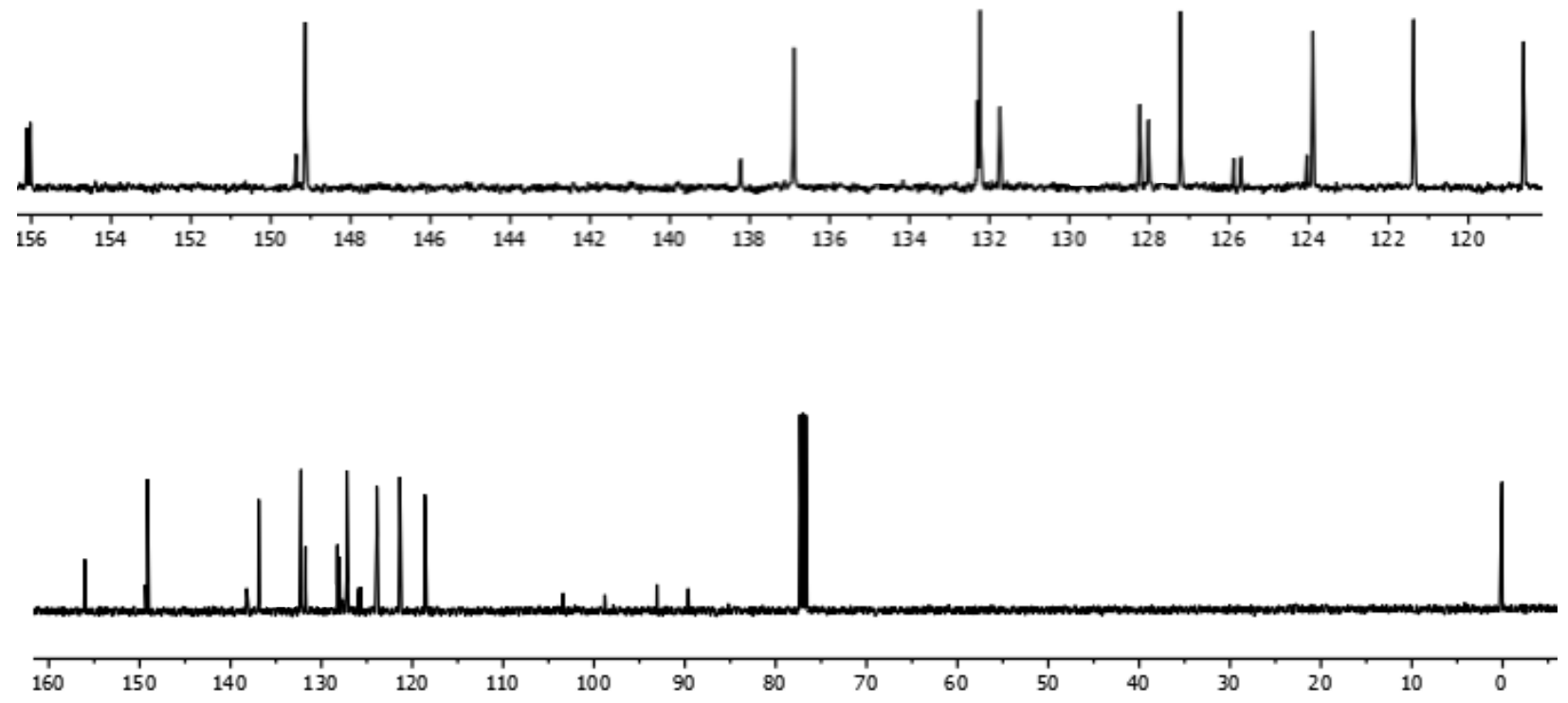

Figure S4: ${ }^{13} \mathrm{C}$ NMR spectrum $\left(\mathrm{CDCl}_{3}, 100 \mathrm{MHz}, 298 \mathrm{~K}\right)$ of ligand 9.
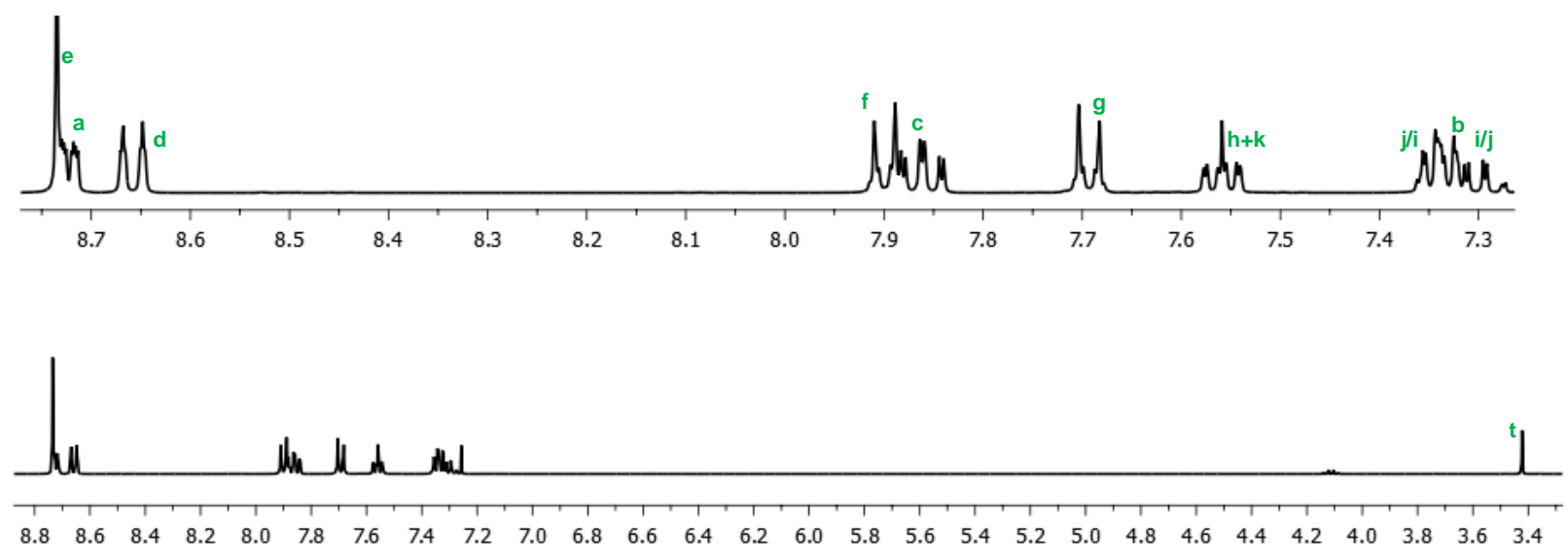

Figure S5: ${ }^{1} \mathrm{H}$ NMR spectrum $\left(\mathrm{CDCl}_{3}, 400 \mathrm{MHz}, 298 \mathrm{~K}\right)$ of ligand 10.

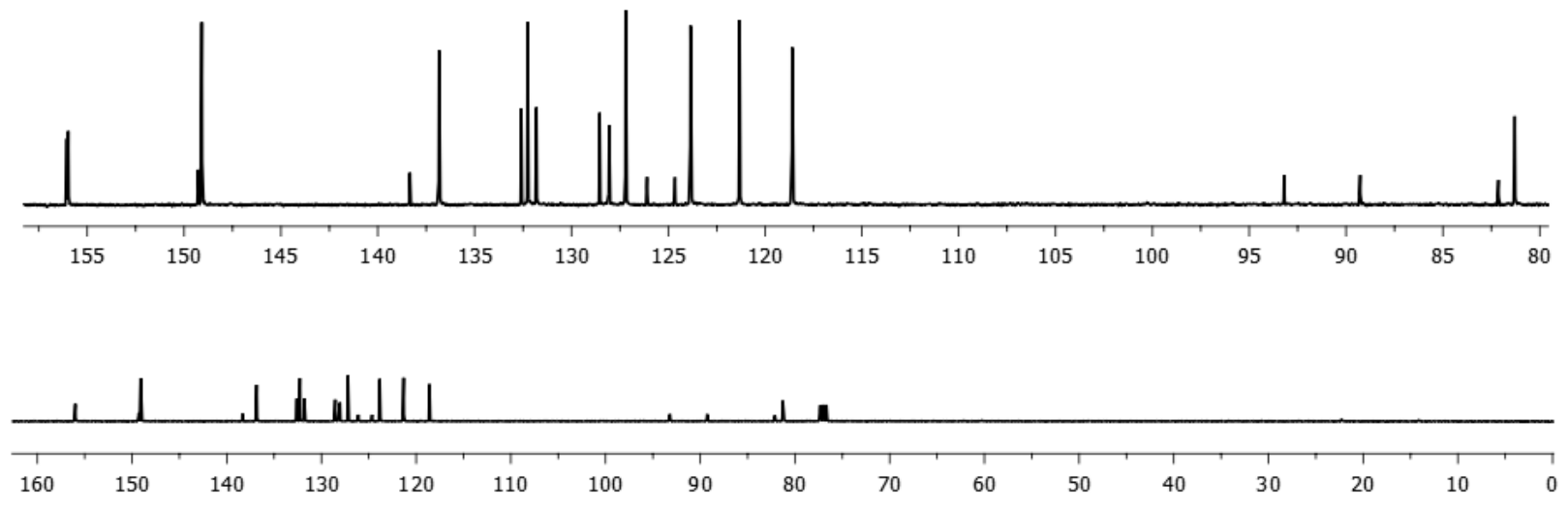

Figure S6: ${ }^{13} \mathrm{C}$ NMR spectrum $\left(\mathrm{CDCl}_{3}, 100 \mathrm{MHz}, 298 \mathrm{~K}\right)$ of ligand 10. 


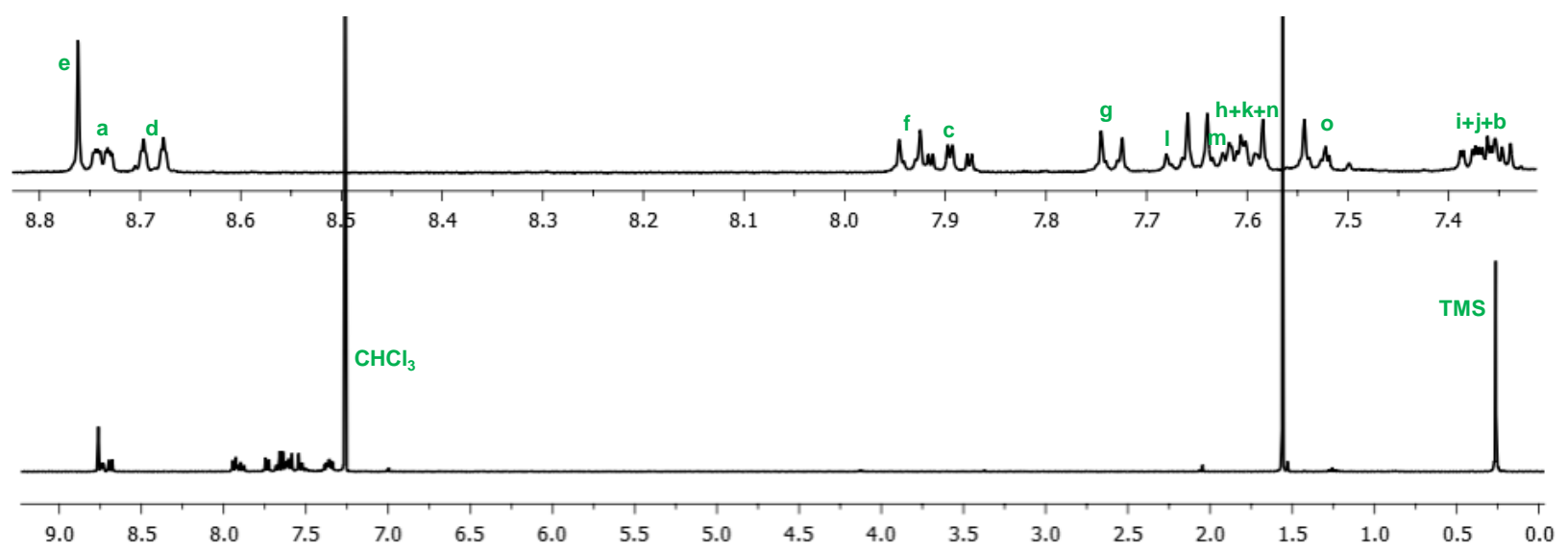

Figure S7: ${ }^{1} \mathrm{H}$ NMR spectrum $\left(\mathrm{CDCl}_{3}, 400 \mathrm{MHz}, 298 \mathrm{~K}\right)$ of ligand 12.
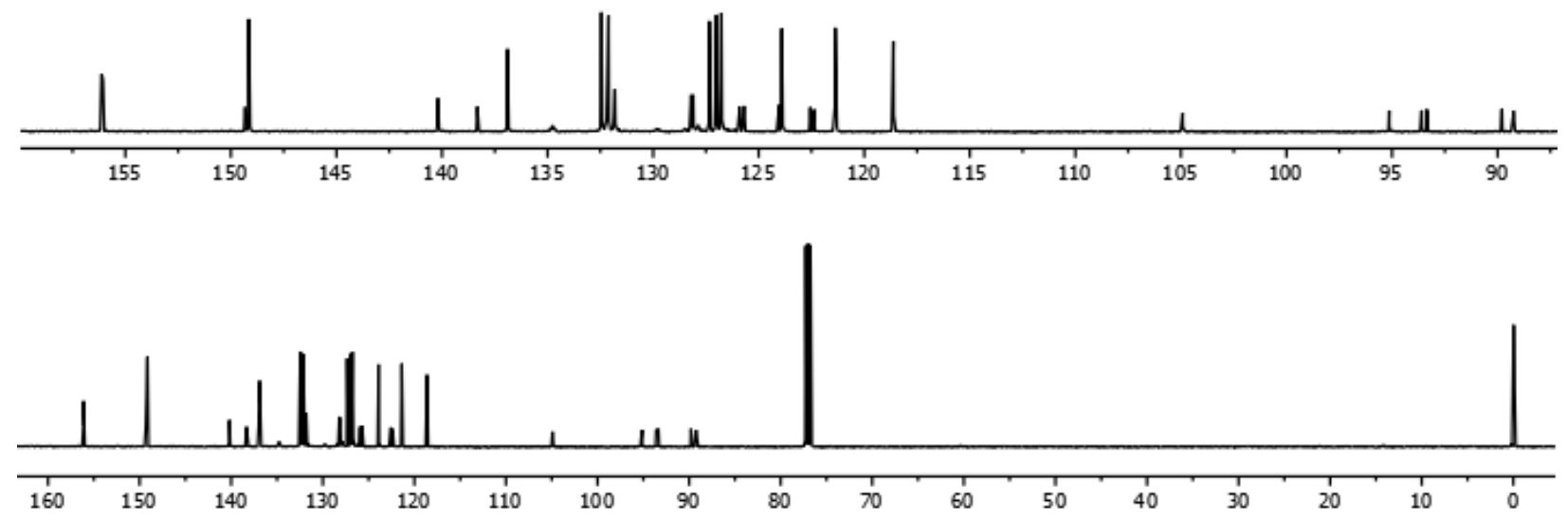

Figure S8: ${ }^{13} \mathrm{C}$ NMR spectrum $\left(\mathrm{CDCl}_{3}, 100 \mathrm{MHz}, 298 \mathrm{~K}\right)$ of ligand 12.
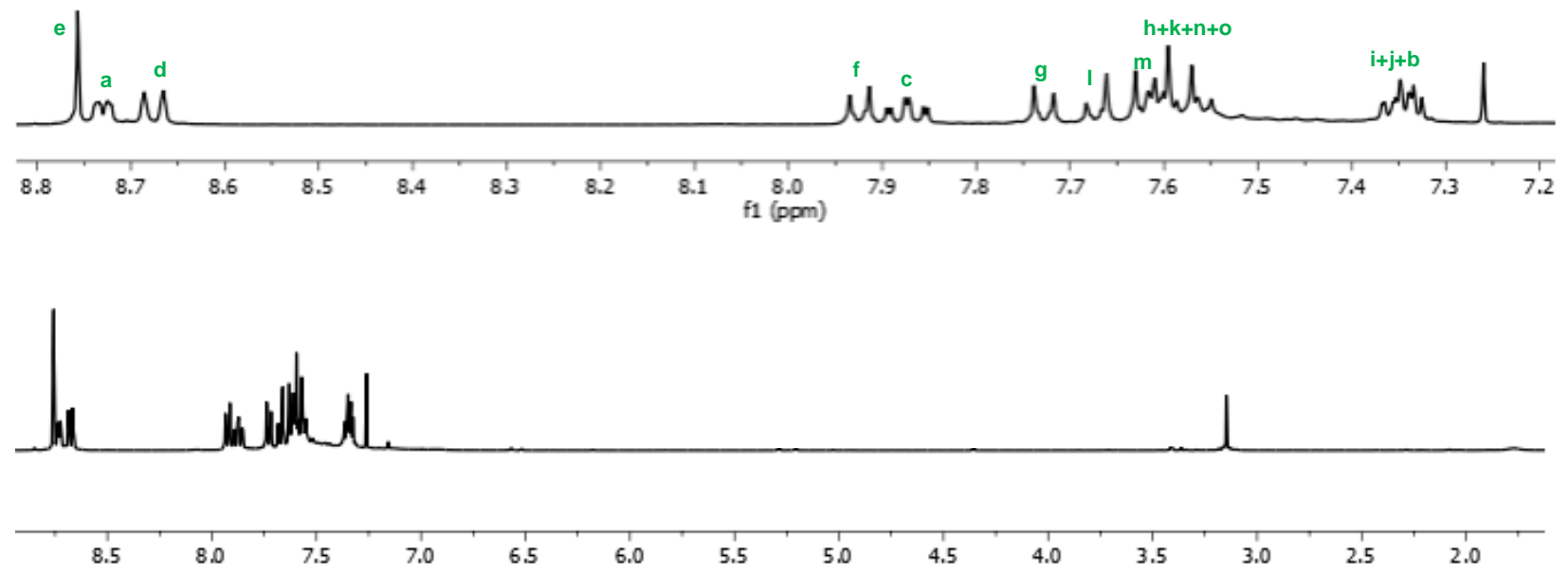

Figure S9: ${ }^{1} \mathrm{H}$ NMR spectrum $\left(\mathrm{CDCl}_{3}, 400 \mathrm{MHz}, 298 \mathrm{~K}\right)$ of ligand 13. 

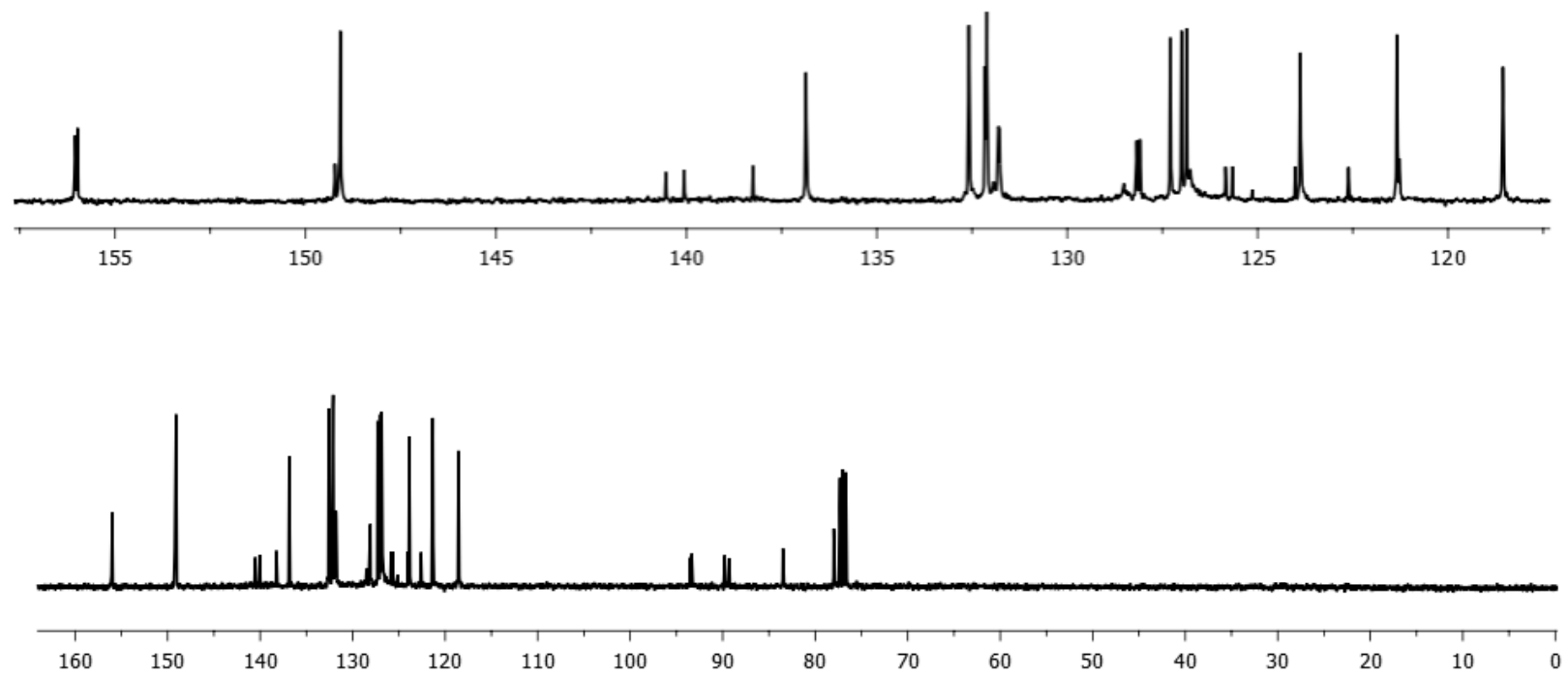

Figure S10: ${ }^{13} \mathrm{C}$ NMR spectrum $\left(\mathrm{CDCl}_{3}, 100 \mathrm{MHz}, 298 \mathrm{~K}\right)$ of ligand 13.

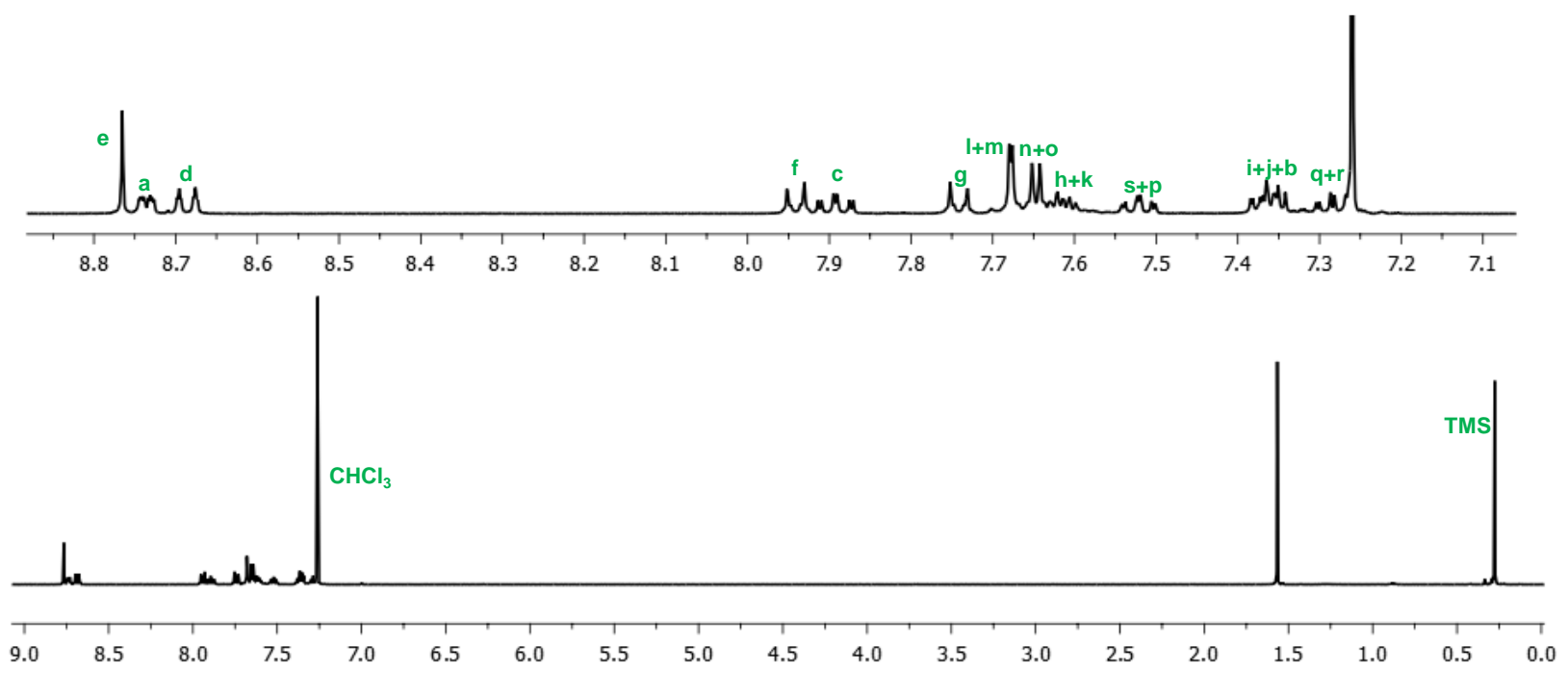

Figure S11: ${ }^{1} \mathrm{H}$ NMR spectrum $\left(\mathrm{CDCl}_{3}, 400 \mathrm{MHz}, 298 \mathrm{~K}\right)$ of ligand 14. 

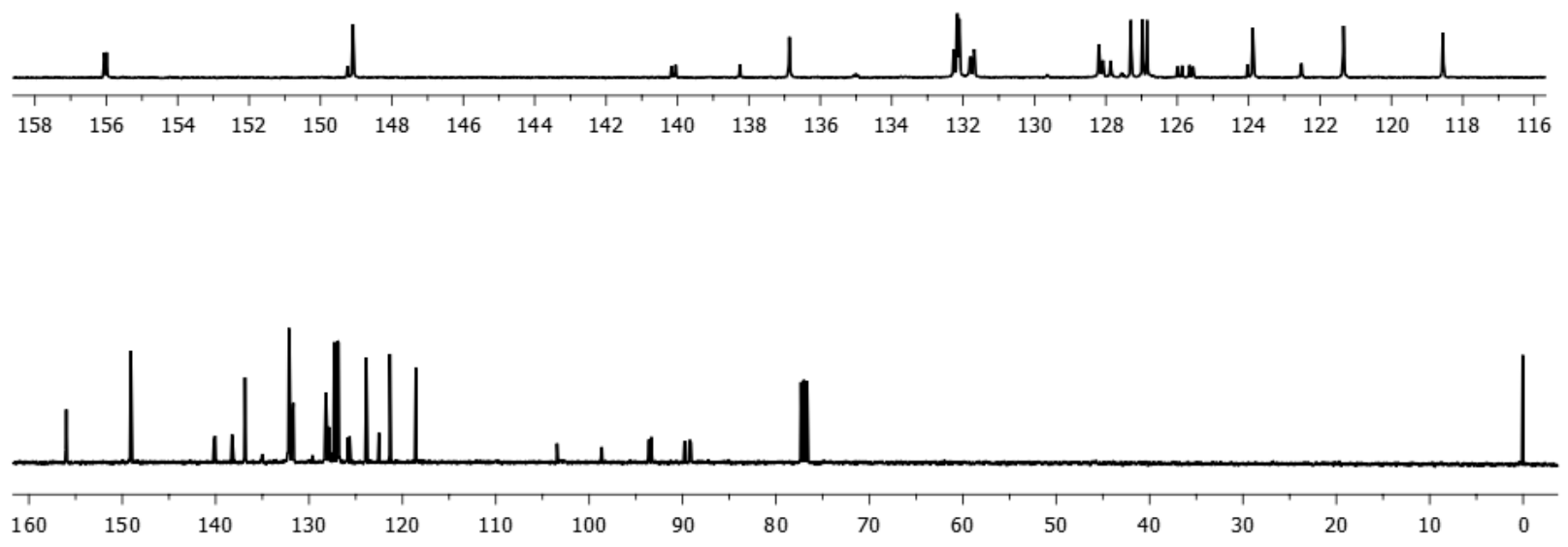

Figure S12: ${ }^{13} \mathrm{C}$ NMR spectrum $\left(\mathrm{CDCl}_{3}, 100 \mathrm{MHz}, 298 \mathrm{~K}\right)$ of ligand 14.

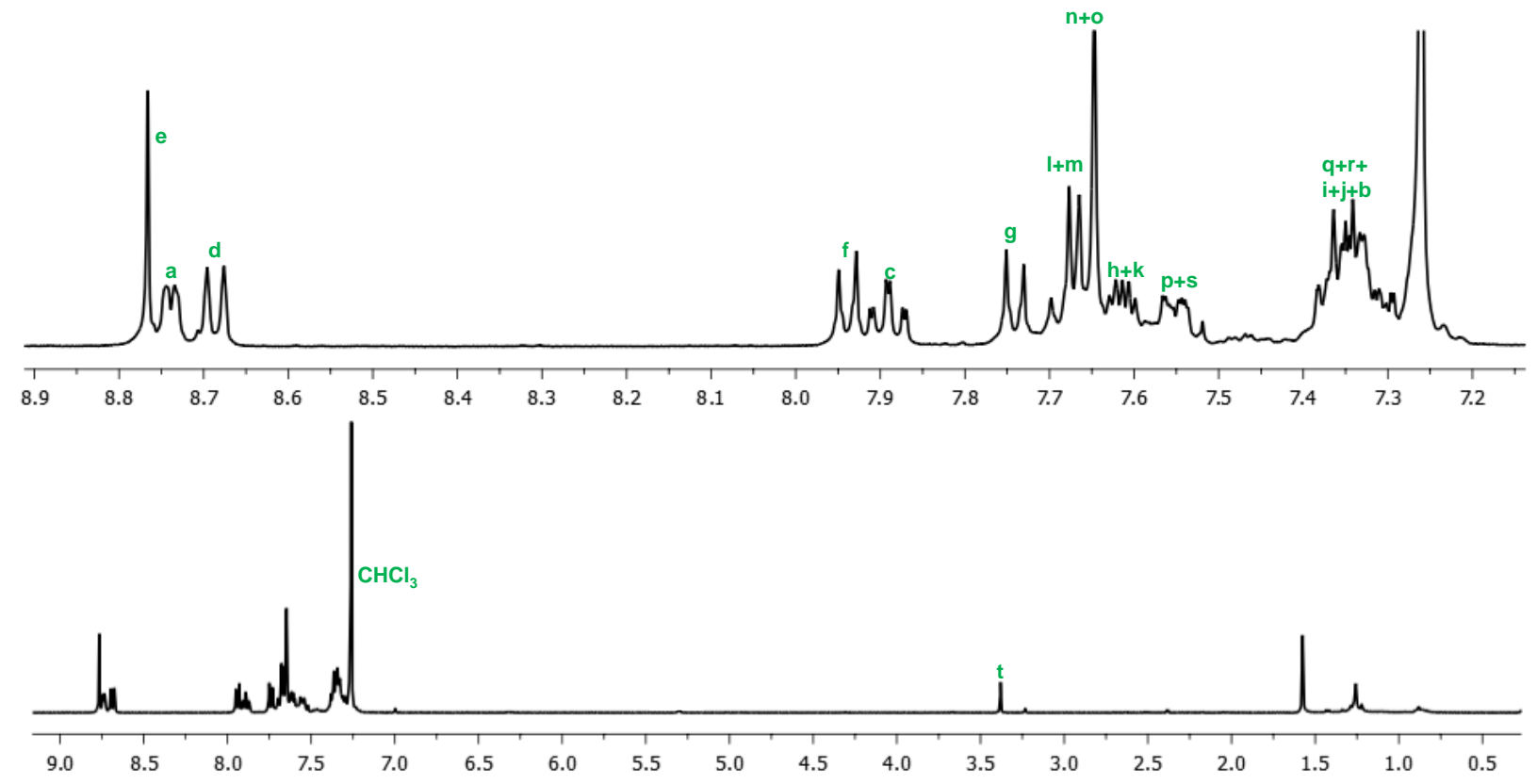

Figure S13: ${ }^{1} \mathrm{H}$ NMR spectrum $\left(\mathrm{CDCl}_{3}, 400 \mathrm{MHz}, 298 \mathrm{~K}\right)$ of ligand 15. 

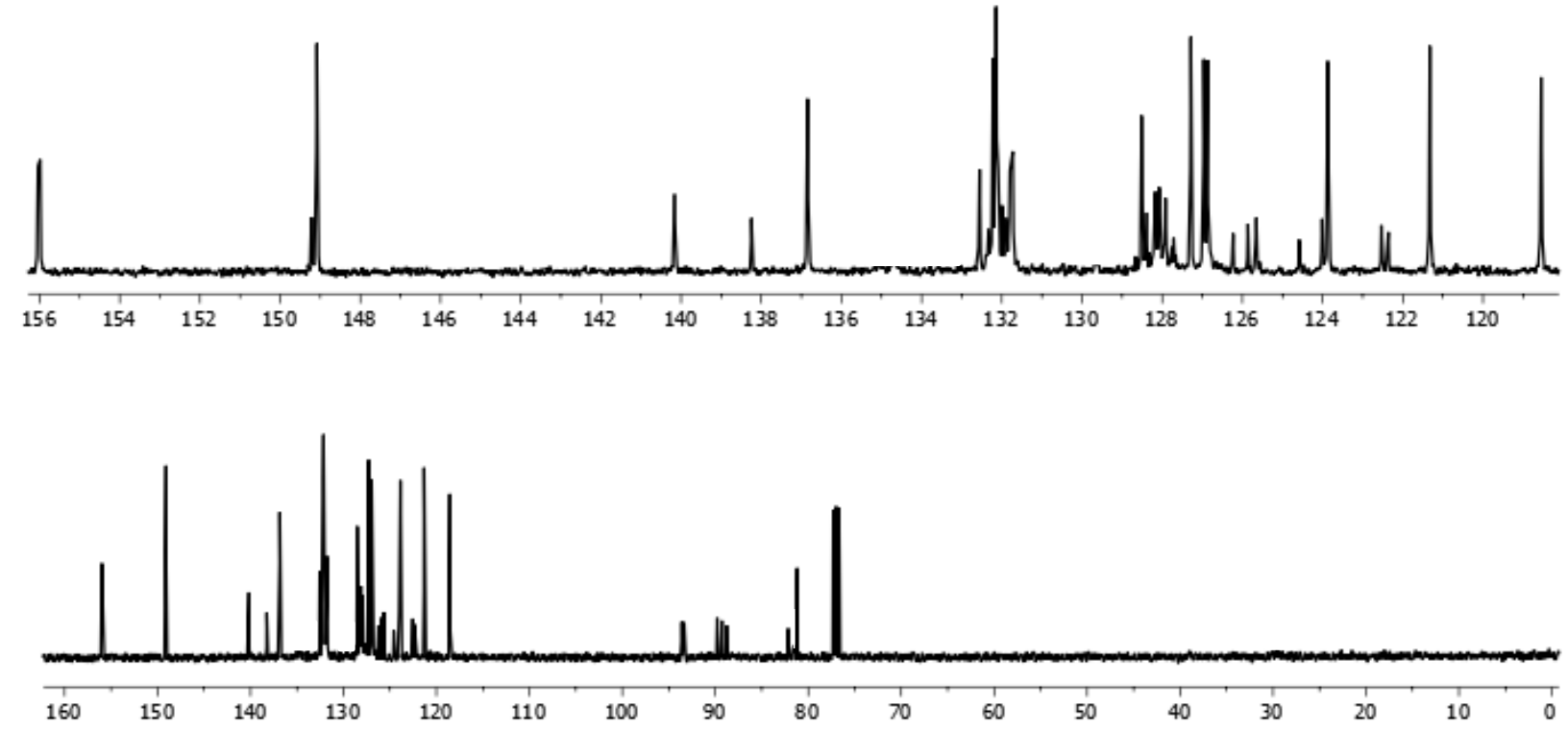

Figure S14: ${ }^{13} \mathrm{C}$ NMR spectrum $\left(\mathrm{CDCl}_{3}, 100 \mathrm{MHz}, 298 \mathrm{~K}\right)$ of ligand 15.

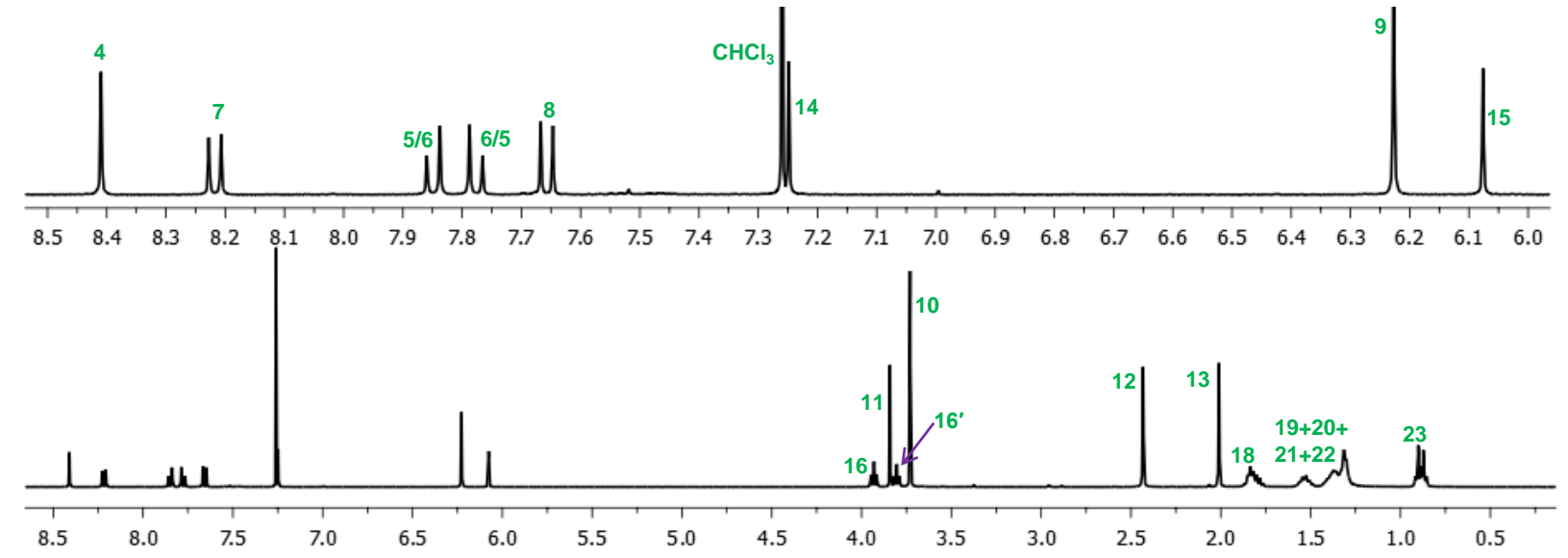

Figure S15: ${ }^{1} \mathrm{H}$ NMR spectrum $\left(\mathrm{CDCl}_{3}, 400 \mathrm{MHz}, 298 \mathrm{~K}\right)$ of ligand 16. 

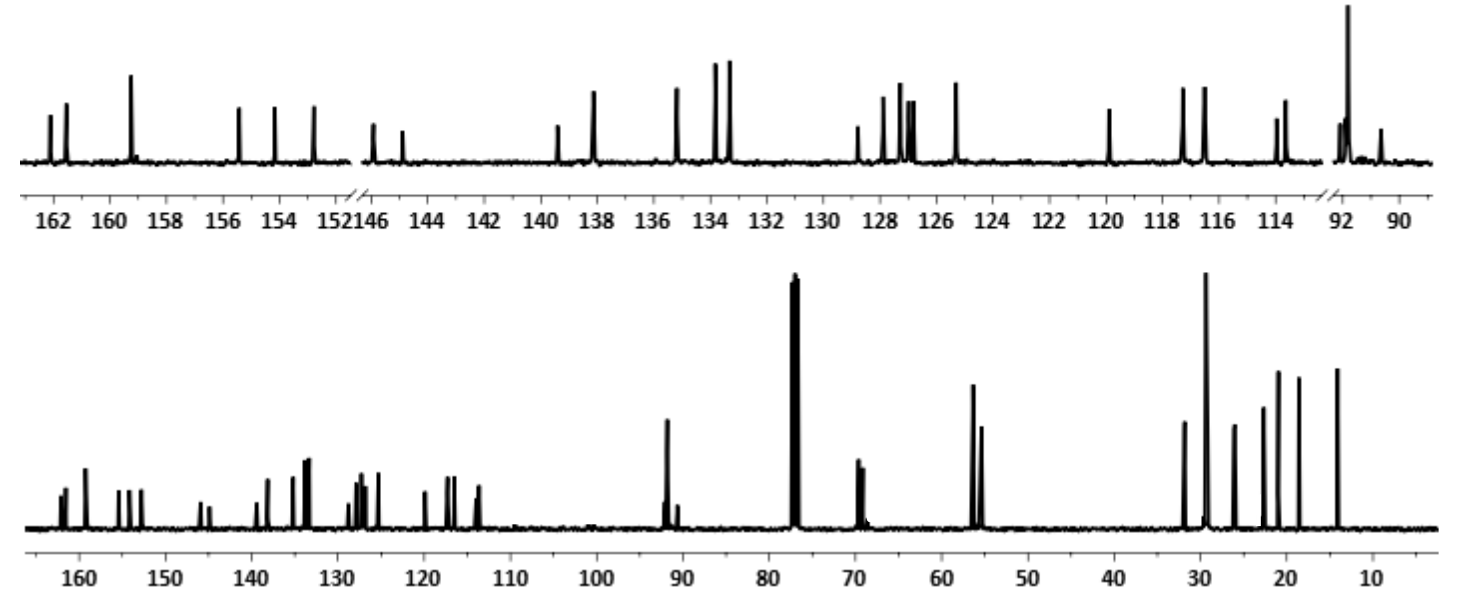

Figure S16: ${ }^{13} \mathrm{C}$ NMR spectrum $\left(\mathrm{CDCl}_{3}, 100 \mathrm{MHz}, 298 \mathrm{~K}\right)$ of ligand 16.

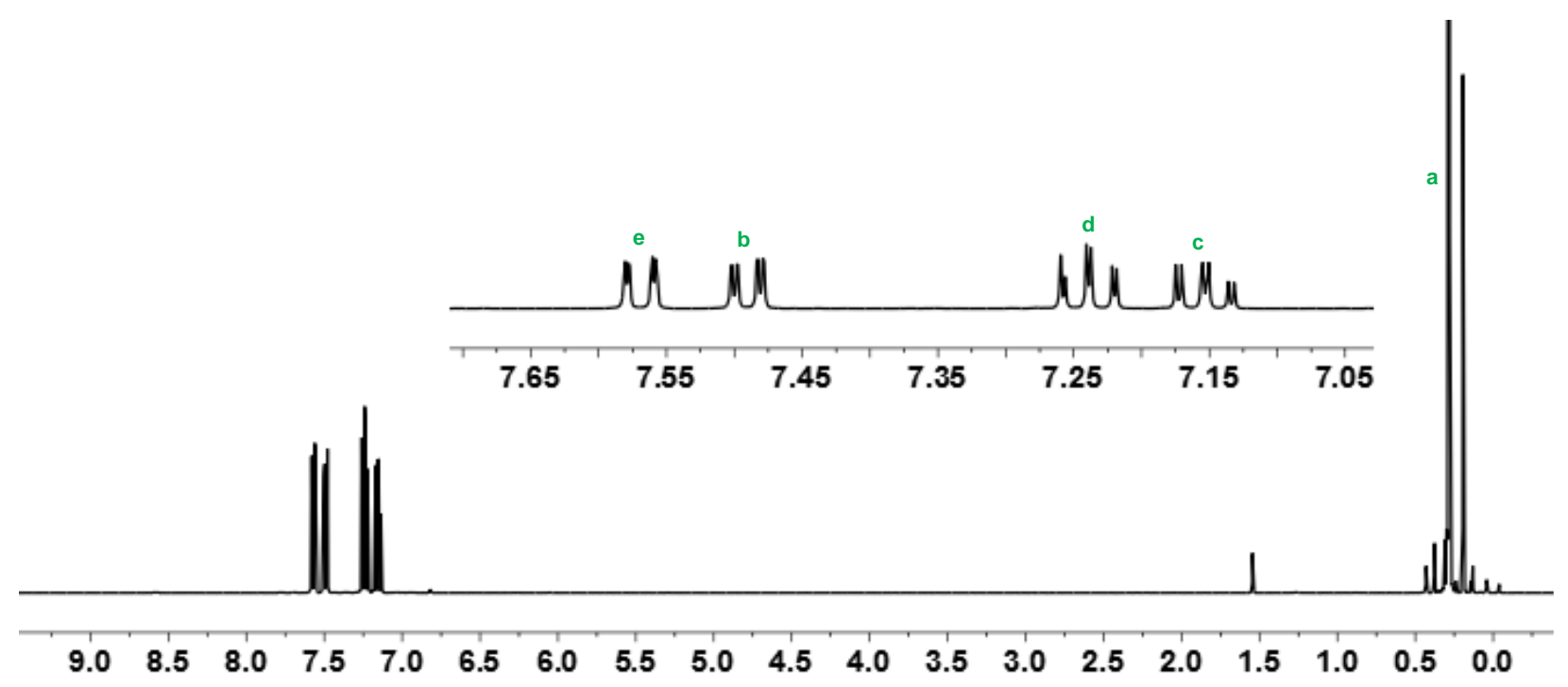

Figure S17: ${ }^{1} \mathrm{H}$ NMR spectrum $\left(\mathrm{CDCl}_{3}, 400 \mathrm{MHz}, 298 \mathrm{~K}\right)$ of ligand 17.

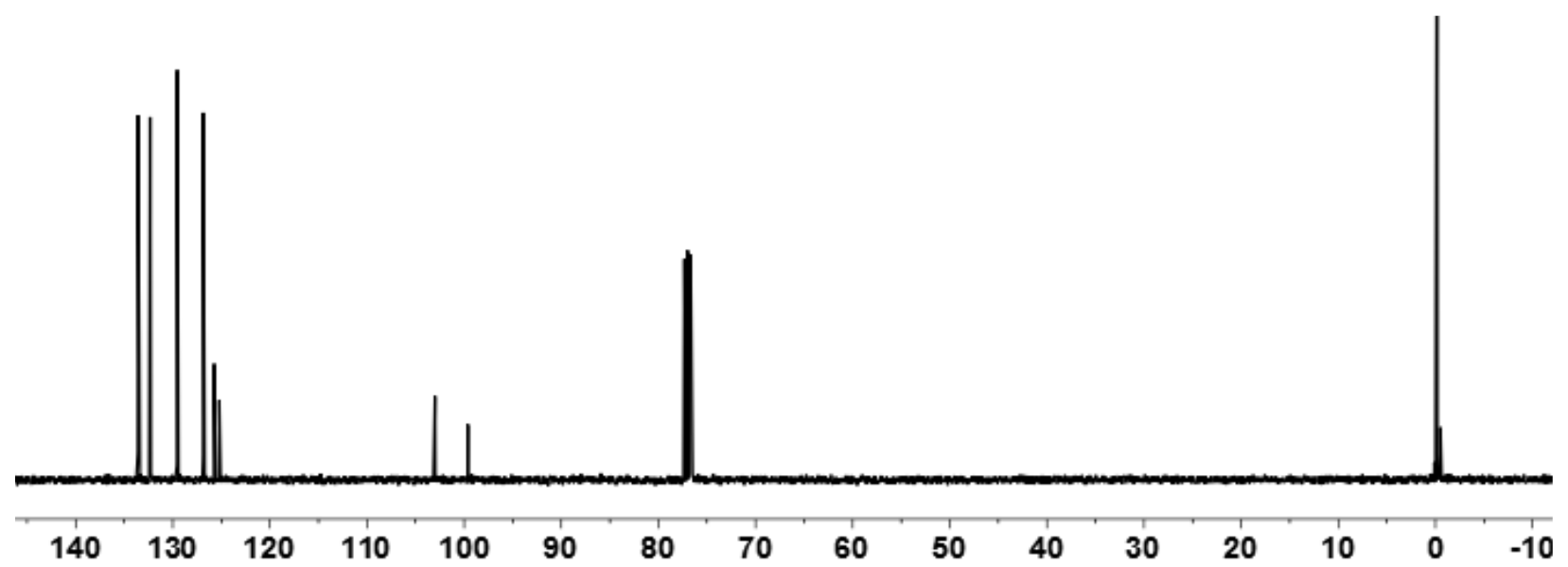

Figure S18: ${ }^{13} \mathrm{C}$ NMR spectrum $\left(\mathrm{CDCl}_{3}, 100 \mathrm{MHz}, 298 \mathrm{~K}\right)$ of ligand 17. 

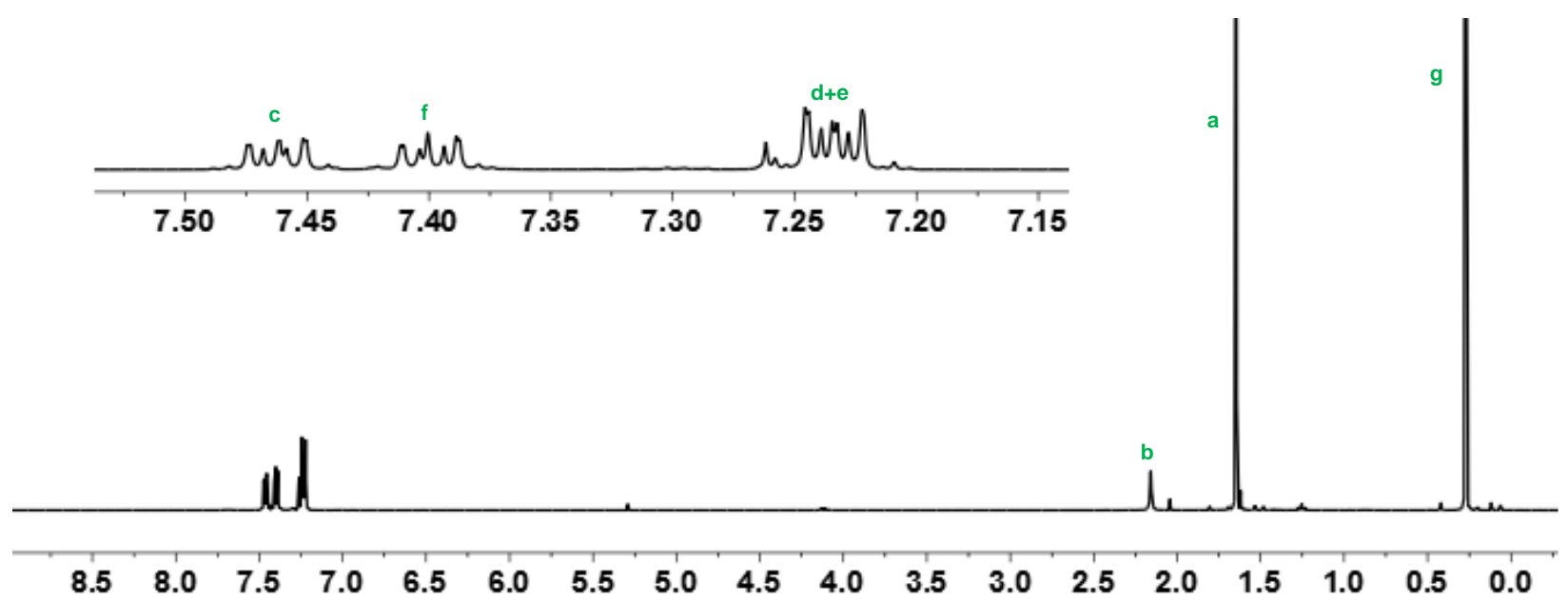

Figure S19: ${ }^{1} \mathrm{H}$ NMR spectrum $\left(\mathrm{CDCl}_{3}, 400 \mathrm{MHz}, 298 \mathrm{~K}\right)$ of ligand 18.

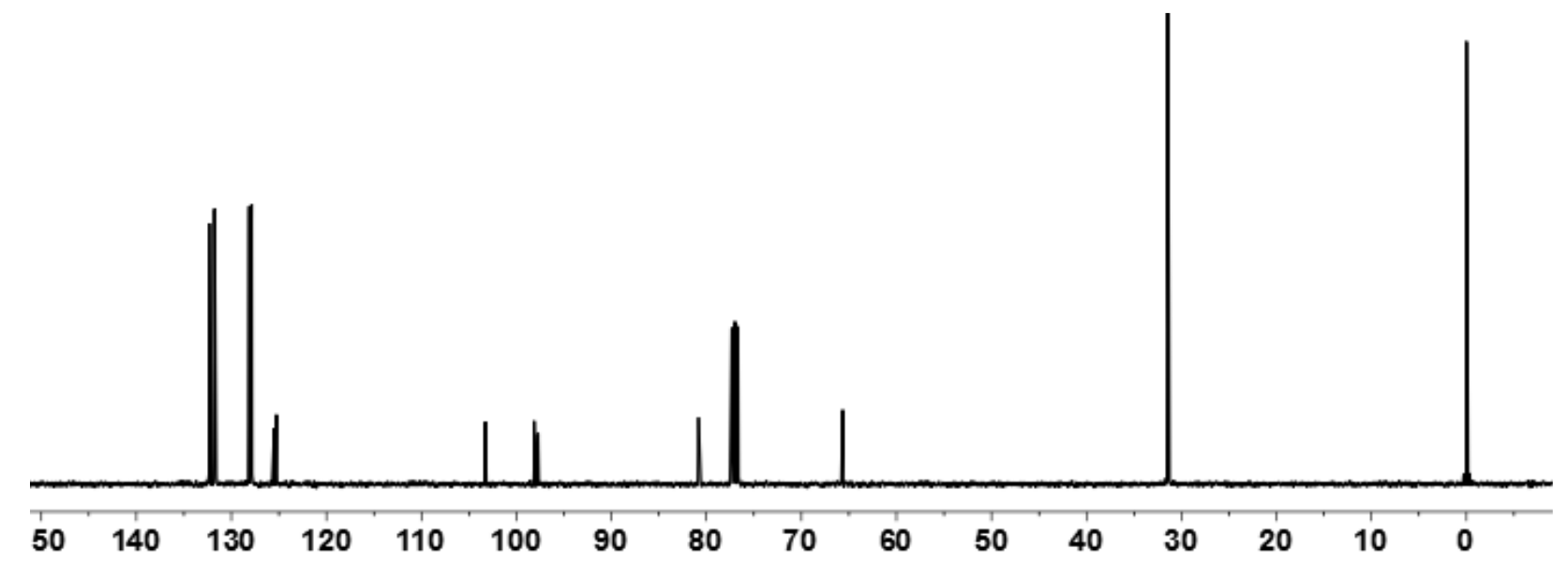

Figure S20: ${ }^{13} \mathrm{C}$ NMR spectrum $\left(\mathrm{CDCl}_{3}, 100 \mathrm{MHz}, 298 \mathrm{~K}\right)$ of ligand 18.
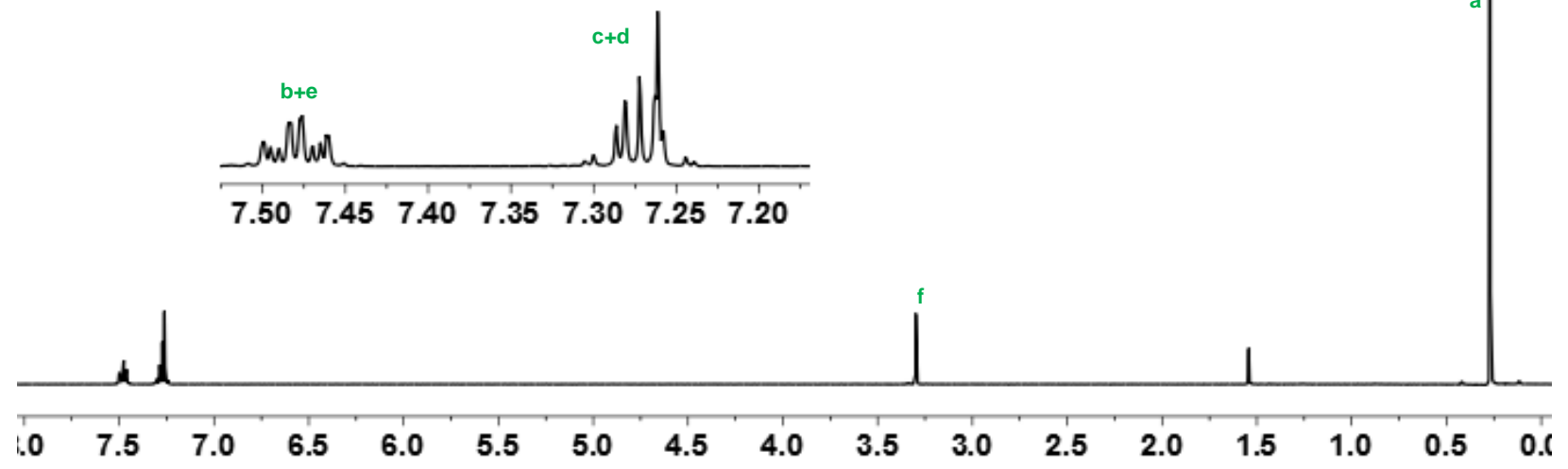

Figure S21: ${ }^{1} \mathrm{H}$ NMR spectrum $\left(\mathrm{CDCl}_{3}, 400 \mathrm{MHz}, 298 \mathrm{~K}\right)$ of ligand 19. 

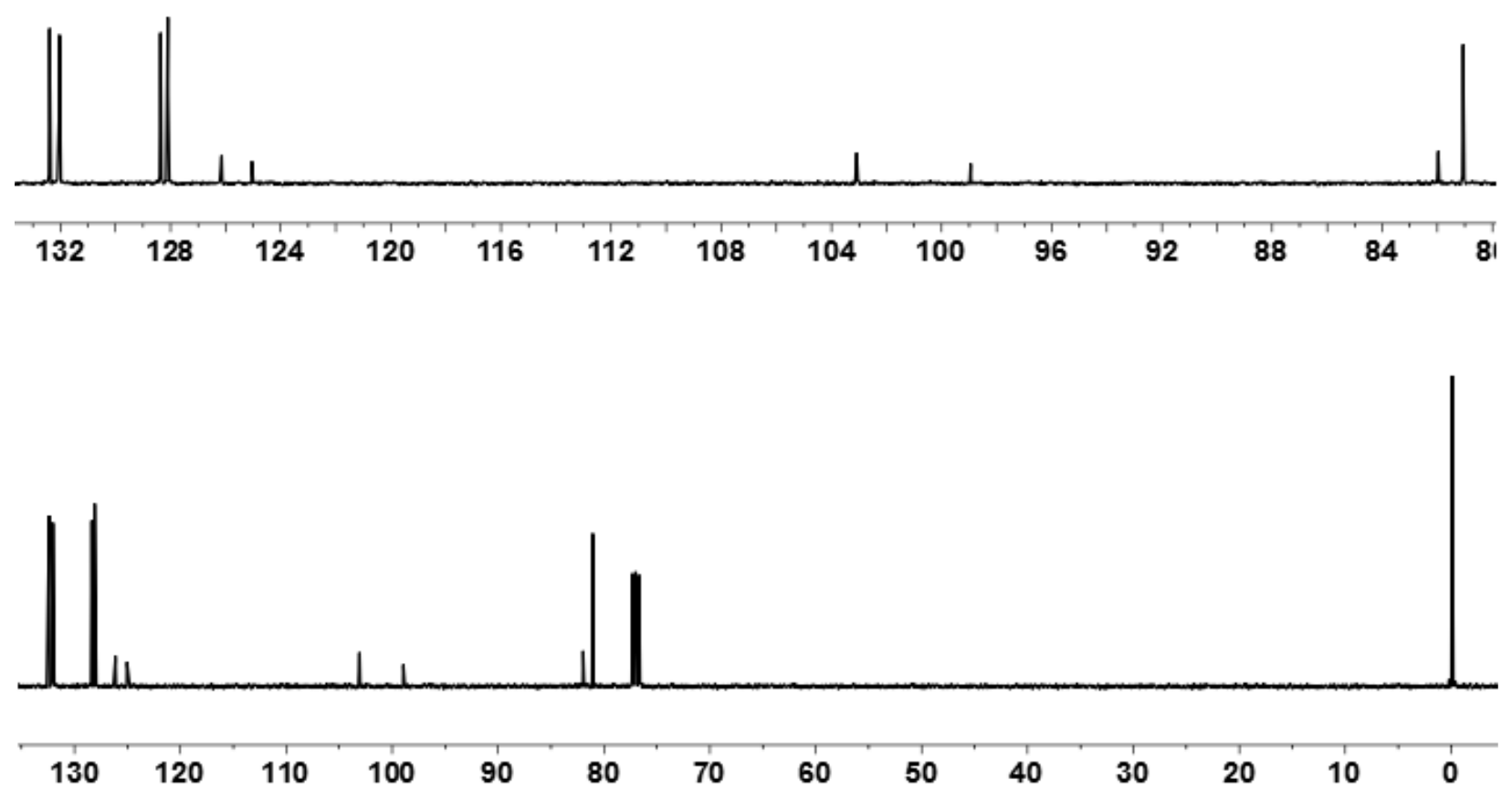

Figure S22: ${ }^{13} \mathrm{C}$ NMR spectrum $\left(\mathrm{CDCl}_{3}, 100 \mathrm{MHz}, 298 \mathrm{~K}\right)$ of ligand 19.

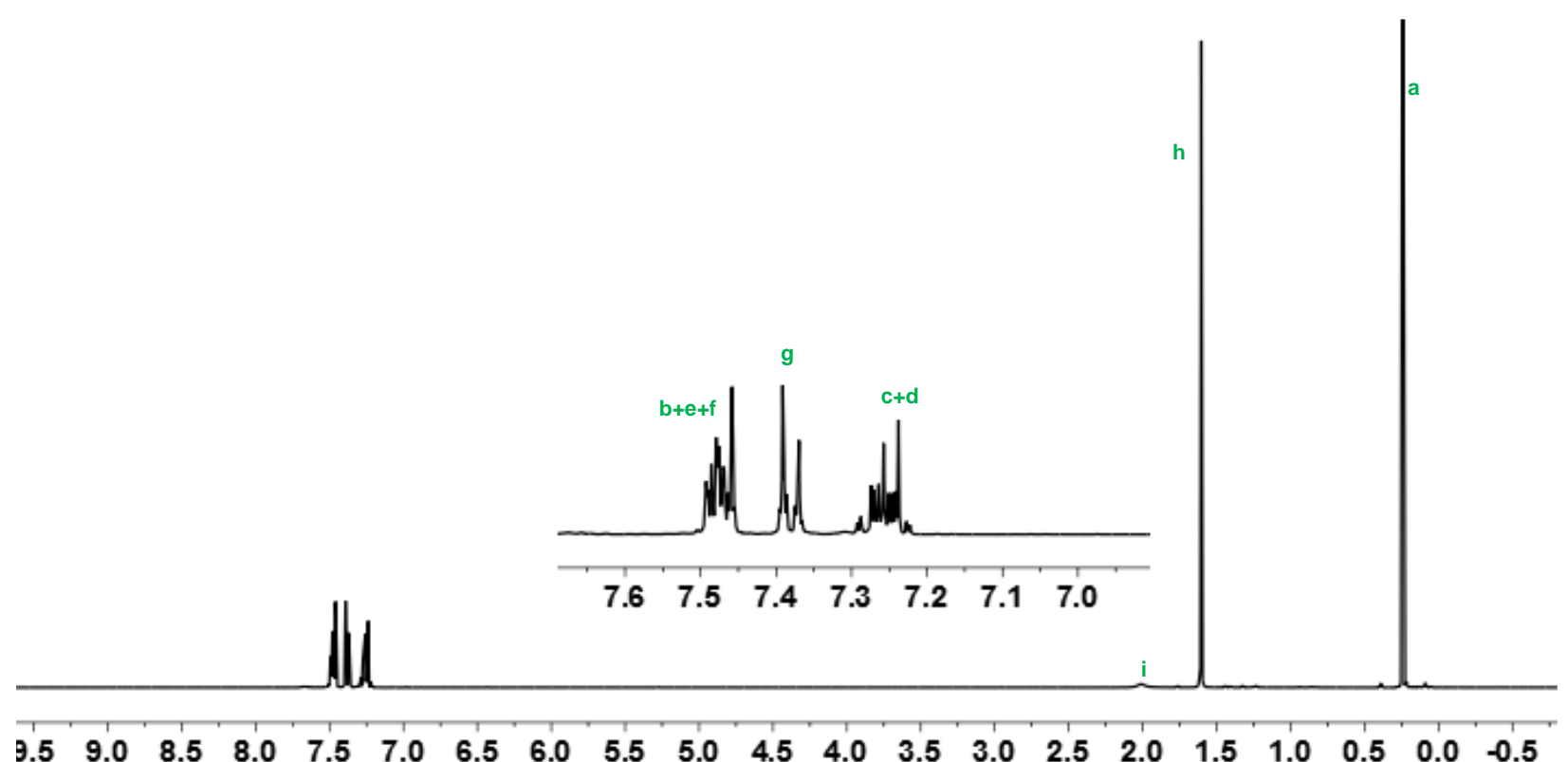

Figure S23: ${ }^{1} \mathrm{H}$ NMR spectrum $\left(\mathrm{CDCl}_{3}, 400 \mathrm{MHz}, 298 \mathrm{~K}\right)$ of ligand 20. 

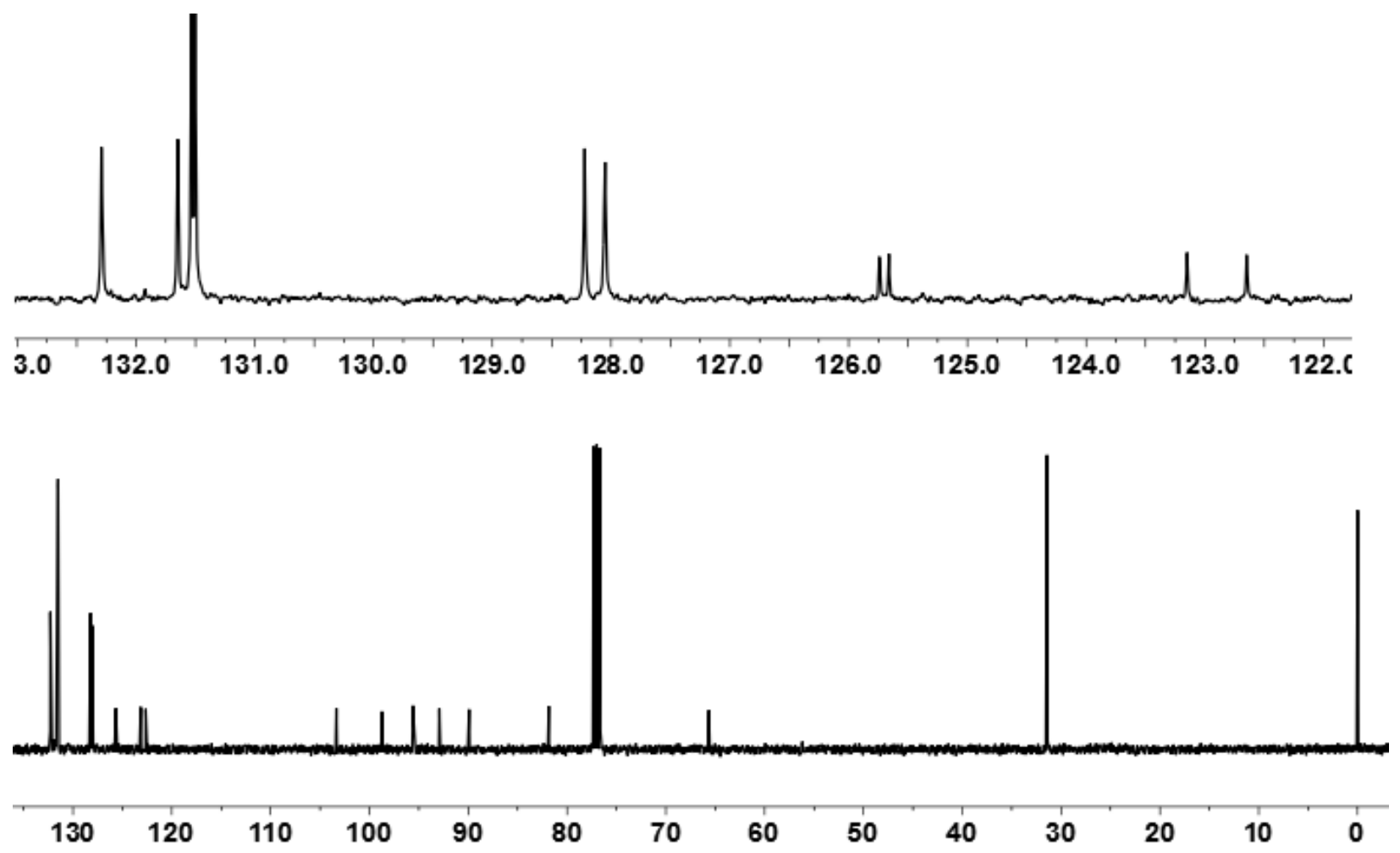

Figure S24: ${ }^{13} \mathrm{C}$ NMR spectrum $\left(\mathrm{CDCl}_{3}, 100 \mathrm{MHz}, 298 \mathrm{~K}\right)$ of ligand 20.

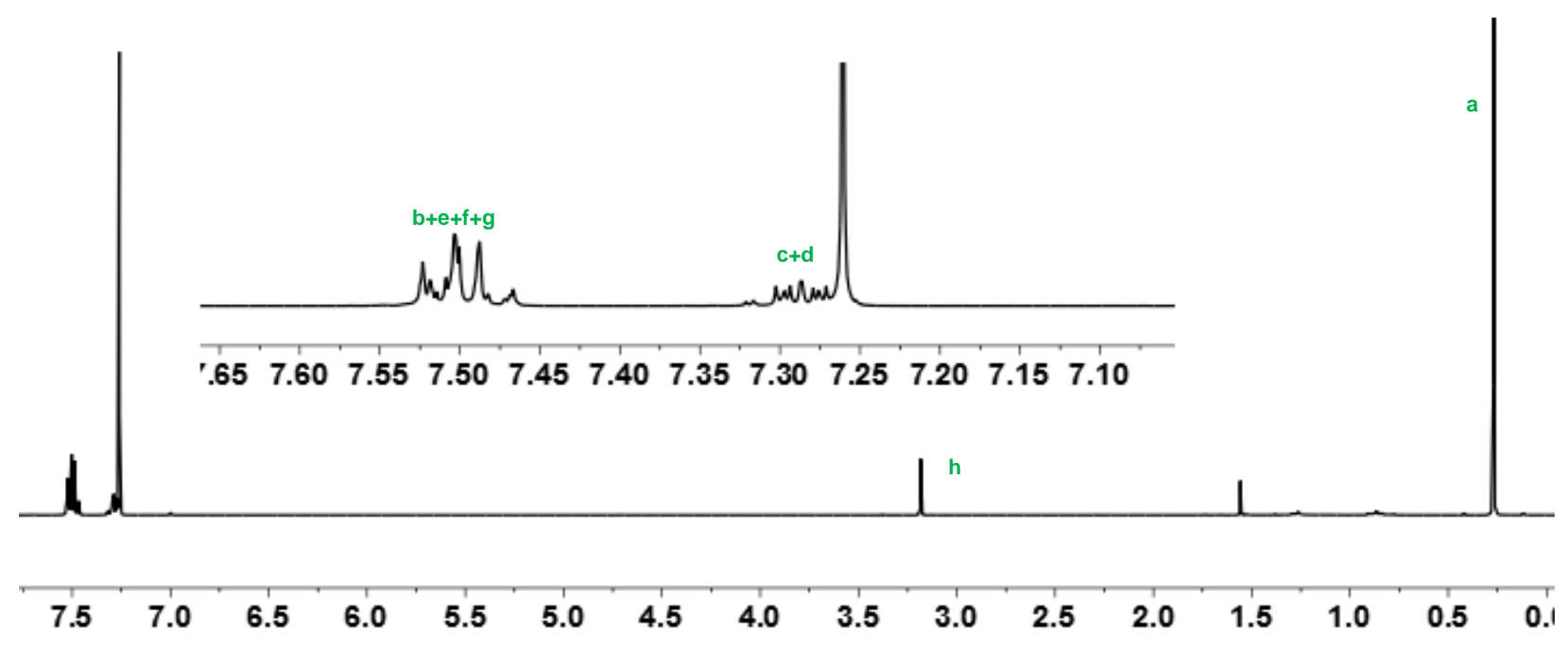

Figure S25: ${ }^{1} \mathrm{H}$ NMR spectrum $\left(\mathrm{CDCl}_{3}, 400 \mathrm{MHz}, 298 \mathrm{~K}\right)$ of ligand 21. 

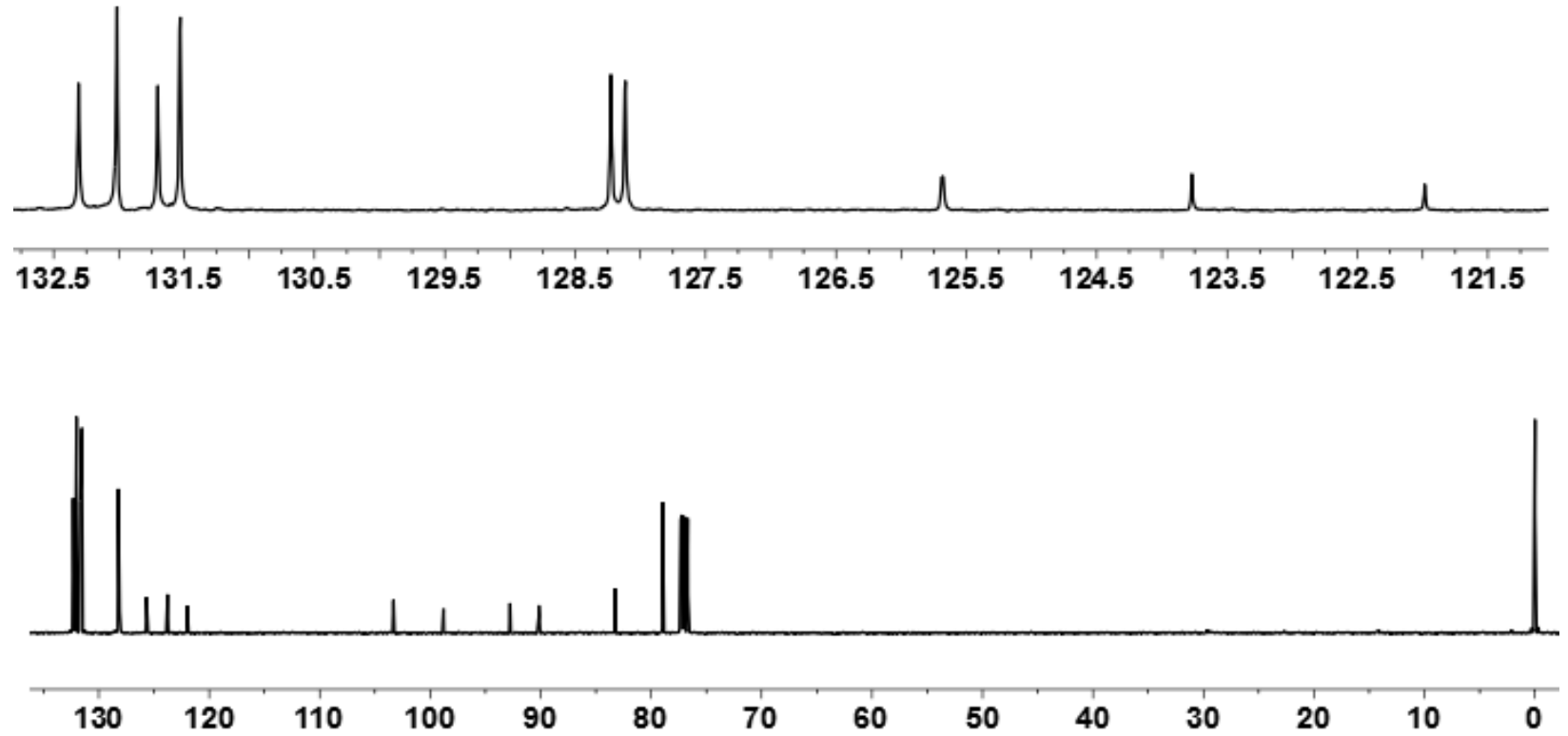

Figure S26: ${ }^{13} \mathrm{C}$ NMR spectrum $\left(\mathrm{CDCl}_{3}, 100 \mathrm{MHz}, 298 \mathrm{~K}\right)$ of ligand 21.

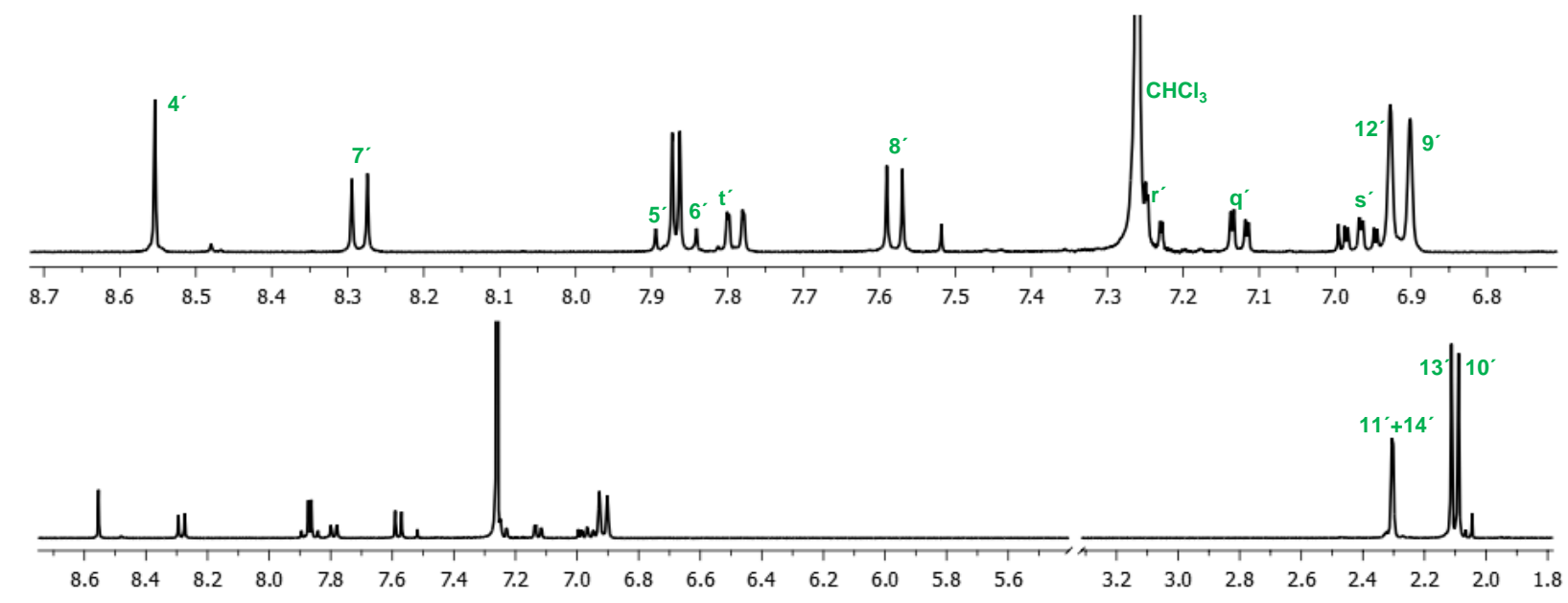

Figure S27: ${ }^{1} \mathrm{H}$ NMR spectrum $\left(\mathrm{CDCl}_{3}, 400 \mathrm{MHz}, 298 \mathrm{~K}\right)$ of ligand 22. 

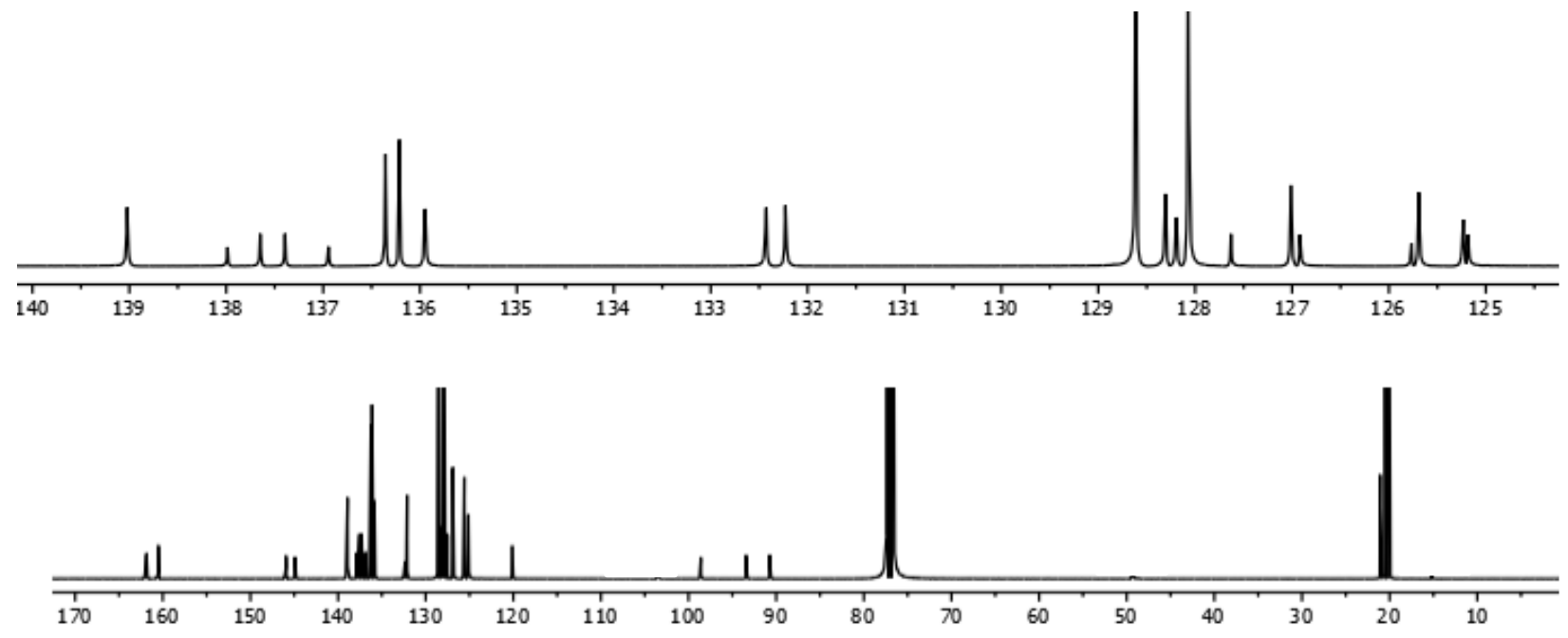

Figure S28: ${ }^{13} \mathrm{C}$ NMR spectrum $\left(\mathrm{CDCl}_{3}, 100 \mathrm{MHz}, 298 \mathrm{~K}\right)$ of ligand 22.
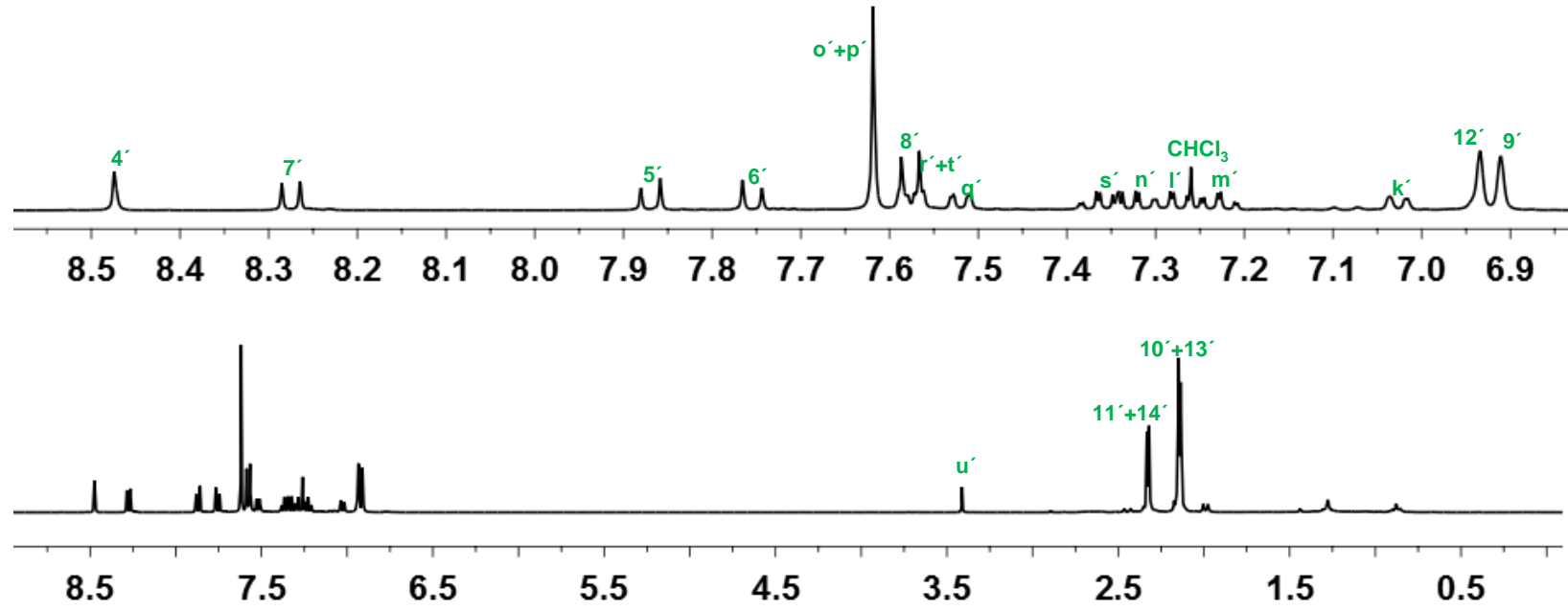

Figure S29: ${ }^{1} \mathrm{H}$ NMR spectrum $\left(\mathrm{CDCl}_{3}, 400 \mathrm{MHz}, 298 \mathrm{~K}\right)$ of ligand 23. 

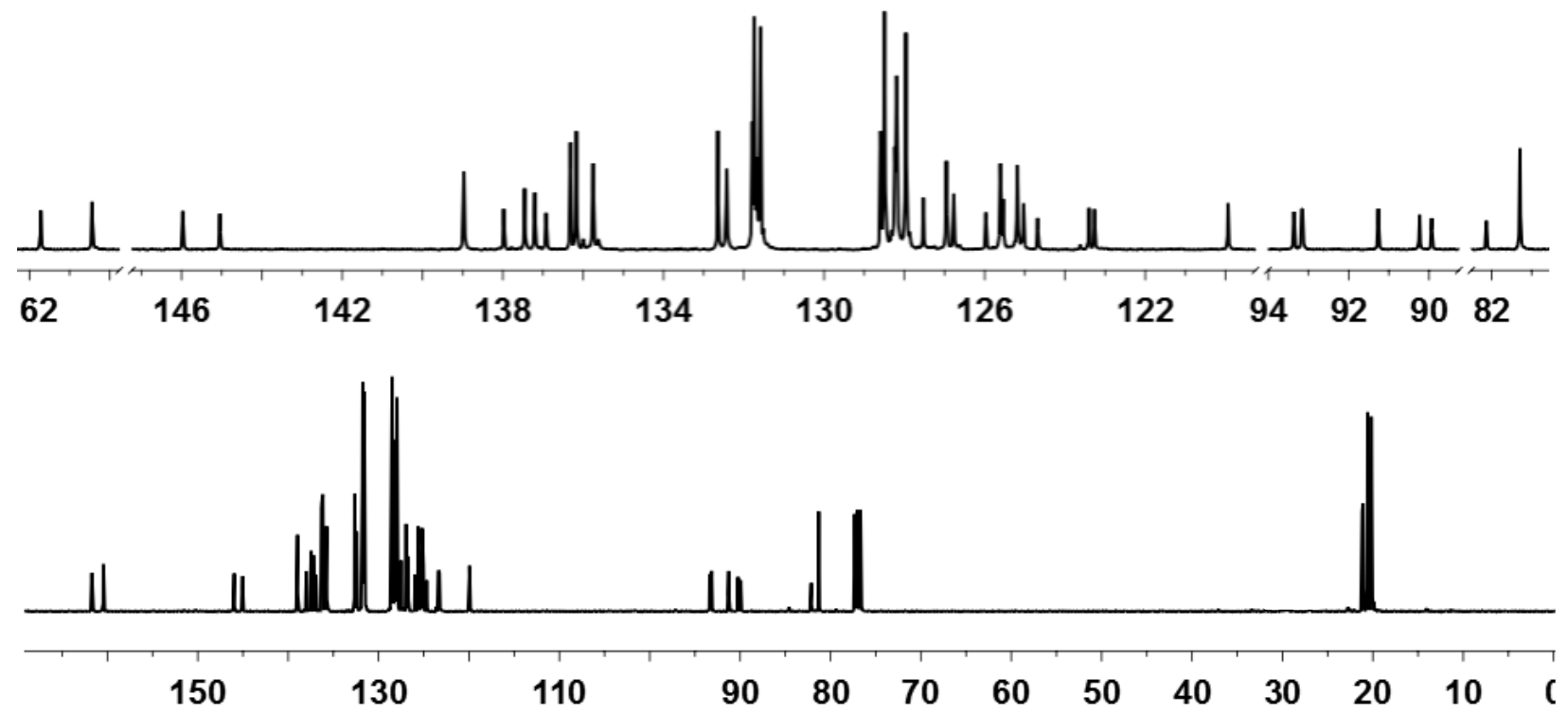

Figure S30: ${ }^{13} \mathrm{C}$ NMR spectrum $\left(\mathrm{CDCl}_{3}, 100 \mathrm{MHz}, 298 \mathrm{~K}\right)$ of ligand 23.
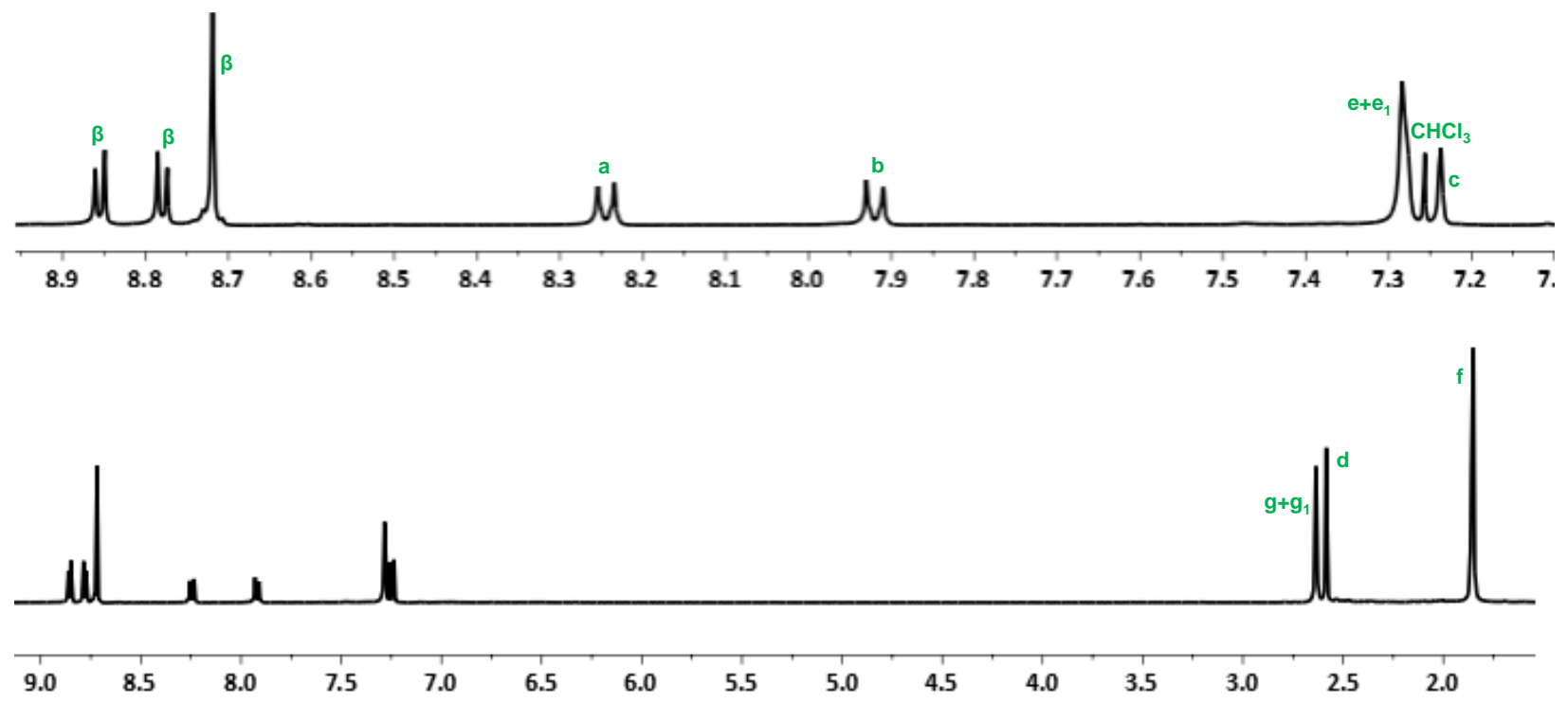

Figure S31: ${ }^{1} \mathrm{H}$ NMR spectrum $\left(\mathrm{CDCl}_{3}, 400 \mathrm{MHz}, 298 \mathrm{~K}\right)$ of rotator 2. 

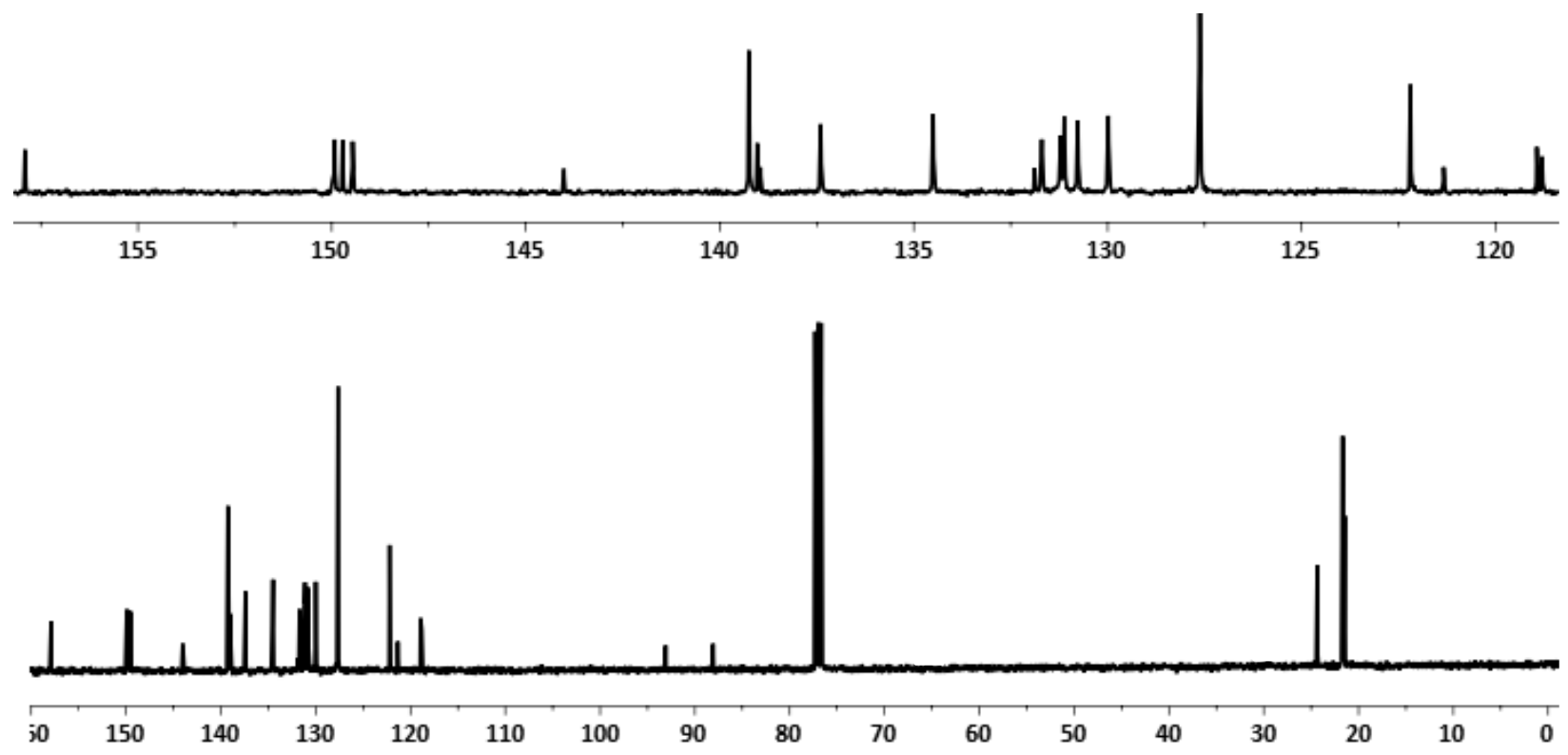

Figure S32: ${ }^{13} \mathrm{C}$ NMR spectrum $\left(\mathrm{CDCl}_{3}, 100 \mathrm{MHz}, 298 \mathrm{~K}\right)$ of rotator 2.
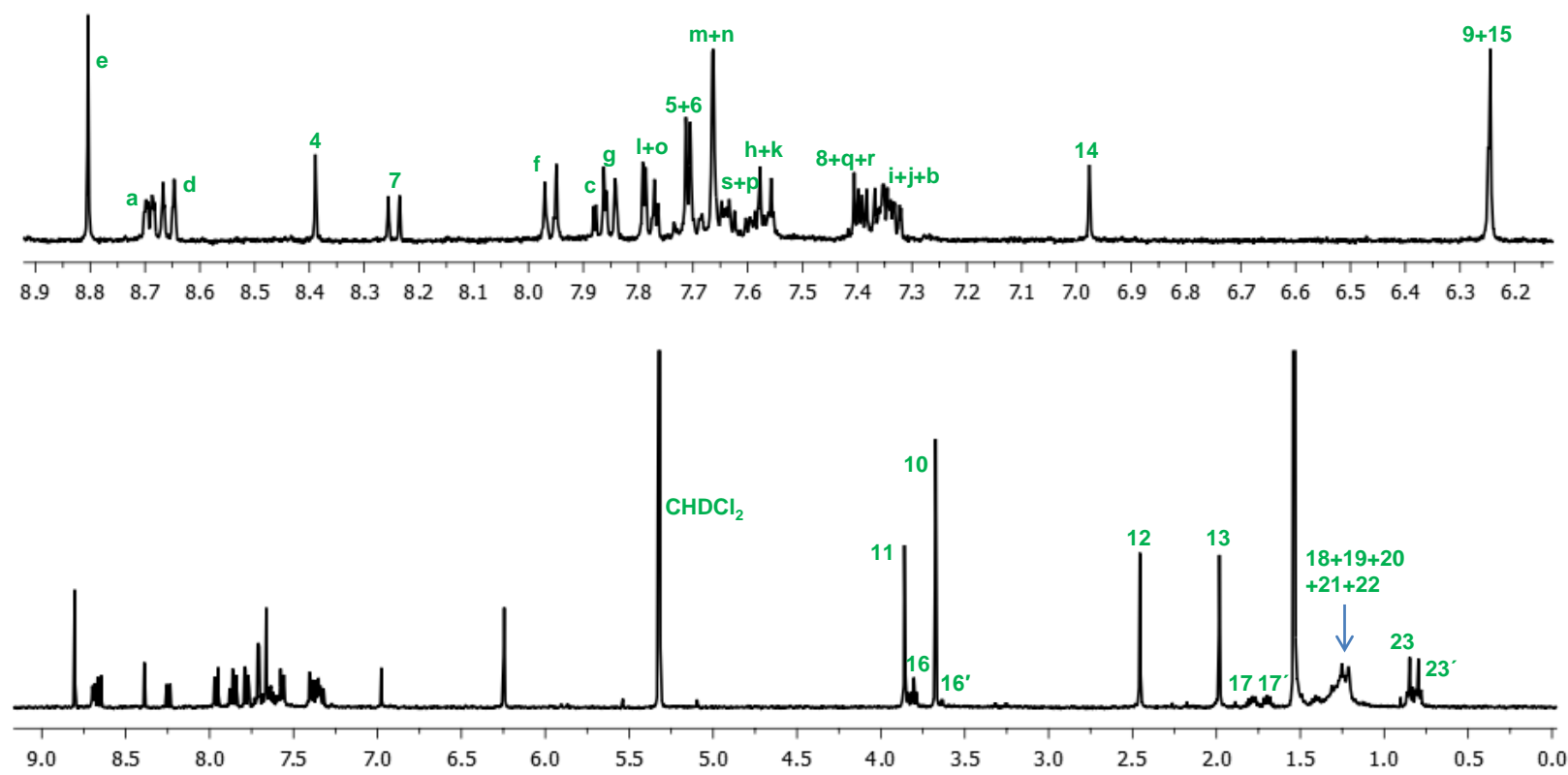

Figure S33: ${ }^{1} \mathrm{H}$ NMR spectrum $\left(\mathrm{CDCl}_{3}, 400 \mathrm{MHz}, 298 \mathrm{~K}\right)$ of nanoswitch 3. 


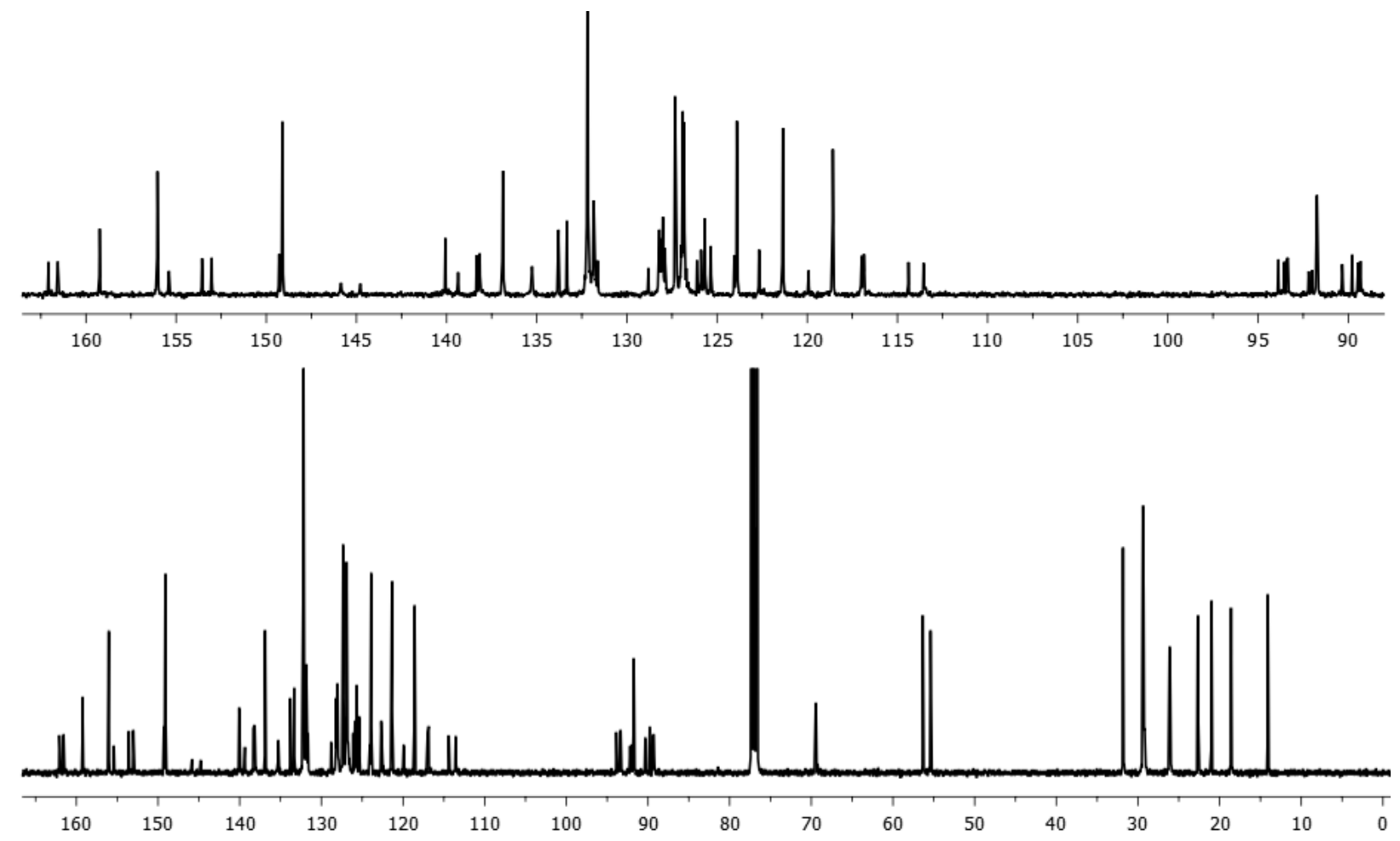

Figure S34: ${ }^{13} \mathrm{C}$ NMR spectrum $\left(\mathrm{CDCl}_{3}, 100 \mathrm{MHz}, 298 \mathrm{~K}\right)$ of nanoswitch 3.

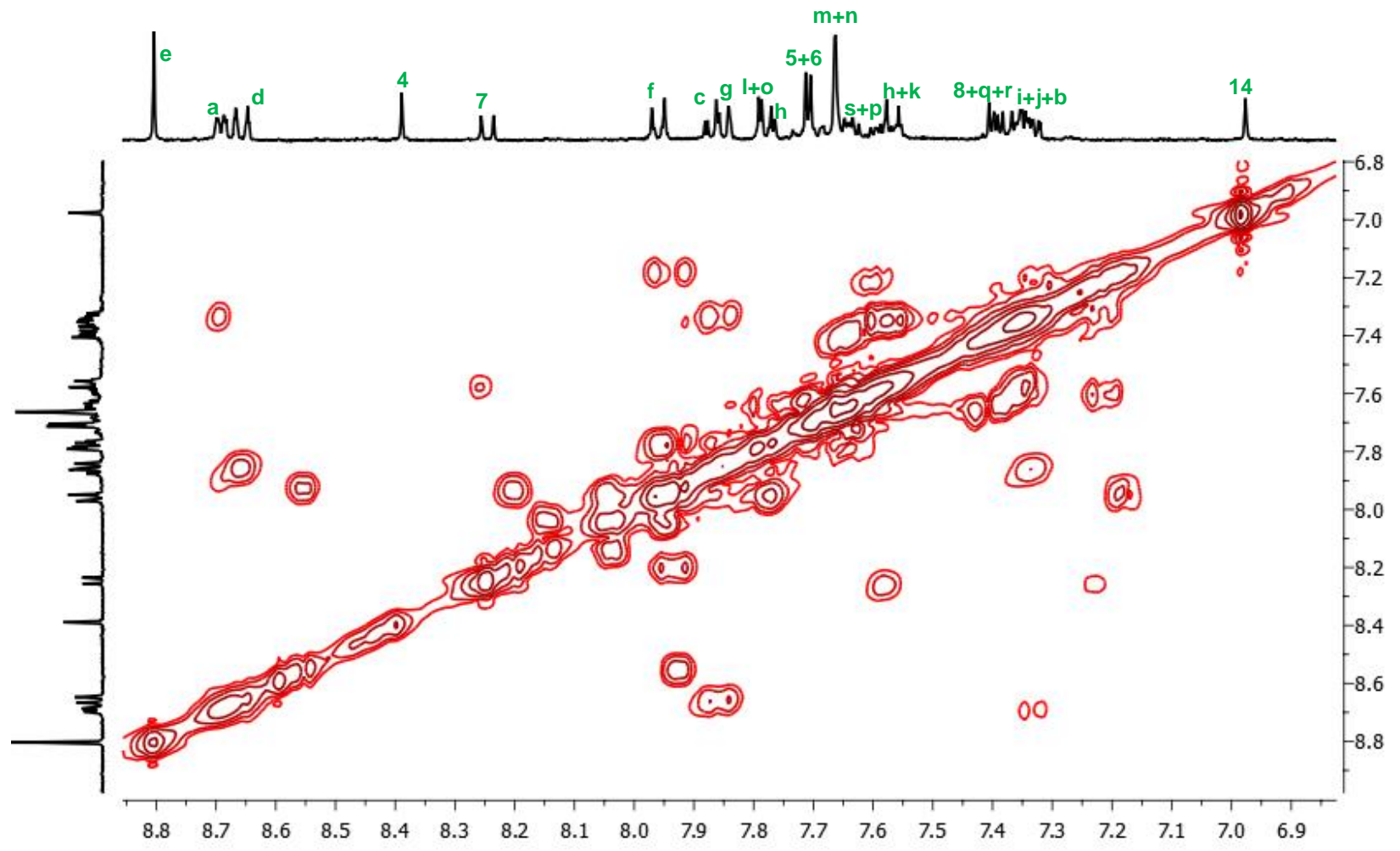

Figure S35: ${ }^{1} \mathrm{H}-{ }^{1} \mathrm{H}$ COSY NMR spectrum $\left(\mathrm{CD}_{2} \mathrm{Cl}_{2}, 400 \mathrm{MHz}, 298 \mathrm{~K}\right)$ of nanoswitch 3. 


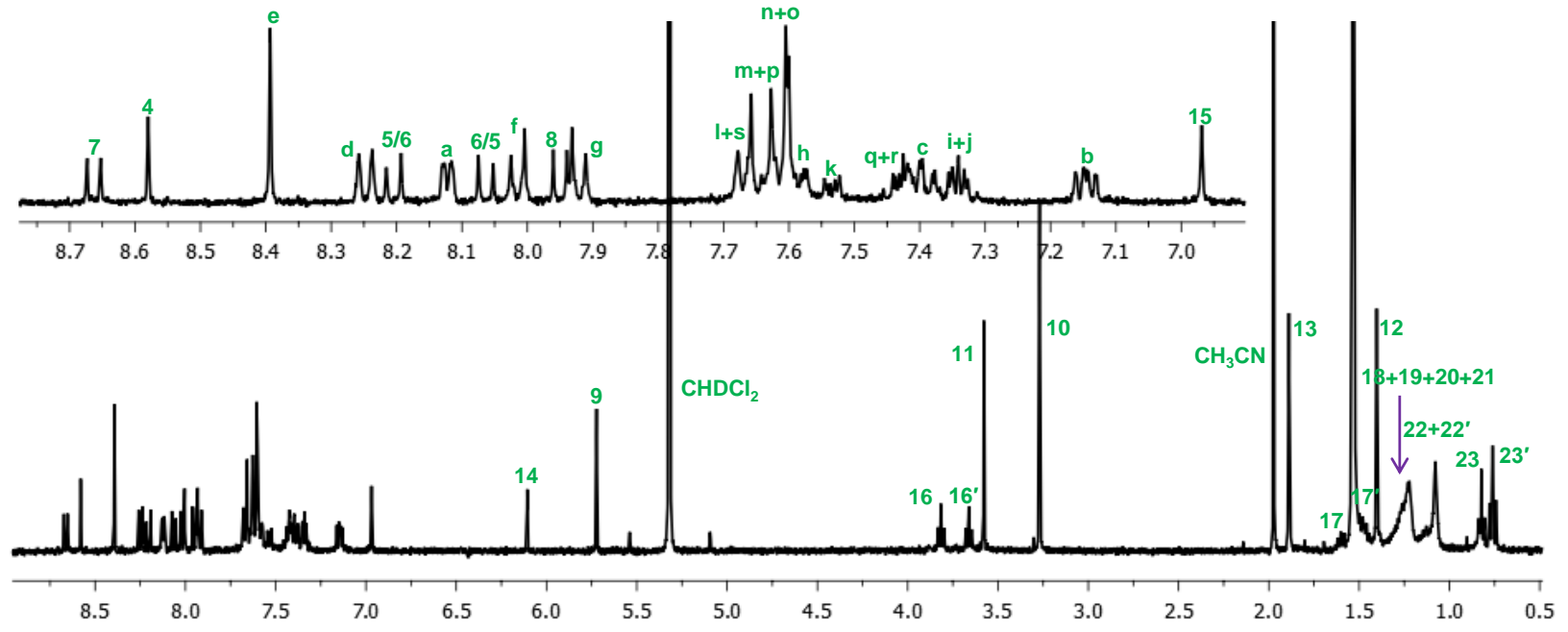

Figure S36: ${ }^{1} \mathrm{H}$ NMR spectrum $\left(\mathrm{CD}_{2} \mathrm{Cl}_{2}, 400 \mathrm{MHz}, 298 \mathrm{~K}\right)$ of $[\mathrm{Cu}(3)]^{+}$.

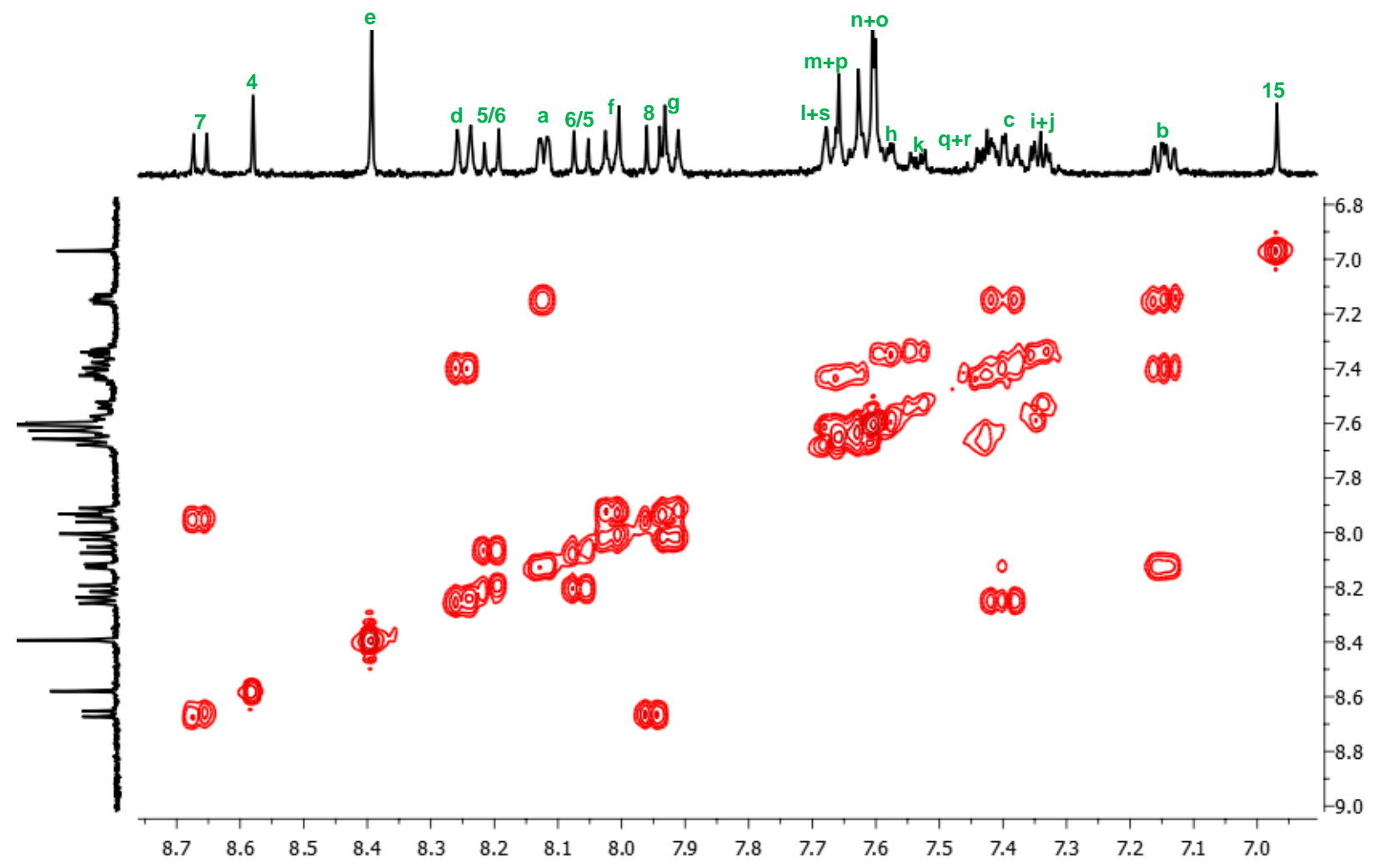

Figure S37: ${ }^{1} \mathrm{H}-{ }^{1} \mathrm{H}$ COSY NMR spectrum $\left(\mathrm{CD}_{2} \mathrm{Cl}_{2}, 400 \mathrm{MHz}, 298 \mathrm{~K}\right)$ of $[\mathrm{Cu}(\mathbf{3})]^{+}$. 


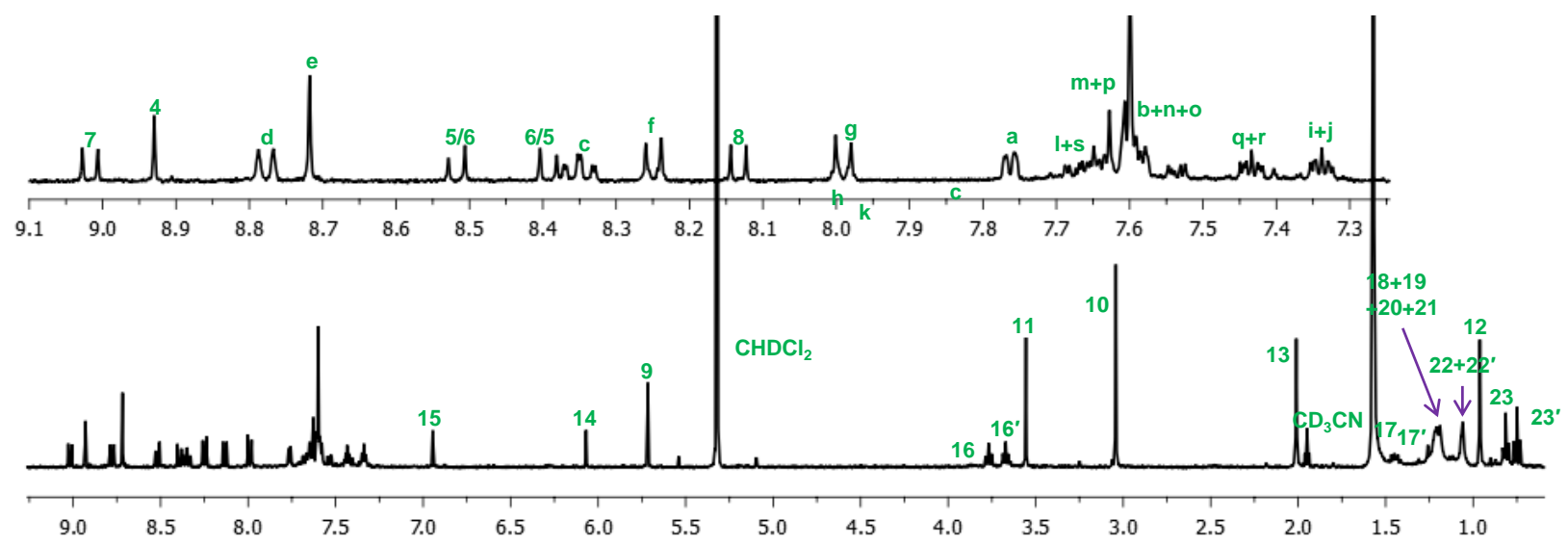

Figure S38: ${ }^{1} \mathrm{H}$ NMR spectrum $\left(\mathrm{CD}_{2} \mathrm{Cl}_{2}, 400 \mathrm{MHz}, 298 \mathrm{~K}\right)$ of $[\mathrm{Zn}(3)]^{2+}$.

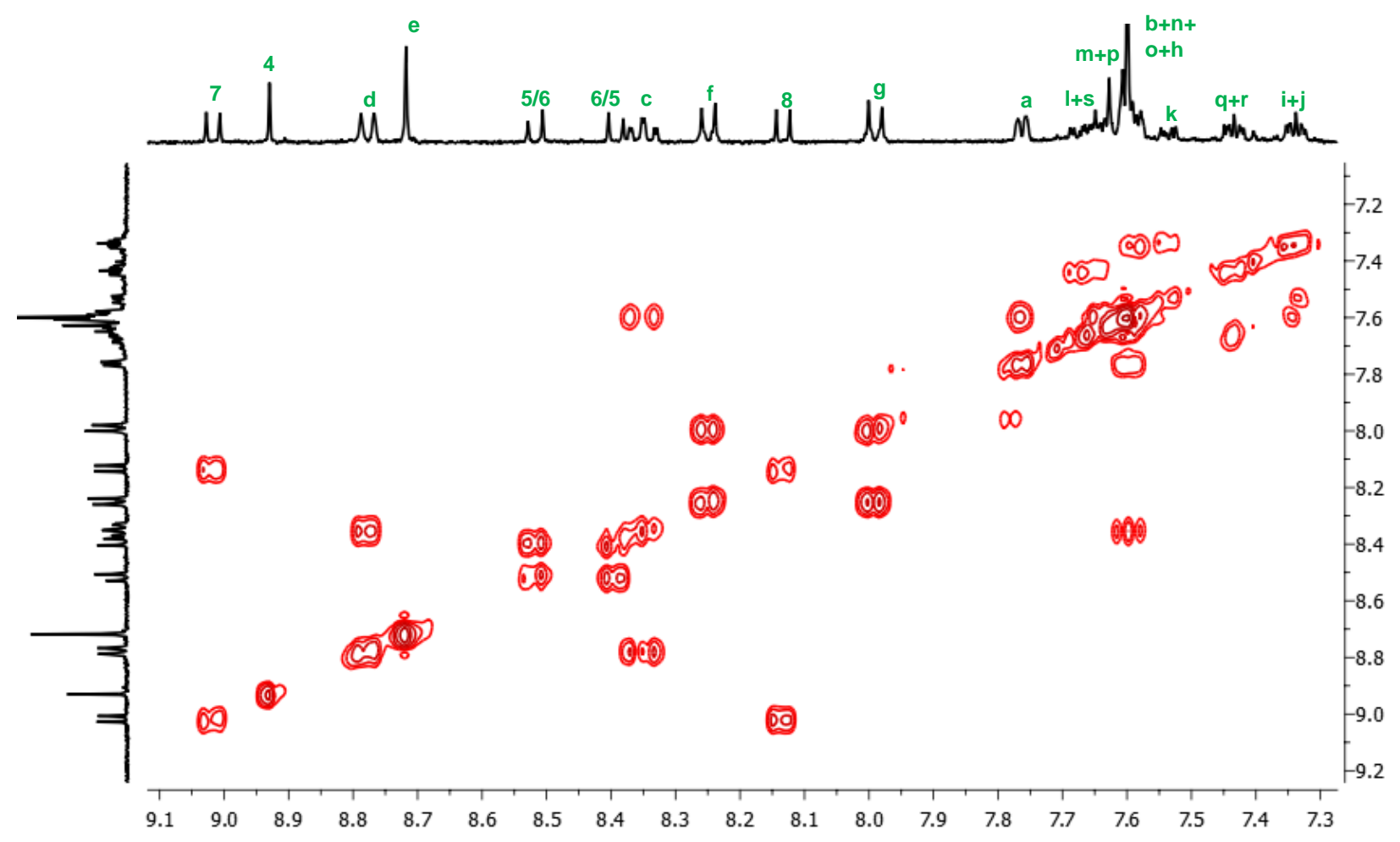

Figure S39: ${ }^{1} \mathrm{H}-{ }^{1} \mathrm{H}$ COSY NMR spectrum $\left(\mathrm{CD}_{2} \mathrm{Cl}_{2}, 400 \mathrm{MHz}, 298 \mathrm{~K}\right)$ of $[\mathrm{Zn}(3)]^{2+}$. 


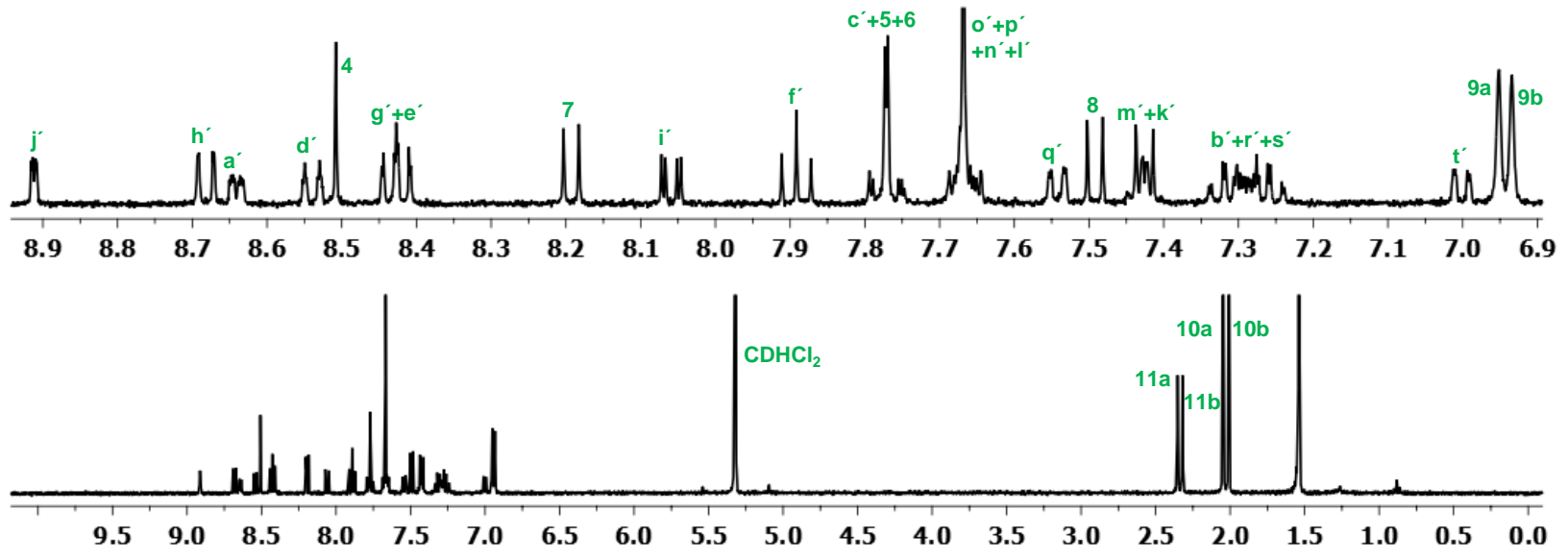

Figure S40: ${ }^{1} \mathrm{H}$ NMR spectrum $\left(\mathrm{CD}_{2} \mathrm{Cl}_{2}, 400 \mathrm{MHz}, 298 \mathrm{~K}\right)$ of nanoswitch 4.
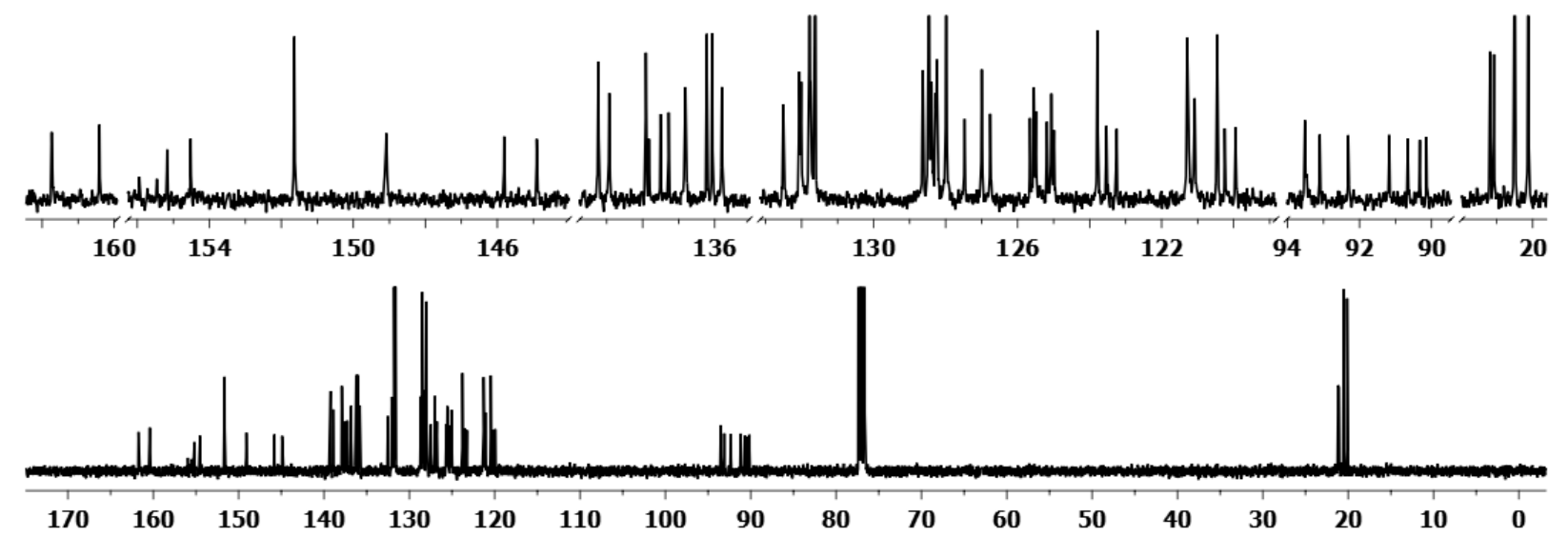

Figure S41: ${ }^{13} \mathrm{C}$ NMR spectrum $\left(\mathrm{CDCl}_{3}, 100 \mathrm{MHz}, 298 \mathrm{~K}\right)$ of nanoswitch 4. 


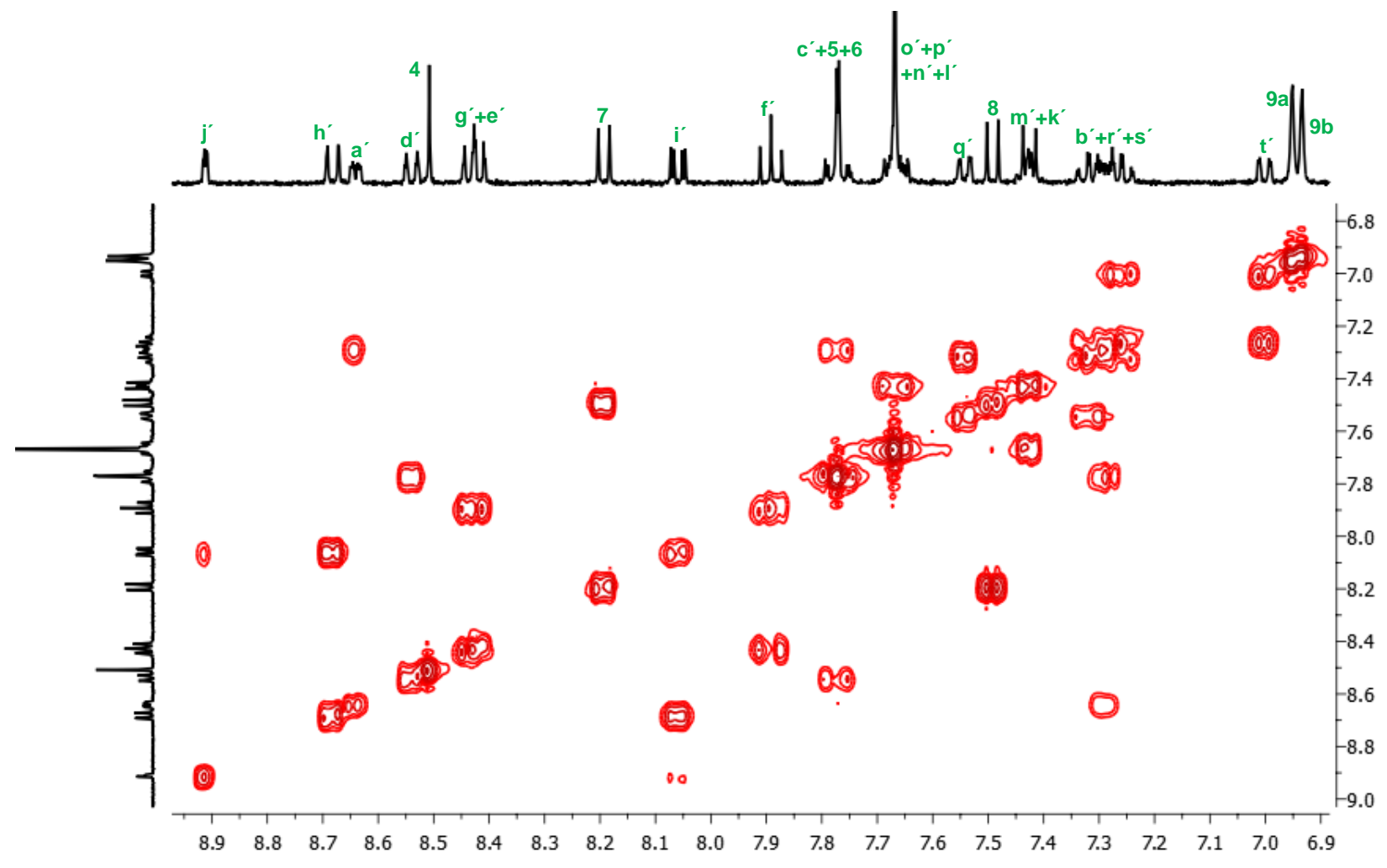

Figure S42: ${ }^{1} \mathrm{H}-{ }^{1} \mathrm{H}$ COSY NMR spectrum $\left(\mathrm{CD}_{2} \mathrm{Cl}_{2}, 400 \mathrm{MHz}, 298 \mathrm{~K}\right)$ of nanoswitch 4.

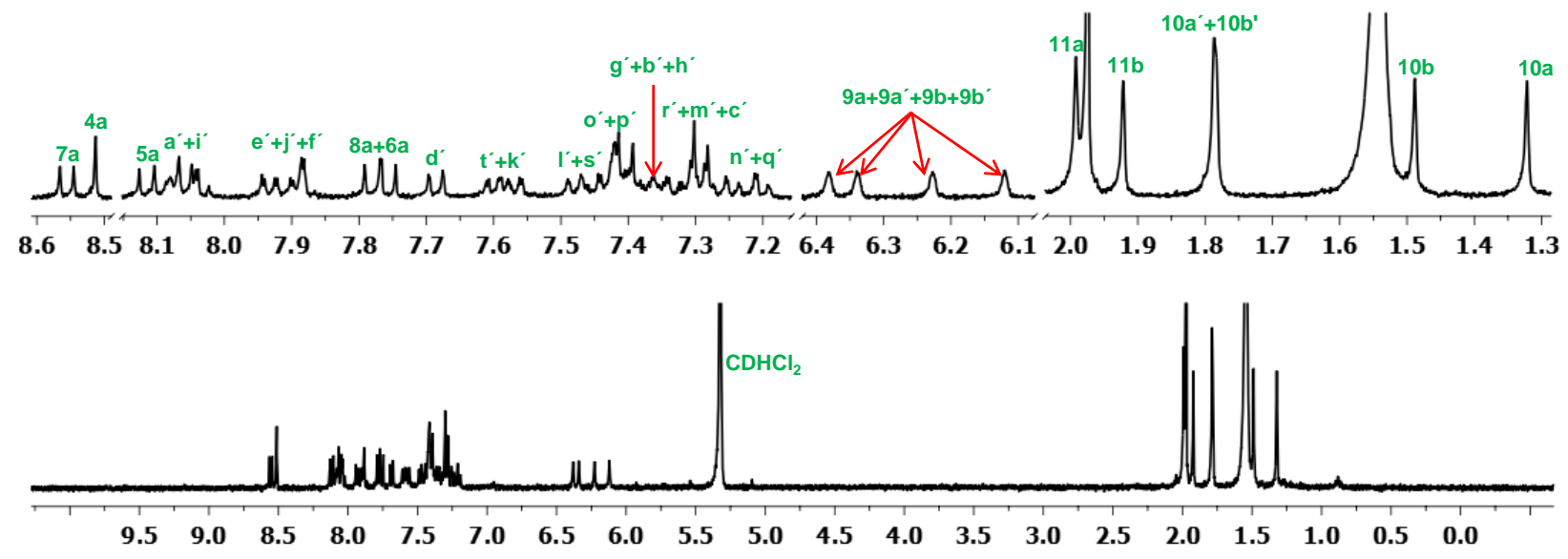

Figure S43: ${ }^{1} \mathrm{H}$ NMR spectrum $\left(\mathrm{CD}_{2} \mathrm{Cl}_{2}, 400 \mathrm{MHz}, 298 \mathrm{~K}\right)$ of $[\mathrm{Cu}(4)]^{+}$. 


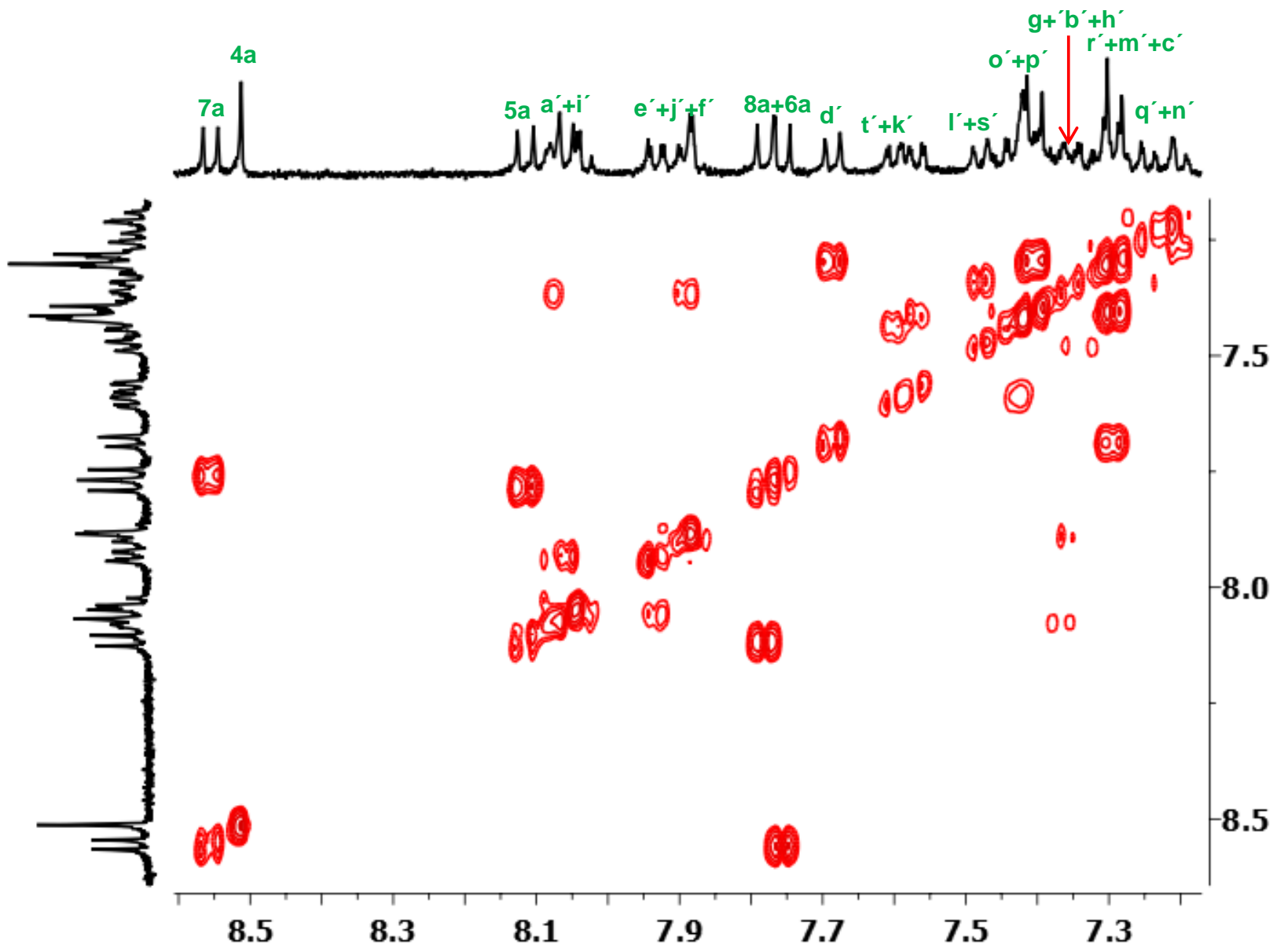

Figure S44: ${ }^{1} \mathrm{H}-{ }^{1} \mathrm{H}$ COSY NMR spectrum $\left(\mathrm{CD}_{2} \mathrm{Cl}_{2}, 400 \mathrm{MHz}, 298 \mathrm{~K}\right)$ of $[\mathrm{Cu}(4)]^{+}$.
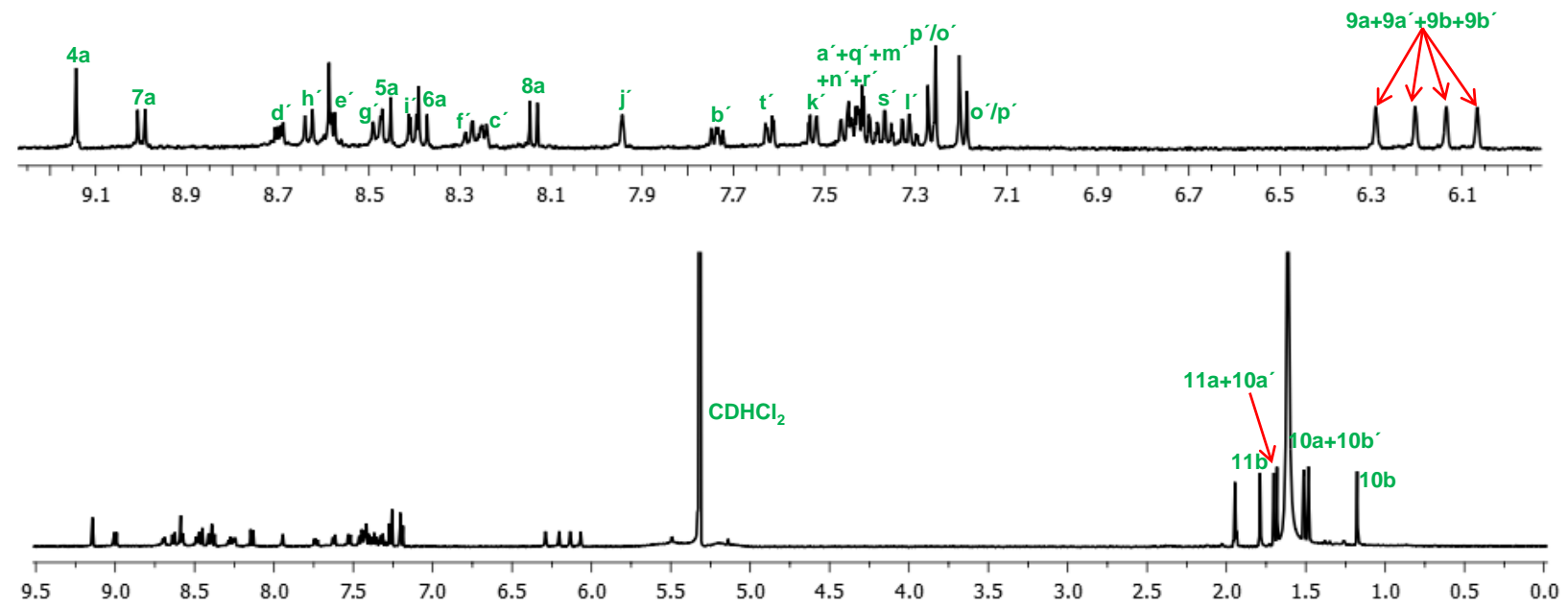

Figure S45: ${ }^{1} \mathrm{H}$ NMR spectrum $\left(\mathrm{CD}_{2} \mathrm{Cl}_{2}, 500 \mathrm{MHz}, 298 \mathrm{~K}\right)$ of $[\mathrm{Hg}(4)]^{2+}$. 


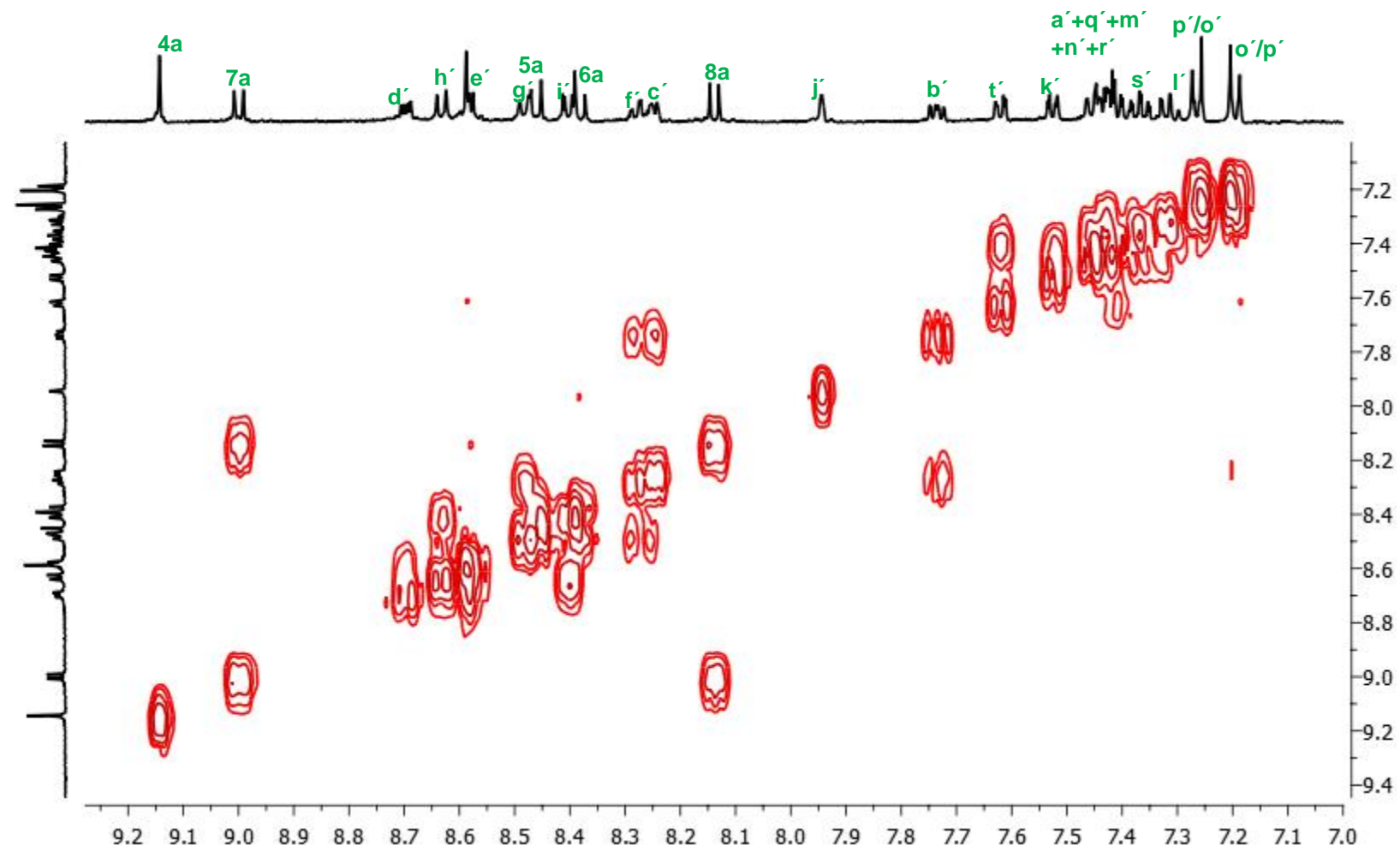

Figure S46: ${ }^{1} \mathrm{H}-{ }^{1} \mathrm{H}$ COSY NMR spectrum $\left(\mathrm{CD}_{2} \mathrm{Cl}_{2}, 500 \mathrm{MHz}, 298 \mathrm{~K}\right)$ of $[\mathrm{Hg}(4)]^{2+}$. 

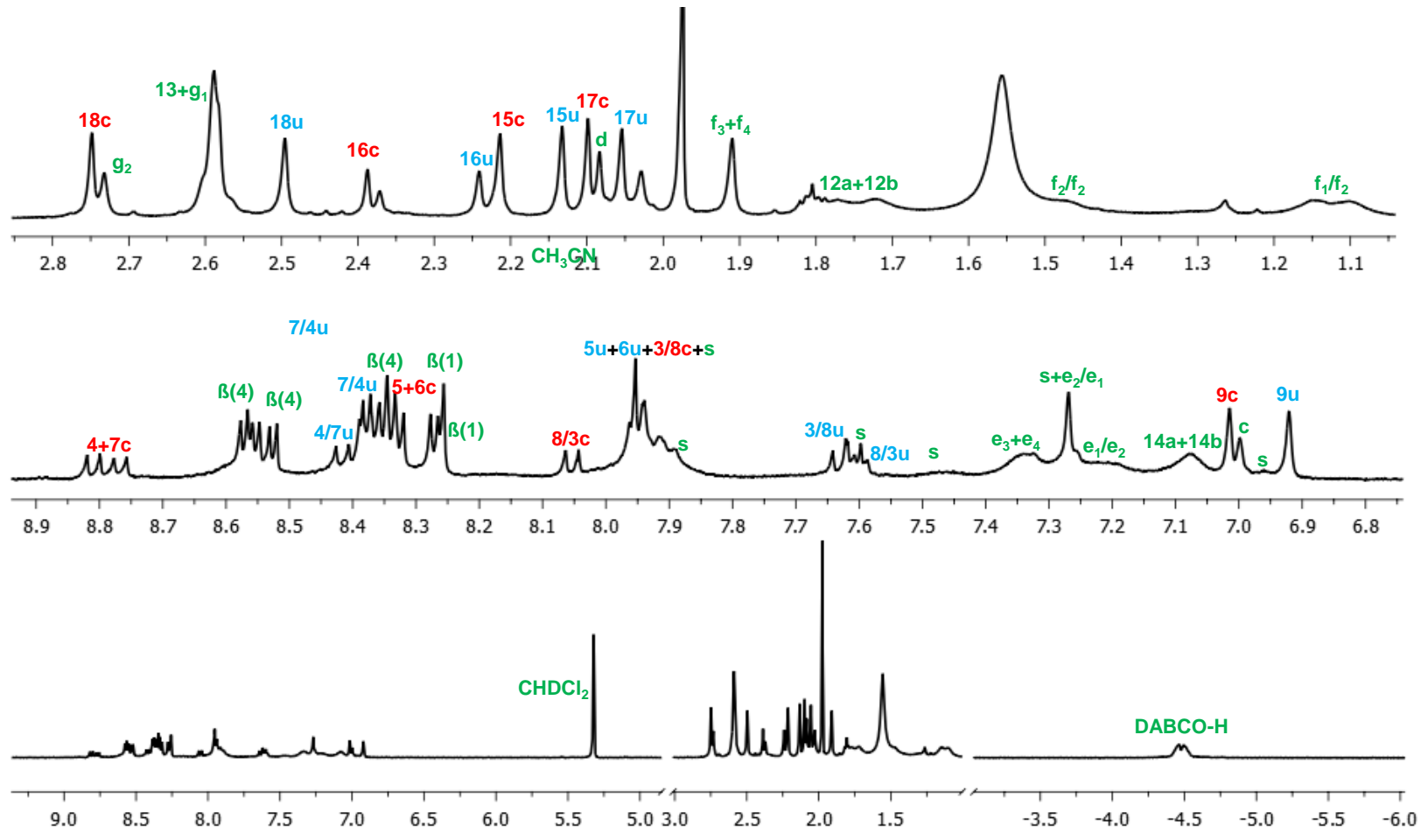

Figure S47: ${ }^{1} \mathrm{H}$ NMR spectrum $\left(\mathrm{CD}_{2} \mathrm{Cl}_{2}, 400 \mathrm{MHz}, 298 \mathrm{~K}\right)$ of ROT-1.

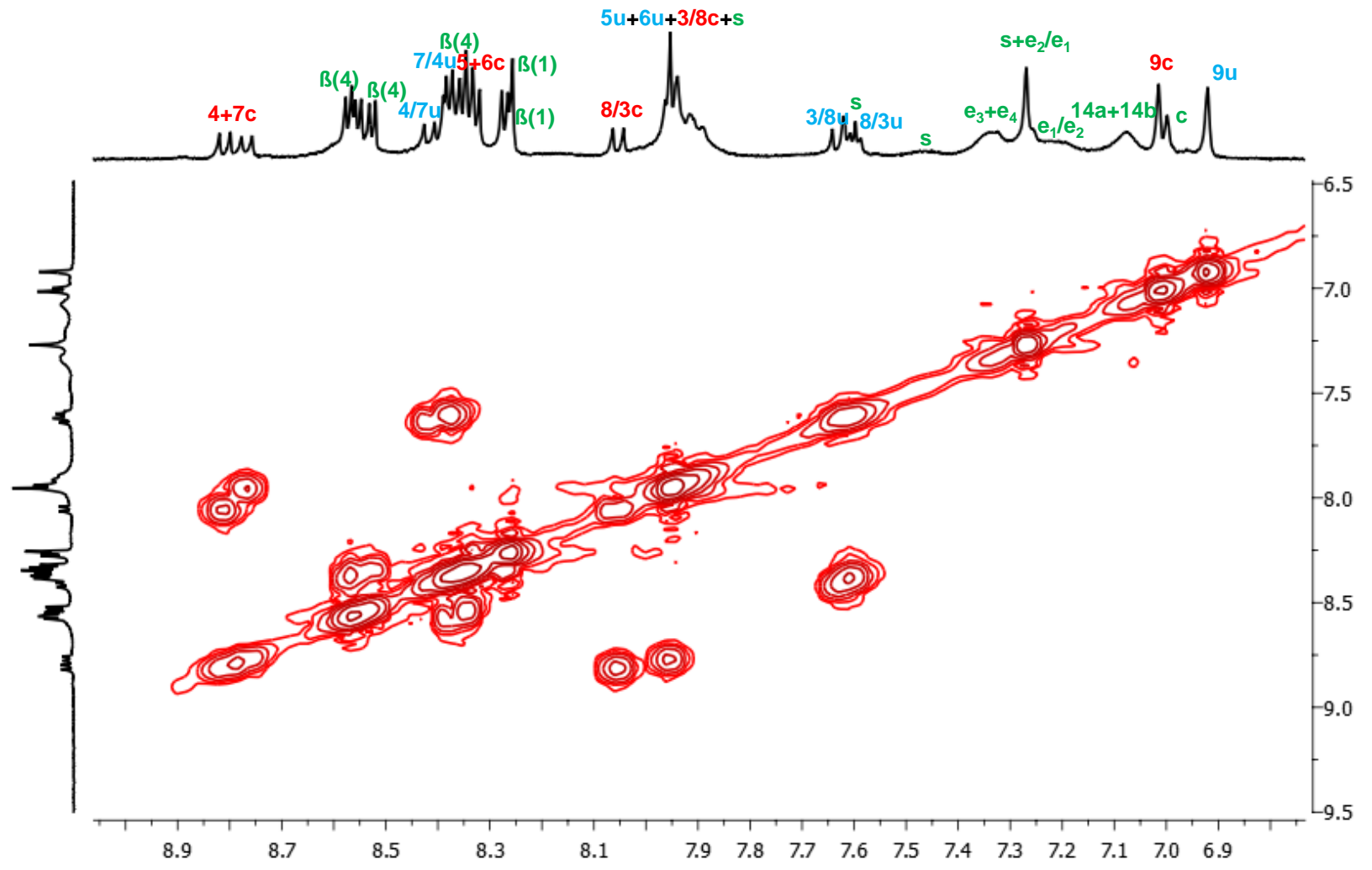

Figure S48: ${ }^{1} \mathrm{H}-{ }^{1} \mathrm{H}$ COSY NMR spectrum $\left(\mathrm{CD}_{2} \mathrm{Cl}_{2}, 400 \mathrm{MHz}, 298 \mathrm{~K}\right)$ of ROT-1. 


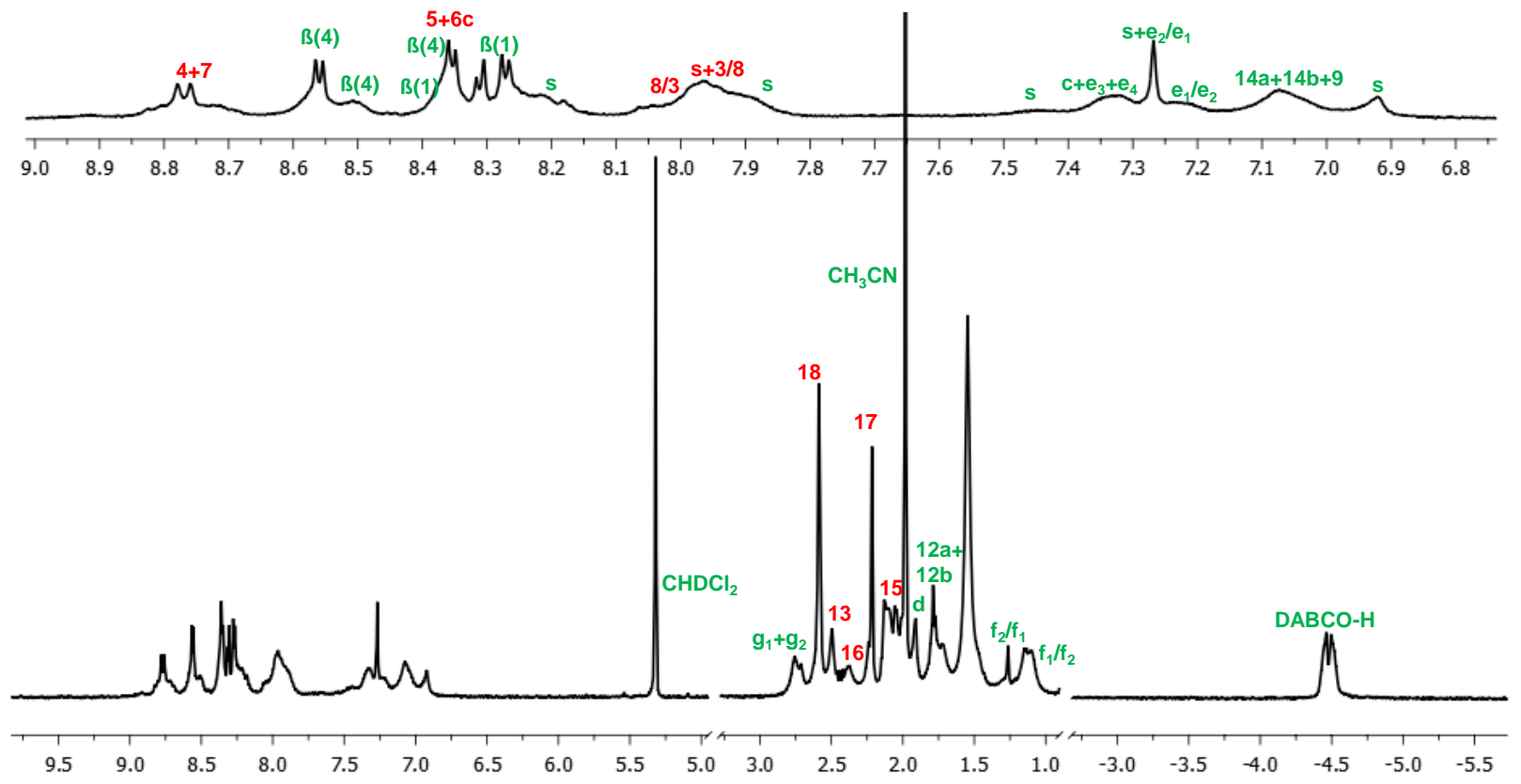

Figure S49: ${ }^{1} \mathrm{H}$ NMR spectrum $\left(\mathrm{CD}_{2} \mathrm{Cl}_{2}, 400 \mathrm{MHz}, 298 \mathrm{~K}\right)$ of ROT-2.

(c) $[\mathrm{Zn}(\mathbf{3})]^{2+}$

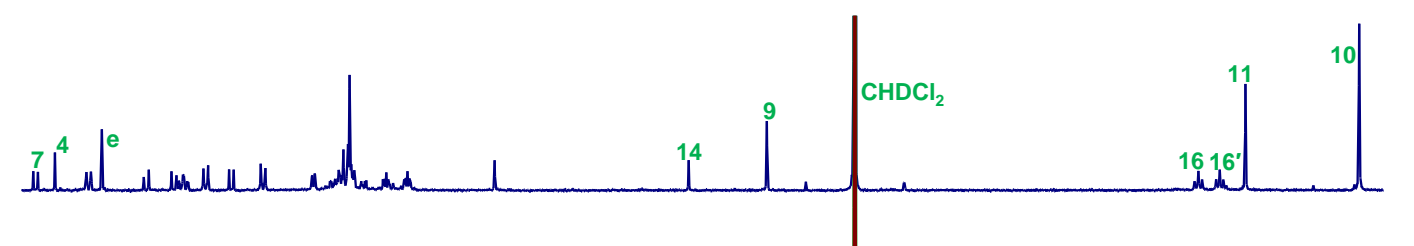

(b) $[\mathrm{Zn}(\mathbf{3})]^{2+}+$ free $\mathrm{Cu}+$

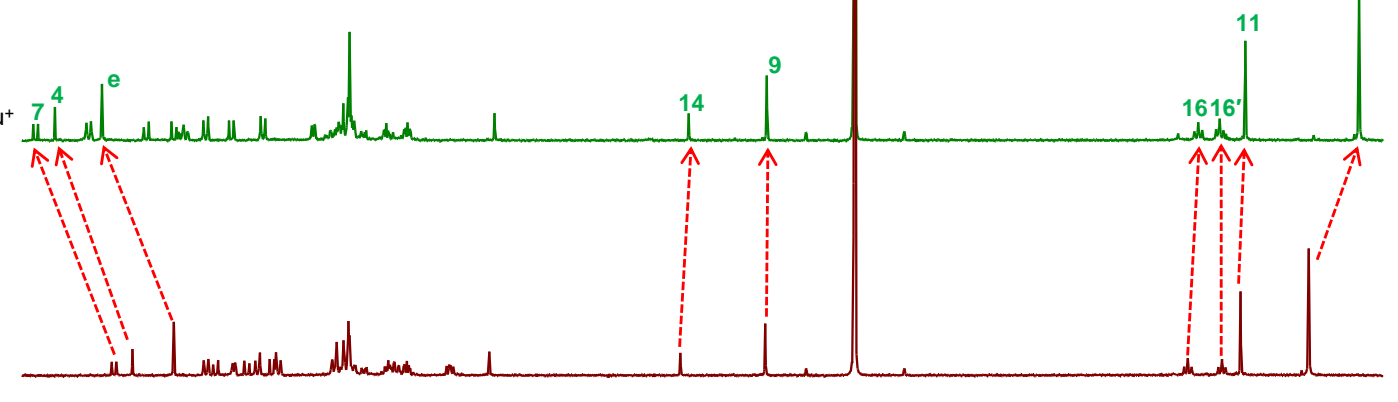

(a) $[\mathrm{Cu}(3)]^{+}$
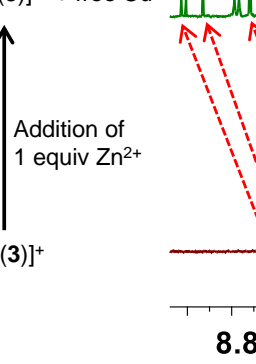

7.8

6.8

5.8

4.8

3.8

Figure S50. Partial ${ }^{1} \mathrm{H}$ NMR $\left(\mathrm{CD}_{2} \mathrm{Cl}_{2}, 400 \mathrm{MHz}, 298 \mathrm{~K}\right)$ of (a) $[\mathrm{Cu}(3)]^{+}$, (b) $[\mathrm{Cu}(3)]^{+}$upon addition of 1 equiv of $\mathrm{Zn}^{2+}$ after $3 \mathrm{~h}$, (c) $[\mathrm{Zn}(\mathbf{3})]^{2+}$. 


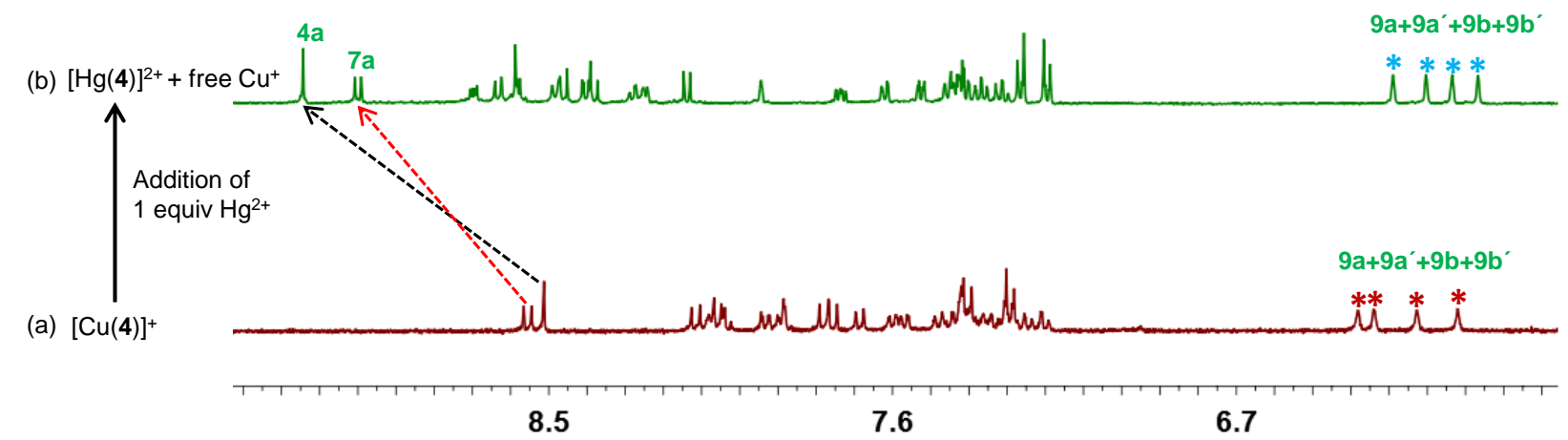

Figure S51: Partial ${ }^{1} \mathrm{H}$ NMR $\left(\mathrm{CD}_{2} \mathrm{Cl}_{2}, 400 \mathrm{MHz}, 298 \mathrm{~K}\right)$ of (a) $[\mathrm{Cu}(4)]^{+}$, (b) $[\mathrm{Cu}(4)]^{+}$upon addition of 1 equiv of $\mathrm{Hg}^{2+}$ (c) $[\mathrm{Hg}(4)]^{2+}$.

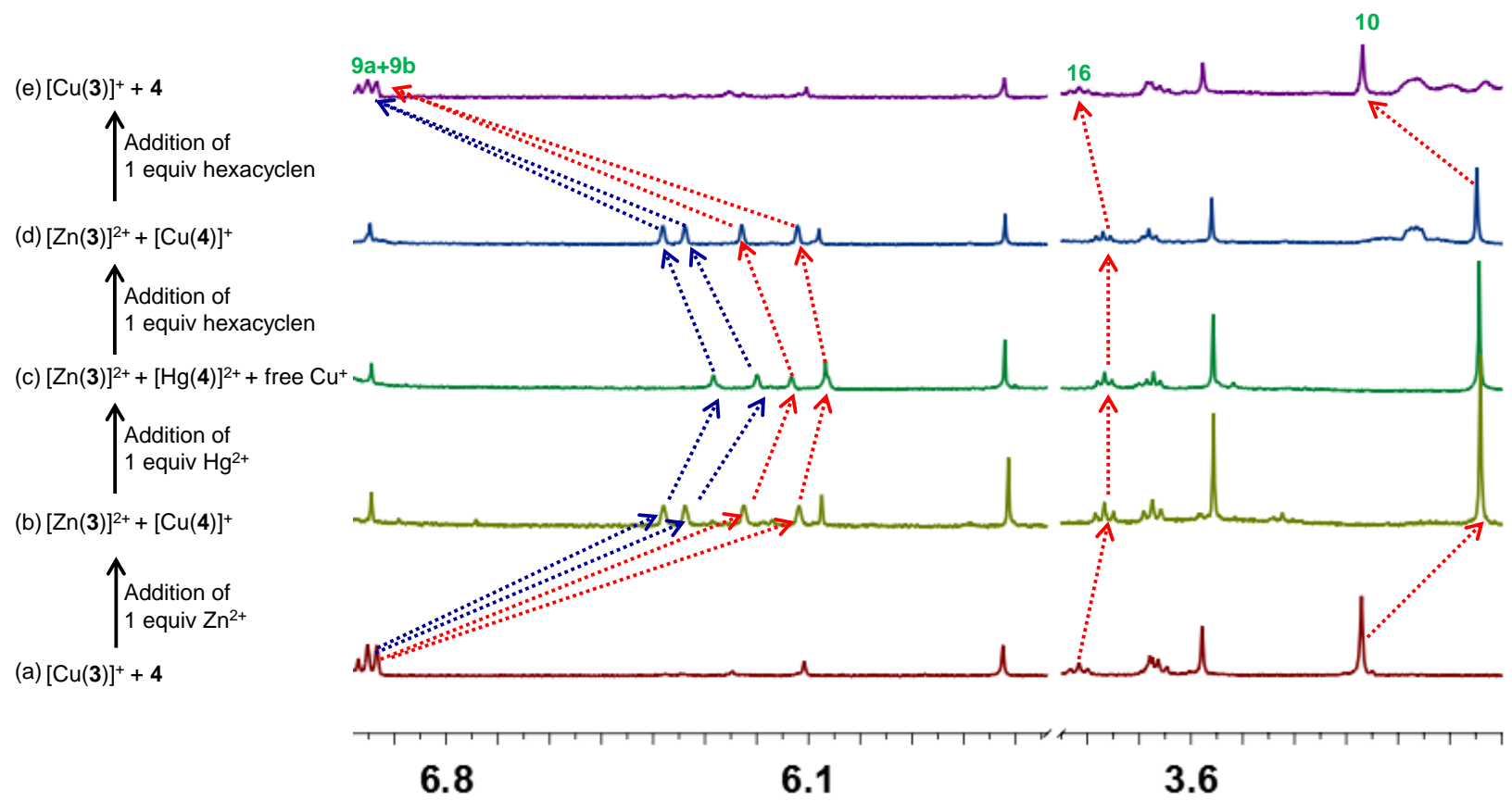

Figure S52: Partial ${ }^{1} \mathrm{H}$ NMR $\left(\mathrm{CD}_{2} \mathrm{Cl}_{2}, 500 \mathrm{MHz}, 298 \mathrm{~K}\right)$ of (a) $[\mathrm{Cu}(3)]^{+}+4$, (b) upon addition of 1 equiv of $\mathrm{Zn}^{2+}$ to (a) after $3 \mathrm{~h}$, (c) upon addition of 1 equiv of $\mathrm{Hg}^{2+}$ to (b), (d) after addition of 1 equiv of hexacyclen to (c), (e) after addition of 1 equiv of hexacyclen to (d). 


\section{DOSY NMR Spectra}

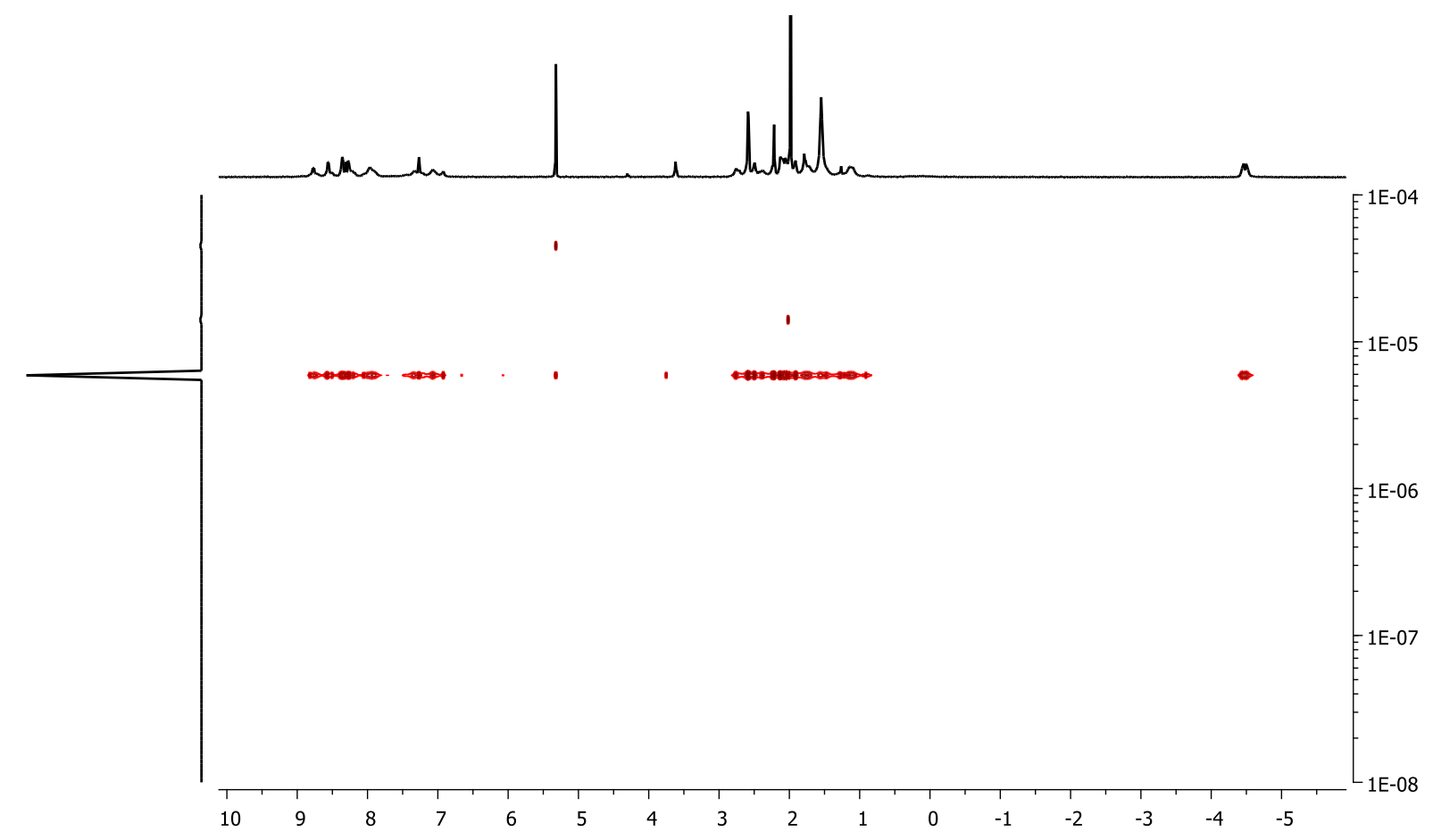

Figure S53: ${ }^{1} \mathrm{H}-\mathrm{DOSY}$ NMR spectrum $\left(\mathrm{CD}_{2} \mathrm{Cl}_{2}, 400 \mathrm{MHz}, 298 \mathrm{~K}\right)$ of ROT-2. Diffusion coefficient $D=4.3 \times 10^{-10} \mathrm{~m}^{2} \mathrm{~s}^{-1}$, hydrodynamic radius $r=10.3 \AA$.

\section{VT ${ }^{1}$ H-NMR Spectra}

(a)

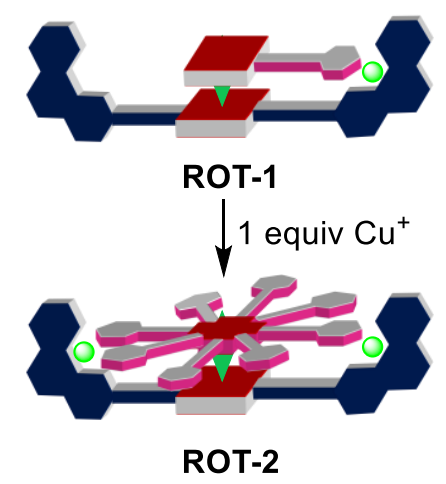

(b)
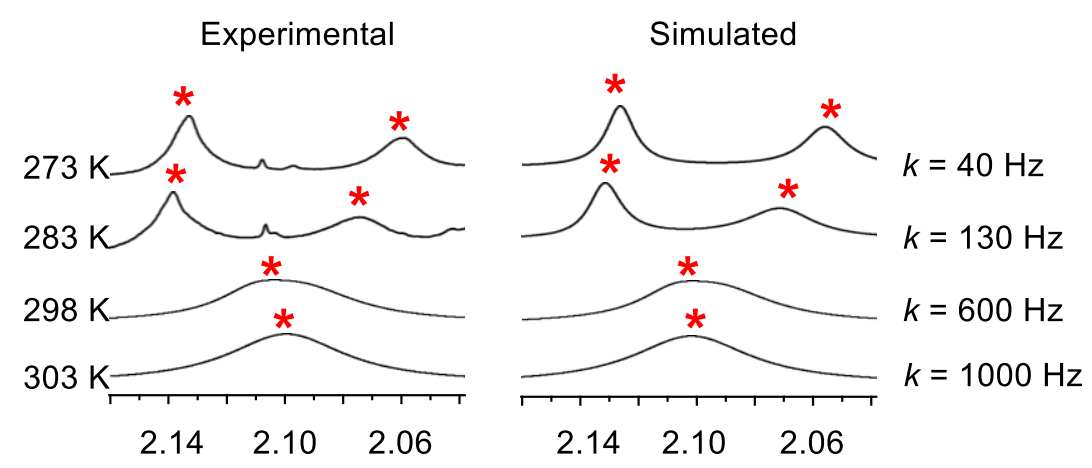

Figure S54: (a) Cartoon structure of ROT-1 and ROT-2. (b) Partial VT- ${ }^{1} \mathrm{H}$ NMR $\left(\mathrm{CD}_{2} \mathrm{Cl}_{2}, 600\right.$ $\mathrm{MHz}$ ) of ROT-2 showing the splitting of 15-H (red asterisk marked). 


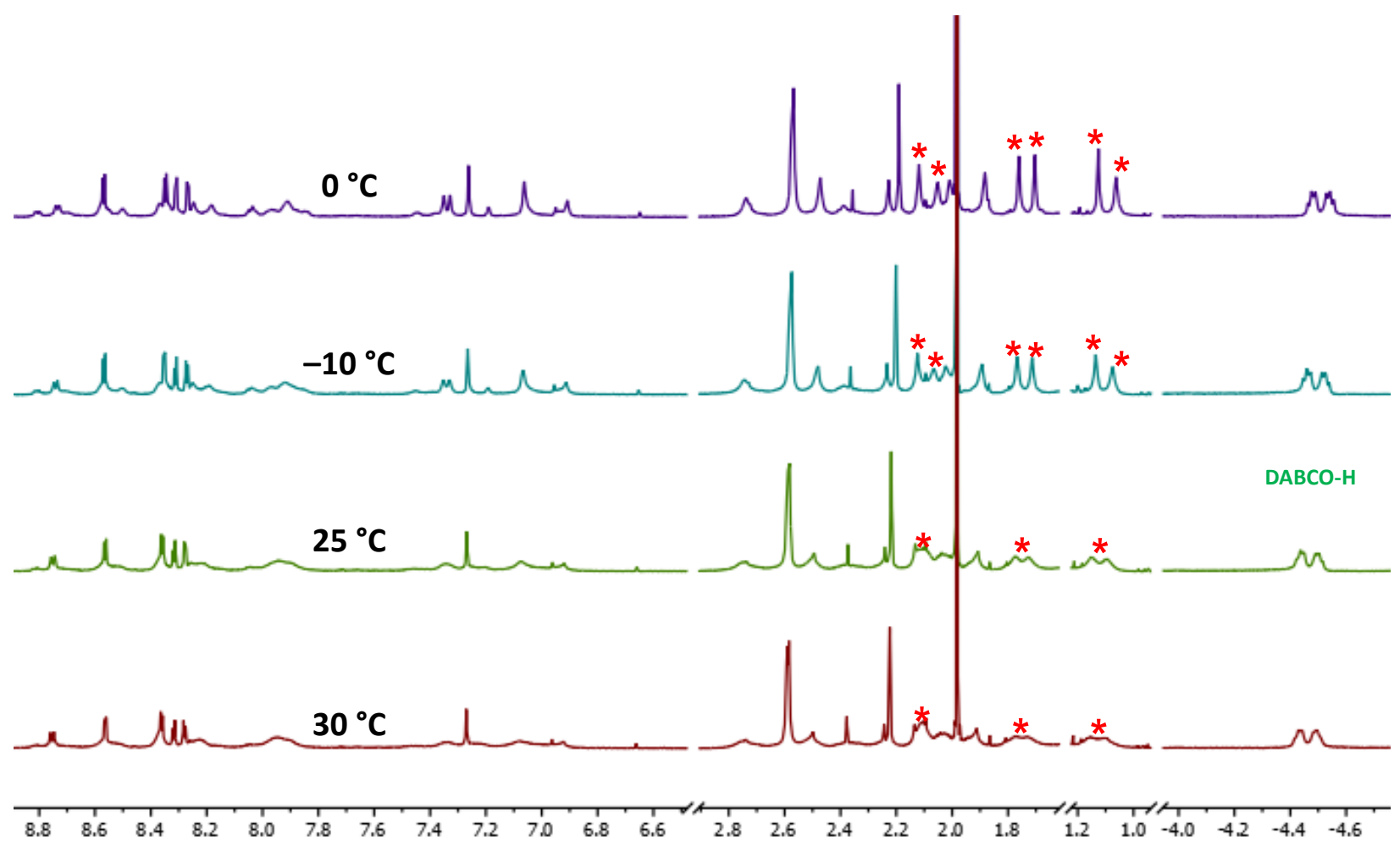

Figure S55: VT ${ }^{1} \mathrm{H}-\mathrm{NMR}$ spectrum of ROT-2 (600 MHz, $\left.\mathrm{CD}_{2} \mathrm{Cl}_{2}\right)$ showing the splitting of protons $15-\mathrm{H},\left[\mathrm{f}_{1} / \mathrm{f}_{2}\right]-\mathrm{H}$ and $[12 \mathrm{a} / 12 \mathrm{~b}]-\mathrm{H}$ (red asterisk marked). Characteristic signals for DABCO-H corroborate the intactness of the assembly throughout the temperature range.

\section{NMR Simulation Analysis to Determine the Rotational Frequency}

NMR simulation. Using a conventional dynamic NMR spectroscopic method, ${ }^{7}$ a model involving a two-spin system undergoing mutual exchange was applied to simulate the spectra and determine the exchange frequency. The NMR signal used for the simulation is indicated in the corresponding spectra by *. The exchange frequency in the rotor is identical with the rotational frequency and was obtained from an analysis of the exchange-broadened NMR of proton 16-H of the stator. Activation enthalpy $\left(\Delta H^{\ddagger}\right)$ and activation entropy $\left(\Delta S^{\ddagger}\right)$ were determined from transition state theory.

$k=\left(\mathrm{k}_{\mathrm{B}} \mathrm{T} / \mathrm{h}\right) \mathrm{e}^{-\Delta G \sharp / \mathrm{RT}}$

$\ln (k / \mathrm{T})=-\Delta H^{\ddagger} / \mathrm{RT}+\ln \left(\mathrm{k}_{\mathrm{B}} / \mathrm{h}\right)+\Delta S^{\ddagger} / \mathrm{R}$, where $\mathrm{R}=$ universal gas constant.

The free energy barrier at $298 \mathrm{~K}$ was determined using the equation $\Delta G^{\star} 298=\Delta H^{\star}-298 \mathrm{~T} \Delta S^{\star}$. 
The temperature dependency of the rotational motion was fitted to the Eyring ${ }^{8}$ equation:

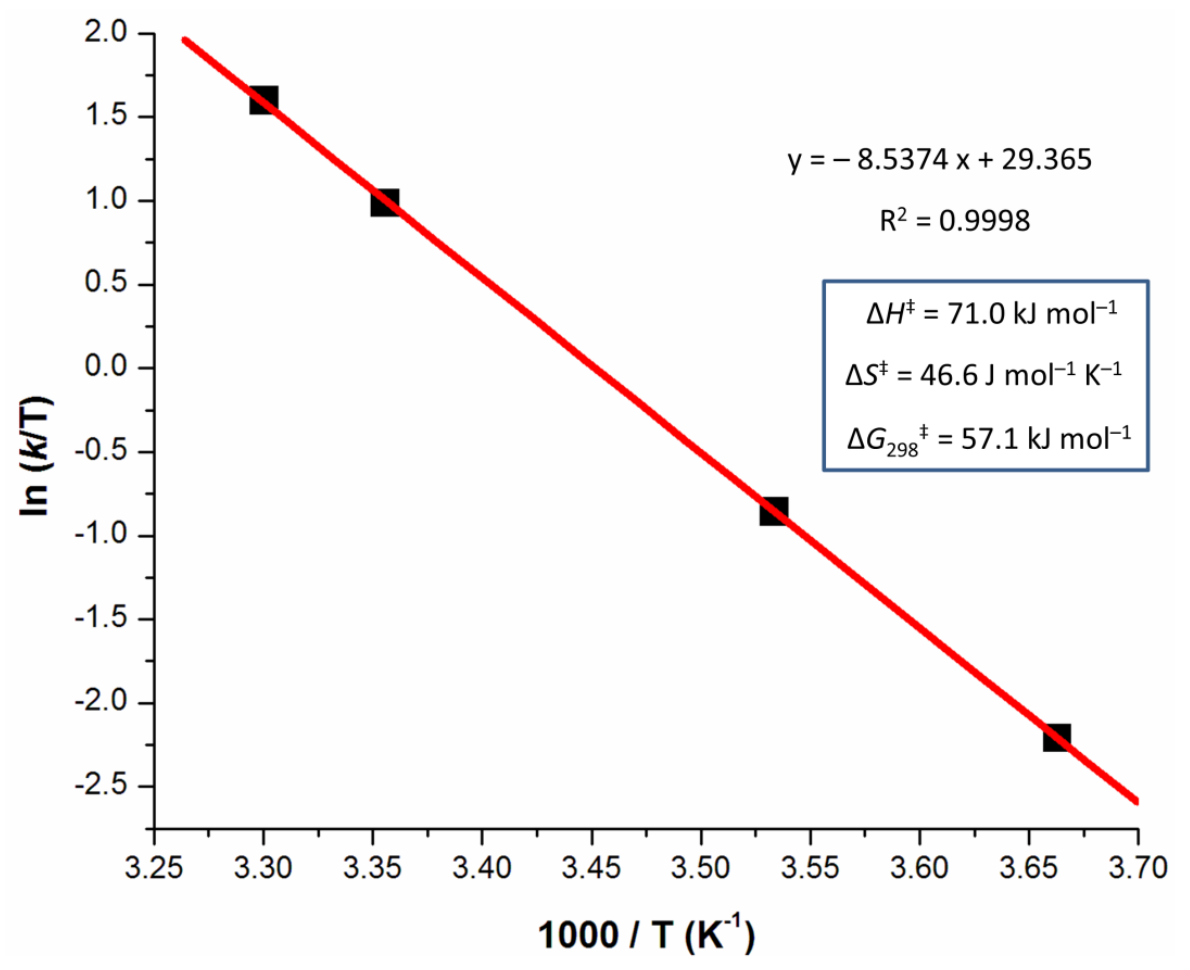

Figure S56: Eyring plot for the rotational dynamics in ROT-2.

\section{Catalytic Experiments}

\section{a) Rotors as catalyst}<smiles>C#Cc1ccc(N(C)C)cc1</smiles>

5<smiles>N#Cc1ccccc1N</smiles>

6

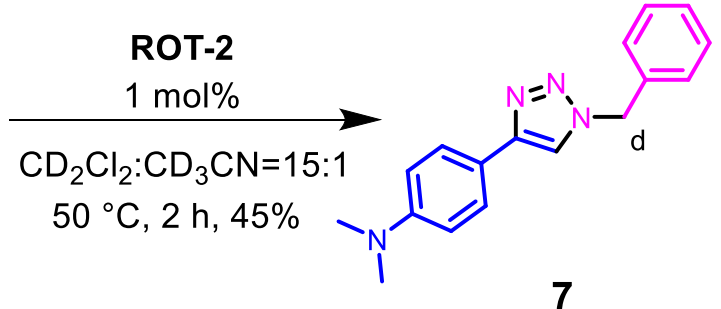

7

In an NMR tube, substrates $5(3.72 \mathrm{mg}, 25.6 \mu \mathrm{mol})$ and $\mathbf{6}(3.41 \mathrm{mg}, 25.6 \mu \mathrm{mol})$ were taken. Then 0.01 equiv of ROT-2 was used as a catalyst for the click reaction. After $2 \mathrm{~h}$ at $50{ }^{\circ} \mathrm{C}$ in $\mathrm{CD}_{2} \mathrm{Cl}_{2}: \mathrm{CD}_{3} \mathrm{CN}=15: 1$ (total $500 \mu \mathrm{L}$ ) the yield of click product $7(\mathrm{~d}-\mathrm{H}$ at $\delta=5.52 \mathrm{ppm}$ ) was 
determined using 1,3,5-trimethoxybenzene as internal standard. Integration of proton $\mathrm{d}-\mathrm{H}$ was monitored with respect to the aryl protons of trimethoxybenzene $(\delta=6.06 \mathrm{ppm})$ for yield calculation showing formation of $45 \%$ of 7.

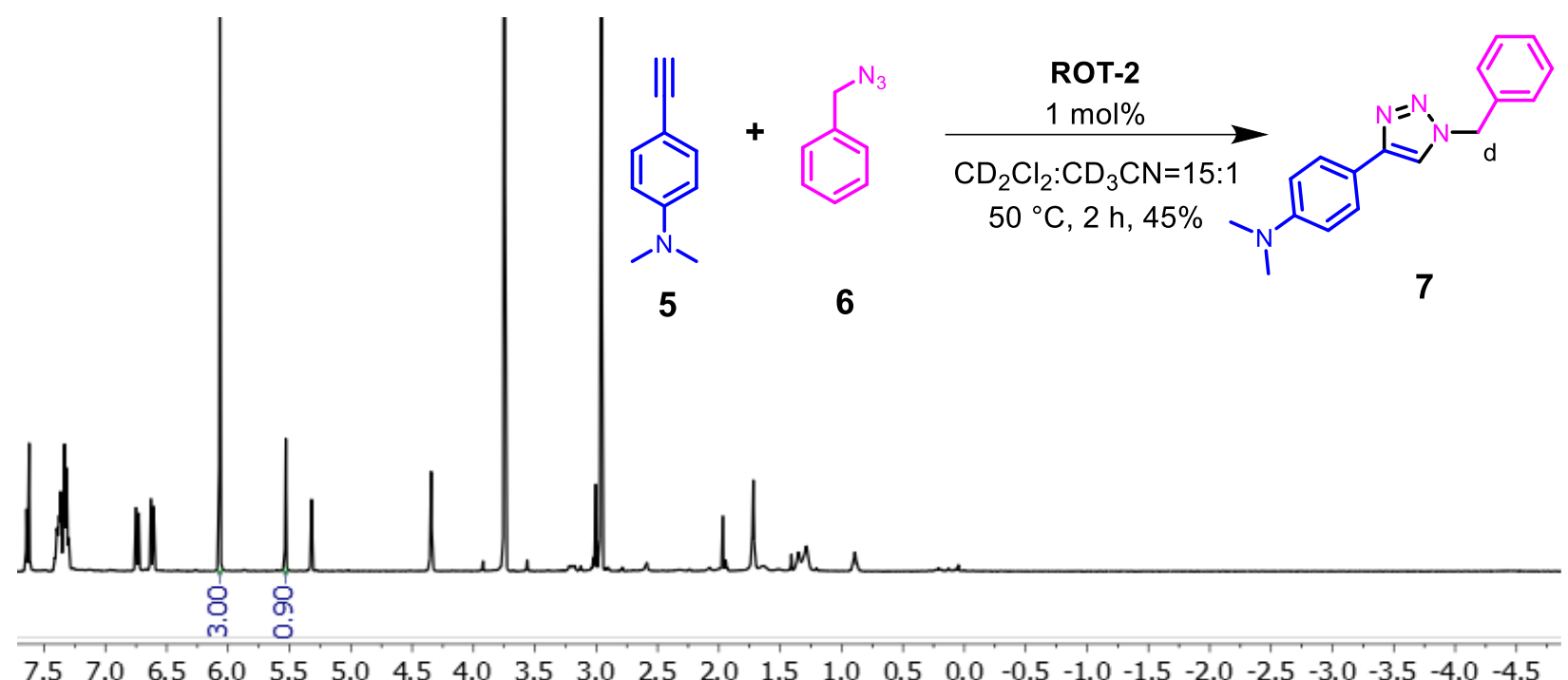

Figure S57: ${ }^{1} \mathrm{H}$ NMR $\left(\mathrm{CD}_{2} \mathrm{Cl}_{2}: \mathrm{CD}_{3} \mathrm{CN}=15: 1,298 \mathrm{~K}, 500 \mathrm{MHz}\right)$ after reacting 5 and $\mathbf{6}$ in presence of $1 \mathrm{~mol} \%$ of ROT-2, showing the formation of $45 \%$ of product 7 . Formation of 7 was quantified via proton $\mathrm{d}-\mathrm{H}(\delta=5.52 \mathrm{ppm})$ in regard to $1,3,5$-trimethoxybenzene $(\delta=6.06 \mathrm{ppm})$.

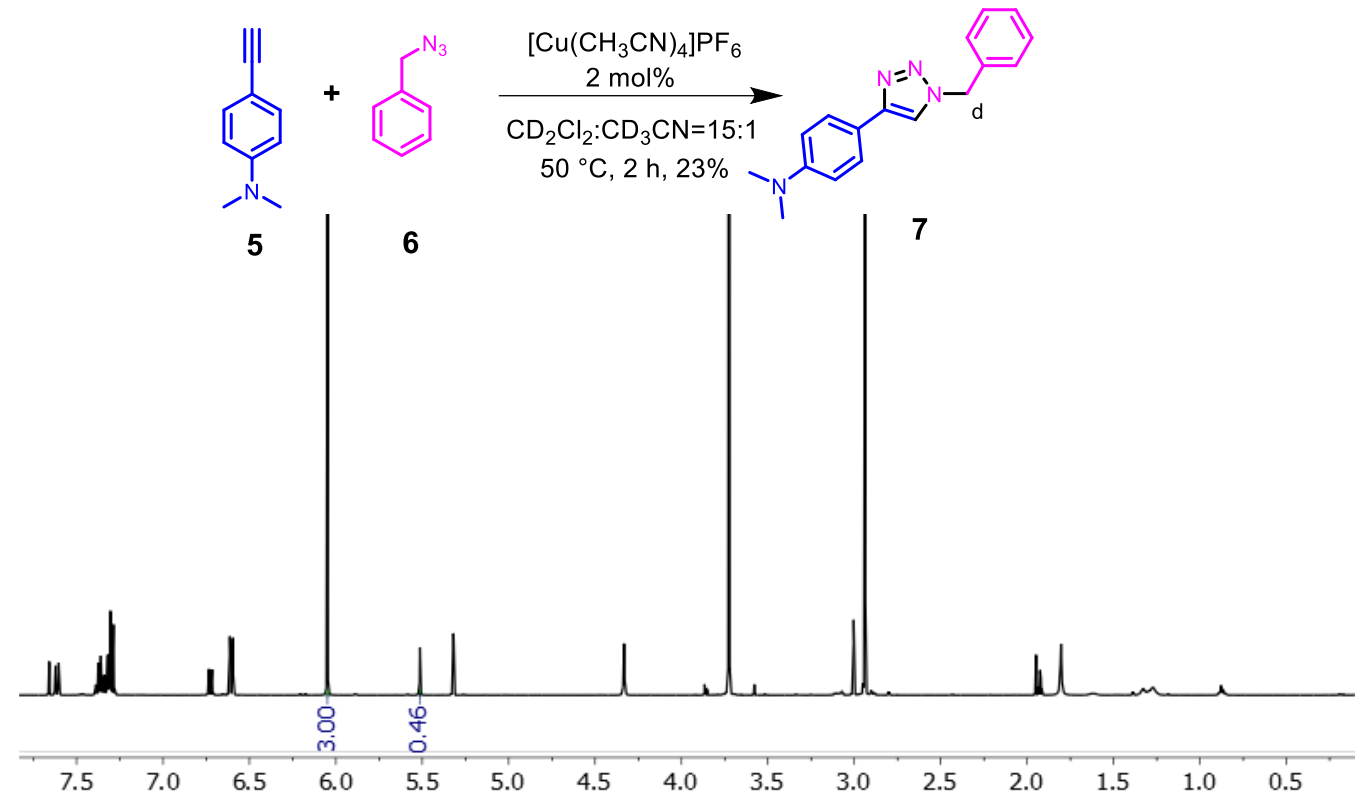

Figure S58: ${ }^{1} \mathrm{H} \mathrm{NMR}\left(\mathrm{CD}_{2} \mathrm{Cl}_{2}: \mathrm{CD}_{3} \mathrm{CN}=15: 1,298 \mathrm{~K}, 500 \mathrm{MHz}\right)$ after reacting 5 and 6 in presence of $2 \mathrm{~mol} \%$ of $\left[\mathrm{Cu}\left(\mathrm{CH}_{3} \mathrm{CN}\right)_{4}\right] \mathrm{PF}_{6}$, showing the formation of $23 \%$ of product 7 that was quantified via proton $\mathrm{d}-\mathrm{H}(\delta=5.52 \mathrm{ppm})$ in regard to $1,3,5$-trimethoxybenzene $(\delta=6.06 \mathrm{ppm})$. 


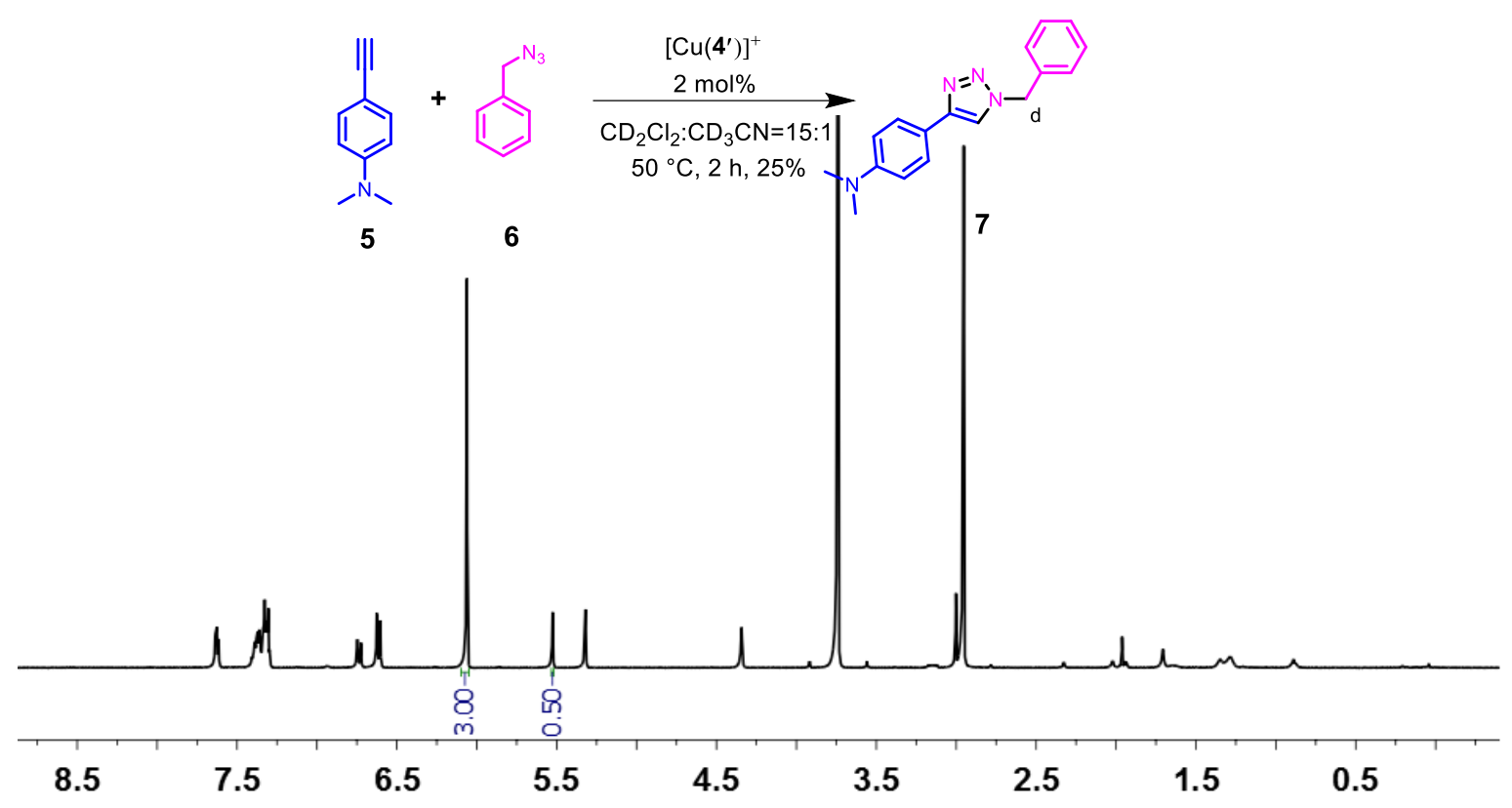

Figure S59: ${ }^{1} \mathrm{H}$ NMR $\left(\mathrm{CD}_{2} \mathrm{Cl}_{2}: \mathrm{CD}_{3} \mathrm{CN}=15: 1,298 \mathrm{~K}, 400 \mathrm{MHz}\right)$ after reacting 5 and $\mathbf{6}$ in presence of $2 \mathrm{~mol} \%$ of $\left[\mathrm{Cu}\left(4^{\prime}\right)\right]^{+}$, showing the formation of $25 \%$ of product 7. Quantification was made via integration of proton $\mathrm{d}-\mathrm{H}(\delta=5.52 \mathrm{ppm})$ in regard to $1,3,5$-trimethoxybenzene $(\delta=6.06 \mathrm{ppm})$.

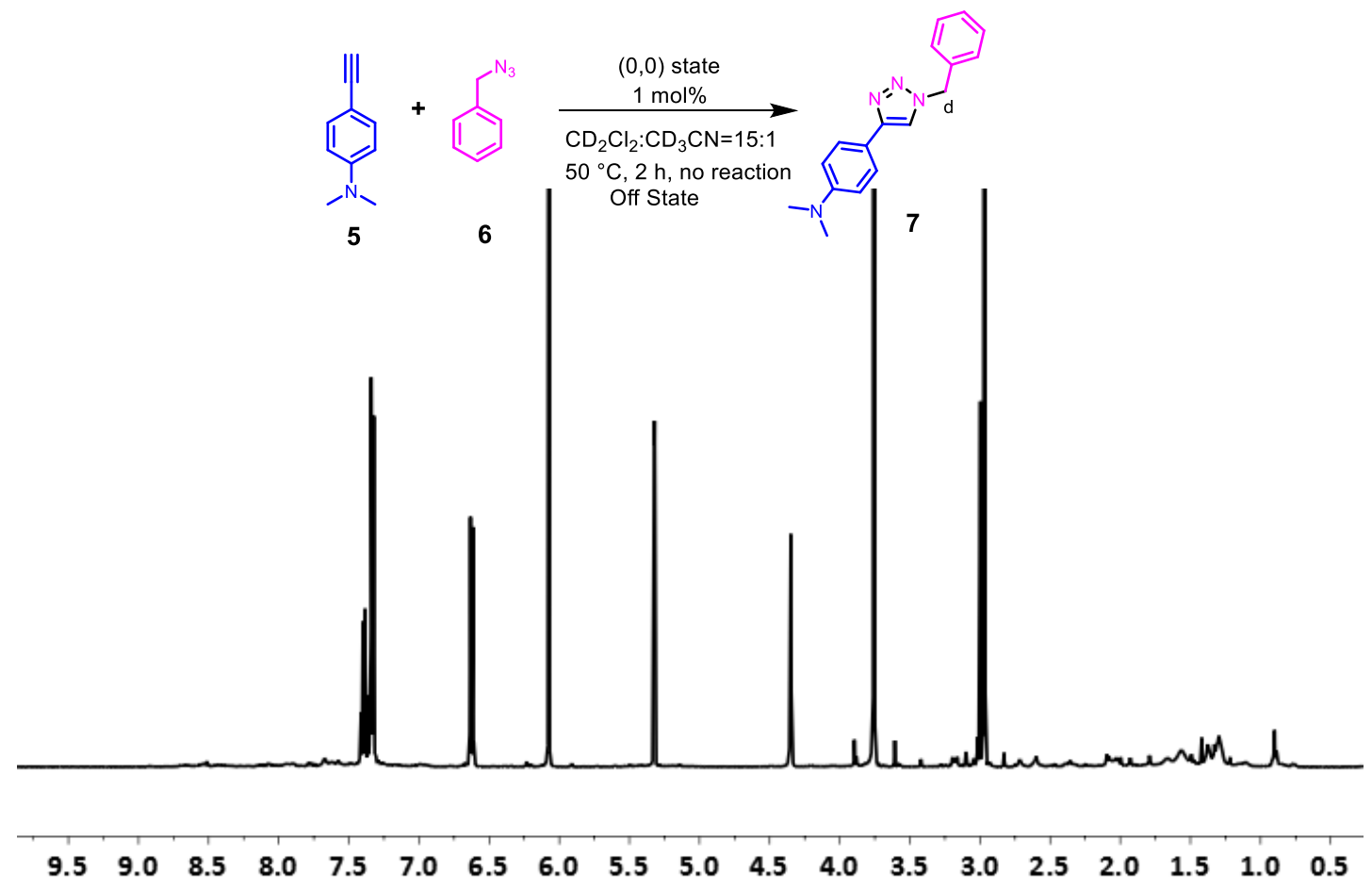

Figure S60: ${ }^{1} \mathrm{H}$ NMR $\left(\mathrm{CD}_{2} \mathrm{Cl}_{2}: \mathrm{CD}_{3} \mathrm{CN}=15: 1,298 \mathrm{~K}, 500 \mathrm{MHz}\right)$ after reacting 5 and $\mathbf{6}$ in presence of $1 \mathrm{~mol} \%$ of the 12 -component logic gate in state $(0,0)$, showing no formation of product 7 (no singlet at $\delta=5.52$ ). 


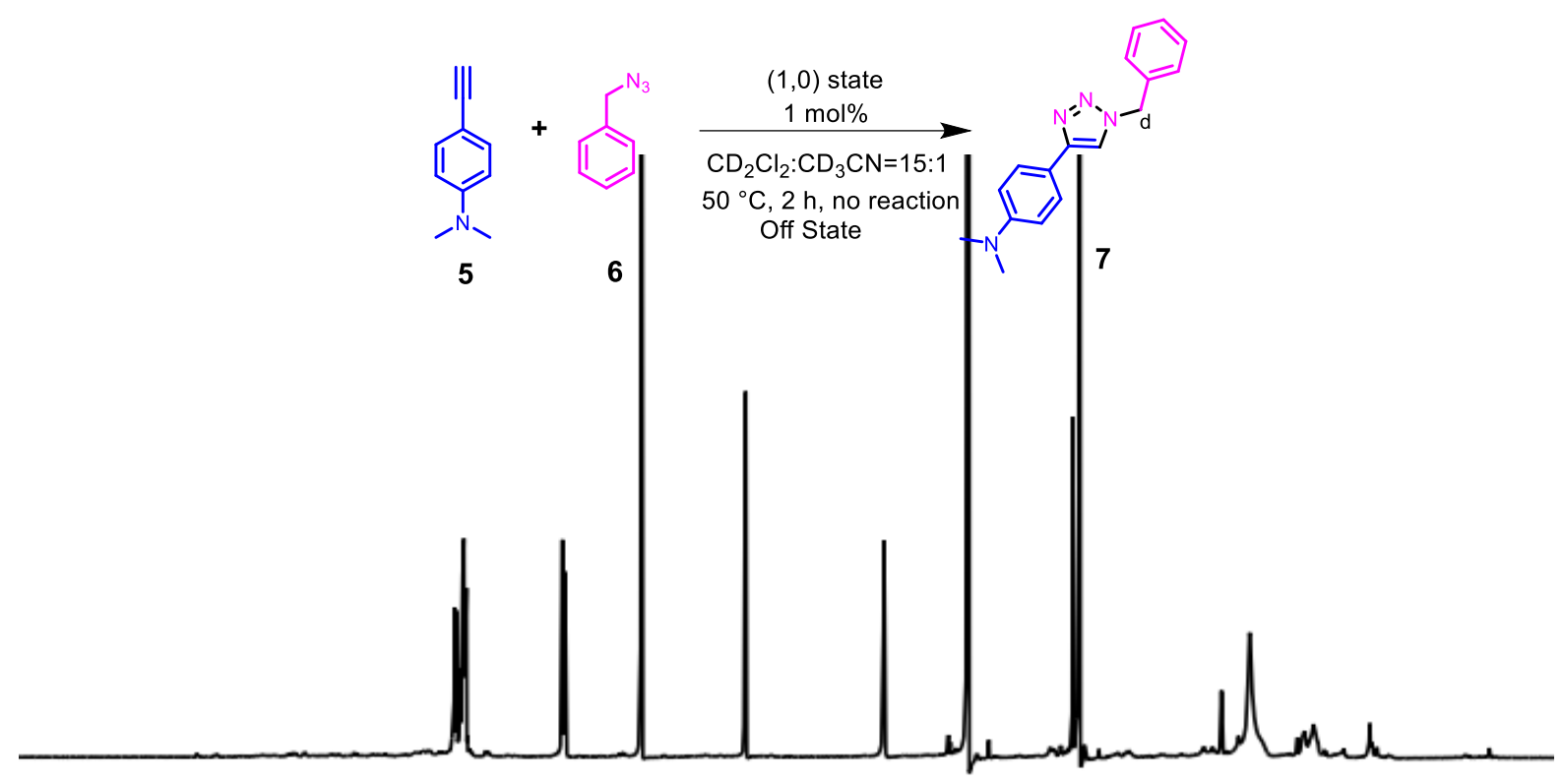

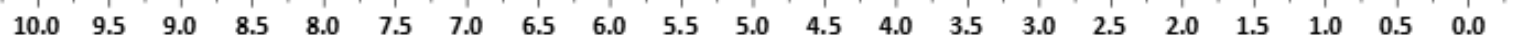

Figure S61: ${ }^{1} \mathrm{H}$ NMR $\left(\mathrm{CD}_{2} \mathrm{Cl}_{2}: \mathrm{CD}_{3} \mathrm{CN}=15: 1,298 \mathrm{~K}, 500 \mathrm{MHz}\right)$ after reacting 5 and 6 in presence of $1 \mathrm{~mol} \%$ of the 12-component logic gate in state $(1,0)$, showing no formation of product 7 (no singlet at $\delta=5.52$ ).

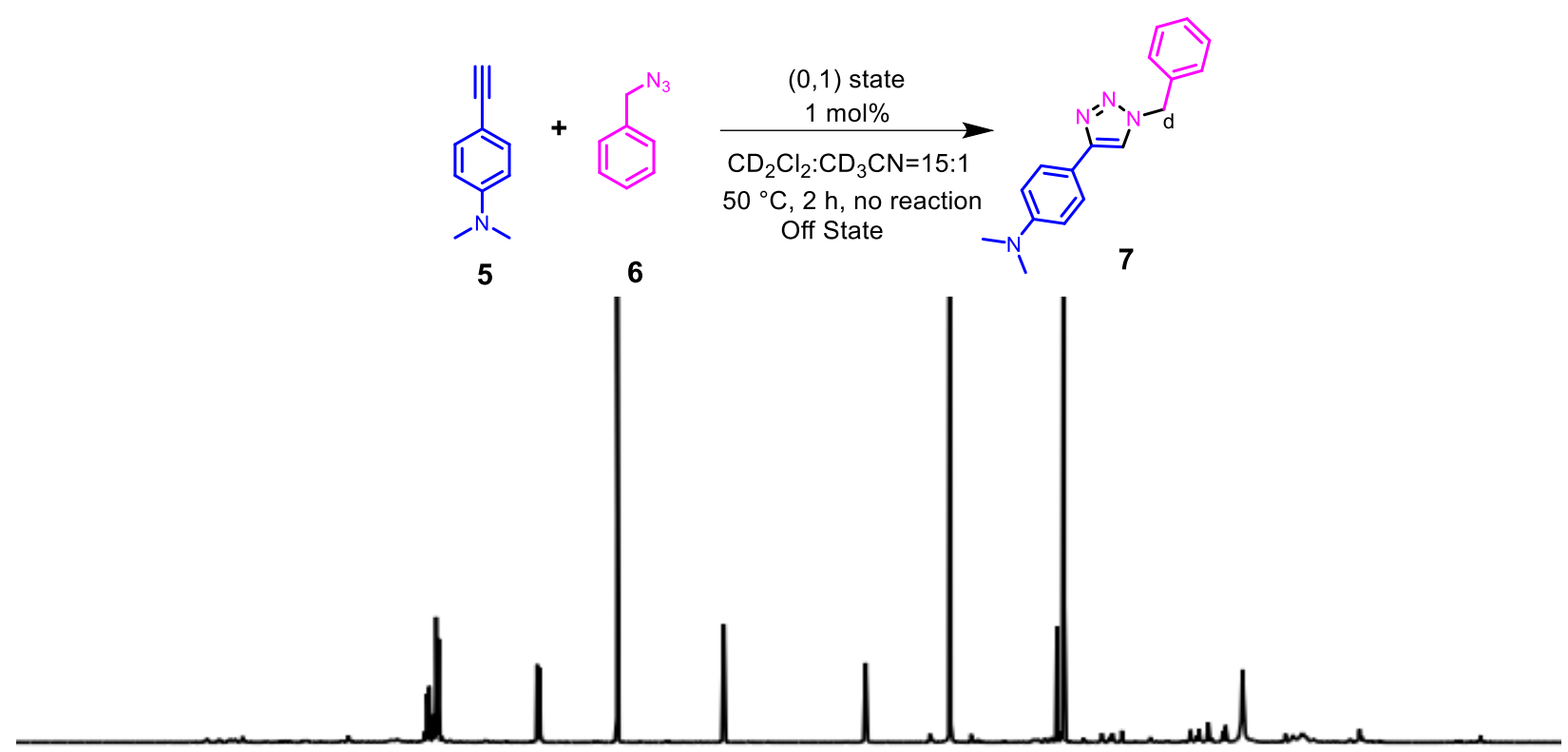

$\begin{array}{llllllllllllllllllllll}10.0 & 9.5 & 9.0 & 8.5 & 8.0 & 7.5 & 7.0 & 6.5 & 6.0 & 5.5 & 5.0 & 4.5 & 4.0 & 3.5 & 3.0 & 2.5 & 2.0 & 1.5 & 1.0 & 0.5 & 0.0 & -0\end{array}$

Figure S62: ${ }^{1} \mathrm{H}$ NMR $\left(\mathrm{CD}_{2} \mathrm{Cl}_{2}: \mathrm{CD}_{3} \mathrm{CN}=15: 1,298 \mathrm{~K}, 500 \mathrm{MHz}\right)$ after reacting 5 and 6 in presence of $1 \mathrm{~mol} \%$ of the 12-component logic gate in state $(0,1)$, showing no formation of product 7 (no singlet at $\delta=5.52$ ). 


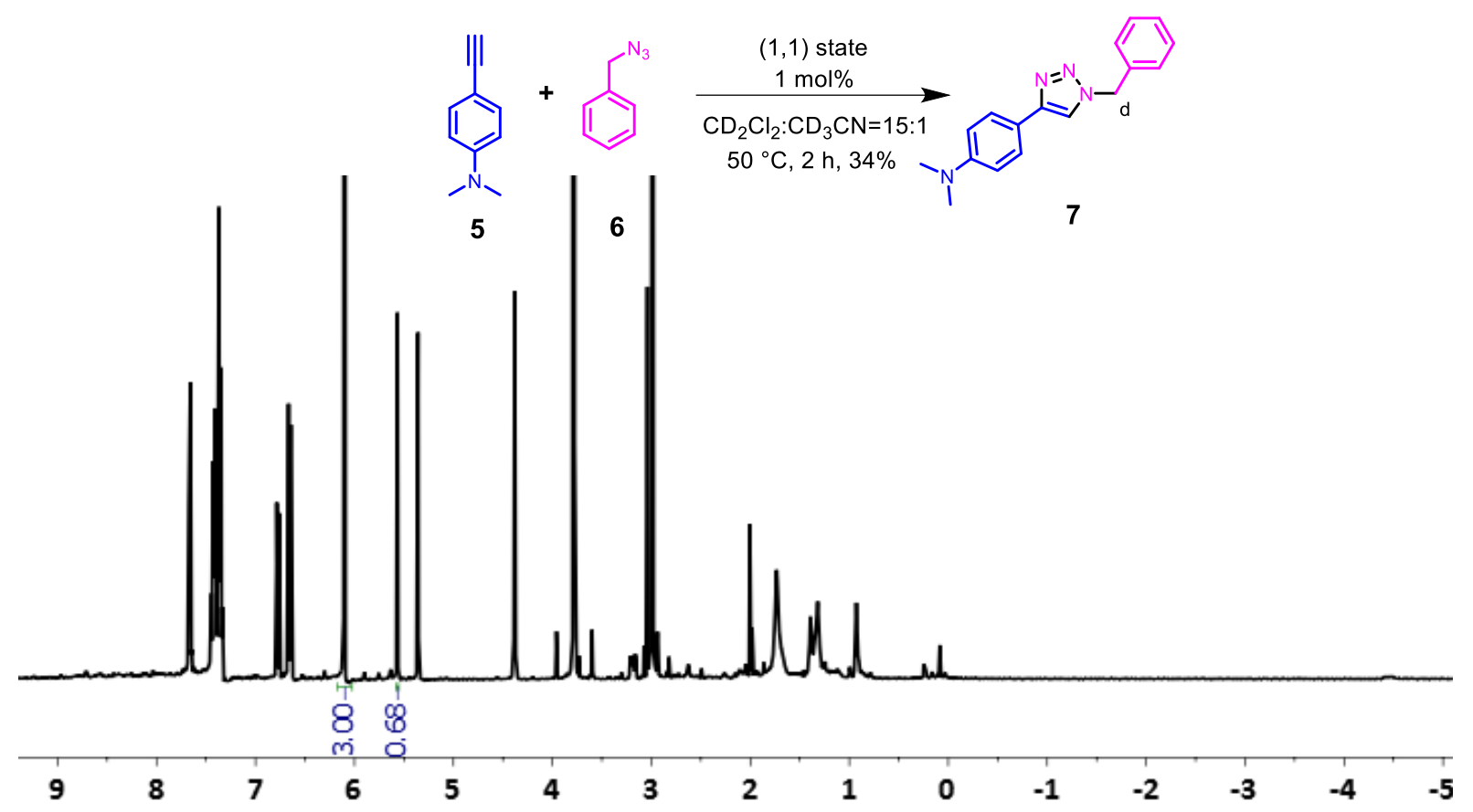

Figure S63: ${ }^{1} \mathrm{H}$ NMR $\left(\mathrm{CD}_{2} \mathrm{Cl}_{2}: \mathrm{CD}_{3} \mathrm{CN}=15: 1,298 \mathrm{~K}, 400 \mathrm{MHz}\right)$ after reacting 5 and 6 in presence of $1 \mathrm{~mol} \%$ of the 12 -component logic gate in state $(1,1)$ state, showing formation of product 7 in $34 \%$ yield that was quantified via proton $\mathrm{d}-\mathrm{H}(\delta=5.52 \mathrm{ppm})$ in regard to $1,3,5$-trimethoxybenzene $(\delta=6.06 \mathrm{ppm})$. 


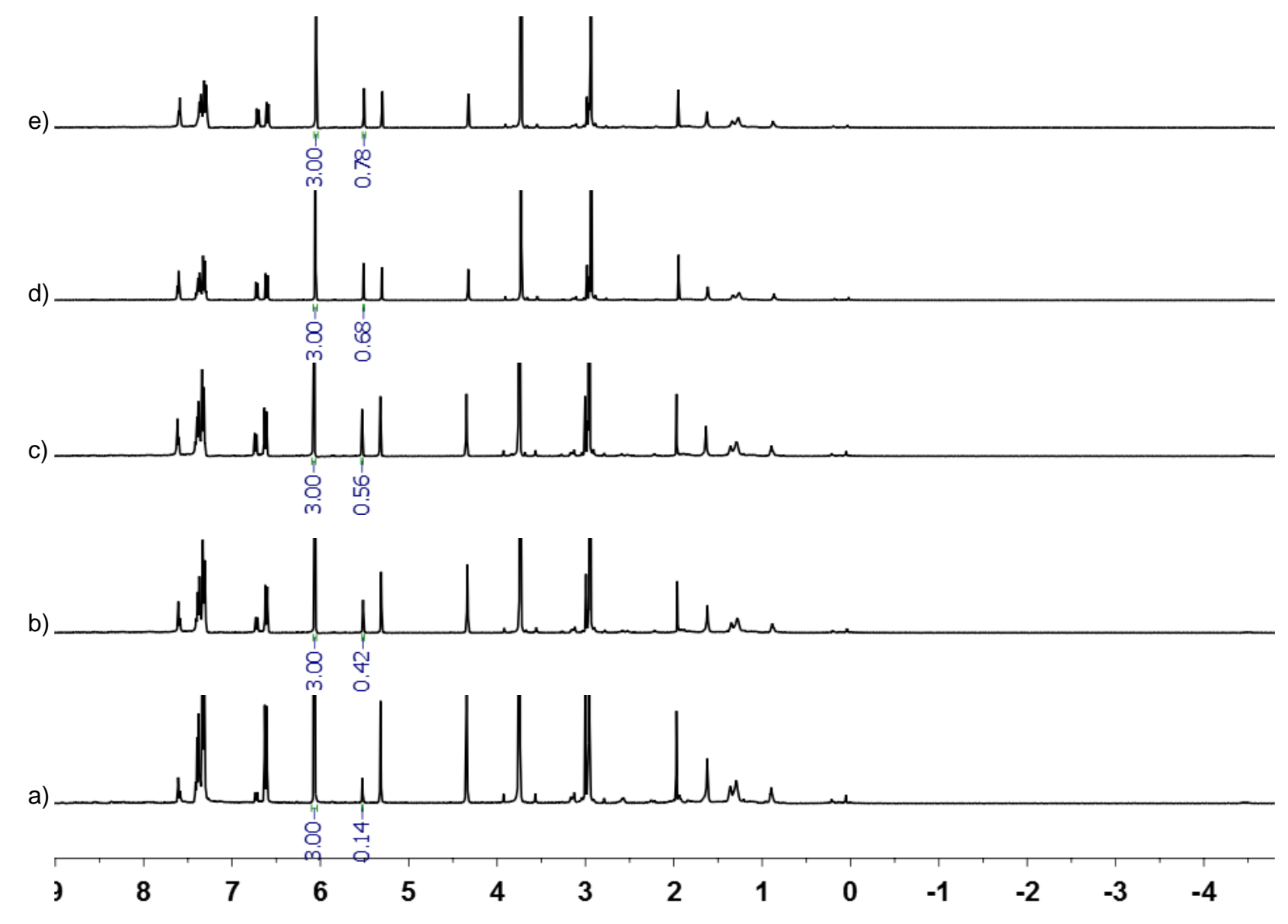

Figure S64: ${ }^{1} \mathrm{H}$ NMR $\left(\mathrm{CD}_{2} \mathrm{Cl}_{2}: \mathrm{CD}_{3} \mathrm{CN}=15: 1,400 \mathrm{MHz}\right)$ spectra obtained during heating 5 and 6 at $50{ }^{\circ} \mathrm{C}$ in presence of $1 \mathrm{~mol} \%$ of AND gate after activating state $(1,1)$. Samples were taken after a) $30 \mathrm{~min}$ (yield 7\%), b) $60 \mathrm{~min}$ (yield 21\%), c) $90 \mathrm{~min}$ (yield 28\%), d) $120 \mathrm{~min}$ (yield $34 \%$ ), and e) $150 \mathrm{~min}$ (yield 39\%). Due to the delayed release of $\mathrm{Cu}^{+}$from the ensemble gate, initial conversion is slower than expected. 

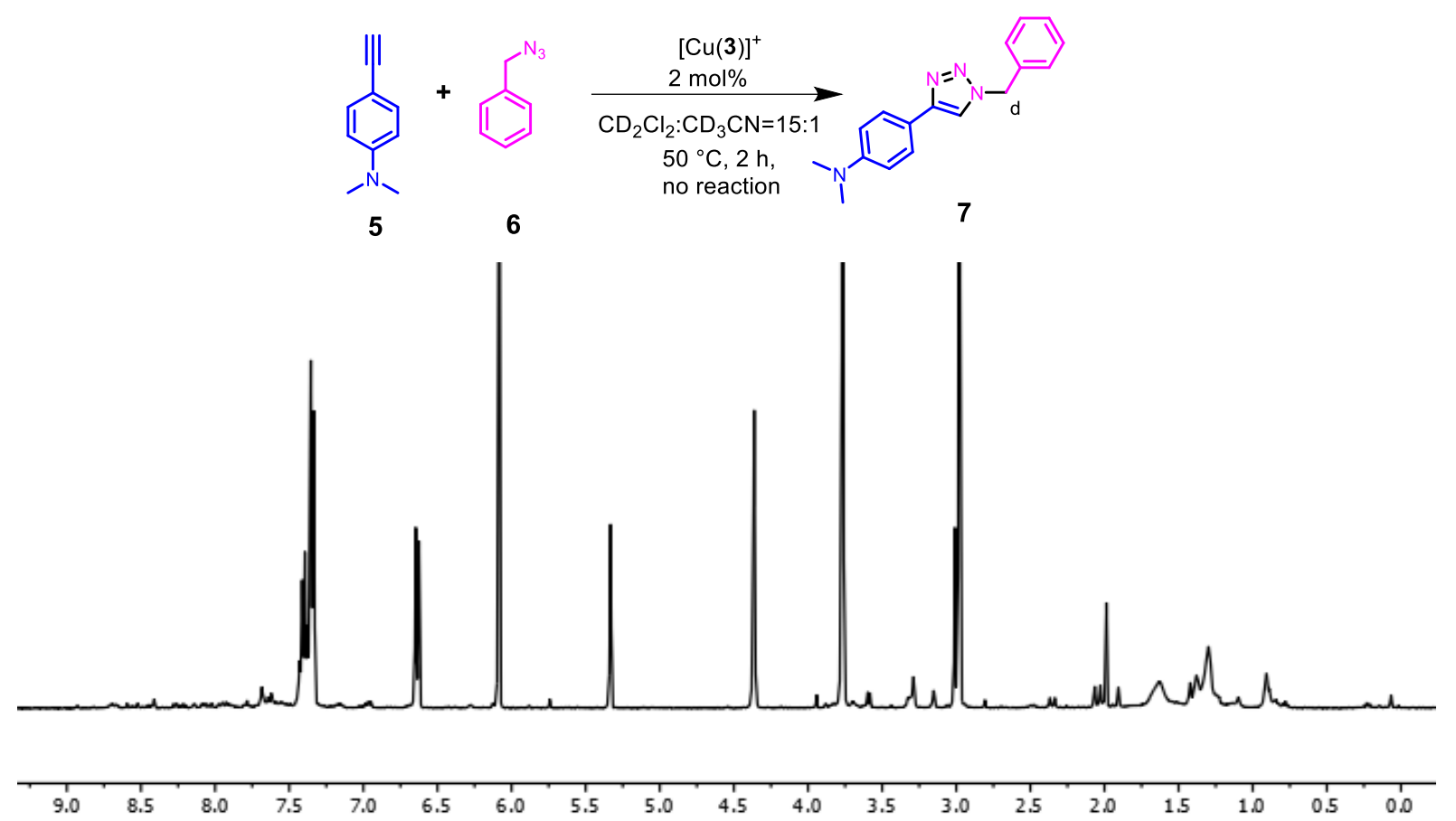

Figure S65: ${ }^{1} \mathrm{H}$ NMR $\left(\mathrm{CD}_{2} \mathrm{Cl}_{2}: \mathrm{CD}_{3} \mathrm{CN}=15: 1,298 \mathrm{~K}, 500 \mathrm{MHz}\right)$ after reacting 5 and $\mathbf{6}$ in presence of $2 \mathrm{~mol} \%$ of $[\mathrm{Cu}(3)]^{+}$, showing no product (7) formation (no singlet at $\delta=5.52$ ).

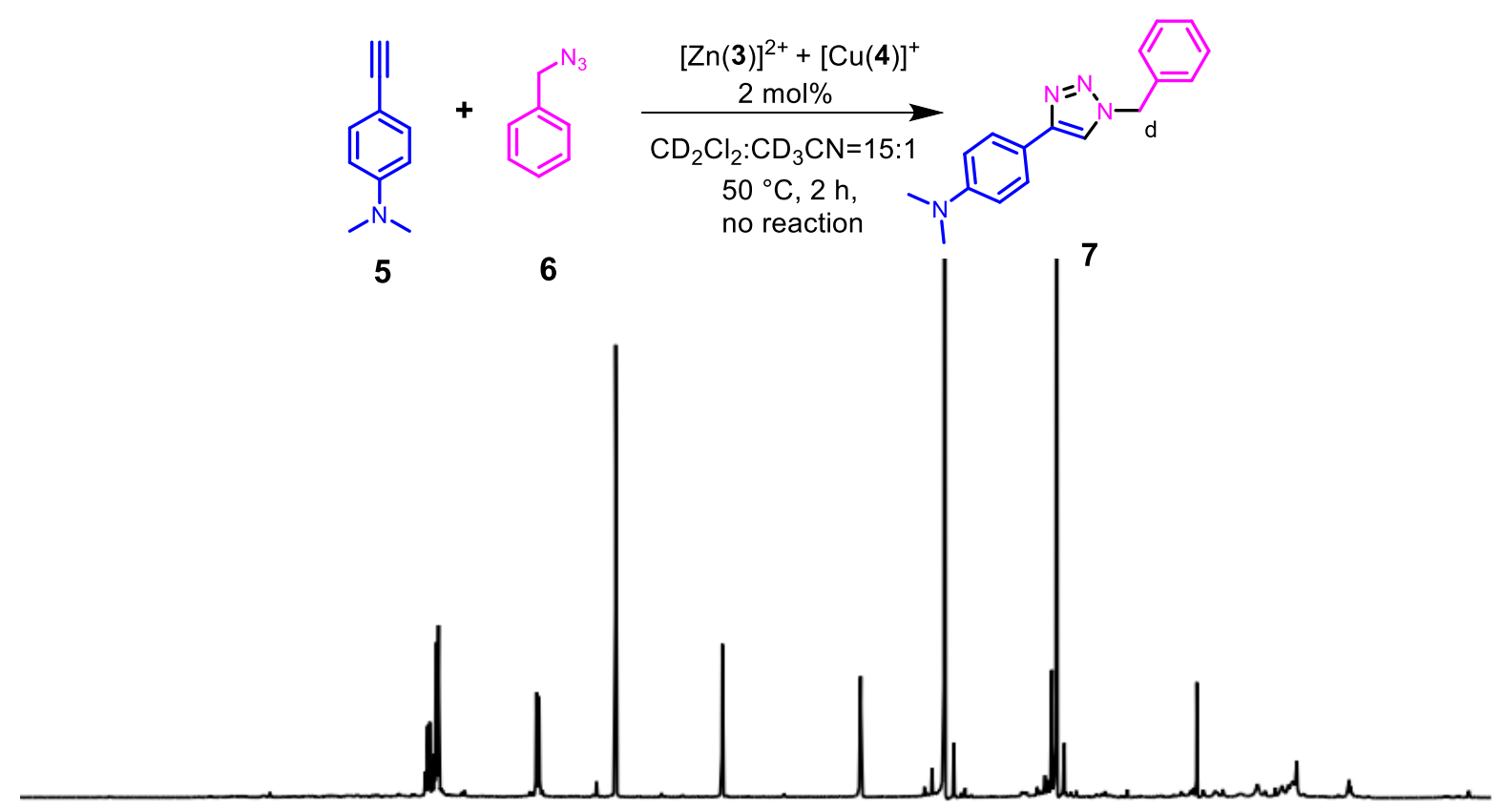

$\begin{array}{lllllllllllllllllllll}10.0 & 9.5 & 9.0 & 8.5 & 8.0 & 7.5 & 7.0 & 6.5 & 6.0 & 5.5 & 5.0 & 4.5 & 4.0 & 3.5 & 3.0 & 2.5 & 2.0 & 1.5 & 1.0 & 0.5 & 0.0\end{array}$

Figure S66: ${ }^{1} \mathrm{H}$ NMR $\left(\mathrm{CD}_{2} \mathrm{Cl}_{2}: \mathrm{CD}_{3} \mathrm{CN}=15: 1, \mathrm{~K}, 500 \mathrm{MHz}\right)$ after reacting 5 and $\mathbf{6}$ in presence of $2 \mathrm{~mol} \%$ of $[\mathrm{Zn}(3)]^{2+}+[\mathrm{Cu}(\mathbf{4})]^{+}$, showing no product (7) formation (no singlet at $\delta=5.52$ ). 

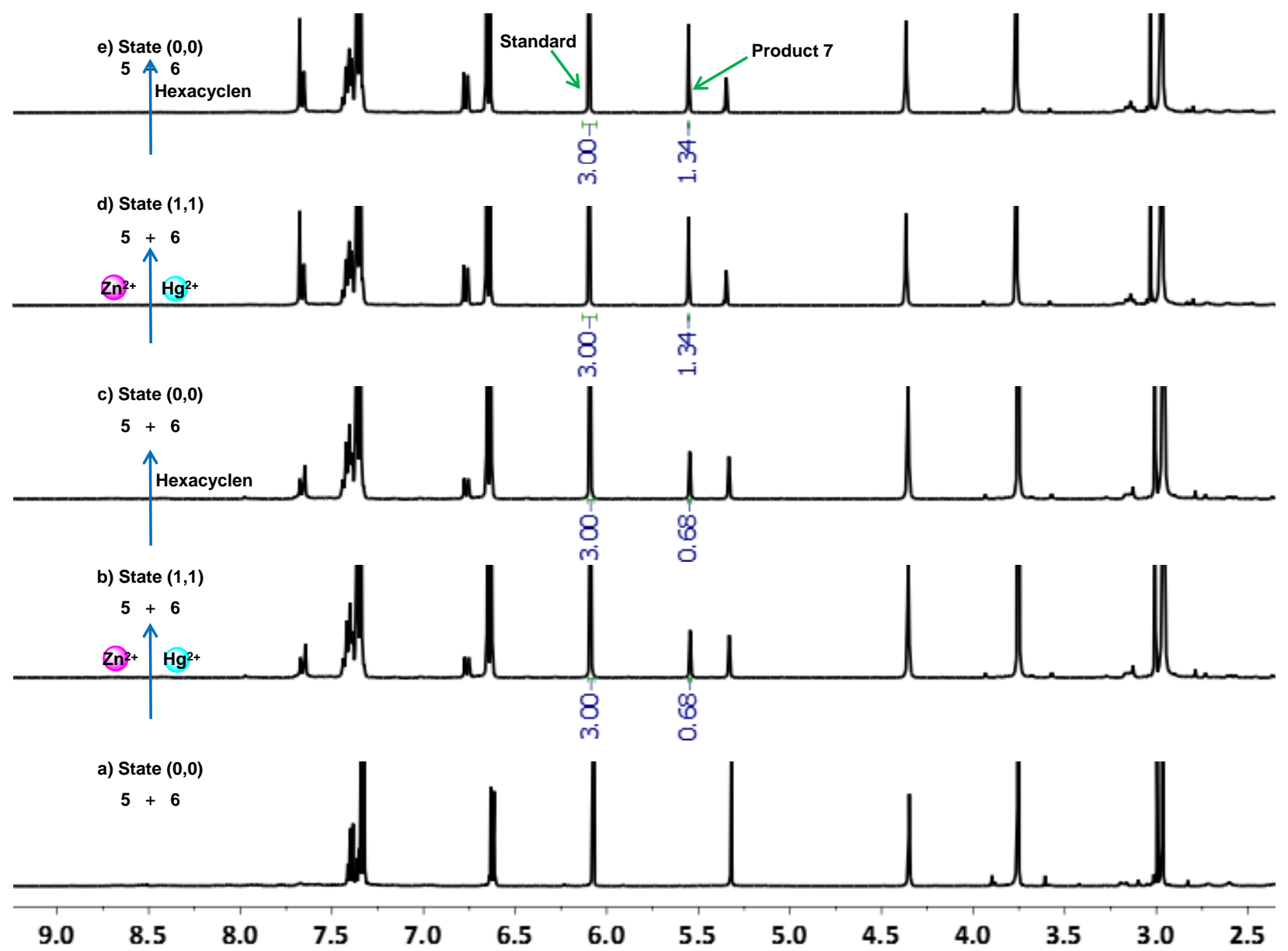

Figure S67: Partial ${ }^{1} \mathrm{H}$ NMR (400 MHz, $\mathrm{CD}_{2} \mathrm{Cl}_{2}: \mathrm{CD}_{3} \mathrm{CN}=15: 1$ ) spectrum obtained after (a) heating the reaction mixture of 5 and 6 in presence of $1 \mathrm{~mol} \%(0,0)$ state of AND gate at $50{ }^{\circ} \mathrm{C}$ for $2 \mathrm{~h}$ in an NMR tube revealed that click catalysis was OFF (no singlet at $\delta=5.52$ ). (b) After addition of 2.0 equiv. of $\mathrm{Zn}(\mathrm{OTf})_{2}$ and 2.0 equiv. of $\mathrm{Hg}\left(\mathrm{ClO}_{4}\right)_{2}$ with respect to stator 1 and subsequent heating at $50{ }^{\circ} \mathrm{C}$ for $2 \mathrm{~h}$ click product 7 was formed (yield $=34 \%$ calculated with respect to the internal standard). (c) After adding the consumed substrates and 4.0 equiv. of hexacyclen with respect to 1 and heating at $50{ }^{\circ} \mathrm{C}$ for $2 \mathrm{~h}$. (d) Addition of 2.0 equiv. of $\mathrm{Zn}(\mathrm{OTf})_{2}$ and 2.0 equiv. of $\mathrm{Hg}\left(\mathrm{ClO}_{4}\right)_{2}$ with respect to 1 and heating at $50{ }^{\circ} \mathrm{C}$ for $2 \mathrm{~h}$ resulted in an increase of the click product 7 by $33 \%$ (total yield $=67 \%$ ). (e) After adding the consumed substrates and 4.0 equiv. of hexacyclen with respect to 1 and heating at $50{ }^{\circ} \mathrm{C}$ for $2 \mathrm{~h}$. 


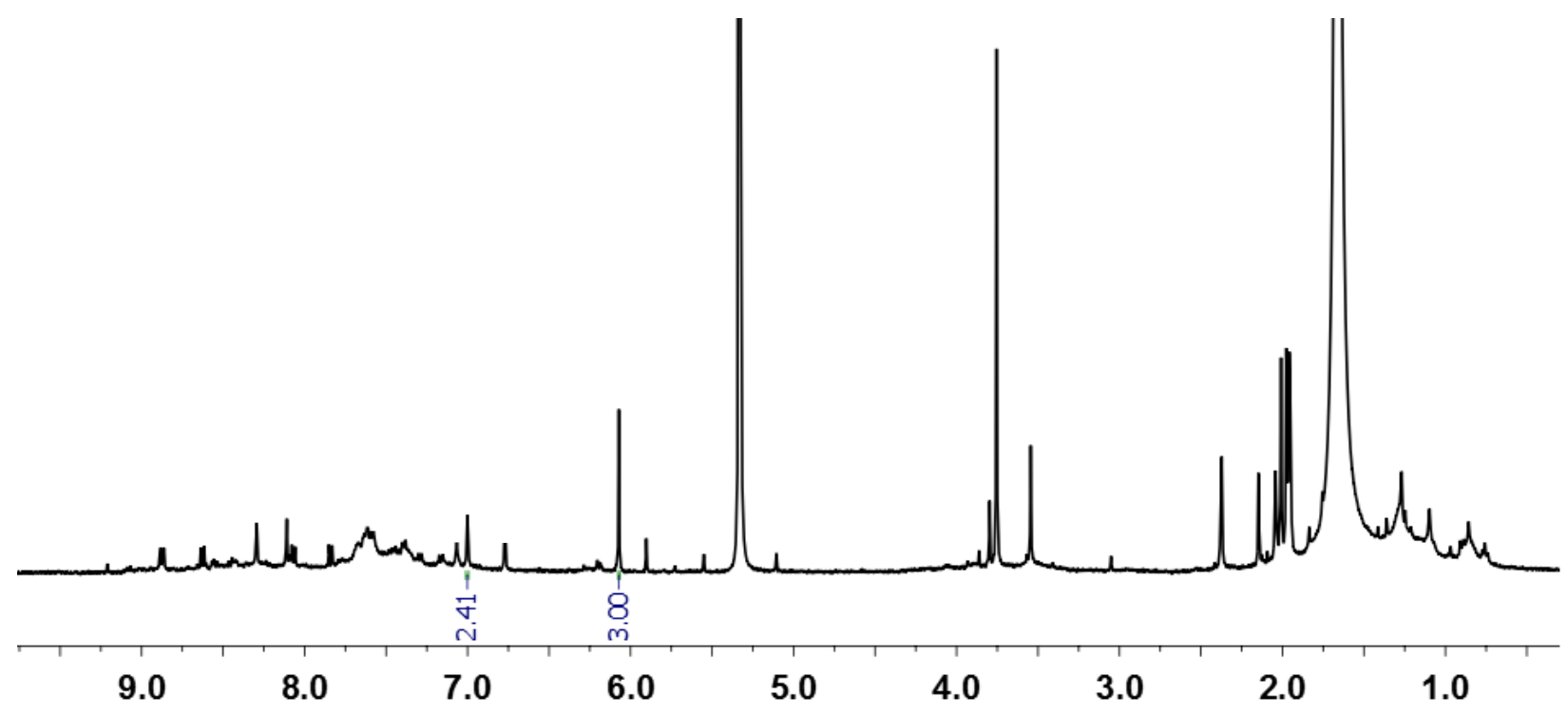

Figure S68: ${ }^{1} \mathrm{H}$ NMR spectra of quantification of copper(I) release, ascertained by using 2,9dimesitylphenanthroline (4') as the copper(I) binding receptor. 1,3,5-Trimethoxybenzene $(\delta=6.06$ ppm) was used in a 1:1 ratio with respect to $\mathbf{4}^{\prime}$ as internal standard. Signal corresponding to 9-H of $\left[\mathrm{Cu}\left(\mathbf{4}^{\prime}\right)\right]^{+}$(at $6.99 \mathrm{ppm}$ ) is used to measure the release of copper(I) into solution. It shows that $60 \%(2.41 / 4 \times 100=60 \%)$ of copper(I) is released which is captured by $4^{\prime}$. 


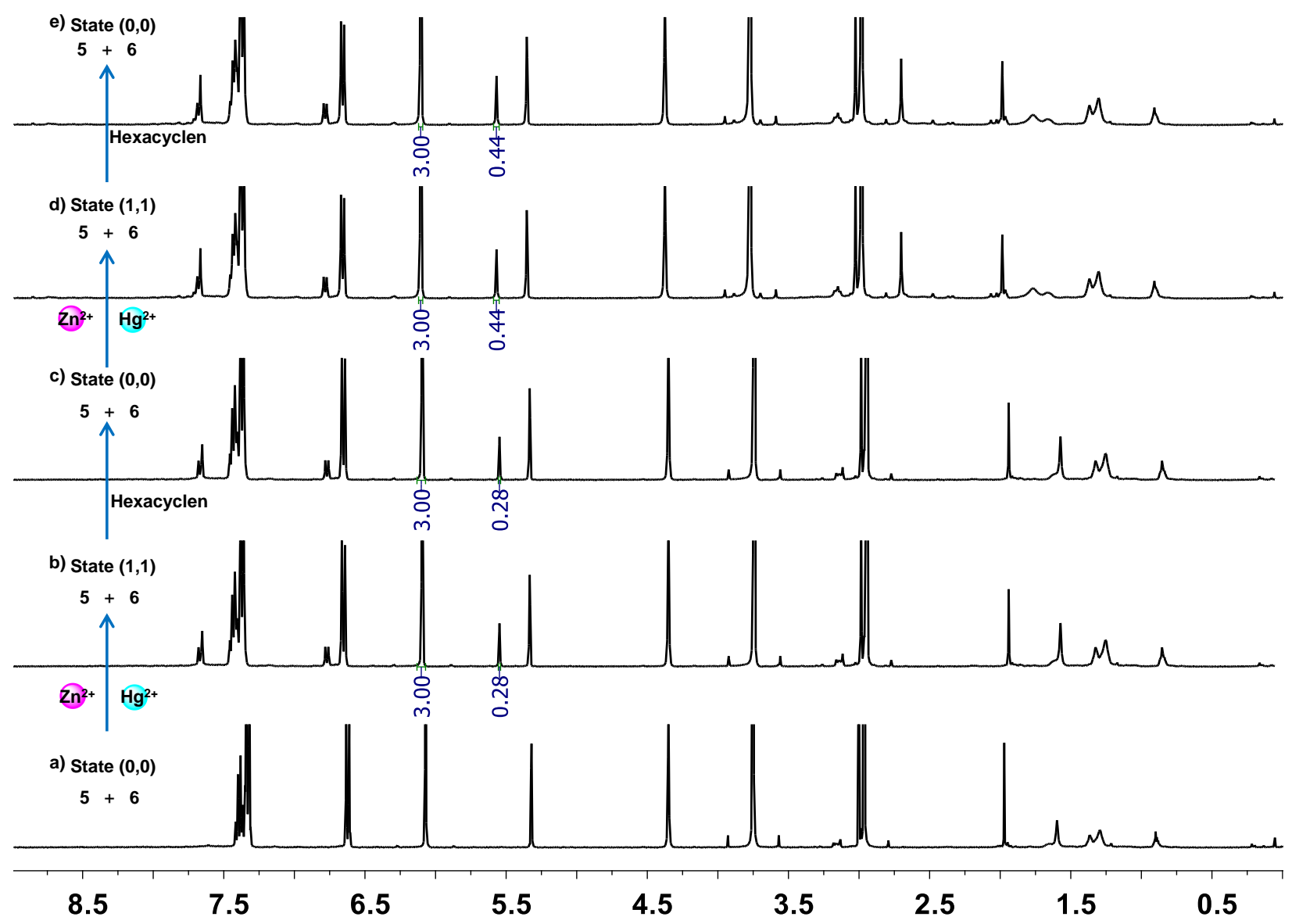

Figure S69: ${ }^{1} \mathrm{H}$ NMR (400 MHz, $\mathrm{CD}_{2} \mathrm{Cl}_{2}: \mathrm{CD}_{3} \mathrm{CN}=15: 1$ ) spectrum obtained after (a) heating the reaction mixture of 5 and 6 in presence of $2 \mathrm{~mol} \%$ of both $\left([\mathrm{Cu}(3)]^{+}+4\right)$ at $50{ }^{\circ} \mathrm{C}$ for $2 \mathrm{~h}$ in an NMR tube revealing that click catalysis was OFF (no singlet at $\delta=5.52$ ). (b) After addition of 2 mol\% of $\mathrm{Zn}(\mathrm{OTf})_{2}$ and $2 \mathrm{~mol} \%$ of $\mathrm{Hg}\left(\mathrm{ClO}_{4}\right)_{2}$ with respect to compound 5 and subsequent heating at $50{ }^{\circ} \mathrm{C}$ for $2 \mathrm{~h}$, click product 7 was formed in $14 \%$ yield (calculated with respect to the internal standard). (c) After adding the consumed substrates and $4 \mathrm{~mol} \%$ of hexacyclen (with respect to 5 ) further heating at $50{ }^{\circ} \mathrm{C}$ for $2 \mathrm{~h}$ did not provide new click product. (d) Addition of $2 \mathrm{~mol} \%$ of $\mathrm{Zn}(\mathrm{OTf})_{2}$ and $2 \mathrm{~mol} \%$ of $\mathrm{Hg}\left(\mathrm{ClO}_{4}\right)_{2}$ with respect to 5 and heating at $50{ }^{\circ} \mathrm{C}$ for $2 \mathrm{~h}$ resulted in an increase of click product 7 by $8 \%$ (total yield $=22 \%$ ). (e) After adding the consumed substrates and $4 \mathrm{~mol} \%$ of hexacyclen (with respect to 5 ) further heating at $50{ }^{\circ} \mathrm{C}$ for $2 \mathrm{~h}$ did not provide new click product. 


\section{ESI-MS Spectra}

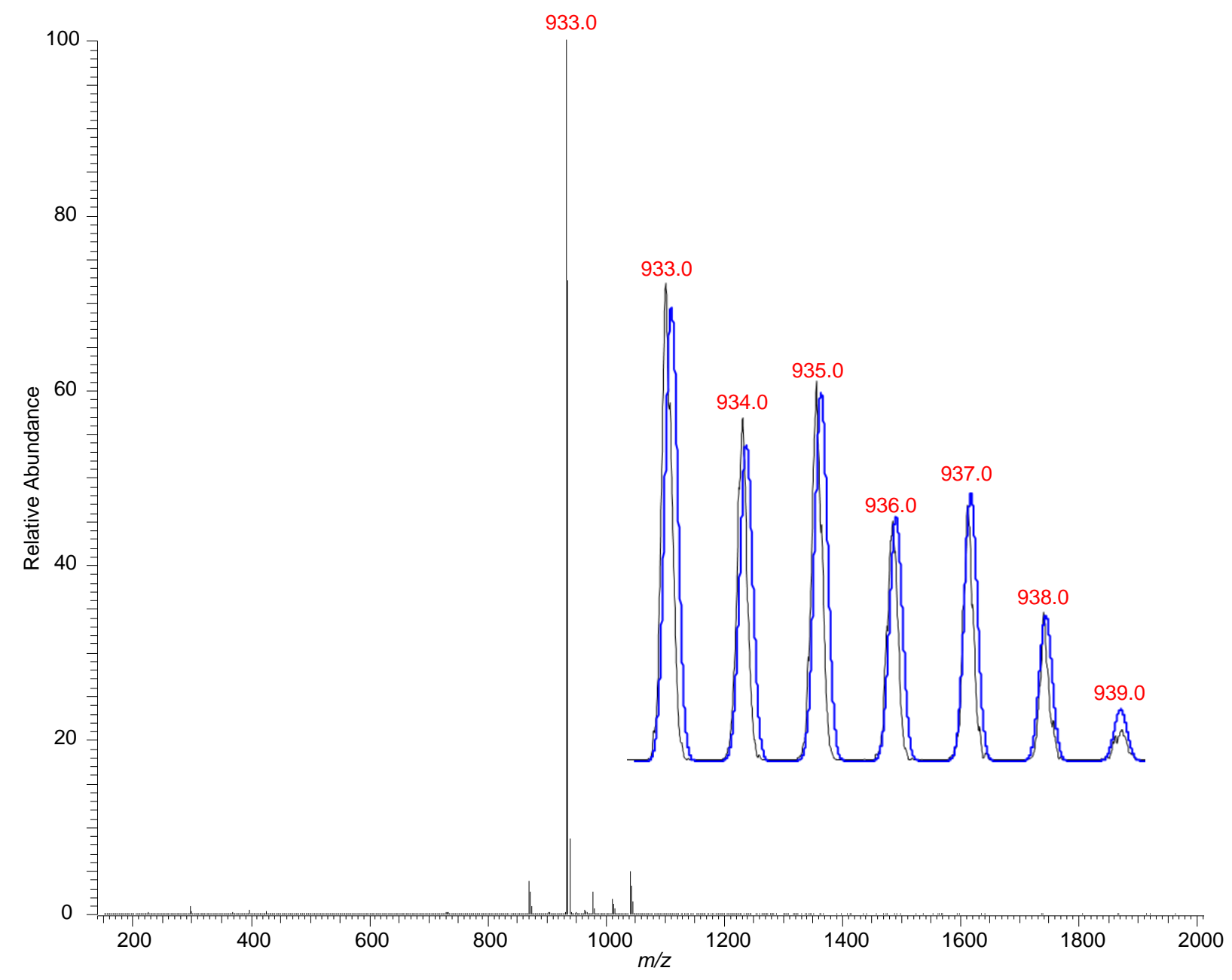

Figure S70: ESI-MS spectrum of rotator $\left[2+\mathrm{H}^{+}\right]$. 


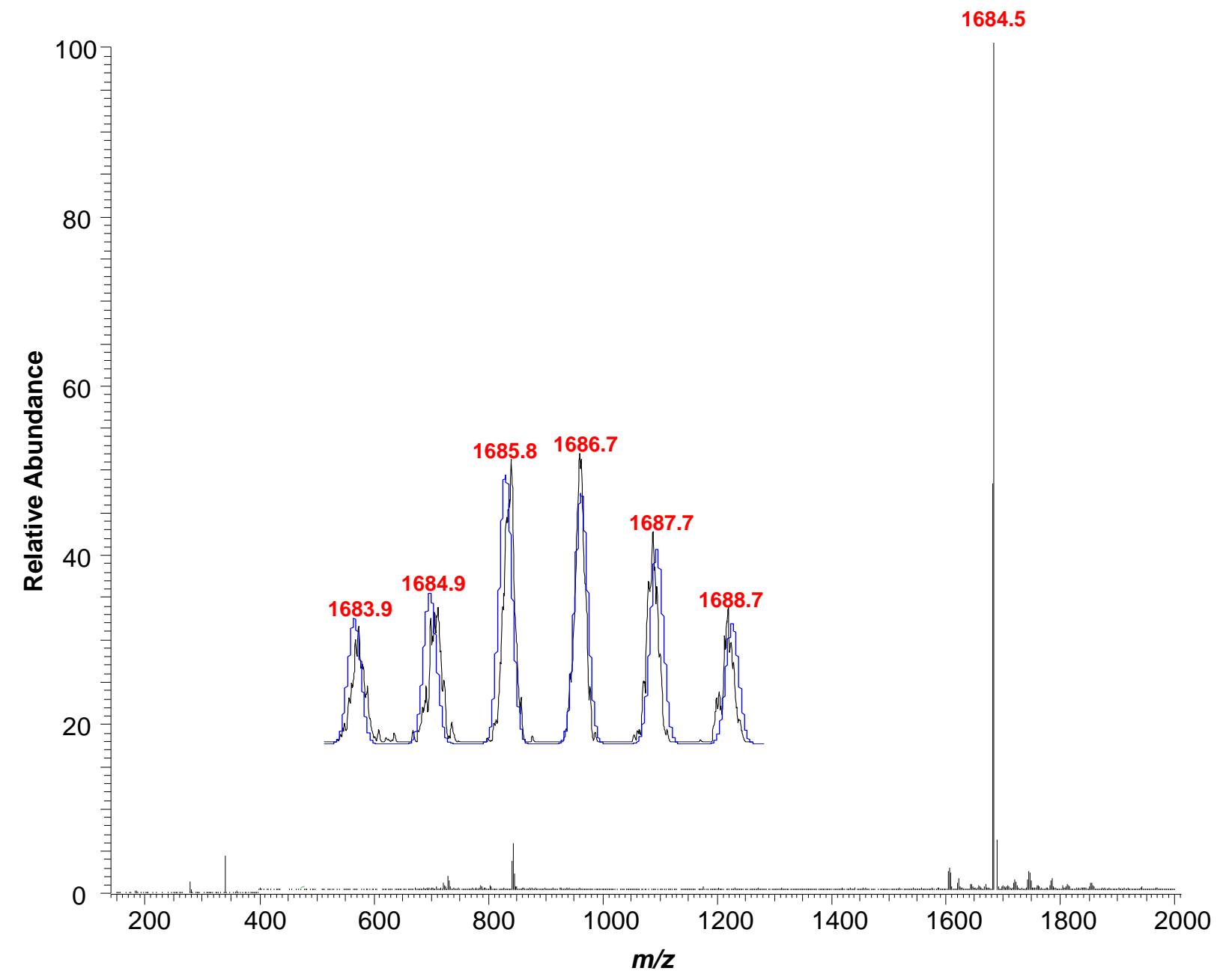

Figure S71: ESI-MS spectrum of complex $[\mathrm{Cu}(3)]^{+}$. 


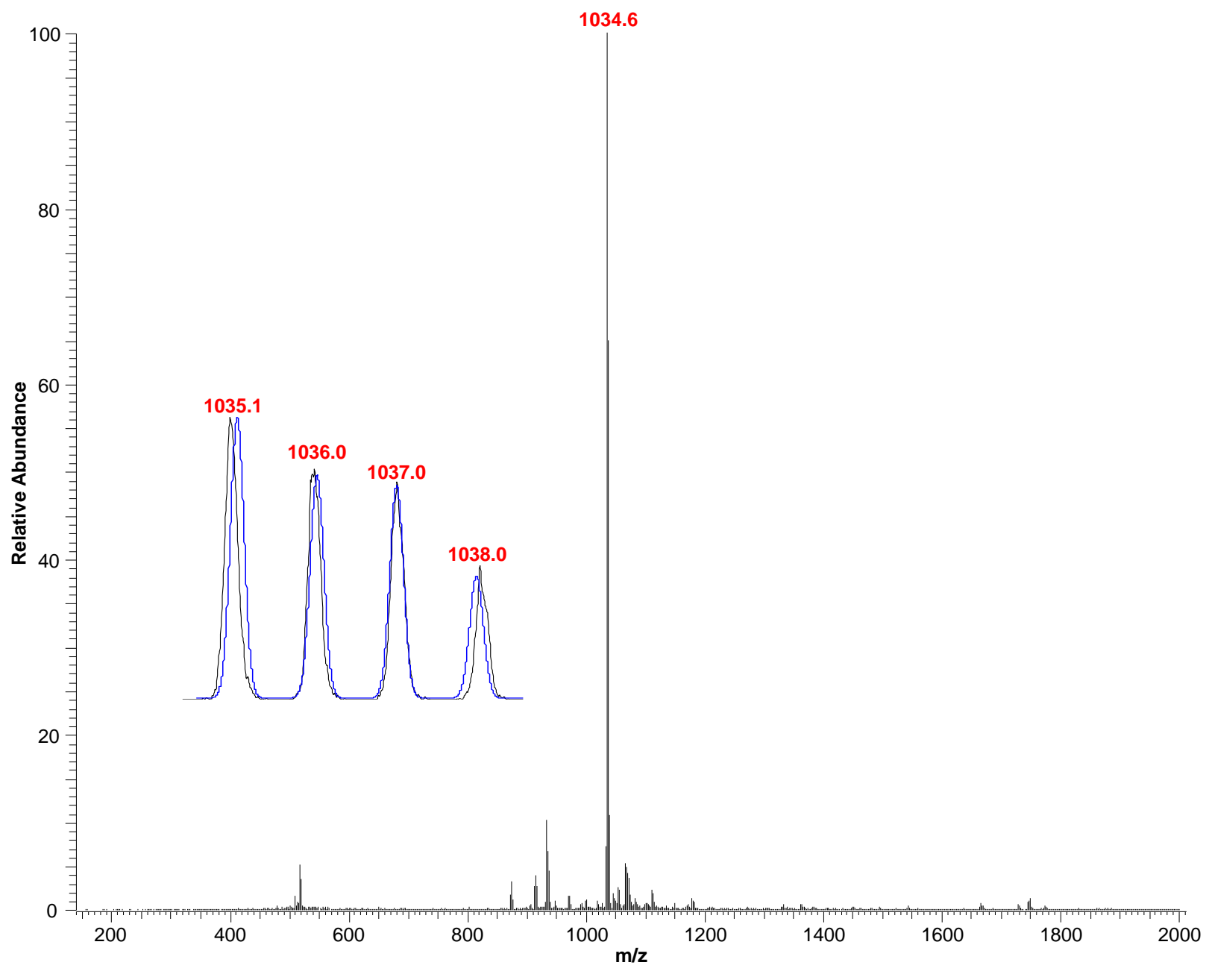

Figure S72: ESI-MS spectrum of complex $[\mathrm{Cu}(4)]^{+}$. 


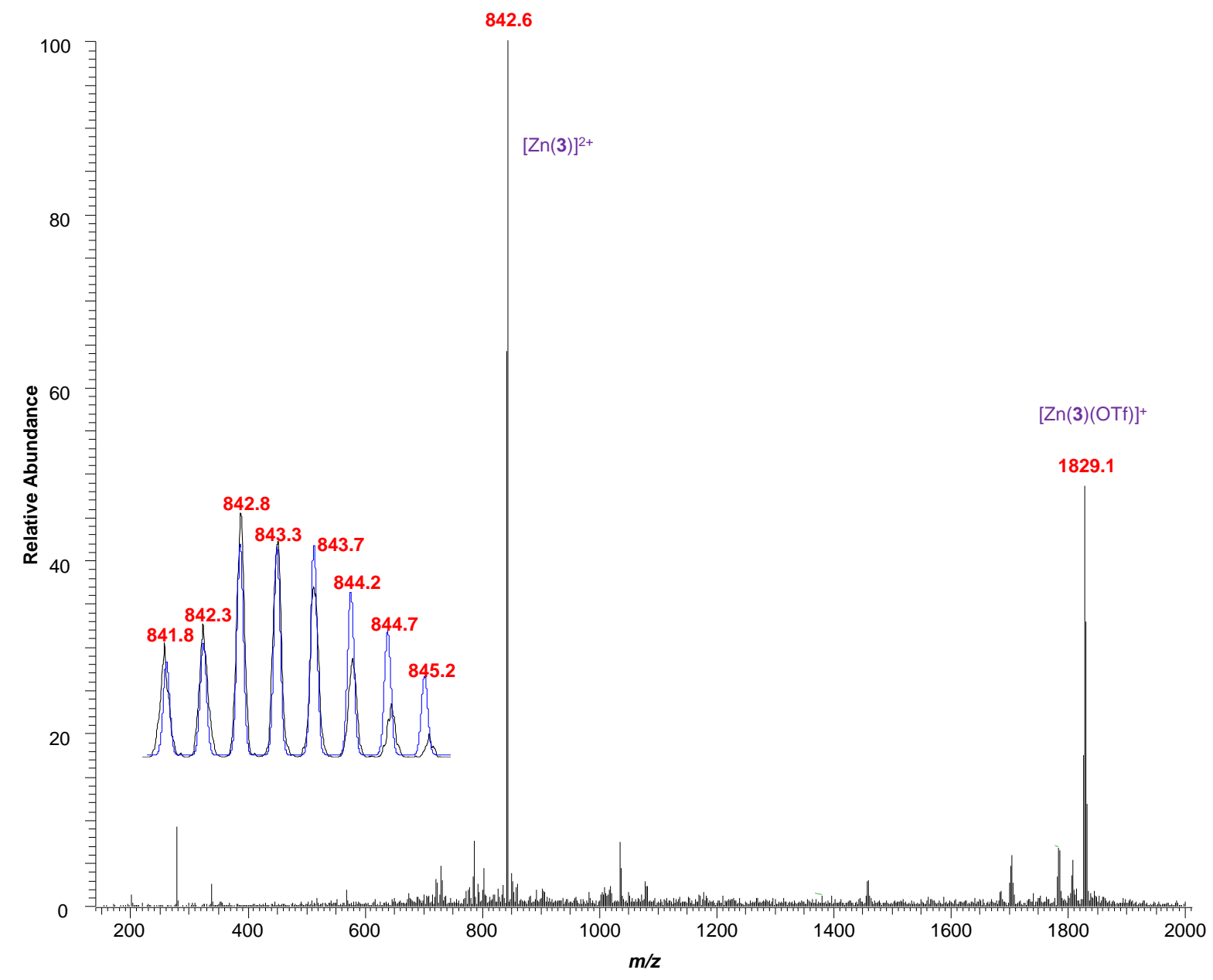

Figure S73: ESI-MS spectrum of complex $[\operatorname{Zn}(3)]^{2+}$. 


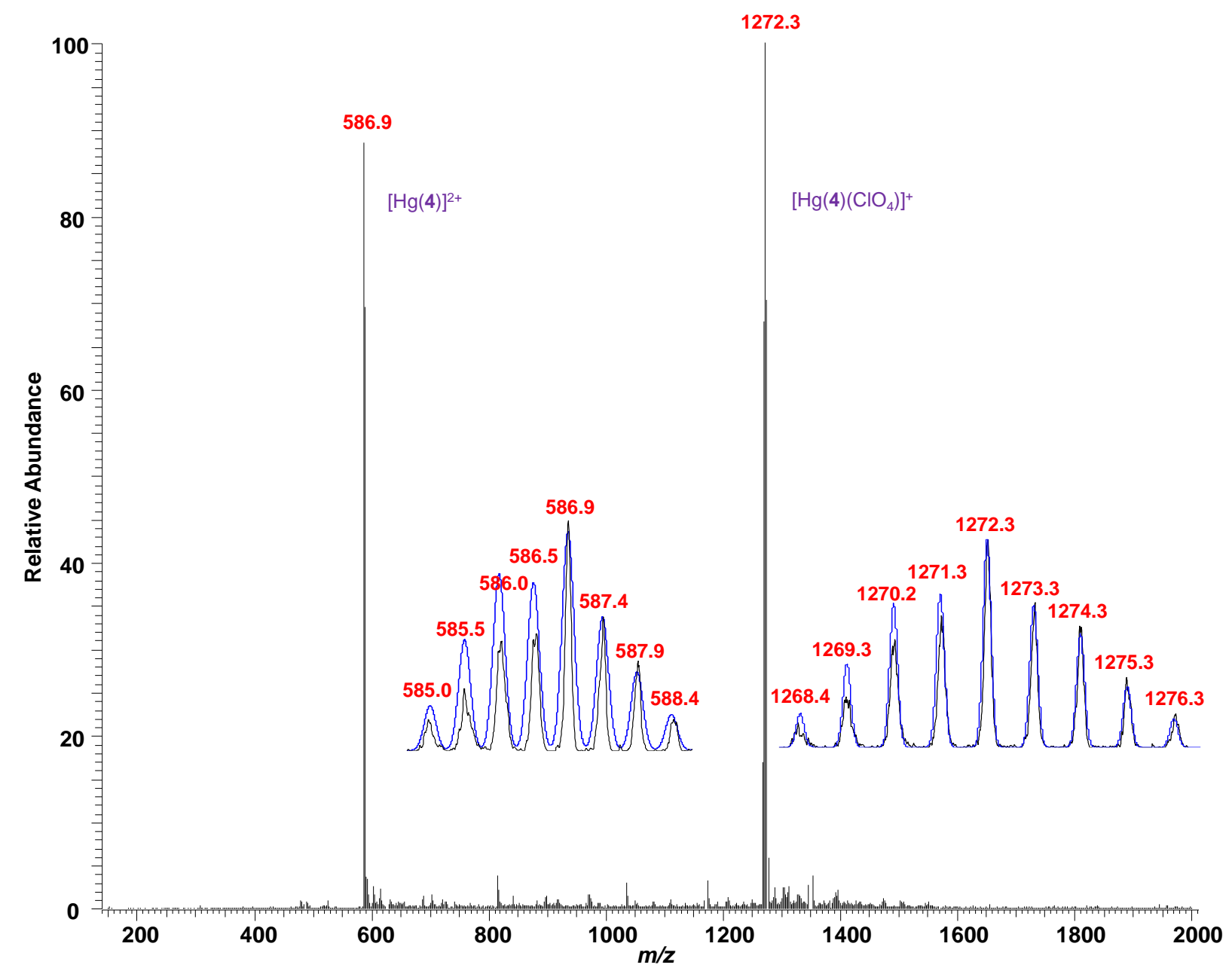

Figure S74: ESI-MS spectrum of complex $[\operatorname{Hg}(4)]^{2+}$. 


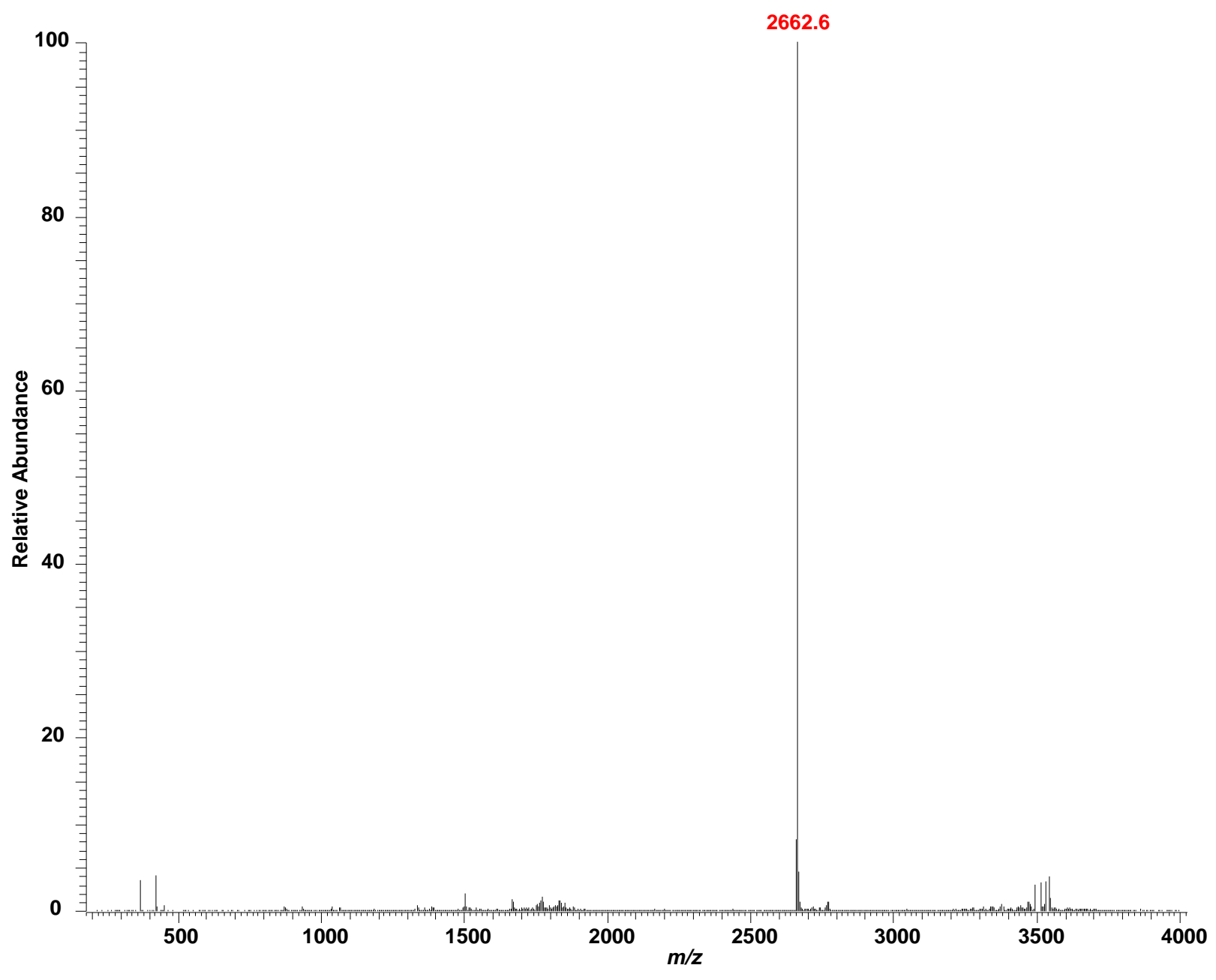

Figure S75: ESI-MS spectrum of ROT-1. 


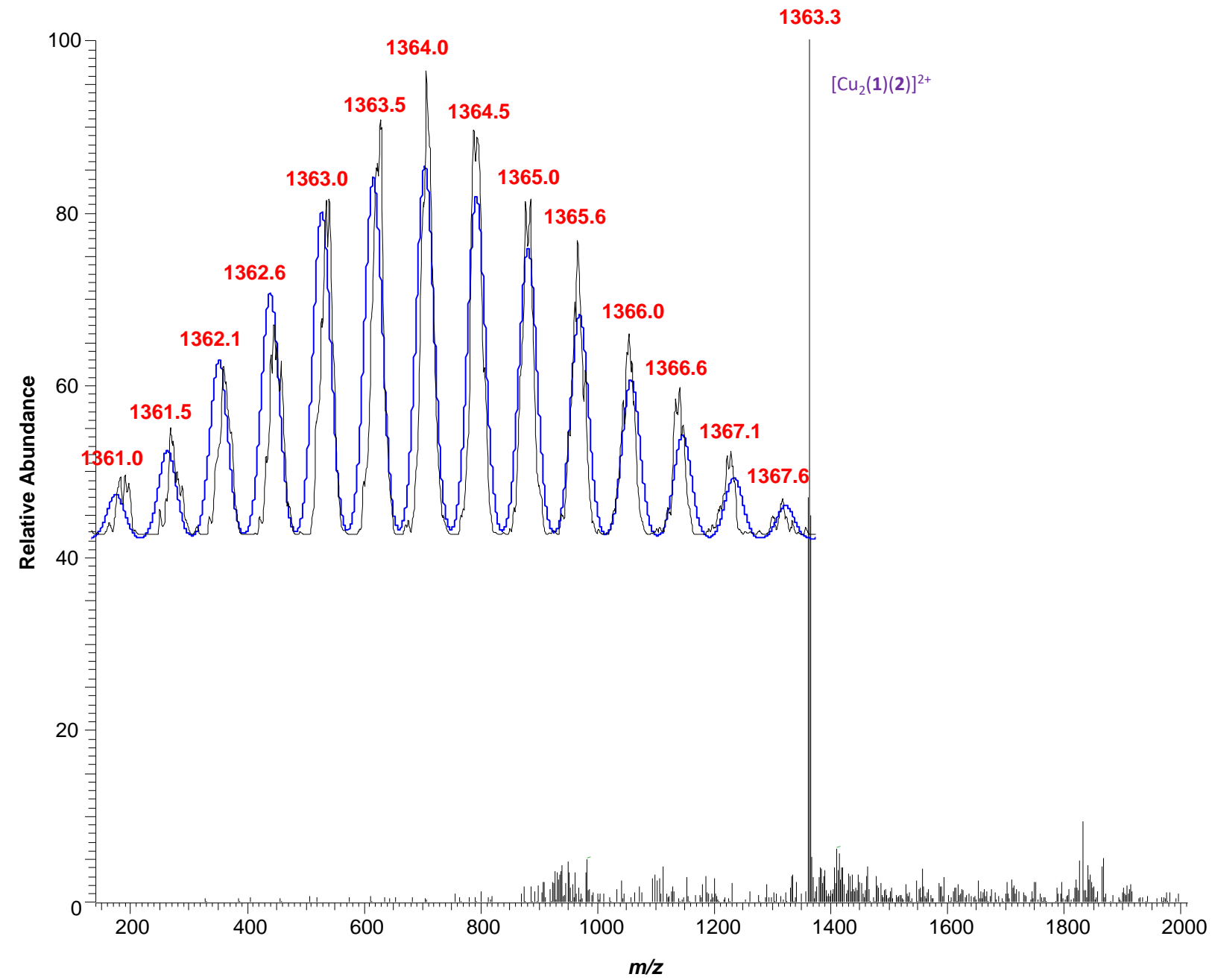

Figure S76: ESI-MS spectrum of ROT-2. 


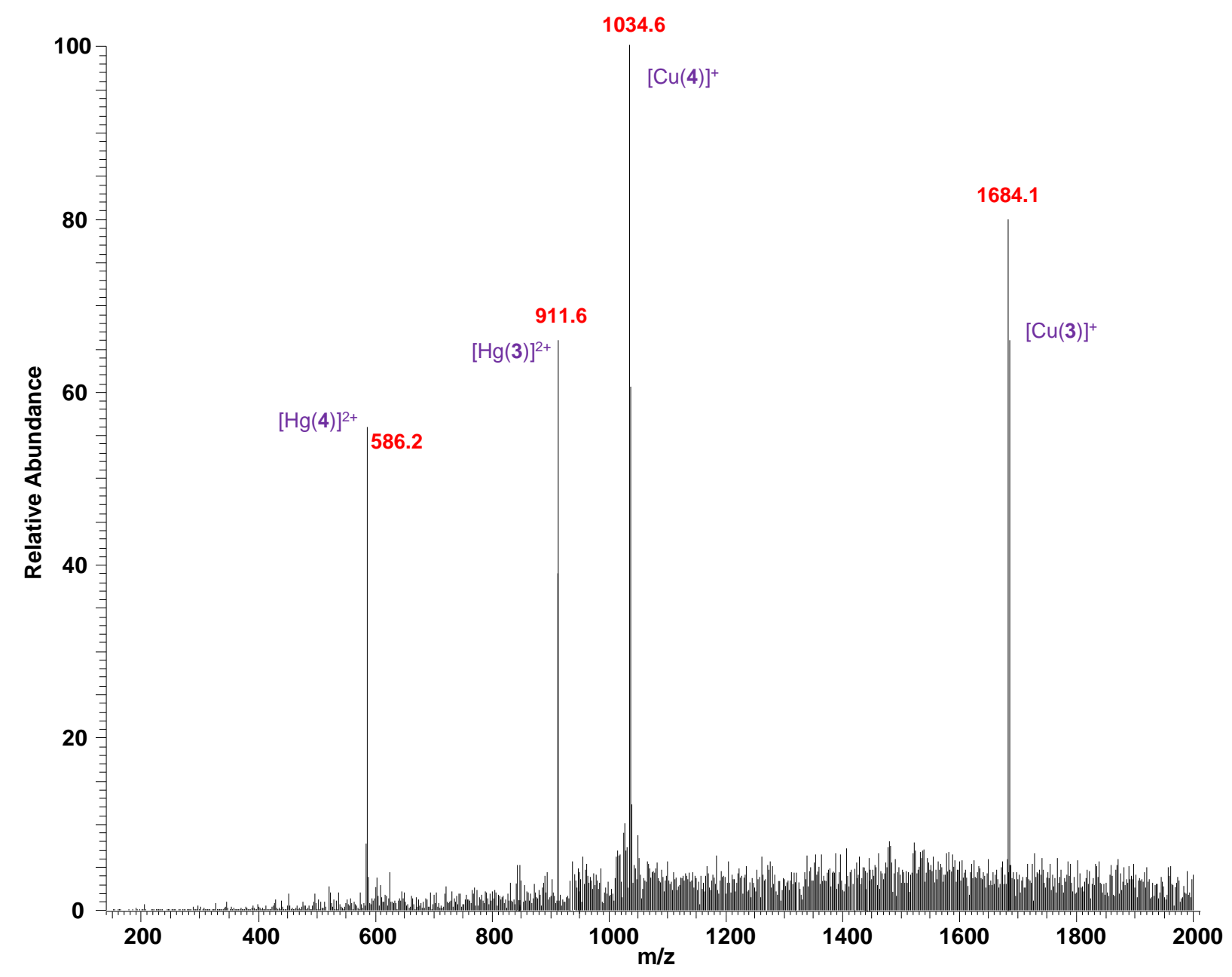

Figure S77: ESI-MS of state $(0,1)$ (without rotor assembly) showing the metal ion distribution on nanoswitches $\mathbf{3}$ and $\mathbf{4}$ when $\mathrm{Hg}^{2+}$ was added. The spectrum was measured immediately after addition of $\mathrm{Hg}^{2+}$. 


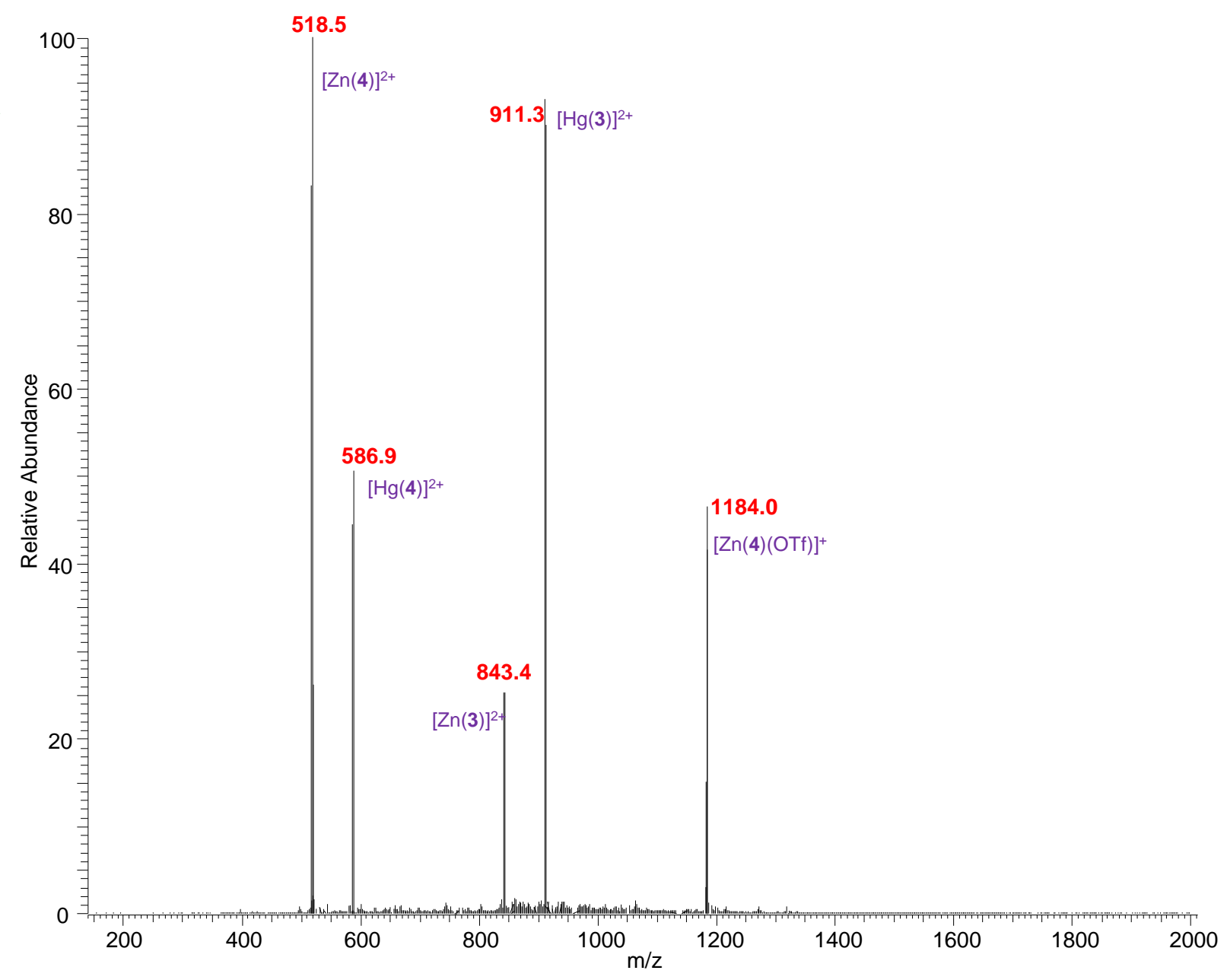

Figure S78: ESI-MS of state $(1,1)$ (without rotor assembly) showing the metal ion distribution on nanoswitches 3 and $\mathbf{4}$ after addition of $\mathrm{Hg}^{2+}$ and $\mathrm{Zn}^{2+}$. The spectrum was taken 30 min after addition of $\mathrm{Zn}^{2+}$ to state $(0,1)$. 


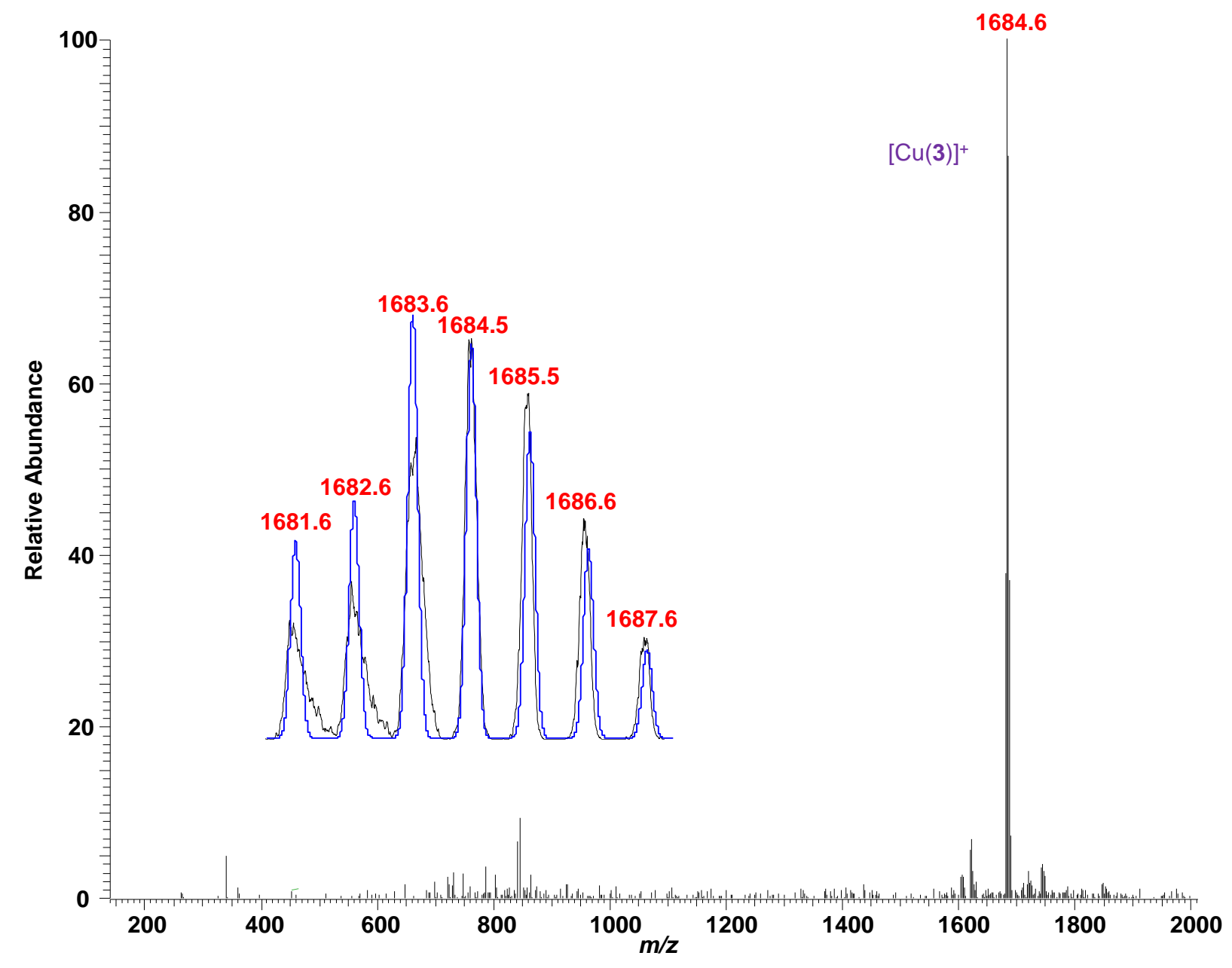

Figure S79: ESI-MS spectrum of state $(0,0)$. 


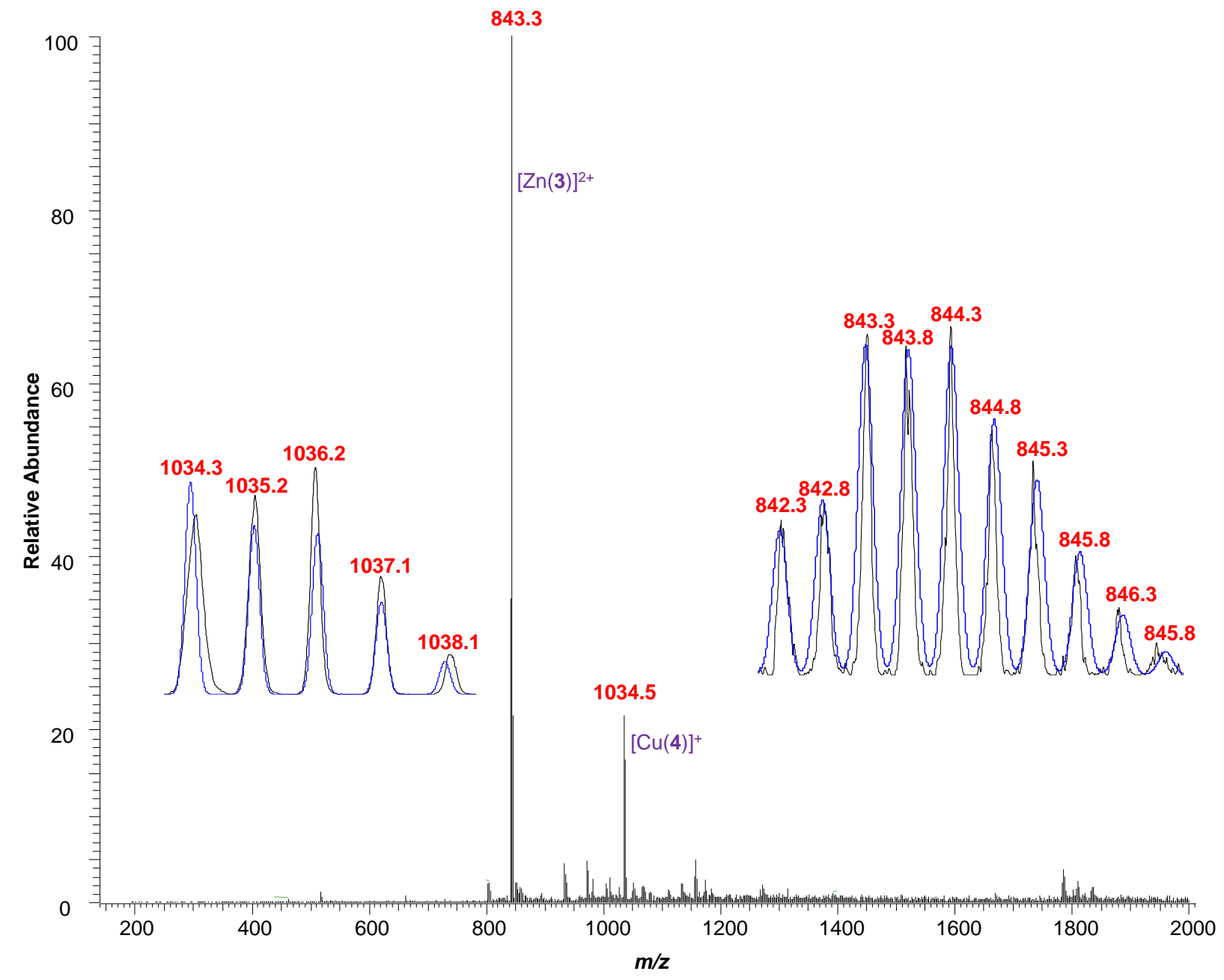

Figure S80: ESI-MS spectrum of state $(1,0)$. 


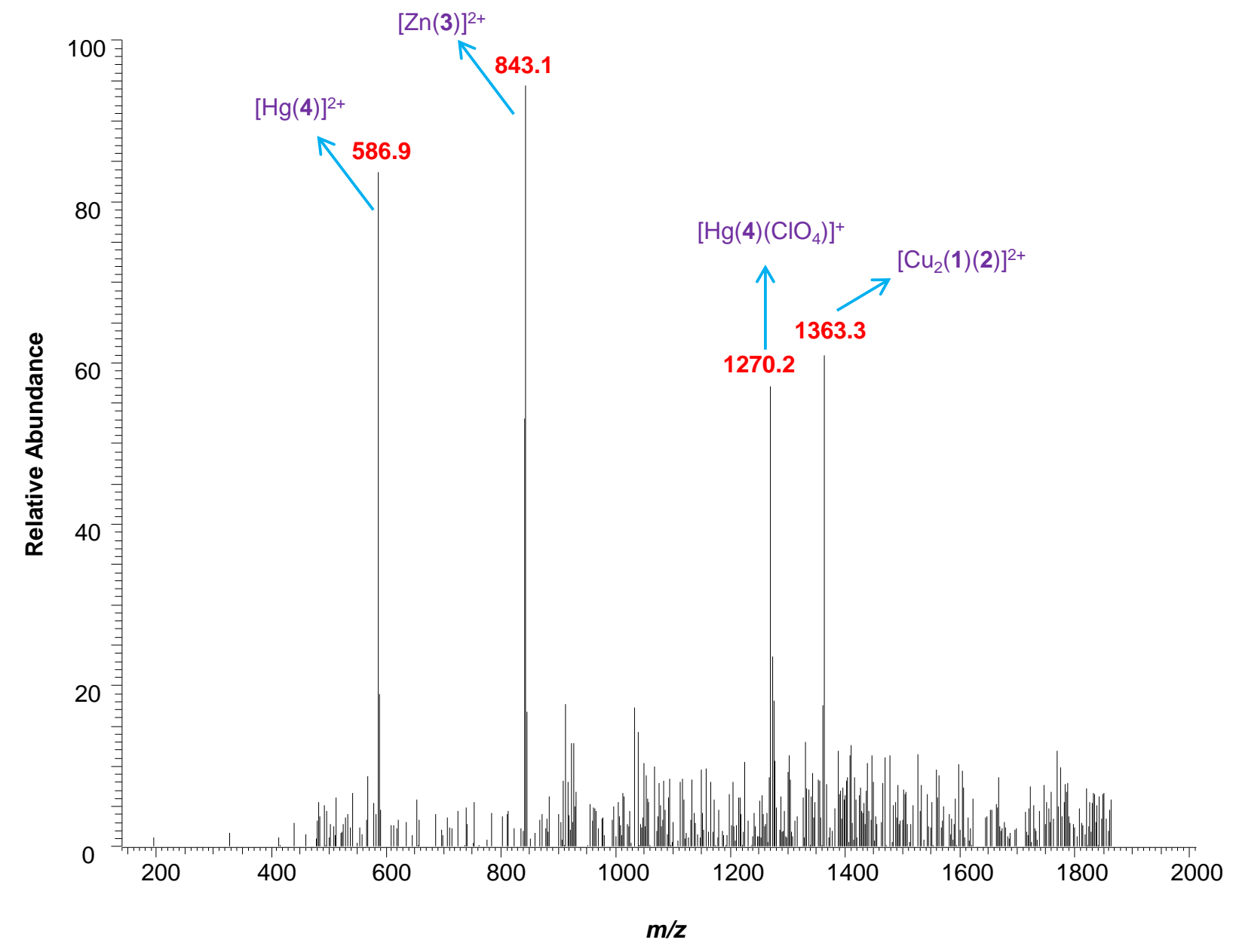

Figure S81: ESI-MS spectrum of state $(1,1)$. 


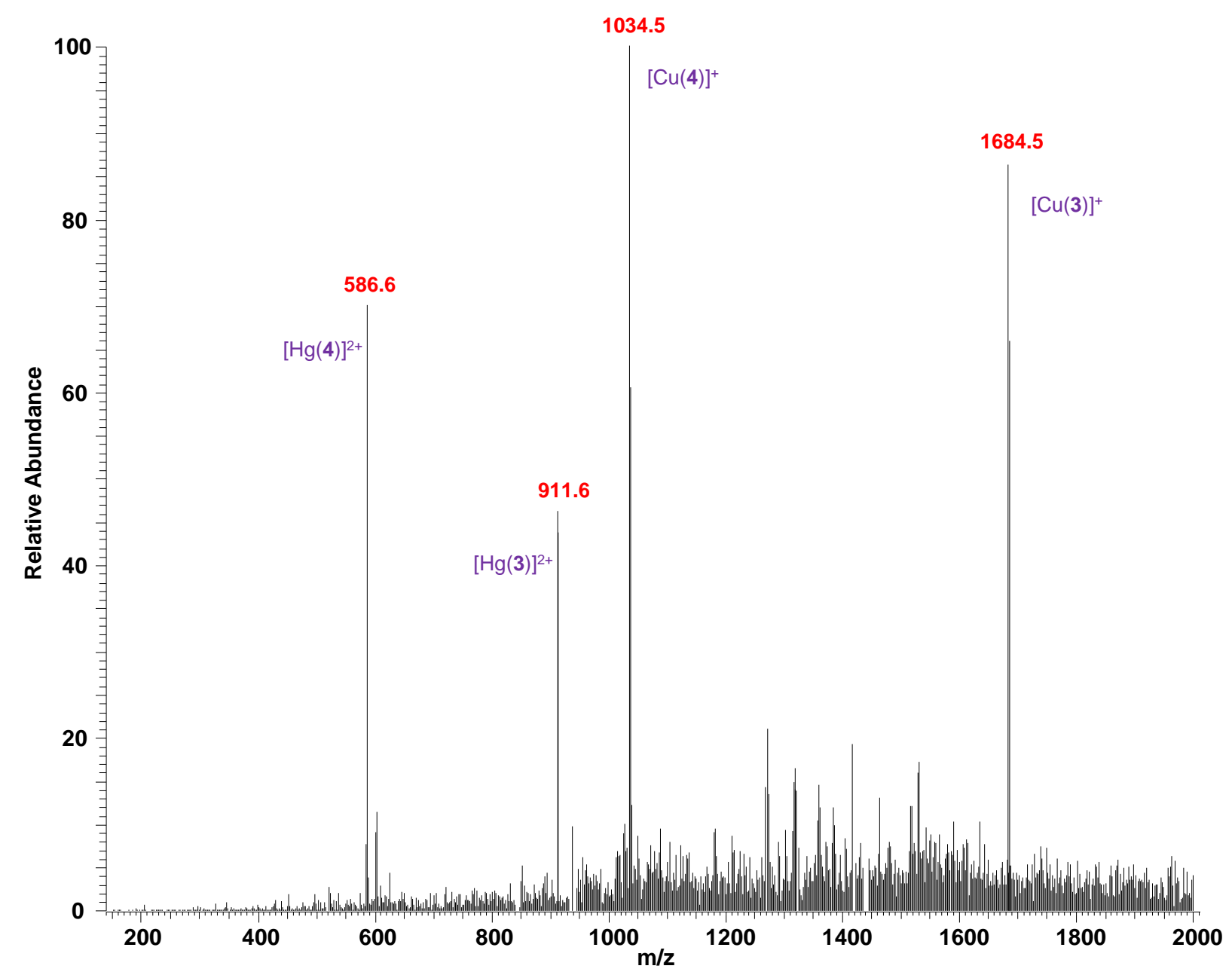

Figure S82: ESI-MS of $(0,1)$ state when $\mathrm{Hg}^{2+}$ is added first. 


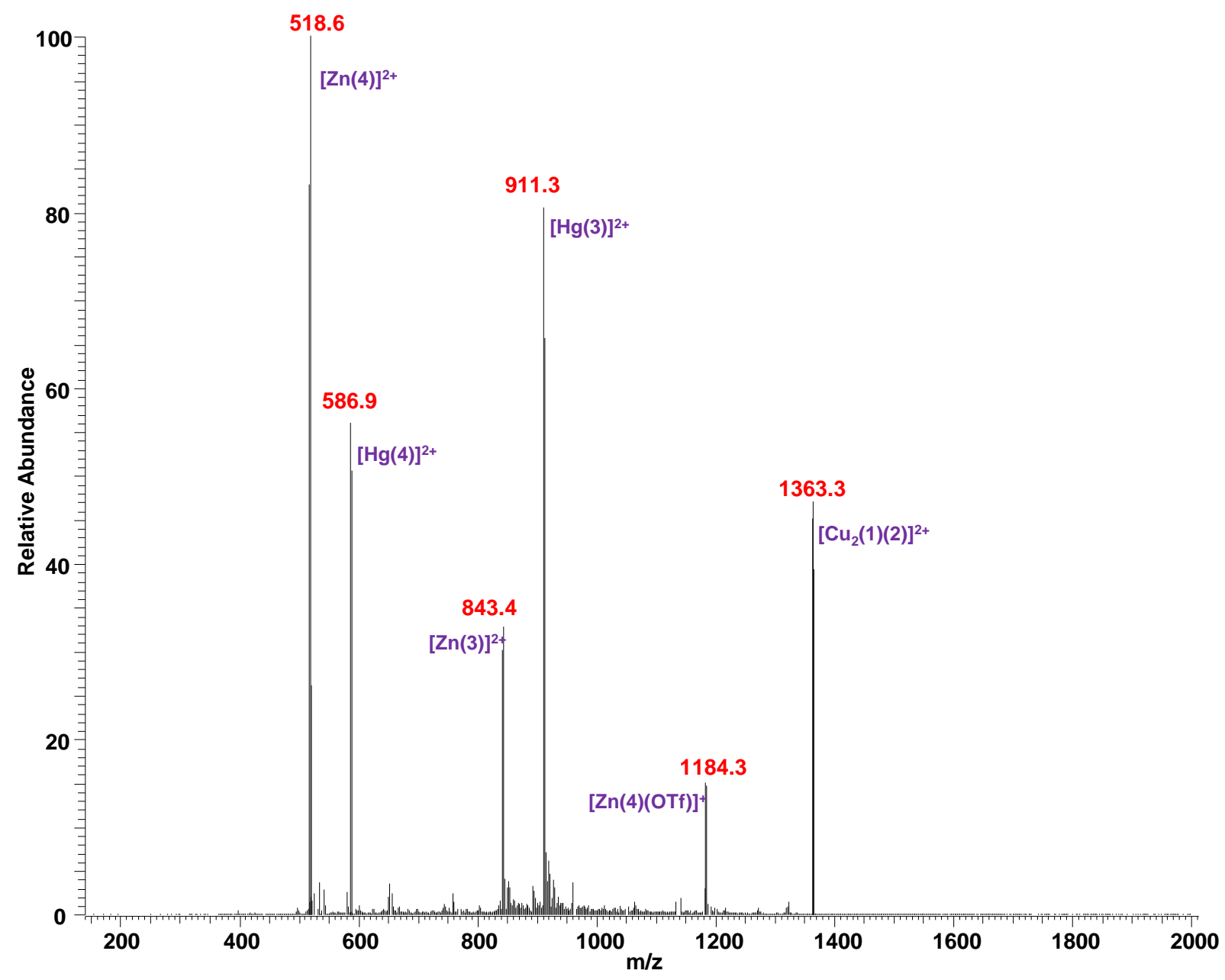

Figure S83: ESI-MS of state $(1,1)$ when $\mathrm{Hg}^{2+}$ is used as IN-1 and $\mathrm{Zn}^{2+}$ as $\mathbf{I N - 2}$. 


\section{Speciation Distribution Analysis}

With the binding constant between $\mathrm{Cu}^{+}$and 2,9-dimesityl-1,10-phenanthroline at hand $(\log K=$ 5.1) we analyzed the formation of the corresponding complex using the Hyperquad software (http://www.hyperquad.co.uk/hyss.htm). A refers to $\mathrm{Cu}^{+}$from $\left[\mathrm{Cu}\left(\mathrm{CH}_{3} \mathrm{CN}\right)_{4}\right] \mathrm{PF}_{6}$ and $\mathbf{B}$ corresponds to 2,9-dimesityl-1,10-phenanthroline. AB represents the complex $[\mathrm{Cu}(2,9-$ dimesityl-1,10phenanthroline) $]^{+}$.

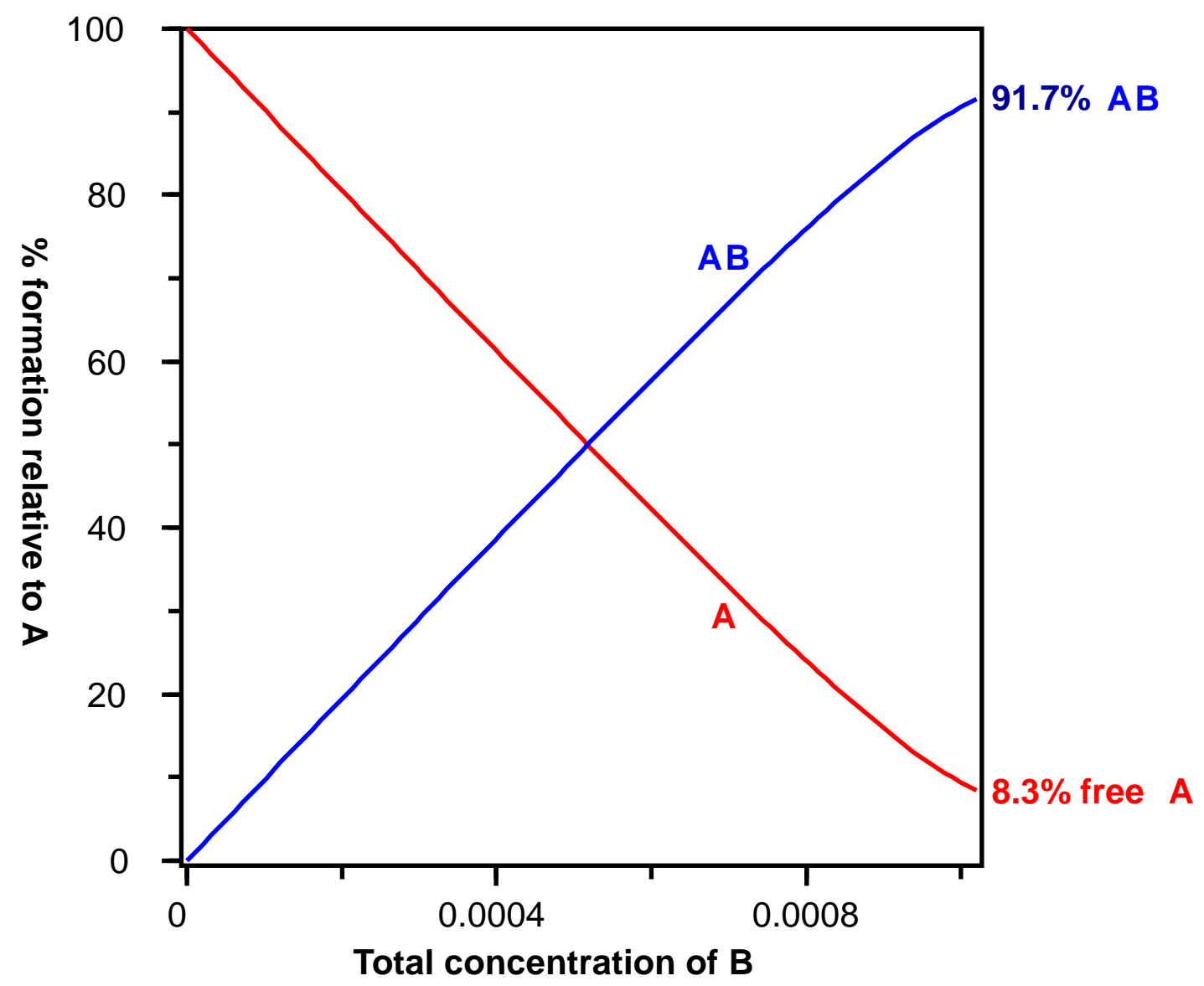

Figure S84: Speciation distribution of free and complexed $\mathrm{Cu}^{+}$(distribution was calculated for $1.02 \times 10^{-3} \mathrm{M} \mathrm{A}=\mathrm{Cu}^{+}$). 


\section{References}

(1) Alonso-Marañón, L.; Sarandeses, L. A.; Martínez, M. M.; Sestelo, J. P. Synthesis of fused chromenes by the indium(iii)-catalyzed cascade hydroarylation/cycloisomerization reactions of polyyne-type aryl propargyl ethers. Org. Chem. Front. 2018, 5, 2308-2312.

(2) Hu, Y.; Zhao, Z.; Bai, X.; Yuan, X.; Zhang, X.; Masuda, T. Organoborane-containing polyacetylene derivatives: synthesis, characterization, and fluoride-sensing properties. RSC $A d v$. 2014, 4, 55179-55186.

(3) Yang, X.; Lu, H.; Zhu, X.; Zhou, L.; Deng, G.; Yang, Y.; Liang, Y. Palladium-Catalyzed Cascade Cyclization of Alkene-Tethered Aryl Halides with o-Bromobenzoic Acids: Access to Diverse Fused Indolo[2,1-a]isoquinolines. Org. Lett. 2019, 21, 7284-7288.

(4) Gaikwad, S.; Schmittel, M. Five-State Rotary Nanoswitch. J. Org. Chem. 2017, 82, 343-352.

(5) Topchiy, M. A.; Ageshina, A. A.; Gribanov, P. S.; Masoud, S. M.; Akmalov, T. R.; Nefedov, S. E.; Osipov, S. N.; Nechaev, M. S.; Asachenko, A. F. Azide-Alkyne Cycloaddition (CuAAC) in Alkane SolventsCatalyzed by Fluorinated NHC Copper(I) Complex. Eur. J. Org. Chem. 2019, 1016-1020.

(6) Biswas, P. K.; Saha, S.; Paululat, T. Schmittel, M. Rotating Catalysts Are Superior Suppressing Product Inhibition by Anchimeric Assistance in Four-Component Catalytic Machinery. J. Am. Chem. Soc. 2018, 140, 9038-9041.

(7) Reich, H. J. NMR Spectrum Calculations: WinDNMR, Version 7.1.13. Department of Chemistry, University of Wisconsin.

(8) Eyring, H. The Activated Complex in Chemical Reactions. J. Chem. Phys. 1935, 3, 107-115. 